


\title{
METABOLIC FATE OF JASMONATES
}

\section{Dissertation}

\author{
for the award of the degree \\ "Doctor rerum naturalium" \\ of the University of Goettingen \\ within the doctorial program \\ "Plant Responses To Eliminate Critical Threats" \\ of the Georg-August University School of Science (GAUSS) \\ submitted by

\section{Sven Haroth} \\ from Alfeld (Leine), Germany
}

September 2018

Department of Plant Biochemistry

Albrecht-von-Haller-Institute for Plant Sciences

Georg-August University Goettingen 
Thesis committee

Prof. Dr. Ivo Feussner, Department of Plant Biochemistry, Albrecht-von-Haller-Institute for Plant Science, University of Goettingen

Prof. Dr. Christiane Gatz, Department of Plant Molecular Biology and Physiology, Albrecht-vonHaller-Institute for Plant Science, University of Goettingen

Prof. Dr. Ralf Ficner, Department of Structural Biology, Institute for Microbiology and Genetics, University of Goettingen

Members of the Examination Board

Referee: Prof. Dr. Ivo Feussner, Department of Plant Biochemistry, Albrecht-von-Haller-Institute for Plant Science, University of Goettingen

$2^{\text {nd }}$ Referee: Prof. Dr. Christiane Gatz, Department of Plant Molecular Biology and Physiology, Albrecht-von-Haller-Institute for Plant Science, University of Goettingen

Further members of the Examination Board

Prof. Dr. Ralf Ficner, Department of Structural Biology, Institute for Microbiology and Genetics, University of Goettingen

Prof. Dr. Andrea Polle, Department for Forest Botany and Tree Physiology, Buesgen-Institute, University of Goettingen

Prof. Dr. Volker Lipka, Department of Plant Cell Biology, Albrecht-von-Haller Institute for Plant Sciences, University of Goettingen

Prof. Dr. Petr Karlovsky, Molecular Phytopathology and Mycotoxin Research, University of Goettingen

Date of oral examination: $16^{\text {th }}$ October 2018 


\section{TABLE OF CONTENTS}

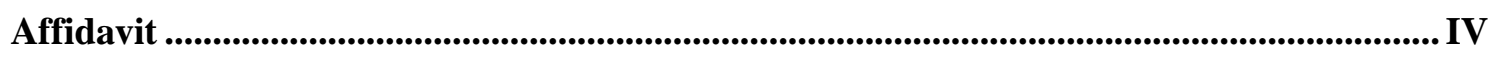

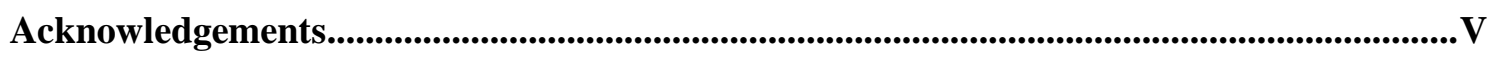

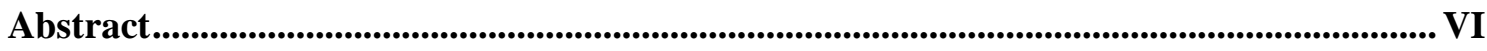

Abbreviations .................................................................................................................

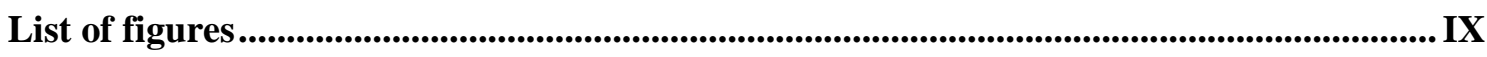

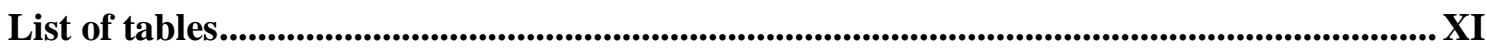

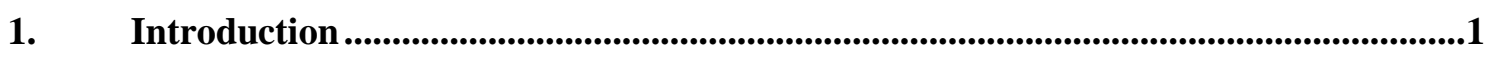

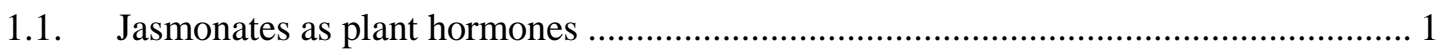

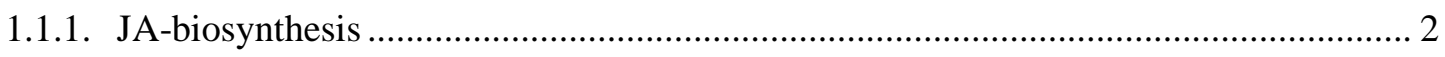

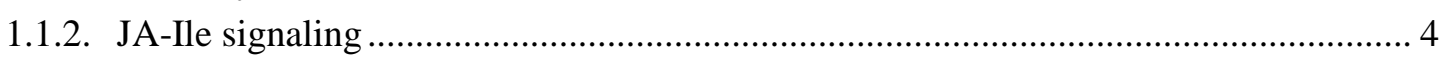

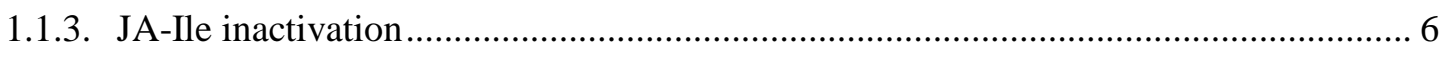

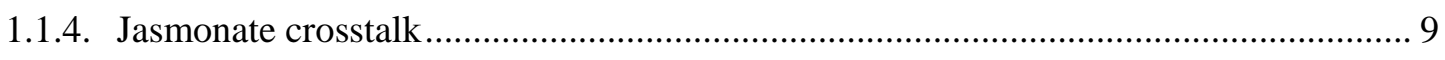

1.2. Glycosyltransferases in phytohormone homeostasis............................................ 10

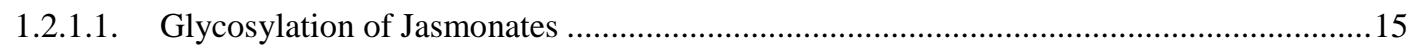

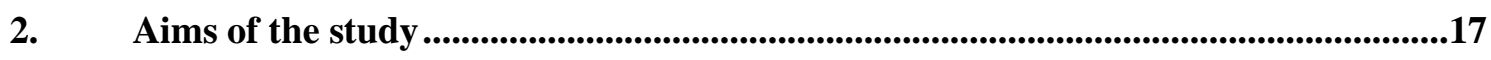

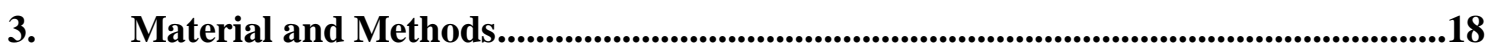

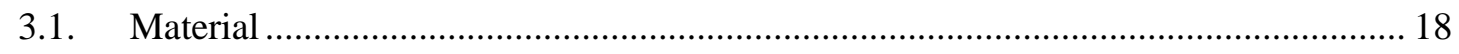

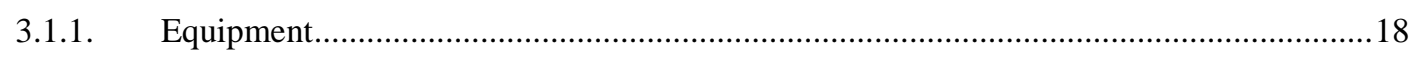

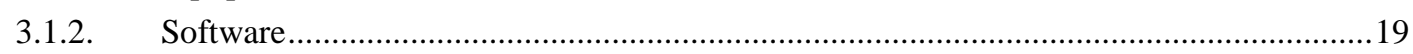

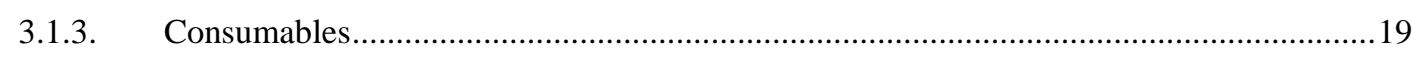

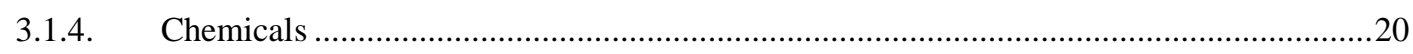

3.1.5. Enzymes, size markers, antibodies, and molecular biological kits .................................21

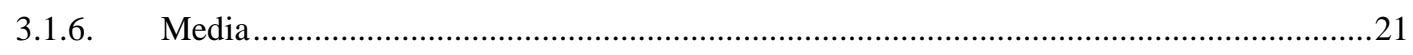

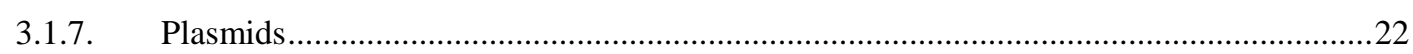

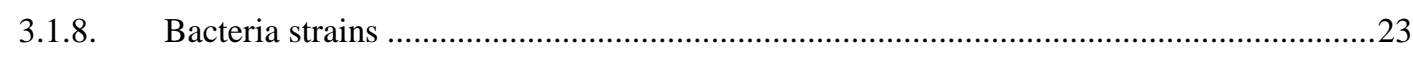

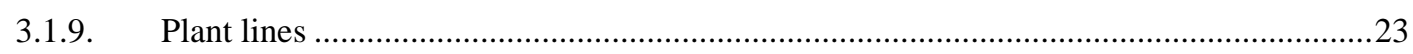

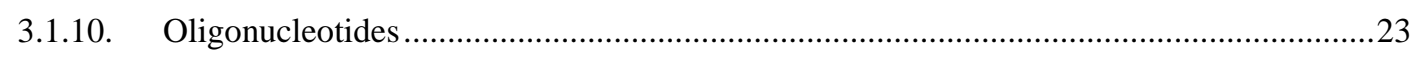

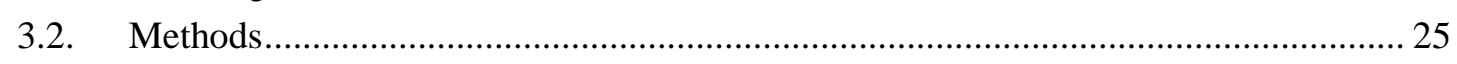

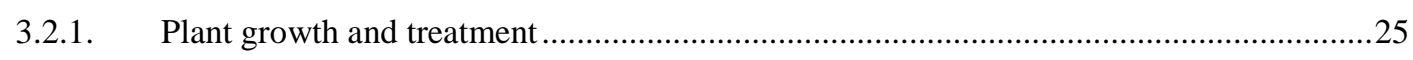

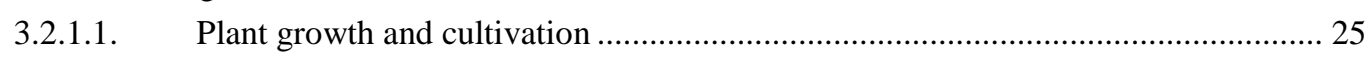

3.2.1.2. Seed propagation from JA-deficient plants by JA methyl ester treatment ............... 26

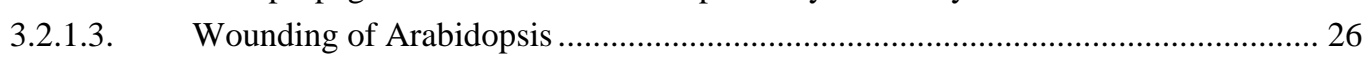

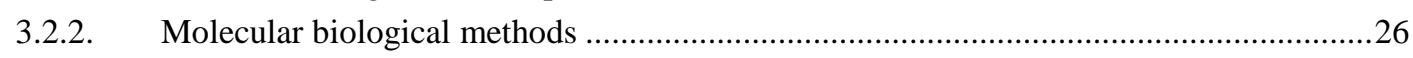

3.2.2.1. Extraction of genomic plant DNA ………................................................. 26

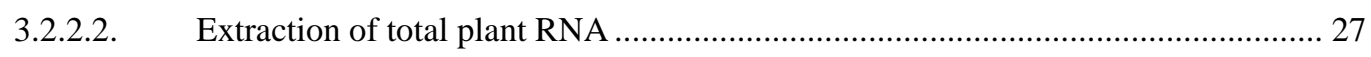

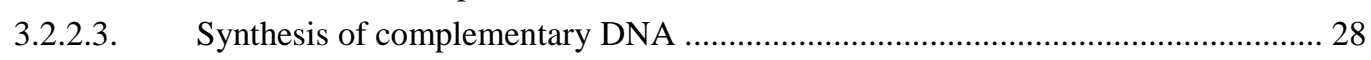

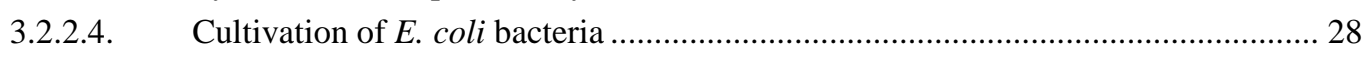

3.2.3. Cloning of coding sequences from A. thaliana into recombinant plasmids.......................29

3.2.3.1. Amplification of DNA fragments using PCR .................................................... 29

3.2.3.2. Separation of nucleotides by electrophoresis ...................................................... 29

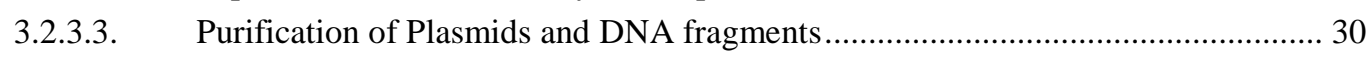

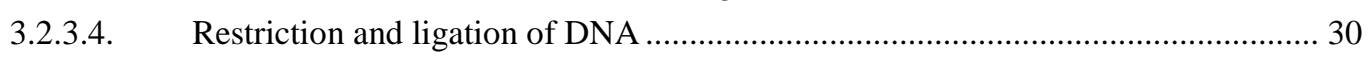

3.2.3.5. Transformation of competent E. coli cells ......................................................... 31 


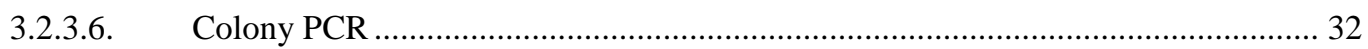

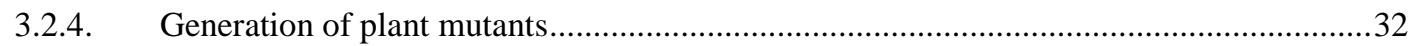

3.2.4.1. Gene editing by clustered regularly interspaced short palindromic repeats / CRISPR

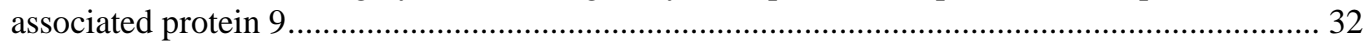

3.2.4.2. Transformation of Agrobacterium tumefaciens ................................................. 33

3.2.4.3. Agrobacterium-mediated transformation of A. thaliana ....................................... 33

3.2.4.4. Quantitative real-time PCR expression analysis .................................................. 34

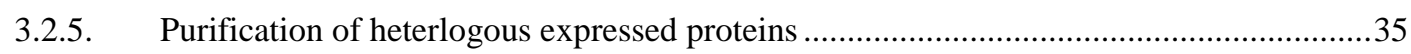

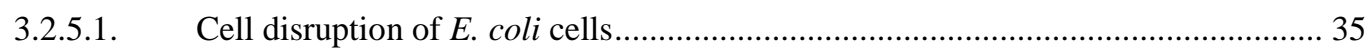

3.2.5.2. Recombinant protein purification ................................................................... 35

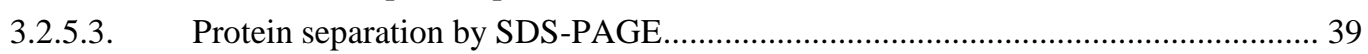

3.2.5.4. Protein detection by western blot ..................................................................... 40

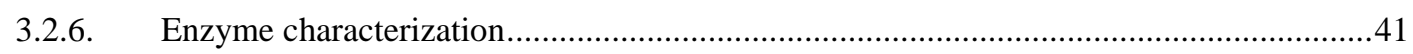

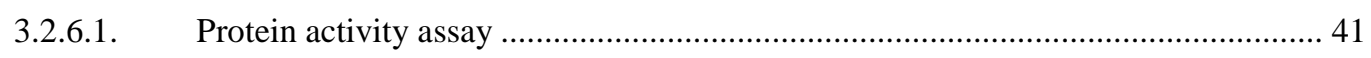

3.2.6.2. Determination of optimal temperature and $\mathrm{pH}$ values ........................................ 41

3.2.6.3. Analysis of the Substrate specificity of UGTs by using UDP- ${ }^{14} \mathrm{C}(\mathrm{U})-\mathrm{Glc} \ldots \ldots \ldots \ldots . . . .42$

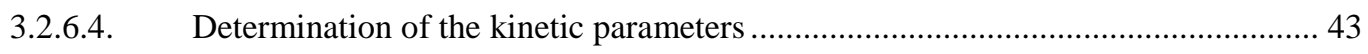

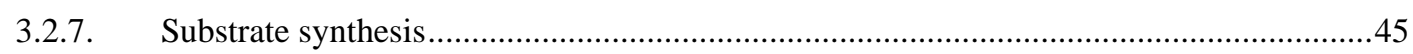

3.2.7.1. Enzymatic syntheses of 9- and 13-hydroxy-octadecatrienoic acids ...................... 45

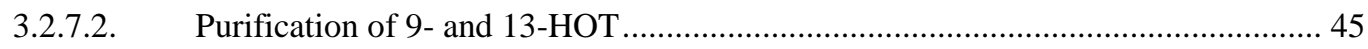

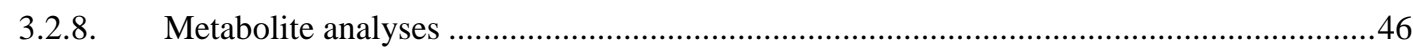

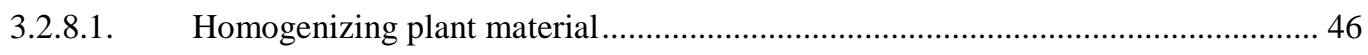

3.2.8.2. Extraction of plant material ............................................................................. 46

3.2.8.3. Targeted LC-MS/MS analysis of phytohormones ............................................ 47

3.2.8.4. Metabolite analysis by LC-high resolution-MS ................................................ 48

3.2.8.5. Non-targeted ex vivo metabolite fingerprinting ..................................................... 49

3.2.8.6. Structure determination by fragmentation .................................................. 50

4. Results ...........................................................................................................51

4.1. Identification of four UGTs as candidates for 12-OH-JA glycosylation ................... 51

4.1.1. UGT76E1 and UGT76E2 may be related to 12-OH-JA metabolism ...............................51

4.1.2. Transcriptome analysis of wounded A. thaliana leaves confirmed UGT76E1, UGT76E2 and identified $U G T 76 E 11$ and $U G T 76 E 12$ as wound induced transcripts ..................................53

4.1.3. Real time PCR analyses confirm, that UGT76E1, UGT7E11, and UGT76E12 are induced

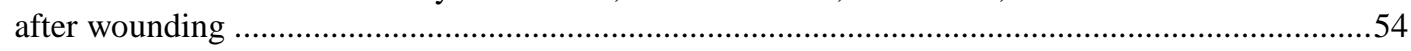

4.1.4. UGT76E1 and UGT76E2 are assigned to JA metabolism by co-expression analyses......55

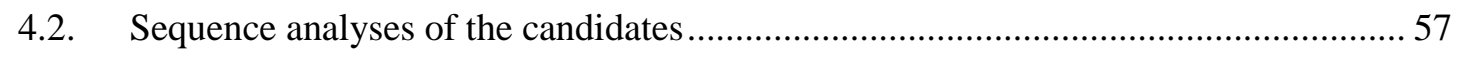

4.2.1. UGT76E1, UGT76E2, UGT76E11, and UGT76E12 have similar gene structures..........57

4.2.2. UGT76E1 and UGT76E2 as well as UGT76E11 and UGT76E12 cluster as

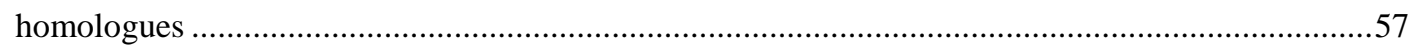

4.2.3. Protein parameters of UGT76E1, UGT76E2, UGT76E11, and UGT76E12 are similar ..59

4.3. Biochemical characterization of UGT76E1, UGT76E2, UGT76E11, and UGT76E12..

4.3.1. UGT76E1, UGT76E2, UGT76E11, UGT76E12, and UGT74F1 were heterologously expressed in E. coli .

4.3.2. UGT76E1, UGT76E2, UGT76E11, UGT76E12, and UGT74F1 were purified to homogeneity.

4.3.3 UGT76E1, UGT76E2, UGT76E11, UGT76E12, and UGT74F1 show activity towards $\omega-$

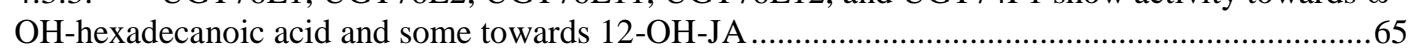

4.3.4. The UGT enzymes prefer mild alkaline $\mathrm{pH}$..............................................................68

4.3.5. UGT76E1 and UGT76E2 show substrate preference for 12-OH-JA...........................69 
4.3.6. Homogenous enzymes can be brought back to their native substrates

4.3.7. UGT76E1, UGT76E2, UGT76E12, and UGT74F1 show activity towards their known substrates

4.3.8. UGT76E1, UGT76E2, UGT76E11, UGT76E12, and UGT74F1 show activity towards native substrates

4.3.8.1. 12-OH-JA-Ile is no substrate for the analyzed UGTs ....................................... 78

4.3.9. Substrate preferences of UGT76E1, UGT76E2, UGT76E11, UGT76E12, and UGT74F1 .......

4.3.10. The kinetic Parameters of UGT76E1, UGT76E2, UGT76E11, and UGT76E12 were

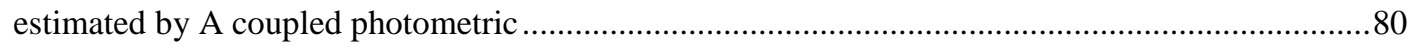

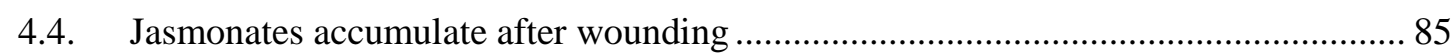

4.5. Gene editing enables investigating UGT76E1, UGT76E2, UGT76E11, and

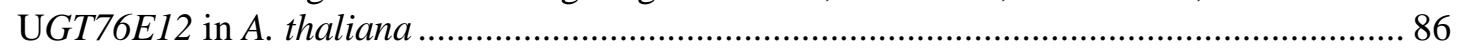

4.5.1. The optimized vector system targets four candidates to Cas9 .......................................87

4.5.2. CRISPR/Cas9 initiates a loss-of-function mutation in $U G T 76 E 1$................................88

5. Discussion ........................................................................................................90

5.1. Comprehensive characterization of the JA-related UGTs revealed their catalytic

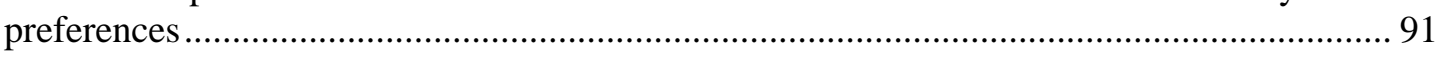

5.2. UGT76E1, UGT76E11, and UGT76E12 are slow catalysts .............................. 93

5.3. UGT76E1 glycosylates 12-OH-JA with high specificity .....................................96

5.4. UGT76E2 forms 12-O-Glc-JA with high specificity and turnover numbers ............. 98

5.5. UGT76E11 shows activities towards oxylipins and a so far unknown metabolite ..... 99

5.6. UGT76E12 prefers other oxylipins over 12-OH-JA ......................................... 100

5.7. Evidence for physiological relevance of 12-O-Glc-JA ......................................... 102

5.7.1. 12-O-Glc-JA-Ile cannot be identified in A. thaliana ...................................................... 104

5.7.2. 12-COOH-JA was identified in wounded leaves of $A$. thaliana ...................................... 104

6. Outlook ...................................................................................................................106

7. References .........................................................................................................108

8. Supplemental data..........................................................................................117

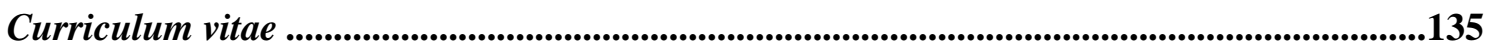




\section{AfFIDAVIT}

Hereby, I confirm that this dissertation entitled "Metabolic fate of jasmonates" has been written independently and no other sources and aids were used than quoted.

\section{Sven Haroth}

Goettingen September $3^{\text {rd }}, 2018$ 


\section{ACKNOWLEDGEMENTS}

The space here is far too little to thank everybody as I should but I will give it a try:

First of all, I thank Prof. Dr. Ivo Feussner for supervising me for a long time. He took care of my scientific education from Bachelors, over Masters, to this dissertation in his department of Plant Biochemistry. Thank you a lot for all your support and trust through many years! I am also indebted to Prof. Dr. Chrsitiane Gatz and Prof. Dr. Ralf Ficner as members of the thesis committee and for helpful and interesting discussions.

In addition, I am deeply grateful for the support of Dr. Kirstin Feussner. She answered many everyday questions, helped me with metabolite measurements, fragmentations, with her MarVisexperience, as well as with uncountable discussions, and not to forget for critical reading of this thesis. Kirstin, thank you so much for all your support and patience with me. Furthermore, I thank Dr. Ellen Hornung, she helped and supported me over a long time of my study. I cannot tell how much helpful advice and motivating words she had for me, thank you Ellen - also for many delicious lunch meals. Dr. Amélie Kelly was a great support with the gene editing method, and by critical reading parts of this thesis. Dr. Cornelia Herrfurth and Dr. Krzysztof Zienkiewicz supported me a lot with phytohormone measurements. I thank Dr. Stefanie König for initiating the work. Sabine Freitag not only produced and purified oxylipins for my work and helped me with liquid chromatography, but also supported me as a good friend over years. You cheered me up every day, thank you. Furthermore, I want to thank Susanne Mester for taking care of my plants. I thank Dr. Alexander Kaever and Manuel Landesfeind for providing the MarVis software as well as Prof. Dr. Sabine Rosahl (University of Halle/Wittenberg, Germany) for providing the pET3B vector with the potato lipoxygenase. This thesis was performed within the International Research Training Group 2172 "PRoTECT: Plant Responses To Eliminate Critical Threats", which gave me great scientific opportunities and many new friends.

I thank all members of the department of Plant Biochemistry, especially all PhD students, Dmitrij, Milena, Yi-Tse, Hanno, Katharina, Jasmin, Elisa, Athanas, and Franziska as well as former doctorial students Dr. Viktoria Bruckhoff, Dr. Daniel Bruckhoff, and Dr. Heike Siegler for the wonderful atmosphere, great barbeques and evenings - I enjoyed working here every day. My fellow students and friends, Sven Johansson, Sören Rindfleisch, and Florian Harmann, made studying a great time of my life, thank you so much! Next, I want to thank my mother Ute Haroth and my grandmother Inge Bensch for endless understanding and support during the last years and forever. Nothing would have been possible without you. Last but not least, I want to thank my girlfriend Marlene Jost! Especially, the last weeks were hard for both of us, thank you for all your love. On to the next adventure... 


\section{AbSTraCT}

Jasmonoyl-isoleucine (JA-Ile) is a phytohormone that orchestrates responses to wounding, feeding insects or necrotrophic pathogens in plants. JA-Ile-metabolism has been studied intensively (Wasternack, 2015) and most of the enzymes of the JA-Ile-synthesis as well as of its catabolism have been described (Koo, 2018; Wasternack, 2015). However, the enzyme(s) responsible to specifically glycosylate 12-hydroxy-JA (12-OH-JA) are still elusive although the metabolite 12-O-glucosyl-JA (12-O-Glc-JA) has been described in plants (Koo, 2018; Miersch et alia (et al.), 2008; Seto et al., 2009). 12-O-Glc-JA triggers tuber formation in potato (Yoshihara et al., 2014) and induces leaf-closing in Mimosa (Nakamura et al., 2011). Furthermore, the glycoside might have individual functions as storage- or transport-compound (Miersch et al., 2008).

Therefore, this work investigated the glycosylation of 12-OH-JA. Four UDP-dependent glycosyltransfereases (UGTs) (UGT76E1, UGT76E2, UGT76E11, and UGT76E12) were identified as wound-induced and JA-related and sequence-analyses identified the catalytic motifs in all of them. UGT76E1, UGT76E2, UGT76E11, and UGT76E12 were heterologously expressed and purified from bacterial cultures. The UGT enzymes were characterized biochemically by different activity assays in conditions as equal as possible to determine their individual substrate tolerances and specificities. In addition, a new approach was established which exposes the enzymes to their native substrate to challenge their selectivity. Through this approach, it was possible to investigate purified proteins as close as possible to their natural environment. Finally, the kinetic parameters of the UGTs were recorded with their preferred substrates in a continuous assay. Altogether, UGT76E1 and UGT76E2 were characterized as 12-OH-JA-UGTs. UGT76E11 showed activity towards oxylipins and an unknown compound with the calculated sum formula C11H18O3. UGT76E12 showed high activity towards an artificial hydroxy-FA, and three oxylipins as well as to 12-OH-JA with minor extent.

This is the first report of UGTs that specifically glycosylate oxylipins. So far, glycosylated oxylipins have been found in L. paucicostata (Kai et al., 2010) but not in A. thaliana. However, glycosylation may increase the solubility of the FA-derived compounds in the plant cell and may be involved in oxylipin regulation or even signaling (confer Wasternack \& Feussner, 2018).

To investigate the physiological role of the candidate UGTs, a CRISPR/Cas9 approach was designed. A ugt765e1 mutant plant line was originally identified by screening for an altered metabolite profile of $12-\mathrm{OH}-\mathrm{JA}, 12-\mathrm{OH}-\mathrm{JA}$ sulfate, and $12-\mathrm{O}$-Glc-JA. Due to potential redundancy of the candidates forming $12-O$-Glc-JA, higher order mutants will be necessary to investigate the consequences of a $12-O$-Glc-JA-depletion in vivo. 


\section{Abbreviations}

\begin{tabular}{|c|c|c|c|}
\hline$[\mathrm{E}]$ & Concentration od enzyme & cps & Counts per second \\
\hline$[\mathrm{S}]$ & Concentration of substrate & CRISPR & Clustered regularly interspaced \\
\hline \multirow{2}{*}{$11-\mathrm{HHT}$} & $(7 Z, 9 E, 13 Z)-11-H y d r o x y-7,9,13-$ & & short palindromic repeats \\
\hline & hexadecatrienoic acid & CTAB & Cetyltrimethylammonium \\
\hline \multirow[t]{2}{*}{ 11-O-Glc-HHT } & 11-O-Glycosyl-hydroxy- & $\mathrm{d}$ & Days \\
\hline & hexadecatrienoic acid & dde2-2 & Delayed dehiscence 2-2 \\
\hline 12,13-EOT & 12,13-Epoxy-octadecatrienoic acid & ddh-JA & Didehydro-jasmonic acid \\
\hline 12-COOH-JA & 12-Carboxy-jasmonic acid & DEPC & Diethyl pyrocabonate \\
\hline 12-COOH-JA- & 12-Carboxy-jasmonoyl-isoleucine & DES & Divinyl ether synthase \\
\hline Ile & & dh-Kaempferol & Dihydro-kaempferol \\
\hline $12-\mathrm{HSO}_{4}-\mathrm{JA}$ & 12-Hydroxy-jasmonic acid sulfate & dh-Myricetin & Dihydro-myricetin \\
\hline 12-O-Glc-JA & 12-O-Glucosyl-jasmonic acid & dnOPDA & Dinor-12-oxo-phytodienoic acid \\
\hline \multirow{2}{*}{ 12-O-Glc-JA-Ile } & 12-O-Glucosyl-jasmonoyl- & dNTP & Deoxyribonucleoside triphosphates \\
\hline & isoleucine & DP & Declustering potential \\
\hline 12-OH-JA & 12-Hydroxy-jasmonic acid & DTT & Dithiothreitole \\
\hline 12-OH-JA-Ile & 12-Hydroxy-jasmonoyl-isoleucine & e. $g$ & Exempli gratia \\
\hline 12-OPDA & cis-(+)-12-Oxo-phytodienoic acid & EAS & Epoxy alcohol synthase \\
\hline \multirow[t]{2}{*}{ 13-HOT } & $(9 Z, 11 E, 15 Z)-13$-Hydroxy- & EDTA & Ethylenediaminetetraacetic acid \\
\hline & 9,11,15-octadecatrienoic acid & EIC & Extracted ion chromatogram \\
\hline \multirow[t]{2}{*}{ 13-HPOT } & $(9 Z, 11 E, 15 Z)-13$-Hydroperoxy- & $\mathrm{EP}$ & Entrance potential \\
\hline & $9,11,15$-octadecatrienoic acid & ESI & Electrospray ionization \\
\hline 13-LOX & 13-Linoleate lipoxygenase & et al. & Et alia \\
\hline${ }^{14} \mathrm{C}(\mathrm{U})-\mathrm{Glc}$ & Uniformly ${ }^{14} \mathrm{C}$ labelled glucose & EVC & Empty vector control \\
\hline $16: 0$ & Hecadecanoic acid & f. w. & Fresh weight \\
\hline \multirow[t]{2}{*}{ 1D-SOM } & One-dimensional self-organizing & FA & Fatty acid \\
\hline & map & GA & Gibberellic acid \\
\hline 2-O-Glc-SA & 2-O-Glucosyl-salicylic acid & GE & Glycosyl ester \\
\hline 2-OH-16:0 & 2-Hydroxy-hexadecanoic acid & GH3 & Gretchen Hagen 3 \\
\hline 3-OH-16:0 & 3-Hydroxy-hexadecanoic acid & Glc & Glucose \\
\hline \multirow[t]{2}{*}{ 9-HOT } & $(10 E, 12 Z, 15 Z)-9-H y d r o x y-$ & GmLOX & Lipoxygenase from Glycine $\max$ \\
\hline & 10,12,15-octadecatrienoic acid & gRNA & GuideRNA \\
\hline \multirow[t]{2}{*}{ 9-HPOT } & $(10 E, 12 Z, 15 Z)-9$-hydroxy- & GTR1 & Glucosinolate transporter 1 \\
\hline & 10,12,15-octadecatrienoic acid & $\mathrm{h}$ & Hours \\
\hline 9-LOX & 9-Linoleate lipoxygenase & HisTag & Hexa-histidine tag \\
\hline ABA & Abscisic acid & HMPA-Glc & $(2 R, 3 R)-2$-Hydroxy-3- \\
\hline $\mathrm{ACN}$ & Acetonitrile & & methylpentanoic acid glycoside \\
\hline $\mathrm{AOC}$ & Allene oxide cyclase & HPL & Hydroperoxide lyase \\
\hline AOS & Allene oxide synthase & HPLC & High performance liquid \\
\hline $\mathrm{AP}$ & Alkaline phosphatase & & chromatography \\
\hline appr. & approximately & hpw & Hours post wounding \\
\hline $\mathrm{AU}$ & Absorption unit & IAA & Indole-3-acetic acid \\
\hline BA & Benzoic acid & IAR3 & Indole-3-acetic acid-alanine \\
\hline bp & Base pairs & & resistant 3 \\
\hline BRB & Britton-Robinson buffer & IBA & Indole-3-butyric acid \\
\hline BSA & Bovine serum albumin & ICA & Indole-3-carboxylic acid \\
\hline c & Concentration & ILL6 & Indole-3-acetic acid-leucine-like 6 \\
\hline \multirow[t]{2}{*}{ Cas9 } & $\begin{array}{l}\text { Clustered regularly interspaced } \\
\text { short palindromic repeats }\end{array}$ & IMAC & $\begin{array}{l}\text { Immobilized metal affinity } \\
\text { chromatography }\end{array}$ \\
\hline & associated protein 9 & JA & Jasmonic acid \\
\hline CAZy & Carbohydrate-active enzyme & JA-GE & Jasmonic acid-glucosyl ester \\
\hline cDNA & Complementary DNA & JA-Ile & Jasmonoyl-isoleucine \\
\hline $\mathrm{CE}$ & Collision energy & JA-Ile/Leu & Jasmonoyl-isoleucine/leucine \\
\hline$c f$. & Confer & JA-Leu & Jasmonoyl-leucine \\
\hline COI1 & Coronatine insensitive 1 & JA-ME & Jasmonic acid-methyl ester \\
\hline Col-0 & Columbia 0 & JAR1 & Jasmonic acid resistance 1 \\
\hline
\end{tabular}




\begin{tabular}{|c|c|c|c|}
\hline JAT1 & Jasmonic acid transporter 1 & Q-TOF & \\
\hline \multirow[t]{2}{*}{ JAZ } & Jasmonate inflorescence meristem & Red & Reductase \\
\hline & domain protein & $\mathrm{R}-O-\mathrm{Glc}$ & $O$-Glucosyl-substrate \\
\hline \multirow[t]{2}{*}{ JGT1 } & UDP-glucose:jasmonic acid & $\mathrm{R}-\mathrm{OH}$ & Hydroxy-substrate \\
\hline & glucosyltransferase 1 & $\mathrm{rpm}$ & Rotations per minute \\
\hline JOX & Jasmonate-induced oxygenase & RT & Retention time \\
\hline $\mathrm{k}_{\mathrm{cat}}$ & Maximal turnover number & $\mathrm{SA}$ & Salicylic acid \\
\hline $\mathrm{K}_{\mathrm{M}}$ & Michaelis-Menten constant & SA-GE & Salicylic acid-glucosyl ester \\
\hline LB & Luria-Bertani & SCF & Skp1/Cullin/F-box \\
\hline $\mathrm{LC}$ & Liquid chromatography & SDS & Sodium dodecyl sulfate \\
\hline \multirow[t]{2}{*}{ LC-HR-MS } & liquid chromatography coupled to & SEC & Size exclusion chromatography \\
\hline & high resolution mass spectrometry & $\mathrm{SP}$ & Straight phase \\
\hline \multirow[t]{2}{*}{ LC-MS } & Liquid chromatography coupled to & SRM & Single reaction monitoring \\
\hline & mass spectrometry & ST2a & Sulfotransferase $2 \mathrm{a}$ \\
\hline $\mathrm{LDH}$ & Lactate dehydrogenase & St-RA & 2-Oxothiazolidine-4-carboxylic \\
\hline LOX & Lipoxygenase & & acid \\
\hline M & Mol per liter & $\mathrm{t}$ & Reaction time \\
\hline$m / z$ & Mass-to-charge ratio & $\mathrm{T} 1$ & Transformants of the generation 1 \\
\hline mAU & Milli Absorption units & $\mathrm{T} 2$ & Transformants of the generation 2 \\
\hline \multirow[t]{2}{*}{$\mathrm{MeOH}$} & $\begin{array}{l}\text { Methanol of liquid } \\
\text { chromatography coupled to mass }\end{array}$ & $\mathrm{TAE}$ & $\begin{array}{l}\text { Tris/acetic acid/ } \\
\text { ethylenediaminetetraacetic acid }\end{array}$ \\
\hline & spectrometry grade & TBS & Tris buffered saline \\
\hline $\min$ & Minutes & T-DNA & Transfer DNA \\
\hline MR & Mutual rank & TEMED & Tetramethylethylenediamine \\
\hline MRM & Multiple reaction monitoring & TIC & Total ion chromatogram \\
\hline MS & Mass spectrometry & TLC & Thin-layer chromatography \\
\hline MS/MS & Mass spectrometry fragmentation & tracrRNA & Trans-activating crRNA \\
\hline MTBE & Methyl tert-buthylether & tri-OH-18:1 & $(10 E)-9,12,13-$ Trihydroxy-10- \\
\hline MW & Molecular weight & & octadecadienoic acid \\
\hline $\mathrm{NADH} / \mathrm{NAD}^{+}$ & Nicotinamide adenine dinucleotide & tri-OH-18:2 & (10E,15Z)-9,12,13-Trihydroxy- \\
\hline \multirow[t]{3}{*}{ NtSA-UGT } & Salicylic acid Uridine diphosphate & & 10,15-octadecadienoic acid \\
\hline & -dependent glycosyltransferase & $\mathrm{T}-\mathrm{TBS}$ & Tween-Tris buffered saline \\
\hline & from Nicotina tabacum & UDP & Uridine diphosphate \\
\hline $\mathrm{OD}_{600}$ & Optical density at $600 \mathrm{~nm}$ & UDP-Glc & Uridine diphosphate glucose \\
\hline $\mathrm{OH}-16: 0$ & Hexadecanol & UGT & Uridine diphosphate -dependent \\
\hline \multirow[t]{2}{*}{ OPC-6 } & 3-Oxo-2-pentenylcyclopentane-1- & & glycosyltransferase \\
\hline & hexanoic acid & UPLC & Ultra performance liquid \\
\hline \multirow[t]{2}{*}{ OPC-8 } & 3-Oxo-2-pentenylcyclopentane-1- & & chromatography \\
\hline & octanoic acid & UTP & Uridine triphosphate \\
\hline OPR2 & 12-Oxo-phytodienoate reductase 2 & $\mathrm{~V}$ & Reaction rate \\
\hline OPR3 & 12-Oxo-phytodienoate reductase 3 & $\mathrm{v} / \mathrm{v}$ & Volume per volume \\
\hline \multirow[t]{2}{*}{ OsSA-UGT } & $\begin{array}{l}\text { Salicylic acid Uridine diphosphate } \\
\text {-dependent glycosyltransferase }\end{array}$ & VvGT1 & $\begin{array}{l}\text { Glycosyltransferase UGT78A5 } \\
\text { from Vitis vinifera }\end{array}$ \\
\hline & from Oryza sativa & $w / v$ & Weight per volume \\
\hline p.d.u. & Procedure defined unit & ZYP-5052 & Auto-induction medium for protein \\
\hline $\mathrm{P} 450$ & Cytochrome P450 & & expression \\
\hline PAGE & Polyacrylamide gel electrophoresis & $\varepsilon$ & Extinction coefficient \\
\hline PAM & Protospacer adjacent motif & $\omega-O-G l c-16: 0$ & $\omega$-Glucosyl-hexadecanoic acid \\
\hline PAX1 & $\begin{array}{l}\text { Peroxisomal ATP-binding cassette } \\
\text { transporter } 1\end{array}$ & $\omega-\mathrm{OH}-16: 0$ & $\omega$-Hydroxy-hexadecanoic acid \\
\hline PCR & Polymerase chain reaction & & \\
\hline PDA & Photo diode array detector & & \\
\hline PEP & Phosphoenolpyruvate & & \\
\hline $\mathrm{pI}$ & Isoelectrical point & & \\
\hline Pip & Pipecolic acid & & \\
\hline PK & Pyruvate kinase & & \\
\hline POX & Peroxygenase & & \\
\hline PUFA & Polyunsaturated fatty acid & & \\
\hline qPCR & $\begin{array}{l}\text { Quantitative real-time polymerase } \\
\text { chain reaction }\end{array}$ & & \\
\hline
\end{tabular}




\section{LIST OF FIGURES}

Figure 1-1: Biosynthesis of jasmonic acid and jasmonoyl-isoleucine in plants.......................................

Figure 1-2: The LOX pathway is the major pathway of oxylipin formation .......................................

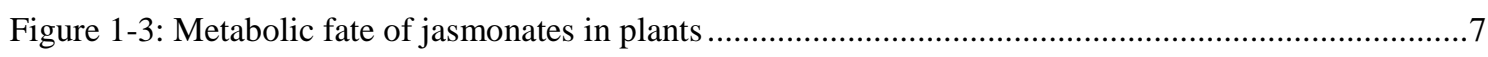

Figure 1-4: Nomenclature of UDP-dependent glycosyltransferases of family 1 ...................................11

Figure 1-5: Catalytic motifs of UDP-dependent glycosyltransferases ................................................ 13

Figure 1-6: Phylogenetic tree of UGTs of A. thaliana related in hormone homeostasis ...........................14

Figure 4-1: Expression of UGT76E1, UGT76E2, UGT76E11, UGT76E12, and UGT74F1 in A. thaliana

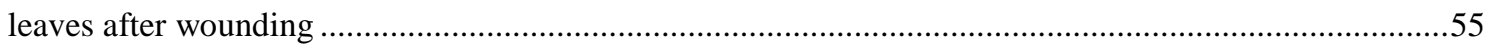

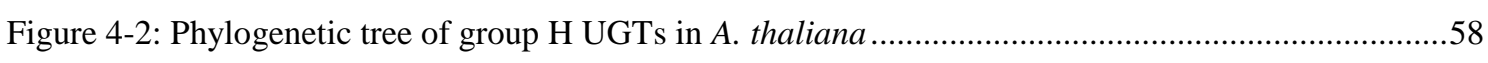

Figure 4-3: UGT76E1, UGT76E2, UGT76E11, and UGT76E12 show the catalytic motifs ....................58

Figure 4-4: UGT76E1, UGT76E2, UGT76E11, UGT7 E12, and UGT74F1 were expressed in E. coli....61

Figure 4-5: Optimization of the protein purification of UGT76E1 by affinity chromatography ..............63

Figure 4-6: Protein purification for UGT76E1, UGT76E2, UGT7E11, UGT76E12, and UGT74F1 ........64

Figure 4-7: LC-MS based activity assays of UGT76E1, UGT76E2, UGT7E11, UGT76E12, and

UGT74F1 with $\omega$-OH-hexadecanoic acid and 12-hydroxy-JA as substrates.........................................66

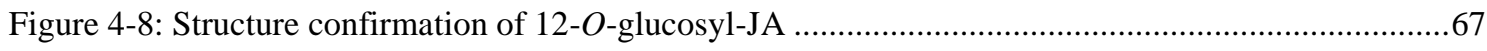

Figure 4-9: Optimum pH of UGT7E1, UGT76E2, UGT76E11, and UGT7E12 ….............................68

Figure 4-10: Substrate specificity of UGT7E1, UGT7E2, UGT76E11, UGT76E12, and UGT74F1 ........71

Figure 4-11: Search for native substrates of UGT76E1, UGT7E2, UGT76E11, UGT76E12, UGT74F1 by non-targeted ex vivo analysis

Figure 4-12: Activity of UGT76E1, UGT7E2, UGT76E11, UGT76E12, and UGT74F1 for 12-hydroxy-

JA, salicylic acid, and $\omega$-hydroxy-hexadecanoic acid in total metabolite extracts

Figure 4-13: Native substrates of UGT76E1, UGT7E2, UGT76E11, UGT76E12, UGT74F1 identified by

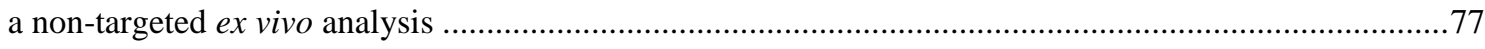

Figure 4-14: MS/MS analysis of the unknown substrate $\mathrm{C} 11 \mathrm{H} 18 \mathrm{O} 3$....................................................78

Figure 4-15: Scheme of a coupled spectrophotometric assay to record reactions of uridine diphosphate-

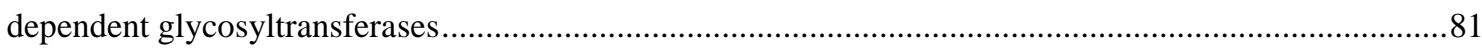

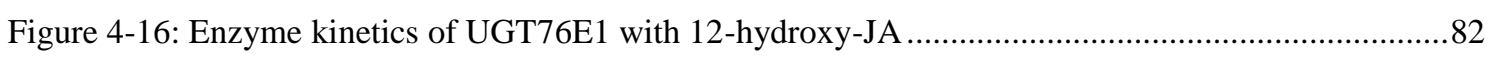

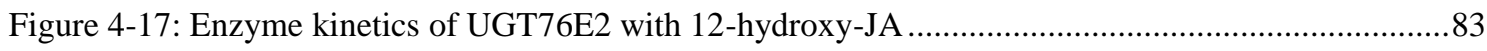

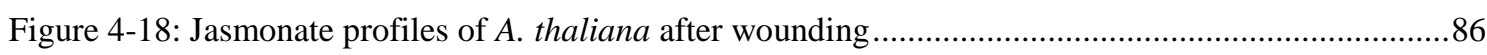

Figure 4-19: Vector maps of the CRISPR-Cas9 constructs for four UGT-target genes .........................87

Figure 4-20: Identification of a mutation in the ORF of $U G T 76 E 1$ by CRISPR-Cas9 activity ................89

Figure 5-1: UGT76E1, UGT76E2 complete the set of jasmonate-enzymes after wounding .....................99

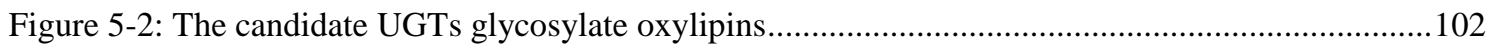

Supplemental figure 1: Phylogenetic tree of glycosyltransferases of A. thaliana ...................................117

Supplemental figure 2: Search for glucosyltransferase genes products by co-expression analysis ..........118 
Supplemental figure 3: Expression of UGT76E1, UGT76E2, UGT76E11, and UGT76E12 in A. thaliana leaves after wounding

Supplemental figure 4: Expression of UGT76E1 and UGT7E2 after abiotic stresses 120

Supplemental figure 5: Expression of UGT76E11 and UGT7E12 after abiotic stresses .121

Supplemental figure 6: Expression of UGT76E1, UGT76E2, UGT76E11, UGT76E12, and UGT74F1 in

Col-0 and JA-deficient mutant after wounding .122

Supplemental figure 7: Co-expression analysis of UGT76E1 and UGT76E2

Supplemental figure 8: Co-expression analysis of UGT76E11 and UGT76E12 124

Supplemental figure 9: Gene structures of UGT76E1, UGT76E2, UGT76E11, and UGT76E12 in

A. thaliana genome

Supplemental figure 10: Sequencing results of UGT76E1, UGT76E2, UGT76E11, UGT76E12, and $U G T 74 F 1$ integrated into the expression vector

Supplemental figure 11: Optimum temperature of UGT7E1, UGT76E2, UGT76E11, and UGT7E12 ....127

Supplemental figure 12: UGT76E12 shows side activity towards ethanol 128

Supplemental figure 13: 12-carboxy-JA was identified in leaves after wounding................................130

Supplemental figure 14: 12-O-glucosyl-jasmonoyl-isoleucine is not detected in plants ........................131

Supplemental figure 15: Enzyme kinetics with 9- and 13-hydroxy-octadecatrienoic acid .....................133

Supplemental figure 16: Phytohormone profiles of A. thaliana after wounding ....................................134 


\section{LIST OF TABLES}

Table 3-1: Equipment 18

Table 3-2: Software 19

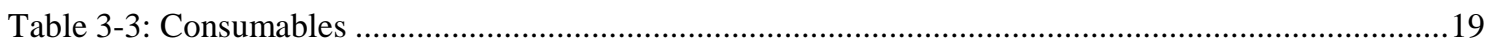

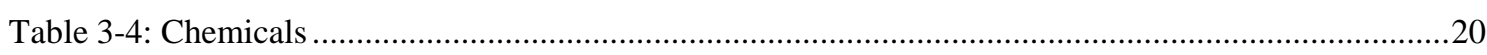

Table 3-5: Enzymes, size markers, antibodies, and molecular biological kits .......................................21

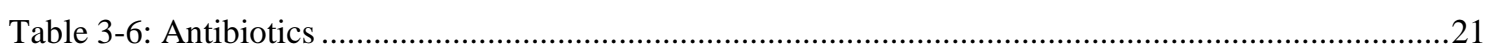

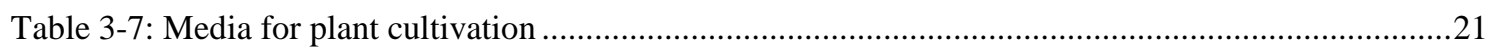

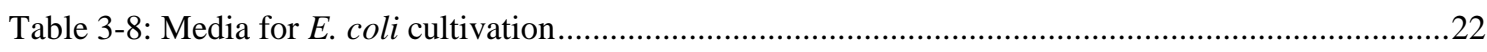

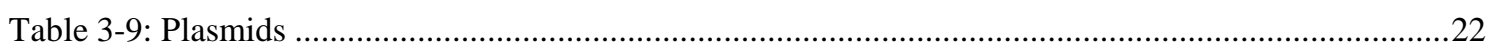

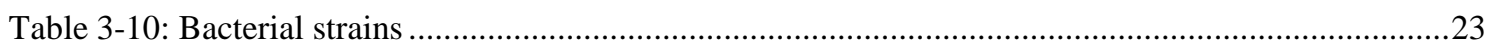

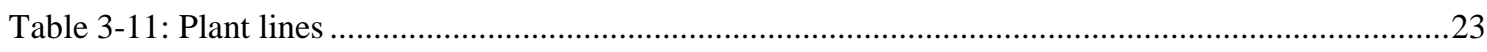

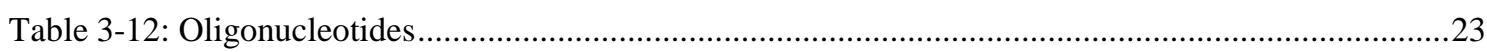

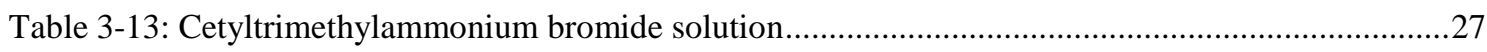

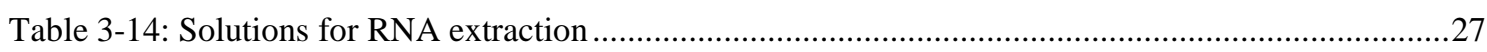

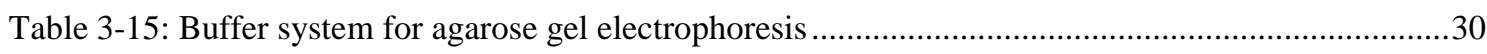

Table 3-16: Buffer systems for protein purification of UGT76E1 ....................................................37

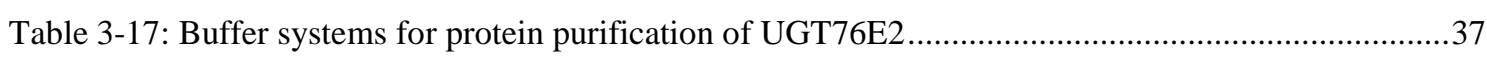

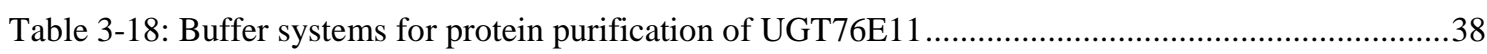

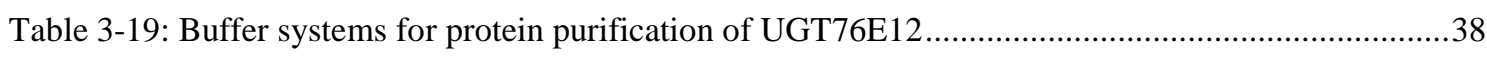

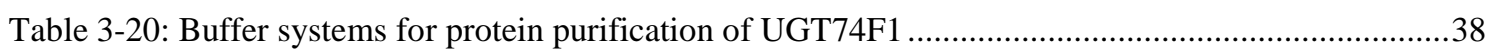

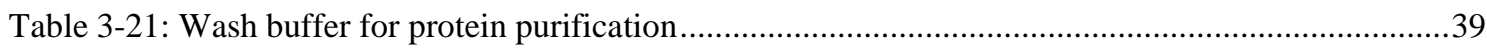

Table 3-22: Composition of 2x Laemmli sample buffer..........................................................................40

Table 3-23: Composition of $10 \%$ SDS-PAGE gel ..............................................................................40

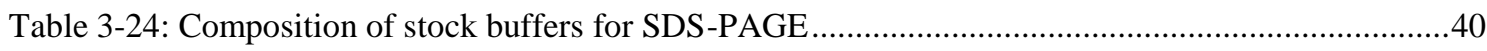

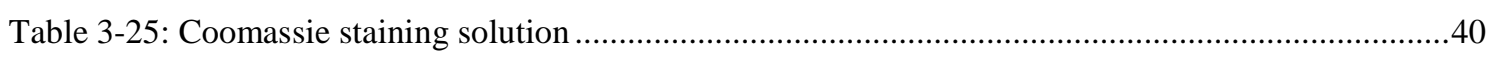

Table 3-26: Substances, which were tested as substrates for the UGTs. .............................................43

Table 3-27: Reaction mixture of the coupled spectrophotometric assay. .............................................44

Table 3-28: Internal standards for quantitative LC-MS/MS analysis ...................................................46

Table 3-29: Transitions of the multiple reaction monitoring mode of the LC-MS/MS analysis ...............48

Table 4-1: Search for glycosyltransferase genes as candidates for JA-glycosylation ...............................52

Table 4-2: Co-expression analysis of UGT76E1, UGT76E2, UGT76E11, and UGT76E12 _...................56

Table 4-3: Amino acid sequence comparison for UGT76E1, UGT76E2, UGT76E11, and UGT76E12 ....59

Table 4-4: Relative substrate preference of UGT76E1, UGT76E2, UGT76E11, UGT76E12, and UGT74F1

Table 4-5: Kinetic parameters of the UGT76E1, UGT76E2, UGT76E11, UGT76E12, and UGT74F1 towards their best substrates.....

Table 4-6: CRISPR target sequences for UGT76E1, UGT76E2, UGT76E11, and UGT76E12 ...............88 
Table 5-1: Comparison of kinetic parameters of UDP-dependent glycosyltransferases .

Supplemental table 1: Calculated protein parameters and predicted localizations for UGT76E1,

UGT76E2, UGT76E11, UGT76E12 and UGT74F1 125

Supplemental table 2: Chemical structures of the UGT-substances ...... 128

Supplemental table 3: Identification of specific metabolite markers by fragmentation .132 


\section{INTRODUCTION}

Providing food for the increasing human population is a critical issue nowadays. Increasing the yield is thus a common way to optimize the existing agricultural resources. However, humans have to share their revenues. Especially, feeding insects and pathogen infections cause losses in agriculture. Within optimizing agricultural processes, genetic and biochemical approaches help to understand plant defense as well as pathogen invasion and have already pushed the limits (Guo et alia (et al.), 2018). "A detailed understanding of plant immunity to arthropod herbivores will provide new insights into basic mechanisms of chemical communication and plant-animal coevolution and may also facilitate new approaches to crop protection and improvement" (Howe \& Jander, 2008). Thereby, plants actively respond to threats by producing toxins, defensive proteins and emitting volatiles that recruit insect predators. Interestingly, insects and injured plant cells initiate the defense responses themselves. These initial stimuli are processed by conserved pathways of plant hormones and the group of jasmonates promote many defense responses to a broad spectrum of insects and pathogens (Koo, 2018).

\subsection{JASMONATES AS PLANT HORMONES}

In addition to defense against biotic attackers, jasmonates are involved in abiotic stress responses (Farmer, 2007; Wasternack et al., 1998) and influence developmental processes like flower development and root growth (Ueda \& Kato, 1980; Yoshihara et al., 2014). Jasmonates are molecules that derive from the core molecule jasmonic acid (JA) (Wasternack et al., 1998). They may have similar evolutionary origin and biosynthetic similarities to eicosanoids, which act in mammalian wound and inflammatory responses (Koo, 2018). Eicosanoids like leukotrienes and prostaglandins have been studied extensively due to their clinical importance (Bensinger \& Tontonoz, 2008).

To study the formation of jasmonates and processes regulated by them in plants, mechanical tissue damage is often used to elicit the core of inducible defense responses (Koo \& Howe, 2009). Thereby, the jasmonate pathway can be stimulated in a controlled and reproducible way. On top of this, jasmonates induce systemic responses by either acting as a mobile signal or being synthesized de novo at the distal site (Gasperini et al., 2015; Glauser et al., 2009; Koo \& Howe, 2009).

Initially, the odorant molecule JA-methyl ester (JA-ME) was isolated from Jasminium grandiflorum flowers (Demole et al., 1962), which gave the name to the whole class of jasmonates. Since then, many aspects of the biosynthesis, signal perception, hormonal activity and different physiological effects have been elucidated (Wasternack, 2015). 


\subsubsection{JA-BIOSYNTHESIS}

A variety of oxidized fatty acids (FAs) - so-called oxylipins - have functions in intra- and intermolecular signaling in animals, plants and fungi (Andreou et al., 2009; Wasternack \& Feussner, 2018). In plants, jasmonoyl-isoleucine (JA-Ile) - the active form of JA - is the major oxylipin acting as phytohormone.

The synthesis of oxylipins may start with the peroxidation of polyunsaturated fatty acids (PUFAs). In case of JA, an unspecific glycerolipase may release $\alpha$-linolenic acid ((9Z,12Z,15Z)9,12,15-octadecatrienoic acid) from one of the two positions of a galactolipid of the plastidial membrane (Figure 1-1) (Koo, 2018). This molecule is oxygenated at the C13 to 13-hydroperoxyoctadecatrienoic acid (13-HPOT) by specific 13-linoleate lipoxygenase (LOX) enzymes (13LOX). In A. thaliana leaves, LOX2 is responsible for the bulk of JA synthesis in the first hour post wounding (hpw) (Glauser et al., 2009). However, in this plant up to four isoenzymes can contribute to JA formation (Caldelari et al., 2011; Chauvin et al., 2012). In addition, two other LOXs (9-LOX) specifically catalyze 9-hydroperoxy-octadecatrienoic acid (9-HPOT) from $\alpha$ linolenic acid. Altogether, this LOX-pathway feeds the substrate pool of this branch with 9- and 13-HPOT (Blée, 2002). Several enzymes may compete for this substrate pool (Figure 1-2) (Feussner \& Wasternack, 2002). In plants, a specific group of cytochrome P450 (P450) enzymes of the CYP74 family primarily metabolizes the LOX-products. As a member of this family, a hydroperoxide lyase (HPL) cleaves the hydroperoxides and produces green-leaf volatiles, which may attract beneficial predators to fight against herbivores (Chehab et al., 2008). Noticeable, the A. thaliana accession Columbia 0 (Col-0) is natural HPL-mutant (Duan et al., 2005), which promotes JA-production by elevated substrate availability (Chehab et al., 2008). Furthermore, glycosylated oxylipins were identified (Kai et al., 2010). Such molecules may be formed from hydroxy or carboxy derivatives such as $(10 E, 12 Z, 15 Z)-9$-hydroxy-10,12,15-octadecatrienoic acid (9-HOT) and (9Z,11E,15Z)-13-hydroxy-9,11,15-octadecatrienoic acid (13-HOT), which are synthetized from $\alpha$-linolenic acid by LOX and reductase activity (Figure 1-2).

The second member of the CYP74 family is allene oxide synthase (AOS). In A. thaliana, a single copy gene is detectable in its genome. This enzyme reaction may be the first step with 13-HPOT as substrate of JA-biosynthesis (Figure 1-2) by producing an unstable allene oxide 12,13-epoxyoctadecatrienoic acid (12,13-EOT, Figure 1-1). The allene oxide cyclase (AOC) subsequently converts 12,13-EOT to the stable cis-(+)-12-oxo-phytodienoic acid (12-OPDA). Those three reactions take place in the plastid, from where 12-OPDA gets exported by a yet unknown protein and imported into the peroxisome by the peroxisomal ATP-binding cassette transporter 1 (PAX1) (Theodoulou et al., 2005). There, the double bond of 12-OPDA within the cyclopentene ring is reduced to yield 3-oxo-2-pentenylcyclopentane-1-octanoic acid (OPC-8) by the 


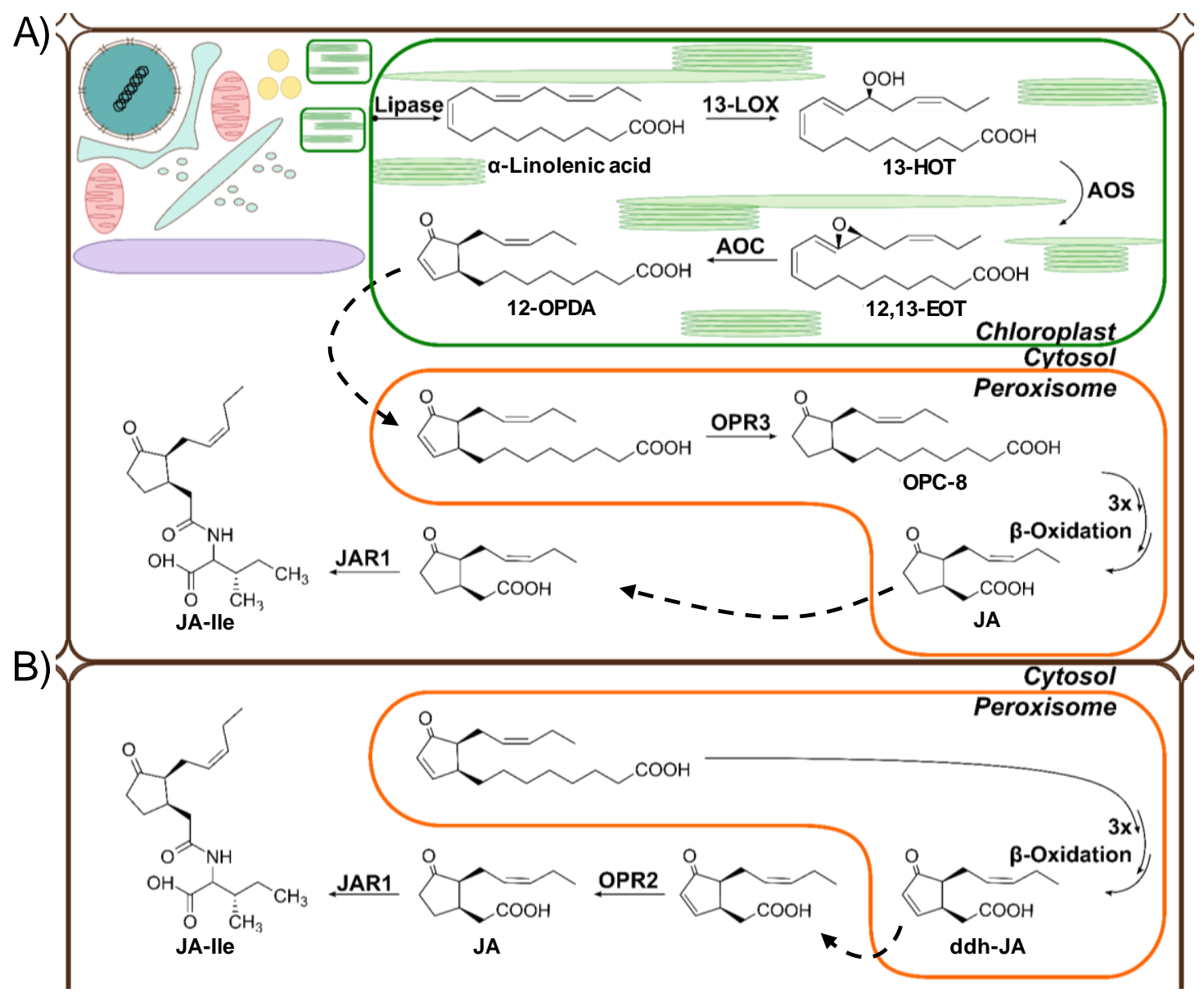

Figure 1-1: Biosynthesis of jasmonic acid and jasmonoyl-isoleucine in plants

The biosynthesis of jasmonic acid (JA) is spread over three cell compartments. A) Linolenic acid, released from the inner plastidial membrane by a lipase, is oxidized to 13-hydroperoxy-octadecatrienoic acid (13-HPOT) by a 13-lipoxygenase (13-LOX). 13-HPOT is dehydrated to 12,13-epoxyoctadecatrienoic acid (12,13-EOT) by the allene-oxide synthase (AOS) and cyclized to the 12-oxophytodienoic acid (12-OPDA) by the allene-oxide cyclase (AOC). 12-OPDA is transported from the chloroplast into the peroxisome where it is reduced to 3-oxo-2-pentenyl-cyclopentane-1-octanoic acid (OPC-8) by the oxo-phytodienoate reductase isoform 3 (OPR3). Shorted in three rounds of $\beta$-oxidation, JA is formed and exported into the cytoplasm. There, it gets hormonal activity by the conjugation to isoleucine by jasmonic acid resistant 1 (JAR1) forming jasmonoyl-isoleucine (JA-Ile). B) An alternative JA pathway lacks the reduction in the peroxisome yielding didehydro-JA (ddh-JA) after the $\beta$-oxidations. Final reduction of cytosolic ddh-JA to JA is performed by OPR isoform 2 (OPR2). Transports over biological membranes are indicated by dashed arrows. Figures modified from Wasternack \& Hause, 2013 and Chini et al., 2018.

12-oxo-phytodienoate reductase isoform 3 (OPR3). Finally, three rounds of $\beta$-oxidation shorten OPC-8 to yield JA. Each $\beta$-oxidation cycle includes activation by the OPC- 8 Coenzyme A ligase 1 (Koo et al., 2006), dehydrogenation, hydration, and oxidation by the three enzymes of the FA$\beta$-oxidation (Figure 1-1A).

Another route in JA synthesis is the initial release and peroxidation of roughanic acid (16:3 [n3]) leading to dinor-12-OPDA (dnOPDA) (Weber et al., 1997). Parallel to 12-OPDA, this molecule is thought to be translocated and reduced by OPR3 (Chini et al., 2018) yielding JA via 3-oxo-2-pentenylcyclopentane-1-hexanoic acid (OPC-6). Recently, another alternative path was 
identified, which lacks the initial reduction of 12-OPDA to OPC-8 in the peroxisome. Here, the 12-OPDA undergoes the three cycles of $\beta$-oxidation yielding didehydro-JA (ddh-JA), which, in turn, gets exported to the cytoplasm and is reduced to JA by OPR isoform 2 (OPR2, Figure 1-1B) (Chini et al., 2018).

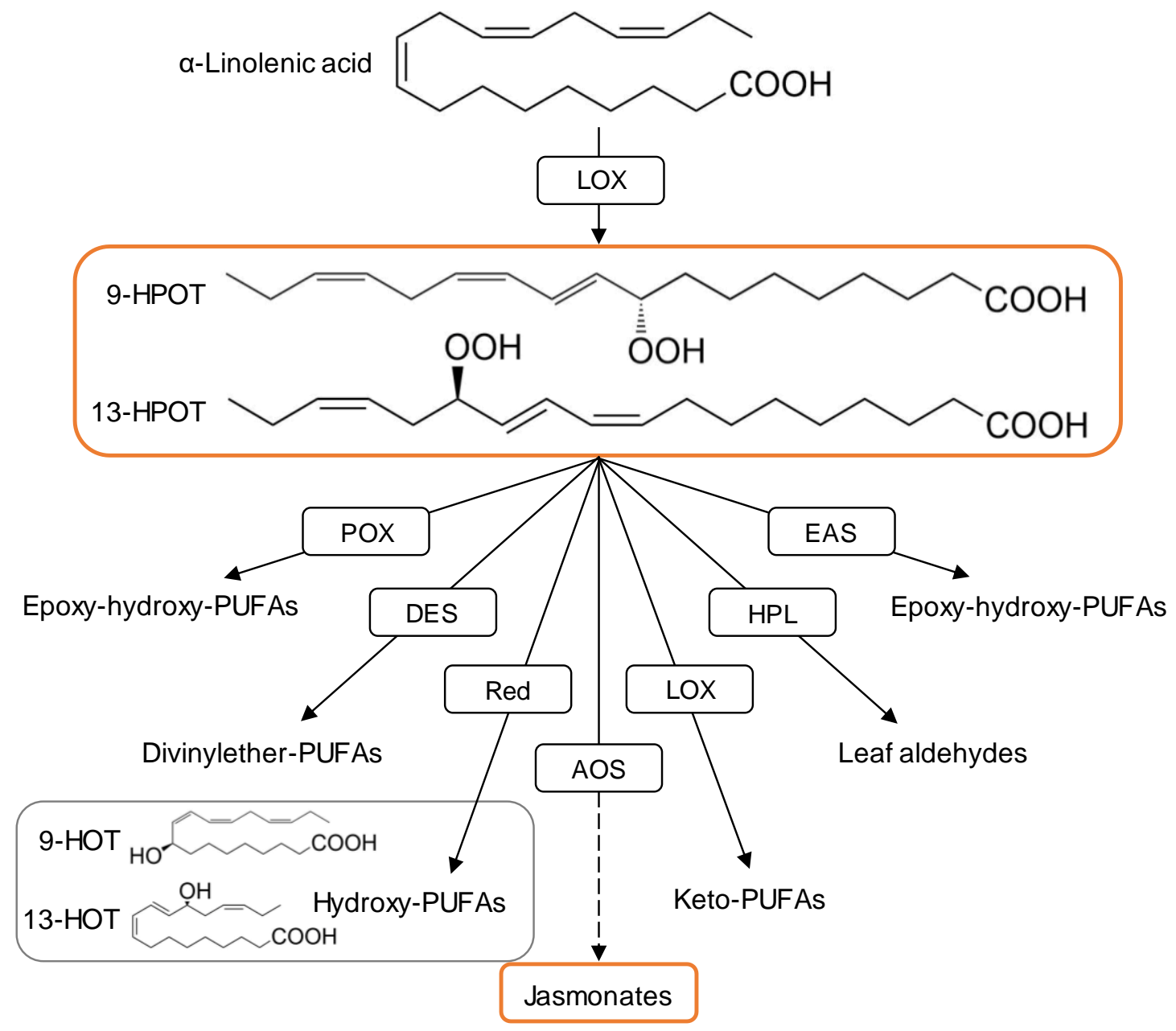

Figure 1-2: The LOX pathway is the major pathway of oxylipin formation

Lipoxygenases (LOX) oxygenate polyunsaturated fatty acids (PUFAs) such as $\alpha$-linolenic acid to the 9LOX- and 13-LOX-derived hydroperoxides, 9-hydroperoxy-octadecatrienoic acid (9-HPOT) or 13hydroperoxy-octadecatrienoic acid, (13-HPOT). Those molecules can be metabolized to diverse oxylipins: epoxy-hydroxy PUFA by a peroxygenase (POX), divinyl ether PUFAs by a divinyl ether synthase (DES), hydroxy PUFAs by a reductase (Red), jasmonates by an allene oxide synthase (AOS), keto PUFAs by a LOX, leaf aldehydes by a hydroperoxide lyase (HPL), and to epoxy-hydroxy PUFAs formed by an epoxy alcohol synthase (EAS). Figure is modified from Feussner \& Wasternack, 2002.

\subsubsection{JA-ILE SIGNALING}

In plant defense, JA-Ile orchestrates a rapid defense towards herbivores, wounding or necrotrophic pathogen attacks. Signal transduction pathways like calcium ion fluxes and phosphorylation cascades assist jasmonate signaling (Howe \& Jander, 2008). In A. thaliana, JA 
levels rise within $30 \mathrm{~s}$ in the local leaf and in $120 \mathrm{~s}$ in the systemic leaves (Glauser et al., 2009). For its activation, JA is conjugated with the amino acid isoleucine to form JA-Ile by the jasmonic acid-amido synthetase, named JA resistance 1 (JAR1), a member of the acyl acid-amido synthetase family Gretchen Hagen 3 (GH3, GH3.11, Figure 1-3) (Westfall et al., 2012). It derepresses transcription of the JA-responsive genes (Wasternack \& Hause, 2013). Transcription activators, exempli gratia (e. g.) the leucine-zipper MYC2, are constitutively bound to promoter regions of these genes which are in turn repressed by jasmonate zinc-finger inflorescence meristem domain (JAZ) proteins. Additionally, the gene transcription is successfully down regulated by the interaction of the co-repressors novel interactor of JAZ in complex with topless (Pauwels et al., 2010). Upon de-repression of JA-responsive genes, JA-Ile enables molecular binding of coronatine insensitive 1 (COI1) - as F-box ligase of the Skp1/Cullin/F-box(COI1) $\left(\mathrm{SCF}^{\mathrm{COI1}}\right)$ complex - to JAZ. Likewise, the JA-repressors are targeted for poly ubiquitination and protein degradation by the proteasome (Wasternack \& Hause, 2013). Subsequently, MYC2 and its bound co-activator mediator 25 can activate JA-responsive gene expression. Such genes include new JAZ and MYC2, which suppress further transcription in the absence of an additional JA-Ile signal as negative feedback regulation (Koo et al., 2014). The real situation in the nucleus involves more regulative proteins like histone acetyltransferases or additional repressors competitive for MYC2-binding called jasmonate associated MYC2-like proteins (Wasternack \& Song, 2017). This complex regulation directs the interaction with other phytohormones and balances growth and defense related pathways (see 1.1.4) (confer (cf.) Guo et al., 2018; Wasternack \& Song, 2017),

So far, two transporters have been described that are involved in this pathway: PXA1 and the jasmonic acid transporter 1 (JAT1). JAT1 has dual function. It imports JA-Ile into the nucleus and transports JA or JA-Ile through the plasma membrane (Li et al., 2017; Nguyen et al., 2017). Further, intercellular transport of both maybe mediated by the multifunctional glucosinolate transporter 1 (GTR1) transporting gibberellins and jasmonates (Ishimaru et al., 2018; Saito et al., 2015).

Concerning the JA-precursor 12-OPDA and its amino acid conjugate 12-OPDA-Ile, it has been discussed that they may have their individual set of responsive genes (Arnold et al., 2016). The structural and metabolic relation of 12-OPDA-Ile to JA-Ile suggests similar behavior but, for 12 OPDA-Ile, neither the enzymatic conjugation, the transport mechanism, nor the perception have been studied in A. thaliana yet. However, in early land plants like bryophytes, the active compound JA-Ile is not present. In liverworts for example, the alternative precursor dnOPDA stimulates COI1-JAZ interaction within the molecular machinery (Monte et al., 2018). Furthermore, several jasmonate derivatives were tested to have activating or inactivating functions (see Figure 1-3) (Miersch et al., 2008; Wasternack \& Feussner, 2018). 
As a result, JA-Ile signaling may stimulate the synthesis of chemicals that act as insect deterrents, toxins, or anti-nutritive substances (Wu \& Baldwin, 2010). Such compounds are often stored in specialized defensive organs like trichomes (Yoshida et al., 2009). It also promotes defense by deposition of callose, surface lipids, and phenolics (Koo, 2018). In addition, jasmonates promote the emission of volatiles that may alert neighboring plants or attract insect predators (Kessler \& Baldwin, 2001). Physiologically, the JA-Ile pathway readjusts growth and development for defense (Guo et al., 2018).

\subsubsection{JA-ILE INACTIVATION}

As a phytohormone, JA-Ile reprograms plants to defense meaning signal-termination is important to restore growing conditions (Guo et al., 2018; Koo, 2018). Other phytohormones are also conjugated to amino acids. In contrast to JA-Ile, auxin is inactivated by conjugation whereas the free molecule is active. However, GH3 enzymes catalyze the reaction in analogy to JAR1 (Staswick \& Tiryaki, 2004). For JA-Ile, there are at least two ways to terminate the signal: deconjugation of the active conjugate and oxidation of the molecule at the $\omega$-terminus of the pentenyl side chain. However, both pathways may overlap and co-operate in inactivating the JAIle signal (Figure 1-3).

So far, the oxidation of the active JA-Ile to the 12-hydroxy-JA-Ile (12-OH-JA-Ile) may be the initial and direct modification for the inactivation. Further oxidation yields 12-carboxy-JA-Ile (12-COOH-JA-Ile) (Heitz et al., 2012). All three oxidation states - JA-Ile, 12-OH-JA-Ile, and 12COOH-JA-Ile - can be de-conjugated by the amidohydrolase indole-3-acetic acid (IAA)-alanine resistant 3 (IAR3, Figure 1-3) (Zhang et al., 2016). In addition, IAA-leucine resistant-like 6 (ILL6) is capable to cleave off the amino acid from JA-Ile and 12-OH-JA-Ile (Figure 1-3) (Widemann et al., 2013; Zhang et al., 2016). An ill6iar3 double mutant with increased JA-Ile/12$\mathrm{OH}-\mathrm{JA}-\mathrm{Il}$ levels and overexpressing lines with reduced levels confirm the redundant action of ILL6 or IAR3 in planta (Zhang et al., 2016).

However, cleavage of JA-Ile to JA alone retains the potential of a re-activation whereas oxidation to 12-OH-JA-Ile indeed reduces activity towards transcriptional activation (Koo et al., 2014; Koo et al., 2011). Generally, oxidation is a common step of inactivating phytohormones and is often catalyzed by $\mathrm{P} 450$ enzymes. For instance, hydroxylation of abscisic acid (ABA), gibberellic acid (GA) and brassinosteroids are essential steps in signal termination (Mizutani \& Sato, 2010). Metabolomic data have shown that oxidized jasmonates like 12-OH-JA-Ile and 12-COOH-JA-Ile accumulate when JA-Ile levels decrease (Bruckhoff et al., 2016). Three P450s - CYP94B1, CYP94B3, and CYP94C1 - have been identified to oxidize JA-Ile with different specificities (Heitz et al., 2012; Kitaoka et al., 2011; Koo et al., 2014; Koo et al., 2011; Widemann et al., 2016). CYP94B1 and CYP94B3 preferentially form 12-OH-JA-Ile during plant defense and 
CYP94C1 catalyzes the reaction in flower maturation (Widemann et al., 2016). Here again, the three P450s act redundantly (Bruckhoff et al., 2016; Koo et al., 2014). However, CYP94C1 exclusively forms 12-COOH-JA-Ile (Figure 1-3) (Bruckhoff et al., 2016; Widemann et al., 2016). Besides its accumulation after wounding, 12-COOH-JA-Ile is formed during flower opening (Widemann et al., 2016). However, possible functions of the different oxidized JA-Ile derivatives have to be investigated.

Subsequent de-conjugation of 12-OH-JA-Ile may yield 12-hydroxy-JA (12-OH-JA), which did not exhibit JA-typical responses like inhibition of root growth, germination or expression of JA responsive genes (Gidda et al., 2003; Miersch et al., 2008). Although residual amounts of 12 $\mathrm{OH}-\mathrm{JA}$ were present in different mutant lines theoretically impaired in 12-OH-JA formation by

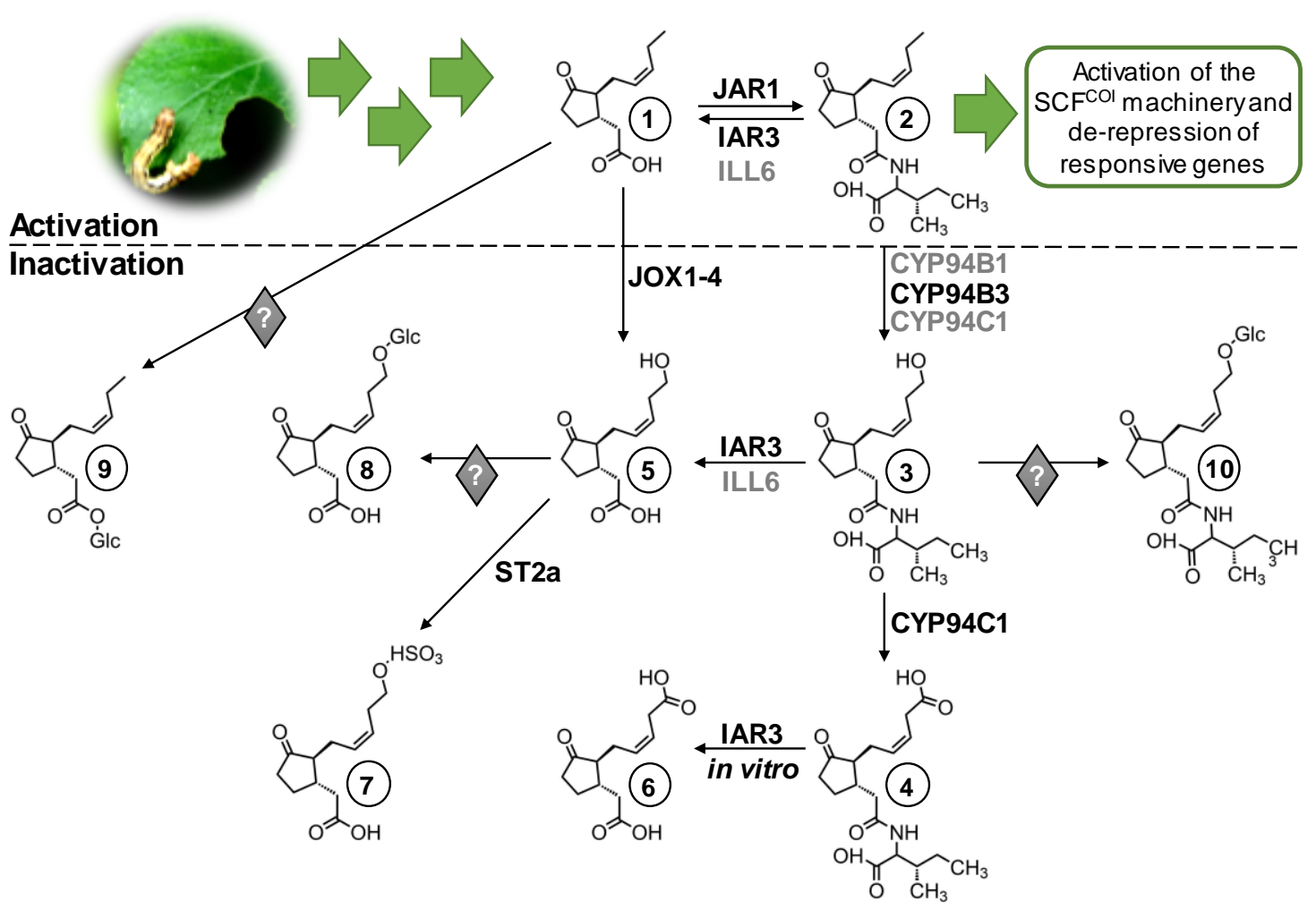

Figure 1-3: Metabolic fate of jasmonates in plants

Wound stimuli like feeding insects lead to the activation of jasmonic acid (JA, 1) biosynthesis. JA gets active as hormone by the conjugation to isoleucine. Jasmonoyl-isoleucine (JA-Ile, 2) stimulates derepression of JA-responsive genes via proteasomal degradation. The amounts of JA and JA-Ile in the cell are controlled by $\omega$-oxidations and/or de-conjugation of the isoleucine-moiety. CYP94B1, CYP94B3, and CYP94C1 oxidize JA-Ile to 12-hydroxy-JA-Ile (12-OH-JA-Ile, 3) and CYP94C1 to 12-carboxy-JA-Ile (12-COOH-JA-Ile, 4). JA-Ile, 12-OH-JA-Ile, and 12-COOH-JA-Ile get de-conjugated by IAA-alanineresistant 3 (IAR3) and IAA-leucine-resistant-like 6 (ILL6) forming JA, 12-hydroxy-JA (12-OH-JA, 5) and 12-carboxy-JA (12-COOH-JA, 6), respectively. JA is oxidized to 12-OH-JA by jasmonate-induced oxidases 1 to 4 (JOX1-4). 12-OH-JA gets modified to 12-OH-JA sulfate (12- $\left.\mathrm{HSO}_{4}-\mathrm{JA}, 7\right)$ by sulfotransferase 2a (ST2a) or glycosylated to 12-O-glucosyl-JA (12-O-Glc-JA, 8). Question marks indicate so far unknown enzymes for the reactions to 12 - $O$-Glc-JA, jasmonic acid-glucosyl ester (JA-GE, 9), and 12-O-glucosyl-JA-Ile (10). Scheme modified from Wasternack \& Hause, 2013, additions from Caarls et al., 2017; Kitaoka et al., 2014; Widemann et al., 2016; Zhang et al., 2016. Photo was taken from Iven et al., 2014. 
serial hydroxylation and de-conjugation of JA-Ile ( $c f$. Bruckhoff et al., 2016; Widemann et al., 2013; Zhang et al., 2016), this path was the only route to form 12-OH-JA known until recently. After the direct oxidation of JA had been suggested (Bruckhoff et al., 2016), the responsible enzymes were independently identified twice (Caarls et al., 2017; Smirnova et al., 2017). These jasmonate-induced oxygenase 1 to 4 (JOX1-4) belong to the family of 2-oxoglutarate Fe(II)dependent oxygenases (Figure 1-3) (Caarls et al., 2017). The respective quadruple mutant showed elevated JA-levels and reduced levels of 12-OH-JA resulting in higher resistance to a necrotrophic fungus and feeding insects. Although induced by JA, JOX1-4 down-regulate the JA-Ile-dependent responses (Caarls et al., 2017) like it has been observed for their metabolic product 12-OH-JA before (Miersch et al., 2008). Trying to explain this dual synthesis routes of 12-OH-JA, Smirnova and co-workers suggested specificity of the de-conjugation path in response to wounding and the oxidative path for necrotrophic pathogen attack. Besides its dual synthesis paths, 12-OH-JA can be modified by two different ways (Figure 1-3). On the one side, the specific sulfotransferase 2a (ST2a) is known to form 12-hydroxy-JA sulfate (12-HSO 4 -JA) (Gidda et al., 2003). On the other side, the 12-hydroxy group can be glycosylated to form 12-O-glucosyl-JA (12-O-Glc-JA) (Wasternack \& Feussner, 2018). Here, the responsible enzymes are still elusive (Koo, 2018; Wasternack \& Feussner, 2018). Similar to $12-\mathrm{OH}-\mathrm{JA}, 12-\mathrm{HSO}_{4}-\mathrm{JA}$ and $12-\mathrm{O}$-Glc-JA may be part of the inactivation process of the JA-Ile defense (Gidda et al., 2003; Miersch et al., 2008). All three metabolites (12-OH-JA, 12- $\mathrm{HSO}_{4}-\mathrm{JA}$, and 12-O-Glc-JA) have been identified in many plant species in an organ-specific manner (Miersch et al., 2008; Seto et al., 2009). In addition, 12-OHJA has been identified as tuber-inducing compound in Solanum tuberosum, which is reflected in its trivial name tuberonic acid (Yoshihara et al., 2014).

In analogy to the oxidation products of conjugated jasmonates, also JA, 12-OH-JA, and 12$\mathrm{COOH}-\mathrm{JA}$ are present in A. thaliana. The metabolite 12-COOH-JA has been found in open flowers of A. thaliana (Bruckhoff et al., 2016). Although, the biosynthesis is unknown the abundance of 12-COOH-JA is connected to CYP94C1-activity (Bruckhoff et al., 2016). Furthermore, IAR3 is capable to de-conjugate 12-COOH-JA-Ile to 12-COOH-JA in vitro (Figure 1-3) (Zhang et al., 2016). On top of this, the abundance of 12-COOH-JA in open flowers might be connected to the accumulation of 12-COOH-JA-Ile upon flower opening (Widemann et al., 2016). Hence, 12-COOH-JA is likely to be another catabolic derivative by serial oxidation and de-conjugation of jasmonates.

In summary, six different inactivating metabolites are found around the two active molecule JAIle. Mainly, two catabolic routes terminate the defense signal by terminal oxidation and deconjugation. Three different oxidation states have been identified for the core molecule JA and the active form JA-Ile. Within the catabolic fate, 12-OH-JA has an emphasized position, since it is produced in two ways (oxidation of JA and de-conjugation of 12-OH-JA-Ile) and metabolized 
in two ways to $12-\mathrm{HSO}_{4}-\mathrm{JA}$ and $12-\mathrm{O}$-Glc-JA. Surprisingly, no individual metabolic functions of any of the catabolic derivatives has been identified yet.

\subsubsection{JASMONATE CROSSTALK}

Major plant processes like growth, development, and defense are orchestrated by different phytohormones. Therefore, a precise balance of these general processes and the actually needed pathways is essential for an effective plant growth and metabolism. A complex network of signal transduction pathways regulated by the different phytohormones regulates and balances the interaction of plants with their environment (Guo et al., 2018). In this respect, the enzymes of the different pathway are good examples how this crosstalk is achieved. As mentioned above, the amidohydrolases IAR3 and ILL6 were initially identified and named as auxin hydrolases (Zhang et al., 2016) however they use JA-Ile as substrate as well. Besides JAR1, another member of the GH3 family (AtGH3.5) conjugates auxin, benzoic acid (BA), and salicylic acid (SA) (Westfall et al., 2016). Such broad substrate tolerances for different plant hormones allow common modification patterns of different phytohormones leading to a complex and fine-tuned crosstalk between the different phytohormones that can influence each other. In the case of jasmonates, the crosstalk with other phytohormones or day and night signaling pathways regulates the balance between growth and defense (Guo et al., 2018; Pieterse et al., 2009).

JA-Ile inhibits root growth by crosstalk with auxin (Yoshihara et al., 2014) but also promotes the activation of auxin biosynthesis and vice versa (Koo, 2018). On top of this, both pathways share a similar genetic regulation via a SCF complex-mediated degradation of repressors (Koo, 2018). This might explain the overlapping activities of JA- or auxin-related enzymes. A crosstalk between JA-Ile and GA may be synergistic in stamen development but also antagonistic during plant growth and defense (Wasternack \& Hause, 2013). Here, the main regulators JAZ and DELLA repress each other (Koo, 2018). Next, the interaction of JA-Ile and ABA is mostly antagonistic due to their roles in different stress responses - water control after drought or heat is regulated by $\mathrm{ABA}$, while the wound response is controlled by JA-Ile. However, the crosstalk of JA-Ile and ABA can also have a synergistic character since wounding may cause water loss and ABA receptors are expressed via JA-Ile in Nicotina tabacum (N. tabacum) and A. thaliana (Lackman et al., 2011). In defense, JA-Ile and ethylene generally act synergistically in response to necrotrophic pathogens (Pieterse et al., 2013; Xu et al., 1994). Here, JAZ-degradation activates both, JA-Ile and ethylene controlled responses (Zhu et al., 2011). Anyhow, the best studied phytohormone crosstalk is between JA-Ile and SA. Both hormones act in plant defense but SA is involved in the response towards biotrophic pathogens, while JA-Ile reacts towards necrotrophic pathogens and tissue damage. In nature, plants often have to face both infections simultaneously 
and JA-Ile and SA levels are well balanced until one particular stimulus pushes the response towards one defense pathway (Wasternack \& Hause, 2013).

A complex network of interaction between JA-Ile and different other phytohormones may be assisted by chemical modifications, which potentially change the signaling character of JA-Ile. In case of jasmonates, different modifications are known to take place in plants, like conjugation, oxidation, de-conjugation, methylation, sulfation, and glycosylation (see Figure 1-3) (Iven et al., 2014) but most of the physiological roles of such modifications are elusive (Koo, 2018). In contrast to this, many specific enzymes catalyzing the different modifications of jasmonates are well studied. The only modification where the enzymes enzymatic are not known yet is the glycosylation of different jasmonates (JA, 12-OH-JA, 12-OH-JA-Ile, Figure 1-3).

\subsection{GLYCOSYLTRANSFERASES IN PHYTOHORMONE HOMEOSTASIS}

Glycosylated forms of all phytohormones except ethylene are well known in A. thaliana (Bowles et al., 2006). All individual steps in phytohormone-metabolism are regulated and balanced by specific modifications whereby glycosylation is often the final step (Vogt \& Jones, 2000). Glycosylation increases the solubility of lipophilic compounds like anthocyanin pigments (Vogt $\&$ Jones, 2000). Furthermore, it promotes metabolic transport and likewise changes the inter- or intracellular localization of the metabolites, $e . g$ the glycosylated anthocyanin pigments are stored in the vacuole. In combination with a specific hydrolase enzyme, glycosylated molecules can serve as reversible storage forms. Whereas, without a hydrolase the molecule can be trapped in the organelle to regulate the cellular pool of bioactive compounds. In both cases, the activity of glycosyltransferases regulate the bioactivity and the homeostasis of specialized metabolites and phytohormones. Hereby, the responsible UGT is often controlled on the transcriptional level to regulate its mode and time of action (Bowles et al., 2006; Ross et al., 2001; Vogt \& Jones, 2000). In analogy, glycosylation of specialized molecules is part of a three-step detoxification-strategy: Phase $\mathrm{I}$ is an activation of the molecule by oxidation or hydrolytic cleavage. Phase II is the glycosylation itself. It is a second layer of chemical modification, which prepares the compound for elimination from the cytosol. Phase III is the transport of the modified molecule into the vacuole or the apoplast (Coleman et al., 1997). Besides the plants vacuole, the apoplast is another storage site, which accumulates inert derivatives for further storage or degradation (Dietz et al., 2000).

Glycosyltransferase enzymes catalyze the transfer of a hexose-moiety to a chemical. There are different classifications of glycosyltransferases known. The database of carbohydrate-active enzyme (CAZy) ranks all glycosyltransferases known into 106 sequence-based families by their co-substrate usage $e . g$. nucleotide diphosphate-sugars, nucleotide monophosphate-sugars or 
sugar phosphates (http://www.cazy.org/, access 20.08.2018) (Campbell et al., 1997). Glycosylation of natural products and small lipophilic compounds is catalyzed by glycosyltransferases of the family 1 . This is the biggest family of all glycosyltransferases known and uses uridine diphosphate (UDP)-activated sugars for catalysis (Bowles et al., 2006). This subgroup was then used as basis for more detailed groupings (Campbell et al., 1997): All glycosyltransferases using UDP-activated sugars are called UDP-dependent-glycosyltransferases (UGTs) and are grouped into one superfamily. The UGT-superfamily is divided into families by at least $40 \%$ amino acid sequence homology. Here, the families 71 to 100 are specific for plants. These 30 UGT families are further separated into subfamilies by at least $60 \%$ homology and are classified by individual numbers (Figure 1-4) (Mackenzie et al., 1997). This nomenclature will be used throughout this work to describe the analyzed glycosyltransferases.

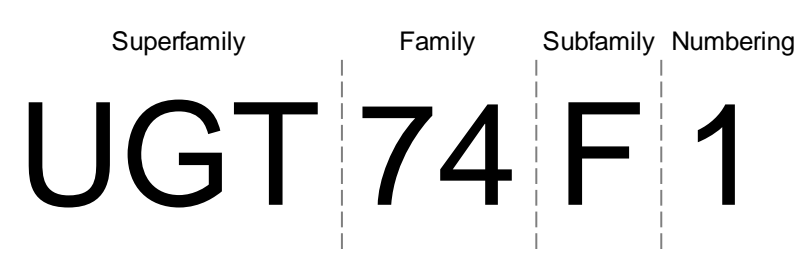

Figure 1-4: Nomenclature of UDP-dependent glycosyltransferases of family 1

The scheme illustrates the systematic nomenclature of the superfamily of UDP-dependent glycosyltransferases of the family 1 (UGTs). Further grouping is achieved in families with $\geq 40 \%$ and in subfamilies with $\geq 60 \%$ amino acid sequence homology. Lastly, all members of one subfamily are individually numbered (Mackenzie et al., 1997).

All UGTs transfer the activated sugar moiety from a donor onto the acceptor molecule. In plants, the donor is mostly UDP-glucose (UDP-Glc) but can also be UDP-galactose, UDP-xylose, UDPglucoronic acid or UDP-rhamnose (Vogt \& Jones, 2000). The glucose (Glc) moiety of UDP-Glc derives from Glc-1-phosphate reacts with UTP-nucleotide releasing pyrophosphate. For this reaction two UTP-Glc-1-phosphate uridylyltransferases are known in A. thaliana (Meng et al., 2009). Then, the actual UGT-reactions can take place: The UGT individual binds the co-substrate and transfers the Glc moiety from the donor onto the deprotonated group of a substrate-acceptor (Reaction 1). Usually, hydroxy groups ( $\mathrm{OH}-)$, thioles ( $\mathrm{SH}-)$, secondary amines ( $\mathrm{NH}-$ ), and rarely methines (CH-) can be used for glycosylation by UGTs (Bowles et al., 2006).

Reaction 1

$$
\mathrm{R}-\mathrm{OH}+\mathrm{UDP}-\mathrm{Glc} \stackrel{\mathrm{UGT}}{\longrightarrow} \mathrm{R}-\mathrm{O}-\mathrm{Glc}+\mathrm{UDP}
$$

R-OH: Hydroxy-substrate, sugar acceptor

UDP-Glc: Uridine diphosphate glucose, sugar-donor

UGT: Uridine diphosphate-dependent glycosyltransferase

$\mathrm{R}-\mathrm{O}$-Glc: Product, $\mathrm{O}$-glucosyl-substrate

UDP: Uridine diphosphate 
All plant UGTs that could be analyzed by x-ray crystallography so far share a common protein structure (fold-B). This structure features two domains split by a cleft. The UDP-Glc-binding motif is located at the C-terminal domain and harbors a general Rossmann-fold motif to coordinate the sugar-nucleotide (Albesa-Jové \& Guerin, 2016; Bowles et al., 2006). This Rossmann-fold motif is a general structural feature of proteins to coordinate nucleotides by an alternating order of $\beta$-sheets and $\alpha$-helices (Lesk, 1995). This C-terminal motif is highly conserved and characteristic for all UGT enzymes. In this 40-44 amino acid-long region, six characteristic amino acids achieve the direct UDP-Glc binding (Figure 1-5A) (Albesa-Jové \& Guerin, 2016; Bowles et al., 2006). This motif was identified by x-ray crystallography of the glycosyltransferase UGT78A5 from Vitis vinifera (V. vinifera, VvGT1), with the bound cofactor in the crystal structure (Figure 1-5A) (Offen et al., 2006). On the other side of the cleft, the Nterminal domain is specific for substrate binding. Thus, the general sequence homology is much lower but the motif of a catalytic histidine and an assisting aspartate is well conserved (Figure 1-5B). This motif was proposed by the x-ray crystal structure of VvGT1 and the glucosyltransferase UGT74F2 from A. thaliana (George Thompson et al., 2017; Offen et al., 2006). UGT74F2 uses SA as substrate to form specifically the SA-glucosyl ester (SA-GE), (George Thompson et al., 2017; Lim et al., 2002). Here, a tyrosine, an aspartate and the catalytic histidine coordinate SA as substrate in a way that the hydroxy group of the acid can attack the anomeric carbon of UDP-Glc by a $S_{N} 2$-reaction mechanism (see Figure 1-5B) (Albesa-Jové \& Guerin, 2016; Bowles et al., 2006). In the case of a glycosidic product formed, the assisting aspartate deprotonates the secondary amine in the imidazole ring of the catalytic histidine. Consequently, the second nitrogen of the imidazole ring, which is a tertiary amine, compensates for that by deprotonating the hydroxy group of the substrate (Albesa-Jové \& Guerin, 2016; George Thompson et al., 2017). Subsequently, the substrate anion targets the $\mathrm{C} 1$ of the sugar-donor by a nucleophilic attack. These reaction mechanisms explain the so-called inverting reaction of this class of UGTs (Lairson et al., 2008): The activated sugar donor is in the $\alpha$-configuration and nucleophilic attack of the substrate-anion from behind leads to a flip into the $\beta$-conformation of the sugar in a $S_{N} 2$-reaction mechanism. Hence, the orientation of the substrate to the catalytic histidine is crucial for catalysis. In the case of a hydroxy group, the anionic oxygen atom forms a glyosidic product with the sugar. In the case of a carboxy group, the carbanion forms a glycosyl ester (GE) as product (see Figure 1-5B). For example, the two homologue proteins UGT74F1 and UGT74F2 are characterized as SA-UGTs in A. thaliana. Thereby, UGT74F1 coordinates the SAsubstrates via the carboxy group of the benzoate and the 2-hydroxy group gets deprotonated to from a glycoside. UGT74F2, on the other side, coordinates the SA-molecule via the hydroxy group and the carboxy group gets deprotonated to form a GE (George Thompson et al., 2017). However, UGT74F1 and UGT74F2 have overlapping activates towards the respective other 
reaction (George Thompson et al., 2017; Lim et al., 2002). Generally, the catalytic sites of the UGT enzymes show high regio-selectivity and -specificity for the reactive groups, whereas, the substrate specificity tends to be broad (Vogt \& Jones, 2000).

A)

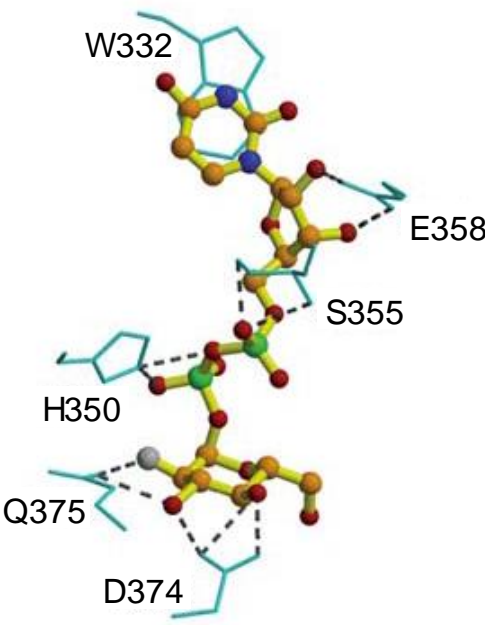

B)

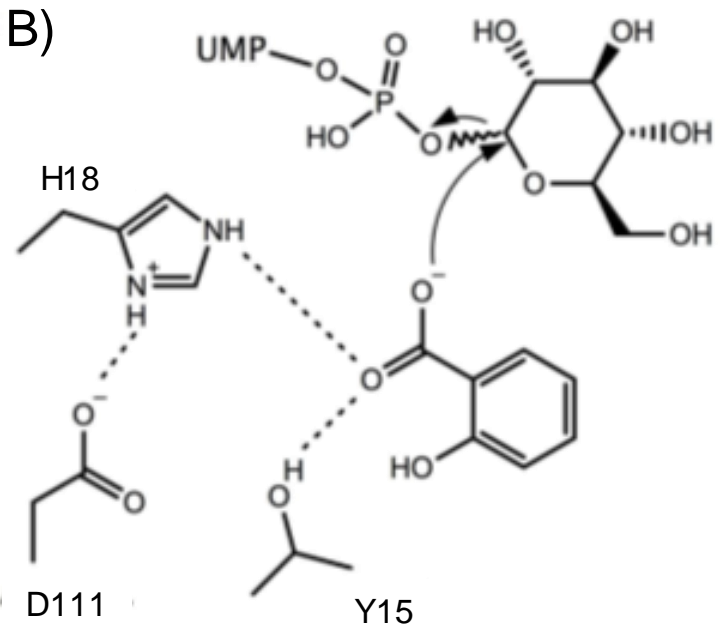

332-W APQAEVL AHE AVG AFVTHCGWNSL WESV AG GVPLICRPFFDQ-375

\section{Figure 1-5: Catalytic motifs of UDP-dependent glycosyltransferases}

UDP-dependent glycosyltransferases (UGTs) use an activated sugar donor, e g. UDP-Glc, to transfer the sugar moiety onto the substrate. Hence, all UGTs share a conserved motif for the binding of the sugar donor and the catalytic site. A) Conserved binding motif of UDP-Glc in the active site of VvGT1. The UDP-Glc orientation is achieved by six conserved amino acids (marked in the amino acid sequence by red letters) (taken from Bowles et al., 2006; Offen et al., 2006). B) The catalytic site of UGT74F2 with the coordinated substrates salicylic acid (SA) and UDP-Glc. Hydrogen bonds are given as dashed lines. The reaction mechanism is indicated with arrows: $\mathrm{Y} 15, \mathrm{H} 18$, and D111 coordinate $\mathrm{SA} ; \mathrm{S}_{\mathrm{N}} 2$ attack by the hydroxy group of the acid to the anomeric carbon of UDP-Glc results in the formation of SA-glucosyl ester (taken from George Thompson et al., 2017).

There are 120 genes encoding UGTs predicted in the A. thaliana genome. Thereof, 109 carry the specific UDP-Glc-binding motif and 107 out of these 109 share nine conserved sequence-regions (Ross et al., 2001). These UGTs were arranged in a phylogenetic tree into 14 groups depending on the amino acid similarity of the nine characteristic motifs (named A-N, Supplemental figure 1, membership > $60 \%$ similarity) (Ross et al., 2001). Although general catalytic preferences cannot be predicted, the group L (consisting of members of the UGT-subfamilies UGT74, UGT75 and UGT 84) contains UGTs, which form mainly GE-products with IAA and SA. UGT84B1, UGT74E2, and UGT74D1 are described to glycosylate auxins like indole-3-carboxylic (ICA) acid and indole-3-butyric acid (IBA) (Jackson et al., 2001; Jin et al., 2013; Tanaka et al., 2014). For IAA the glycosylation has major influence on the hormone homeostasis. In this case, $90 \%$ of all IAA is conjugated via an amide bond, $10 \%$ are linked as GE for inactivation and storage and only $1 \%$ is free IAA (Woodward \& Bartel, 2005). UGT84A1 and UGT84A3 are described to produce phenylpropanoid-GE as precursors of lignin synthesis. Here, the glycosylation is thought to promote export of the monomers for extracellular polymerization (Lim et al., 2001). In this L 
group, also UGT74F1 and UGT74F2, which glycosylate SA, are clustered (see above, (George Thompson et al., 2017; Lim et al., 2002). For 2-O-glucosyl-SA (2-O-Glc-SA), it has been reported to be localized in the vacuole (in soybean (Dean et al., 2003) and in tobacco (Dean et al., 2005)). The group H of UGTs harbors all UGT76 enzymes. This subfamily is not yet well characterized. Here, UGT76B1 is published to form a ( $2 R, 3 R)$-2-hydroxy-3-methylpentanoic acid glycoside (HMPA-Glc). The activity was found after $U G T 76 B 1$ was identified as the top induced UGT-gene after various biotic and abiotic stresses. UGT76B1 might regulate SA and JA-Ile crosstalk in plant stress response by producing HMPA-Glc

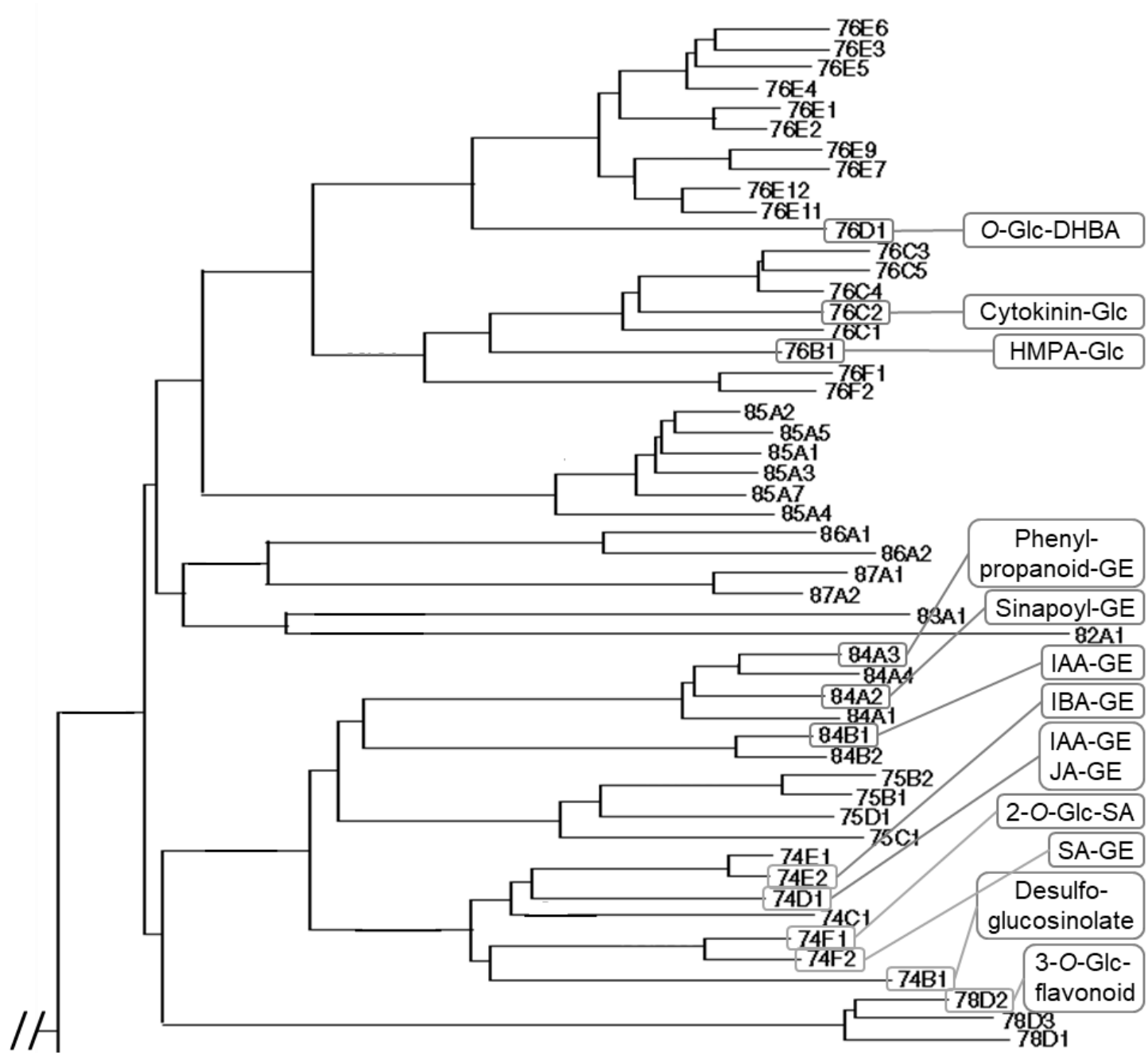

Figure 1-6: Phylogenetic tree of UGTs of $A$. thaliana related in hormone homeostasis

Phylogenetic tree of 51 out of 107 UGT genes of A. thaliana. The selected branch includes several genes (indicated by grey ellipses), whose gene products are involved in hormone homeostasis or stress response. Enzymatic products of selected UGTs are given in black boxes. The complete phylogenetic tree is shown in Supplemental figure 1. GE, glycosyl ester; HMPA-Glc, $(2 R, 3 R)$-2-hydroxy-3methylpentanoic acid glycoside; DHBA, dihydroxy-benzoic acid; IAA, indole-3-acetic acid; IBA, indole3-butylric acid; JA, jasmonic acid; 3-O-Glc-flavonoid, 3- $O$-glycosyl-flavonoid. Indicated activities are taken from Bowles et al., 2006; George Thompson et al., 2017; Hou et al., 2004; Huang et al., 2018; Jackson et al., 2001; Lim et al., 2002; Ross et al., 2001; Song, 2005; Tanaka et al., 2014; von Saint Paul et al., 2011. 
(von Saint Paul et al., 2011). In addition to that, UGT76C2 was characterized as being specific for the amine-glycosylation of cytokinins after drought stress (Hou et al., 2004). Although not much is known about the group $\mathrm{H}$, the activities that are known could point towards diverse glycosylation activities within the plant's defense response and phytohormone homeostasis.

\subsubsection{GLYCOSYLATION OF JASMONATES}

Glucose derivatives of jasmonates, like JA-glucosyl ester (JA-GE) (Miersch et al., 1987; Swiatek et al., 2004), the 12-O-Glc-JA (Miersch et al., 2008), and the 12-O-glucosyl-JA-Ile (12-O-GlcJA-Ile) (Kitaoka et al., 2014) have been identified in many plant species (Figure 1-3) (Wasternack $\&$ Feussner, 2018). Furthermore, a JA-Ile glucosyl ester has been postulated as putative natural compound (Wasternack \& Hause, 2013). Although, there is a set of glycosylated jasmonate, no intra- and intercellular functions are known for any of them (Koo, 2018). In N. tabacum cell cultures (Bright Yellow-2-cells), JA-application promotes the formation of JA-GE but also the export of 12-OH-JA (Swiatek et al., 2004). In A. thaliana, UGT74D1, named UDP-glucose:JA glucosyltransferase 1 (JGT1), was published to produce JA-GE but already that study showed higher affinities towards auxins and even dihydro-JA (Song, 2005). Anyhow, the same enzyme was found to be specific for IAA and 2-oxindole-3-acetic acid but without JA being tested as mentioned above (Jin et al., 2013; Tanaka et al., 2014). In summary, UGT74D1 seems to be rather unspecific and forms GEs of different phytohormones. This might point towards a putative function in homeostasis by balancing levels of several phytohormones.

In the case of the $\omega$-glycosylation of 12-OH-JA and 12-OH-JA-Ile, the addition of the Glc requires hydroxylation beforehand. Likewise, it follows the detoxification phases I and II (see above). Possibly, a similar inactivation mechanism could exist as described for SA in the vacuole (Dean et al., 2005; Dean et al., 2003) or possibly in the apoplast (Dietz et al., 2000).

So far, one study has detected $12-O$-Glc-JA-Ile after wounding in A. thaliana, $N$. tabacum, and Glycine max (Kitaoka et al., 2014). However, the levels were much lower than for 12-OH-JA. Nevertheless, the same group found $12-O$-Glc-JA-Ile to be de-conjugated to $12-O$-Glc-JA by IAR3 in vitro (Zhang et al., 2016). Together, there might be a physiological activity of $12-O$-GlcJA-Ile.

12-OH-JA and 12-O-Glc-JA may induce tuber formation in S. tuberosum (Yoshihara et al., 2014). Despite, 12-OH-JA and 12- $\mathrm{HSO}_{4}-\mathrm{JA}$ do not induce typical JA-responses like inhibiting root growth and germination rates in Solanum lycopersicum (S. lycopersicum) (Gidda et al., 2003; Miersch et al., 2008). All three JA-derivatives are induced by JA-Ile/COI but do not stimulate the defense-signaling themselves. Thus, it is highly unlikely that those derivatives can be metabolized back to JA (Gidda et al., 2003; Miersch et al., 2008). Nevertheless, the balance of 12-HSO $-\mathrm{JA}$ and $12-O$-Glc-JA seems to be tightly regulated. On the one hand, a ST2a over-expressing line 
shows enhanced levels of $12-\mathrm{HSO}_{4}-\mathrm{JA}$ over $12-\mathrm{O}$-Glc-JA in S. lycopersicum (Wasternack \& Hause, 2013). On the other hand, a mutant with disrupted 12- $\mathrm{HSO}_{4}-\mathrm{JA}-l e v e l s ~ s h o w s ~ e l e v a t e d ~ 12-$ $O$-Glc-JA-abundance (Mugford et al., 2009). Both reactions seem to compete for the joint substrate 12-OH-JA. In addition to this, 12-OH-JA and/or 12-O-Glc-JA are mobile compounds in S. tuberosum and N. tabacum. After application of radiolabeled 12-OH-JA, both radiolabel metabolites (12-OH-JA and 12-O-Glc-JA) could be detected in distal leaves (Seto et al., 2009). Hence, glycosylation might change or retain the localization of 12-OH-JA. On top of this, there is a $\beta$-glucosidase specifically hydrolyzing $12-O$-Glc-JA to $12-\mathrm{OH}-\mathrm{JA}$ in $\operatorname{Oryza}$ sativa $(O$. sativa) (Wakuta et al., 2010). In O. sativa and N. tabacum, there are UGTs specific for SA-glycosylation (UGT74H3 and UGT74G1-like respectively), which also tolerate 12-OH-JA (Seto et al., 2011; Seto et al., 2009). Nevertheless, no specific UGTs have been found for any of those glycosylation reactions of jasmonates yet (Koo, 2018; Wasternack \& Feussner, 2018).

Up to now, there is just one physiological effect of 12-O-Glc-JA known, which acts as a COI1JAZ independent leaf-closing factor in Samanea saman (S. saman). Noticeable, this effect is induced to a lesser extent by 12-OH-JA application, too (Nakamura et al., 2011). Therefore, specific and individual functions of 12-OH-JA and its glycoside cannot be addressed without knowing the catalytic enzymes and using the respective plant mutants. 


\section{AIMS OF THE STUDY}

The overall topic concerns the metabolic fate of jasmonates in plant defense of wounded A. thaliana. Especially the catabolic fate may be highly diverse including signal termination, degradation, storage as well as regulation and balance with other phytohormones. The initial hydroxylation of the active JA-Ile leads to inactivation (Heitz et al., 2012). On top of this, there are three different glycosylated jasmonates in A. thaliana, which change the properties of the free compounds. In such cases, a glycosylation reaction increases the solubility of jasmonates and might tag the molecules for an altered localization e.g. for deposition in the vacuole or the apoplast (Dean et al., 2005; Dean et al., 2003; Dietz et al., 2000). All these derivatives are yet without a function. Consequently, it is important to identify the specific UGTs first. Hereby, it is crucial to characterize them enzymatically in respect to their substrate specificity and selectivity. In addition, kinetic parameters will give detailed information about the UGT's preference and activity. Vogt and Jones, 2000, stress the importance of detailed enzymatic data to conclude real activities. Such findings will complete the set of catalytic enzymes acting on jasmonates (see Figure 1-3). Generally, the responsible UGT(s) will dramatically increase the understanding on the whole pathway in response to wounding, feeding insects or necrotrophic pathogens and phytohormone signal termination. Then, investigations of all reactions, their crosstalk, and regulation might address different roles of the glycosylated jasmonates.

This work follows the approach to mimic defense by mechanical wounding in the model plant A. thaliana (Koo \& Howe, 2009). After wounding, jasmonates follow a chronological sequence of metabolic modifications (Bruckhoff et al., 2016; Iven et al., 2014). This work wants to use this knowledge and postulates that also the gene expression of the responsible UGT-candidates might follow such temporal order since UGTs are usually regulated on this level (Vogt \& Jones, 2000). In this way, the stringent series of metabolic modifications of jasmonates can be achieved by exact and controlled gene activations. Once putative candidates have been identified, the UGTs will be tested with multiple experimental layers to characterize them enzymatically. On top of this, the UGT candidates will be investigated in vivo using gene editing to generate plant mutants. Likewise, it may be possible to separate the physiological functions of 12-OH-JA and 12-O-GlcJA. 


\section{Material ANd Methods}

\subsection{MATERIAL}

\subsubsection{EQUIPMENT}

Table 3-1: Equipment

\begin{tabular}{|c|c|}
\hline Equipment & Supplier \\
\hline $\begin{array}{l}\text { Acquity UPLC BEH RP } 18 \text { column }(1 \times 100 \mathrm{~mm} \text {, } \\
1.7 \mu \mathrm{m} \text { particle size })\end{array}$ & Waters Corporation (Milford, USA) \\
\hline $\begin{array}{l}\text { Acquity UPLC HSS T3 column }(1 \times 100 \mathrm{~mm}, 1.8 \\
\mu \mathrm{m} \text { particle size })\end{array}$ & Waters Corporation (Milford, USA) \\
\hline $\begin{array}{l}\text { Acquity UPLC system with (or without) UPLC } \\
\text { eLambda } 800 \mathrm{~nm} \text { PDA detector }\end{array}$ & Waters Corporation (Milford, USA) \\
\hline Agilent 1100 HPLC system & Agilent Technologies (Waldbronn, Germany) \\
\hline Agilent 1200 UHPLC system & Agilent Technologies (Waldbronn, Germany) \\
\hline ÄKTA prime plus & GE Healthcare (Little Chalfont, UK) \\
\hline ÄKTA prime system & GE Healthcare (Little Chalfont, UK) \\
\hline $\begin{array}{l}\text { Applied Biosystems } 4000 \text { quadrupole/linear ion } \\
\text { trap mass spectrometer }\end{array}$ & MDS Sciex (Framingham, USA) \\
\hline $\begin{array}{l}\text { Agilent } 6540 \text { Accurate-Mass Quadrupole-Time Of } \\
\text { Flight (Q-TOF) mass spectrometer }\end{array}$ & Agilent Technologies (Waldbronn, Germany) \\
\hline Arium pro Ultrapure Water System & Sartorius AG (Goettingen, Germany) \\
\hline Avanti J-25 centrifuge & Beckmann Coulter GmbH (Krefeld, Germany) \\
\hline Biometra BioDocAnalyzer & Analytik Jena AG (Jena, Germany) \\
\hline CanonScan 8000F scanner & Canon Incorporated (Tokyo, Japan) \\
\hline Centrifuge $5415 \mathrm{D}$ & Eppendorf AG (Hamburg, Germany) \\
\hline Centrifuge $5417 \mathrm{R}$ & Eppendorf AG (Hamburg, Germany) \\
\hline Centrifuge $5810 \mathrm{R}$ & Eppendorf AG (Hamburg, Germany) \\
\hline Climate chambers & $\begin{array}{l}\text { YORK Refrigeration, YORK Industriekaelte } \\
\text { GmbH \& Co. KG (Mannheim, Germany) }\end{array}$ \\
\hline Fluorescent image analyzer FLA-3000 & Fujifilm Corporation (Tokyo, Japan) \\
\hline HiLoad 16/600 Superdex 75 prep grade & GE Healthcare (Little Chalfont, UK) \\
\hline HiLoad 26/600 Superdex 200 prep grade & GE Healthcare (Little Chalfont, UK) \\
\hline HisTALON Superflow Cartridge, $1 \mathrm{~mL}, 5 \mathrm{~mL}$ & $\begin{array}{l}\text { Takara Bio Europe (Saint-Germain-en-Laye, } \\
\text { France) }\end{array}$ \\
\hline HisTrap HP, $1 \mathrm{~mL}, 5 \mathrm{~mL}$ & GE Healthcare (Little Chalfont, UK) \\
\hline HiTrap Desalting $5 \mathrm{~mL}$ & GE Healthcare (Little Chalfont, UK) \\
\hline $\begin{array}{l}\text { ZORBAX RX-SIL ( } 4.6 \text { x } 50 \text { mm, } 1.8 \mu \mathrm{m} \text { particle } \\
\text { size) }\end{array}$ & Agilent (Waldbronn, Germany) \\
\hline PCR detection systems iQ5 real-time & Bio-Rad Laboratories GmbH (Muenich, Germany) \\
\hline JA10 rotor & Beckman Coulter GmbH (Krefeld, Germany) \\
\hline JA25.50 rotor & Beckman Coulter GmbH (Krefeld, Germany) \\
\hline LCT Premier TOF-MS & Waters Corporation (Milford, USA) \\
\hline Mastercycler gradient & Eppendorf AG (Hamburg, Germany) \\
\hline Mastercycler personal & Eppendorf AG (Hamburg, Germany) \\
\hline Microfluidizer M-110L & Microfluidic (Westwood, USA) \\
\hline Mini-PROTEAN 3 electrophoresis system & Bio-Rad Laboratories GmbH (Munich, Germany) \\
\hline $\begin{array}{l}\text { Mixer Ball Mill MM200 with stainless steel } \\
\text { grinding jars or PTEE-jars }\end{array}$ & Retsch GmbH (Haan, Germany) \\
\hline NanoDrop 2000 spectrophotometer & Thermo Fisher Scientific (Waltham, USA) \\
\hline NGM 68 Nitrogen-Membrane-Generator & cmc Instruments (Eschborn, Germany) \\
\hline Percival CU-36L/D & Percival Scientific Inc. (Perry, USA) \\
\hline Premium Freezer & Liebherr (Bulle, Switzerland) \\
\hline Quartz SUPERSIL cuvettes & Hellma Analytics, Muellheim, Germany \\
\hline
\end{tabular}


RK 51005 Ultrasonic cleaner

Sterile bench Prettl-Telstar BioII-A

TriVersa NanoMate

Ultraflow Freezer

Ultrasonic unit Branson Sonifier Cell disruptor B15 Branson Ultrasonics Corporation (Danbury, USA)

V-630 UV/Vis Spectrophotometer
Sonorex (Moerfelden-Walldorf, Germany)

Telstar (Terrassa, Spain)

Advion (Ithaca, USA)

JASCO Corporation (Hachioji, Japan)

\subsubsection{SOFTWARE}

Table 3-2: Software

\begin{tabular}{ll}
\hline Software & Supplier \\
\hline Analyst software 1.6.2 & Applied Biosystems (Darmstadt, Germany) \\
Bildanalyseprogramm1.0.4.6 & Datinf GmbH (Tuebingen, Germany) \\
Biometra BioDocAnalyzer 2.2 & Analytik Jena AB (Jena, Germany) \\
ChemBioDraw 14.0.0.117 & PerkinElmer (Waltham, USA) \\
Geneious 8.1 & Biomatter Ltd. (Auckland, New Zealand) \\
Inkscape Vector Graphics Editor 0.92.1.1 & Inkscape Project \\
MarkerLynx for MassLynx software & Waters Corporation (Milford, USA) \\
Marvis Suite 2.6 & Kaever et al., 2014 \\
MassHunter Quantitative Analysis B.05.00 & Agilent Technologies (Santa-Clara, USA) \\
MassHunter Workstation Acquisition software & Agilent Technologies (Santa-Clara, USA) \\
B.04.00 & \\
MassLynx software 4.1 & Waters Corporation (Milford, USA) \\
Microsoft Office 2016 & Microsoft Corporation (Redmond, USA) \\
NanoDrop & Thermo Fisher Scientific (Waltham, USA) \\
Origin 8.5 & Origin Lab (Northampton, Massachusetts, USA) \\
Photoshop CS6 & Adobe Systems Incorporated (San Jose, \\
& California, USA) \\
Pymol v0.99 & DeLano Scientific LLC (San Francisco, USA) \\
iQ5 Optical System Software & Bio-Rad Laboratories GmbH (Muenich, \\
& Germany) \\
SigmaPlot & Systat Software GmbH (Erkrath, Germany) \\
Spectra Manager II Software & JASCO Corporation (Hachioji, Japan) \\
\hline
\end{tabular}

\subsubsection{CONSUMABLES}

All general consumables were purchased from Sarstedt AG \& Co. KG (Nuembrecht, Germany), if not stated otherwise.

Table 3-3: Consumables

\begin{tabular}{ll}
\hline Product & Supplier \\
\hline Glass vials for HPLC & Macherey-Nagel (Dueren, Germany) \\
Glass vials for UPLC & Macherey-Nagel (Dueren, Germany) \\
Micropore tape & 3M Science. Applied to Life (St Paul, USA) \\
Roti-NC, pore size $2 \mu \mathrm{m}$ & Carl Roth \& Co. (Karlsruhe, Germany) \\
Silica gel 60 plate & Merck KGaA, Darmstadt, Germany \\
Spin-X UF 6, 30 kDa cut-off & Corning B.V. Life Science (Amsterdam, \\
& Netherlands) \\
Soil: Fruehstofer Erde & Industrie Erdwerk Archut (Lauterbach-Wallenrod, \\
& Germany) \\
Soil: Fruehstofer Erde, T25 fein & Industrie Erdwerk Archut (Lauterbach-Wallenrod, \\
& Germany) \\
\hline
\end{tabular}




\subsubsection{CHEMICALS}

All chemicals were purchased from Carl Roth \& Co. (Karlsruhe, Germany) and Merck KGaA (Darmstadt, Germany) if not stated otherwise. Solvents like methanol, ethanol, dichlormethane, and acetonitrile (ACN) were supplied by Thermo Fisher Scientific (Waltham, USA) in high performance liquid chromatography (HPLC)- or liquid chromatography (LC) coupled to mass spectrometry (MS) (LC-MS)-grade. Ultra-pure water was taken from an Arium pro Ultrapure Water System (Sartorius, Goettingen, Germany).

Table 3-4: Chemicals

\begin{tabular}{|c|c|}
\hline Chemical & Manufacturer \\
\hline 11-Hydroxy-JA & $\begin{array}{l}\text { Kindly provided by Dr. Otto Miersch (University of } \\
\text { Halle/Wittenberg, Germany) }\end{array}$ \\
\hline 12-Hydroxy-JA methyl ester & $\begin{array}{l}\text { Kindly provided by Dr. Otto Miersch (University of } \\
\text { Halle/Wittenberg, Germany) }\end{array}$ \\
\hline 12-Hydroxy-JA & $\begin{array}{l}\text { Kindly provided by Dr. Otto Miersch (University of } \\
\text { Halle/Wittenberg, Germany) }\end{array}$ \\
\hline 12-O-Glycosyl-hydroxy-JA & $\begin{array}{l}\text { Kindly provided by Dr. Otto Miersch (University of } \\
\text { Halle/Wittenberg, Germany) }\end{array}$ \\
\hline 3-Hydroxy-hexadecanoic acid & Larodan (Solna, Sweden) \\
\hline Dihydro-kaempferol & $\begin{array}{l}\text { PhytoLab GmbH \&C. KG (Vestengergsgreuth, } \\
\text { Germany) }\end{array}$ \\
\hline Dihydro-myricetin & $\begin{array}{l}\text { PhytoLab GmbH \&C. KG (Vestengergsgreuth, } \\
\text { Germany) }\end{array}$ \\
\hline 9,12,13-Trihydroxy-octadecadienoic acid & Larodan (Solna, Sweden) \\
\hline Gibberellic acid 3 & Duchefa Biochemie B.V (Haarlem, Netherlands) \\
\hline Microagar & Duchefa Biochemie B.V (Haarlem, Netherlands) \\
\hline Silwet L-77 silicone surfactant & $\begin{array}{l}\text { Momentive Performance Materials Inc., Waterford, } \\
\text { USA }\end{array}$ \\
\hline 9,12,13-Trihydroxy-octadecaenoic acid & Larodan (Solna, Sweden) \\
\hline $\mathrm{D}_{5}$ 12-oxophytodienoic acid & $\begin{array}{l}\text { Kindly provided by Dr. Otto Miersch (University of } \\
\text { Halle/Wittenberg, Germany) }\end{array}$ \\
\hline $\mathrm{D}_{5}$ jasmonic acid & $\begin{array}{l}\text { Kindly provided by Dr. Otto Miersch (University of } \\
\text { Halle/Wittenberg, Germany) }\end{array}$ \\
\hline $\mathrm{D}_{4}$ jasmonoyl-leucine & $\begin{array}{l}\text { Kindly provided by Dr. Otto Miersch (University of } \\
\text { Halle/Wittenberg, Germany) }\end{array}$ \\
\hline $\mathrm{D}_{4}$ salicylic acid & Isotopes (Quebec, Canada) \\
\hline $\mathrm{D}_{5}$ indole-3-acetic acid & Eurisotop (Freising, Germany) \\
\hline $\mathrm{D}_{6}$ abcisic acid & Isotopes (Quebec, Canada) \\
\hline 2-oxothiazolidine-4-carboxylic acid & Merck KGaA (Darmstadt, Germany) \\
\hline Uridine diphosphate glucose [glucose $\left.{ }^{14} \mathrm{C}(\mathrm{U})\right]$ & PerkinElmer Inc. (Waltham, USA) \\
\hline
\end{tabular}




\subsubsection{ENZYMES, SIZE MARKERS, ANTIBODIES, AND MOLECULAR BIOLOGICAL KITS}

Table 3-5: Enzymes, size markers, antibodies, and molecular biological kits

\begin{tabular}{ll}
\hline Enzymes & Supplier \\
\hline DNAse I from bovine pancreas & Merck KGaA (Darmstadt, Germany) \\
Jump-start RedTaq ready mix & Merck KGaA (Darmstadt, Germany) \\
Lactatdehydrogenase & Boehringer Mannheim GmbH (Mannheim, \\
& Germany) \\
Lysozyme from chicken egg white, 70 000 U/mL & Merck KGaA (Darmstadt, Germany) \\
Phusion High-Fidelity Polymerase & Thermo Fisher Scientific (Waltham, USA \\
Pyruvatkinase & Boeringer Mannheim GmbH (Mannheim, \\
& Germany) \\
Restriction endonuclease enzymes & Thermo Fisher Scientific (Waltham, USA) \\
T4-DNA-Ligase & Thermo Fisher Scientific (Waltham, USA) \\
Takyon No ROX SYBR Mastermix blue dTTP & Kaneka Eurogentec S.A. (Seraing, Belgium) \\
\hline Size marker & \\
\hline Gel Filtration Standard & Bio-Rad Laboratories GmbH (Muenich, \\
& Germany) \\
Gene-Ruler 1kb DNA Ladder & Thermo Fisher Scientific (Waltham, USA) \\
Roti-Mark standard & Carl Roth GmbH \& Co. KG (Karlsruhe, \\
& Germany) \\
Unstained Protein Molecular Weight Marker & Thermo Fisher Scientific (Waltham, USA) \\
\hline Molecular biological kits & \\
\hline GenElute Plasmid Miniprep Kit & Merck KGaA (Darmstadt, Germany) \\
Nucleospin Gel and PCR Clean-up & Macherey-Nagel (Dueren, Germany) \\
\hline Antibodies & \\
\hline Tetra-His Antibody & Quiagen (Hilden, Germany) \\
Goat anti mouse lgG & Merck KGaA (Darmstadt, Germany) \\
\hline
\end{tabular}

\subsubsection{MEDIA}

Table 3-6: Antibiotics

Given are the final concentrations of the antibiotics.

\begin{tabular}{lc}
\hline Antibiotics & Concentration $[\mu \mathrm{g} / \mathrm{mL}]$ \\
\hline Kanamycin & 25 \\
Carbenicillin & 100 \\
Rifampicin & 50 \\
Chloramphenicol & 35 \\
Hygromycin & 50 \\
\hline
\end{tabular}

\section{Table 3-7: Media for plant cultivation}

In sterile conditions, plants were cultivated on plates with 1/2 Murashige Skoog medium. The ingredients were dissolved in water. Weight per volume, w/v.

\begin{tabular}{lr}
\hline Murashige Skoog powder & $0.22 \%[\mathrm{w} / \mathrm{v}]$ \\
sucrose & $0.05 \%[\mathrm{w} / \mathrm{v}]$ \\
Microagar & $0.2 \%[\mathrm{w} / \mathrm{v}]$ \\
\hline
\end{tabular}




\section{Table 3-8: Media for $E$. coli cultivation}

The cultures were used for DNA-amplification and cloning as well as pre-cultures and main-cultures for protein expression. E. coli were transformed with different plasmids and cultivation was always connected to antibiotic selection (Table 3-6). The standard medium for bacteria cultivation was LuriaBertani (LB) medium. Protein expression was done in special auto-induction medium (ZYP-5052) like in Studier, 2002. During preparation, ammonium and phosphate salts were added last to avoid precipitation. Volume per volume, v/v; weight per volume, w/v.

\begin{tabular}{|c|c|}
\hline \multicolumn{2}{|l|}{ LB medium } \\
\hline $\mathrm{NaCl}$ & $5 \mathrm{~g} / \mathrm{L}$ \\
\hline Peptone & $10 \mathrm{~g} / \mathrm{L}$ \\
\hline Yeast extract & $10 \mathrm{~g} / \mathrm{L}$ \\
\hline \multicolumn{2}{|l|}{ ZY medium } \\
\hline Peptone & $10 \mathrm{~g} / \mathrm{L}$ \\
\hline Yeast extract & $5 \mathrm{~g} / \mathrm{L}$ \\
\hline \multicolumn{2}{|c|}{ ZYP-5052 expression medium } \\
\hline $\mathrm{MgSO}_{4}$ & $1 \mathrm{mM}$ \\
\hline $\mathrm{FeCl}_{3}$ & $10 \mu \mathrm{M}$ \\
\hline $\mathrm{CaCl}_{2}$ & $4 \mu \mathrm{M}$ \\
\hline $\mathrm{MnCl}_{2}$ & $2 \mu \mathrm{M}$ \\
\hline $\mathrm{ZnSO}_{4}$ & $2 \mu \mathrm{M}$ \\
\hline $\mathrm{CoCl}_{2}$ & $0.4 \mu \mathrm{M}$ \\
\hline $\mathrm{CuCl}_{2}$ & $0.4 \mu \mathrm{M}$ \\
\hline $\mathrm{NiCl}_{2}$ & $0.4 \mu \mathrm{M}$ \\
\hline $\mathrm{NaMoO}_{4}$ & $0.4 \mu \mathrm{M}$ \\
\hline $\mathrm{Na}_{2} \mathrm{SeO}_{3}$ & $0.4 \mu \mathrm{M}$ \\
\hline $\mathrm{H}_{3} \mathrm{BO}_{3}$ & $0.4 \mu \mathrm{M}$ \\
\hline$\left(\mathrm{NH}_{4}\right)_{2} \mathrm{SO}_{4}$ & $25 \mathrm{mM}$ \\
\hline $\mathrm{KH}_{2} \mathrm{PO}_{4}$ & $50 \mathrm{mM}$ \\
\hline $\mathrm{Na}_{2} \mathrm{HPO}_{4}$ & $50 \mathrm{mM}$ \\
\hline Glycerol & $0.5 \%[\mathrm{v} / \mathrm{v}]$ \\
\hline Glucose & $0.05 \%[\mathrm{w} / \mathrm{v}]$ \\
\hline$\alpha$-lactose & $0.2 \%[\mathrm{w} / \mathrm{v}]$ \\
\hline ZY medium & $93 \%[\mathrm{v} / \mathrm{v}]$ \\
\hline
\end{tabular}

\subsubsection{Plasmids}

Table 3-9: Plasmids

\begin{tabular}{lll}
\hline Plasmid & Selection marker & Reference \\
\hline pCambia & Kanamycin resistance & Dr. Ellen Hornung, (University of Goettingen, \\
& BASTA resistance & Germany, (Hornung et al., 2005) \\
pET24a & Kanamycin resistance & Novagen (Schwalbach, Germany) \\
pET28a & Kanamycin resistance & Novagen (Schwalbach, Germany) \\
pJET1.2/blunt & Ampicillin resistance & Thermo Fisher Scientific (Waltham, USA) \\
pUC18-Entry & Carbenicillin resistance & Dr. Ellen Hronung, University of Goettingen, \\
& & Germany (Hornung et al., 2005) \\
pHEE401E & Kanamycin resistance / & Addgene (Cambridge, USA, Wang et al., 2014) \\
& Streptomycin resistance & \\
\hline
\end{tabular}




\subsubsection{BACTERIA STRAINS}

Table 3-10: Bacterial strains

\begin{tabular}{|c|c|c|}
\hline Strain & Genotype & Reference \\
\hline Agrobacterium tumefaciens EHA & & Dr. Ellen Hornung \\
\hline 105 & & $\begin{array}{l}\text { (University of Goettingen, } \\
\text { Germany) }\end{array}$ \\
\hline Escherichia coli Rosetta II (DE3) & $\begin{array}{l}\text { (ara-leu)76974lacX74 } 4 \text { phoA } \\
\text { PvuII phoR araD139 ahpC galE } \\
\text { galK rpsL F'[lac+ lacIq pro] } \\
\text { gor522::Tn10 trxB pRARE2 } \\
\text { (CamR, StrR, TetR) }\end{array}$ & $\begin{array}{l}\text { Novagen (Schwalbach/Ts., } \\
\text { Germany) }\end{array}$ \\
\hline Escherichia coli BL21 Star (DE3) & $\begin{array}{l}\text { F- ompT hsdSB (rB-mB-) gal dcm } \\
\text { rne } 131 \text { (DE3) }\end{array}$ & $\begin{array}{l}\text { Thermo Fisher Scientific } \\
\text { (Waltham, USA) }\end{array}$ \\
\hline Escherichia coli $\mathrm{DH} 5 \alpha$ & $\begin{array}{l}\text { fhuA2 lac(del)U169 phoA glnV44 } \\
\text { Ф80' lacZ(del)M15 gyrA96 recAl } \\
\text { relA1 endA1 thi-1 hsdR17 }\end{array}$ & $\begin{array}{l}\text { New England Biolabs Ltb } \\
\text { (Ontario, Canada) }\end{array}$ \\
\hline Escherichia coli XL-1-blue & $\begin{array}{l}\text { recAlendAlgyrA96 thi-1hsdR17 } \\
\text { supE44 relA1lac[F'proAB } \\
\text { lac } 9 z M 15 \text { Tn19(Tetr)] }\end{array}$ & $\begin{array}{l}\text { Agilent Technologies } \\
\text { (Santa-Clara, USA) }\end{array}$ \\
\hline
\end{tabular}

\subsubsection{Plant Lines}

All plant lines used in this work are A. thaliana plants of the accession Col-0.

Table 3-11: Plant lines

\begin{tabular}{llll}
\hline $\begin{array}{l}\text { Locus of } \\
\text { mutation }\end{array}$ & Plant & Plant line & Reference \\
\hline At5g42650 & $\begin{array}{l}\text { Col-0 } \\
\text { delayed-dehiscence 2- } \\
2 \text { (dde2-2) }\end{array}$ & N65993 & $\begin{array}{l}\text { Nottingham Arabidopsis Stock Centre } \\
\text { Dr. Michael Stumpe (University of } \\
\text { Goettingen, Germany, von Malek } \text { et al, } \\
\text { 2002) }\end{array}$ \\
\hline
\end{tabular}

\subsubsection{OLIGONUCLEOTIDES}

Table 3-12: Oligonucleotides

\begin{tabular}{|c|c|c|c|c|}
\hline No. & Name & Sequence & Item & $\begin{array}{l}\text { rest. } \\
\text { site }\end{array}$ \\
\hline P005 & UGTE12-fwd-pET28 & ACGCATATGATGCAGGTTTTGGGAATGGAGG & Cloning & NdeI \\
\hline P006 & UGTE12-rev-pET28 & ACGCTCGAGTCATAGAGTCCTTATGAAGTGTAC & Cloning & XhoI \\
\hline P007 & UGTE1-fwd-pET28 & ACGCATATGATGGAAGAACTAGGAGTGAAGAGAAG & Cloning & NdeI \\
\hline P008 & UGTE1-rev-pET28 & ACGCTCGAGGTGAACAATGATTTTGTCTATAAATGC & Cloning & XhoI \\
\hline P026 & pJET fwd & CGACTCACTATAGGGAGAGCGGC & Sequencing & \\
\hline P027 & pJET rev & AAGAACATCGATTTTCCATGGCAG & Sequencing & \\
\hline P028 & T7-for & TAATACGACTCACTATAGGG & Sequencing & \\
\hline P029 & T7-rev & GCTAGTTATTGCTCAGCGG & Sequencing & \\
\hline P044 & pJet for outer & ACCATATCCATCCGGCGTAA & Sequencing & \\
\hline P045 & pJet rev outer & AAGAAAACCCACGCCACCTA & Sequencing & \\
\hline P129 & E1-q-left & TGCCAACTTCAGCATTTGGG & qPCR & \\
\hline P130 & E1-q-right & ACCATGCCAAAGATGAGCTC & qPCR & \\
\hline P131 & E12-q-left & TCGTCACCATTCCAGAAAGC & qPCR & \\
\hline
\end{tabular}




\begin{tabular}{|c|c|c|c|c|}
\hline P132 & E12-q-right & TTGTTGCAGCACCAACTGAC & qPCR & \\
\hline P133 & Actin8-RT_for & GGTTTTCCCCAGTGTTGTTG & qPCR & \\
\hline P134 & Actin8-RT_rev & CTCCATGTCATCCCAGTTGC & qPCR & \\
\hline P135 & UBQ5-RT_fwd & GACGCTTCATCTCGTCC & qPCR & \\
\hline P136 & UBQ5-RT_rev & GTAAACGTAGGTGAGTCCA & qPCR & \\
\hline P137 & E2-fwd & ACGCATATGGAGGAAAAGCAAG & Cloning & NdeI \\
\hline P138 & E2-rev & ACGCTCGAGCATGGAATTAAC & Cloning & XhoI \\
\hline P139 & E11-fwd & ACGCATATGGAGGAAAAGCC & Cloning & NdeI \\
\hline P140 & E11-rev & ACGCTCGAGTAGAGTCCTCATG & $\begin{array}{l}\text { Cloning } \\
\text { CRISPR- }\end{array}$ & XhoI \\
\hline P157 & U6-29p-F & TTAATCCAAACTACTGCAGCCTGAC & $\begin{array}{l}\text { Sequencing } \\
\text { CRISPR- }\end{array}$ & \\
\hline P158 & U6-29-p-R & AGCCCTCTTCTTTCGATCCATCAAC & $\begin{array}{l}\text { Sequencing } \\
\text { CRISPR- }\end{array}$ & \\
\hline P159 & U6-1t-F & GCTAAGACAAAGTGATTGGTCCGTT & $\begin{array}{l}\text { Sequencing } \\
\text { CRISPR- }\end{array}$ & \\
\hline P160 & U6-1t-R & AACGGACCAATCACTTTGTCTTAGC & Sequencing & \\
\hline P161 & A-DT1-BsF & ATATATGGTCTCGATTGCGGGAAGGCTCTTTACTCCAGTT & CRISPR/Cas9 & \\
\hline P162 & A-DT1-F0 & TGCGGGAAGGCTCTTTACTCCAGTTTTAGAGCTAGAAATAGC & CRISPR/Cas9 & \\
\hline P163 & A-DT0-BsR2 & ATATTATTGGTCTCAATCTCTTAGTCGACTCTACCAAT & CRISPR/Cas9 & \\
\hline P164 & A-DT2-BsF2 & $\begin{array}{l}\text { ATALIATIGGICICAAGATIGCAGACTCAGITAAGCTGCCIG } \\
\text { TT }\end{array}$ & CRISPR/Cas9 & \\
\hline P165 & A-DT2-F0 & TGCAGACTCAGTTAAGCTGCCTGTTTTAGAGCTAGAAATAGC & CRISPR/Cas9 & \\
\hline P166 & A-DT0-BsR3 & ATATTATTGGTCTCATCACTACTTCGTCTCTAACCAT & CRISPR/Cas9 & \\
\hline P167 & A-DT3-BsF3 & $\begin{array}{l}\text { ATATTATTGGTCTCAGTGATTGCTCTGGTAAGCTTTCTGGAA } \\
\text { GTT }\end{array}$ & CRISPR/Cas9 & \\
\hline P168 & A-DT3-F0 & TGCTCTGGTAAGCTTTCTGGAAGTTTTAGAGCTAGAAATAGC & CRISPR/Cas9 & \\
\hline P169 & A-DT4-R0 & $\begin{array}{l}\text { AACAACAGTCCTTGAAGCTCACCAATCACTACTTCGACTCTA } \\
\text { GCTGTAT }\end{array}$ & CRISPR/Cas9 & \\
\hline P170 & A-DT4-BsR & ATTATTGGTCTCTAAACAACAGTCCTTGAAGCTCAC & CRISPR/Cas9 & \\
\hline P171 & B-DT1-BsF & ATATATGGTCTCGATTGAACAGTGATGGAGAAGCCCTGTT & CRISPR/Cas9 & \\
\hline P172 & B-DT1-F0 & TGAACAGTGATGGAGAAGCCCTGTTTTAGAGCTAGAAATAGC & CRISPR/Cas9 & \\
\hline P173 & B-DT0-BsR2 & ATATTATTGGTCTCAATCTCTTAGTCGACTCTACCAAT & CRISPR/Cas9 & \\
\hline P174 & B-DT2-BsF2 & $\begin{array}{l}\text { ATATTATTGGTCTCAAGATTGTCGTGCTAAAGACGACACTGT } \\
\text { T }\end{array}$ & CRISPR/Cas 9 & \\
\hline P175 & B-DT2-F0 & TGTCGTGCTAAAGACGACACTGTTTTAGAGCTAGAAATAGC & CRISPR/Cas9 & \\
\hline P176 & B-DT0-BsR3 & ATATTATTGGTCTCATCACTACTTCGTCTCTAACCAT & CRISPR/Cas9 & \\
\hline P177 & B-DT3-BsF3 & $\begin{array}{l}\text { ATATTATTGGTCTCAGTGATTGTTTCAGCACCACAAGTGCCA } \\
\text { GTT }\end{array}$ & CRISPR/Cas9 & \\
\hline P178 & B-DT3-F0 & TGTTTCAGCACCACAAGTGCCAGTTTTAGAGCTAGAAATAGC & CRISPR/Cas9 & \\
\hline P179 & B-DT4-R0 & $\begin{array}{l}\text { AACTTCCAGAAAGCTTACCAGAGCAATCACTACTTCGACTCT } \\
\text { AGCTGTAT }\end{array}$ & CRISPR/Cas9 & \\
\hline P180 & B-DT4-BsR & ATTATTGGTCTCTAAACTTCCAGAAAGCTTACCAGAG & CRISPR/Cas9 & \\
\hline P183 & SGT-fwd & ACGGAATTCGAGAAGATGAGAGG & Cloning & EcoRI \\
\hline P184 & SGT_rev & ACGGGATCCTTTGATTTGAATTTTTG & Cloning & BamHI \\
\hline P187 & SGT_rev_new & ACGCTCGAGTCATTTGATTTGAATTT & Cloning & XhoI \\
\hline P188 & 2A-DT1-BsF & ATATATGGTCTCGATTGCGGGAAGGCTCTTTACTCCAGTT & CRISPR/Cas9 & \\
\hline P189 & 2A-DT1-F0 & $\begin{array}{l}\text { TGCGGGAAGGCTCTTTACTCCAGTTTTAGAGCTAGAAATAGC } \\
\text { AACAGGCAGCTTAACTGAGTCTGCAATCTCTTAGTCGACTCT }\end{array}$ & CRISPR/Cas9 & \\
\hline P190 & 2A-DT2-R0 & $\mathrm{AC}$ & CRISPR/Cas9 & \\
\hline P191 & 2A-DT2-BsR & АTTATTGGTCTCGAAACAGGCAGCTTAACTGAGTCTGCAA & CRISPR/Cas9 & \\
\hline P192 & 2B-DT1-BsF & ATATATGGTCTCGATTGAGGGCTTCTCCATCACTGTTGTT & CRISPR/Cas9 & \\
\hline P193 & 2B-DT1-F0 & TGAGGGCTTCTCCATCACTGTTGTTTTAGAGCTAGAAATAGC & CRISPR/Cas 9 & \\
\hline P194 & 2B-DT2-R0 & IIAGCACGACLAATLICIIS & CRISPR/Cas9 & \\
\hline P195 & 2B-DT2-BsR & ATTATTGGTCTCGAAACAGTGTCGTCTTTAGCACGACCAA & CRISPR/Cas9 & \\
\hline P237 & E1-detect (rev) & CGGTCAAGCTGCCTGGG & Seq-CRISPR & \\
\hline $\mathrm{P} 238$ & E2-detect (rev) & CAGGATCTTTCATGTCG & Seq-CRISPR & \\
\hline
\end{tabular}




\begin{tabular}{|c|c|c|c|}
\hline P239 & E11-detect (rev) & CGTTTTGTTGTCCTTTGGG & Seq-CRISPR \\
\hline $\mathrm{P} 240$ & E12-detect (rev) & CTTTGGCTGCAGCTTCAGC & Seq-CRISPR \\
\hline P241 & E2-q-left & TTGGGCCAATAGAGAGTACGC & qPCR \\
\hline P242 & E2-q-right & ACCGGAACTTGCAGTTGTTG & qPCR \\
\hline P243 & E11-q-left & TTTTGGAGCCATTGCGGATG & qPCR \\
\hline P244 & E11-q-right & ATCACTGGAAAACGGCTTGC & qPCR \\
\hline P245 & SGT-q-left & AAACCTTCGGCTCCAAAACC & qPCR \\
\hline P246 & SGT-q-right & ATCCATTGCAAGGTCAAGCG & qPCR \\
\hline P247 & E2-q-left_new & GAGCTCATCTTTGGCAAGGTTG & qPCR \\
\hline $\mathrm{P} 248$ & E2-q-right_new & TTCAACCACTCAACGCAACTC & qPCR \\
\hline
\end{tabular}

\subsection{METHODS}

The plant work dealing with A. thaliana was generally performed according to Weigel \& Glazebrook, 2002.

\subsubsection{Plant GROWTH AND TREATMENT}

\subsubsection{Plant GROWTH AND CULTIVATION}

Plants were grown either on soil or on plates in sterile conditions.

For standard growth conditions, plants were grown on soil (Fruehstofer Erde, Industrie Erdwerk Archut, Lauterbach-Wallenrod, Germany). Therefore, the seeds were sowed on steamed soil $\left(80{ }^{\circ} \mathrm{C}, 8\right.$ hours (h)) and stored at $4{ }^{\circ} \mathrm{C}$ in the dark for 2 days (d) to guarantee a synchronized germination. Then, the plants were transferred either into climate chambers with long day conditions ( $16 \mathrm{~h} \mathrm{light} \mathrm{/} 8 \mathrm{~h}$ dark, $22{ }^{\circ} \mathrm{C}, 60 \%$ humidity, and light intensity of $100 \mu \mathrm{mol} \mathrm{m}^{-2} \mathrm{~s}^{-1}$ ) for seed propagation and homozygosity tests or into Percival CU-36L/D (Percival Scientific Inc., Perry, USA) with short day conditions ( $8 \mathrm{~h}$ light $/ 16 \mathrm{~h}$ dark, $22{ }^{\circ} \mathrm{C}, 60 \%$ humidity, and light intensity of $100 \mu \mathrm{mol} \mathrm{m} \mathrm{m}^{-2}$ ) for wounding experiments (see 3.2.1.3).

For sterile growth conditions, the seeds were sterilized with bleach first. Approximately (appr.) 200 seeds were treated with $1 \mathrm{~mL}$ sterilization solution (66\% Na-hypochlorite [12\%], $0.1 \%$ Tween 20 in water) and incubated for $10 \mathrm{~min}$. Following, the solution was discarded and the seeds were washed with $0.5 \mathrm{~mL}$ pure ethanol five times and air dried. These seeds were brought onto 1/2 Murashige Skoog plates (Table 3-7). Therefore, the seeds were given in sterile $0.1 \%$ Agarose in water [weight per volume (w/v)] and applied onto the plates. The suspension was allowed to dry for appr. $1 \mathrm{~h}$ under the sterile bench. Then, the covered plate was sealed with Micropore-tape (3M Science, St Paul, USA). The plates were transferred to long day conditions ( $16 \mathrm{~h}$ light $/ 8 \mathrm{~h}$ dark, $\left.22^{\circ} \mathrm{C}\right)$. 


\subsubsection{SEED PROPAGATION FROM JA-DEFICIENT PLANTS BY JA METHYL ESTER TREATMENT}

JA-deficient plants tend to be male sterile (Caldelari et al., 2011; Stintzi \& Browse, 2000; von Malek et al., 2002). This causes problems in propagating seeds because such mutants are impaired in elongating the anthers in the flowers and, hence, not able to self-fertilize. To propagate the JAdeficient mutant dde2-2 (Table 3-11) (von Malek et al., 2002), plants were grown under long-day conditions and the flowers were sprayed every day with a solution of $0.01 \%$ JA-ME, $95 \%$, Merck KGaA, Darmstadt, Germany) in $0.1 \%$ Tween 20.

\subsubsection{WOUNDING OF ARABIDOPSIS}

Plant were wounded like described in (Mosblech, 2010). For the wounding experiment, five plants per pot $(9 \times 9 \times 8 \mathrm{~cm})$ were grown under short day conditions $\left(8 \mathrm{~h}\right.$ light $/ 16 \mathrm{~h}$ dark, $\left.22{ }^{\circ} \mathrm{C}\right)$ for six weeks. Leaves were wounded three times across the mid vein by squeezing with forceps. In this work, non-arresting Kocher's forceps were used controlled wounding of the leaves. Damaged rosette leaves were harvested by cutting the aboveground part of the plants. Usual harvesting time points were 1, 2, and 5 hpw. Control plants were harvested without of wounding. All harvested rosettes were immediately flash-frozen in liquid $\mathrm{N}_{2} .10$ plants per time point were pooled as one replicate. The plant material was stored at $-80{ }^{\circ} \mathrm{C}$ for further analyses.

\subsubsection{MOLECULAR BIOLOGICAL METHODS}

If not mentioned otherwise, all molecular biological methods were performed according to Ausubel et al., 1993, or Sambrook et al., 1989.

\subsubsection{EXTRACTION OF GENOMIC PLANT DNA}

Genomic DNA was extracted from homogenized plant material following the method of Doyle \& Doyle, 1987. It was used to amplify specific gene fragments for genotyping of plants and to detect single nucleotide polymorphisms. Therefore, appr. $100 \mathrm{mg}$ of the sample were homogenized in $250 \mu \mathrm{L}$ cetyltrimethylammonium bromide (CTAB) solution (Table 3-13) and incubated at $65{ }^{\circ} \mathrm{C}$ for 15 minutes (min). Then, $250 \mu \mathrm{L}$ of chloroform : isoamyl alcohol [24:1, volume per volume $(\mathrm{v} / \mathrm{v})]$ were added and mixed. After centrifugation at $7500 \mathrm{xg}$ at room temperature for $4 \mathrm{~min}, 180 \mu \mathrm{L}$ of the aqueous phase were taken and mixed with the same volume of isopropanol. After $2 \mathrm{~min}$ of incubation, the sample was centrifuged at $20000 \mathrm{xg}$ at room temperature for $10 \mathrm{~min}$. The supernatant was carefully removed and the pellet was washed with $75 \%$ ethanol and dried at $65^{\circ} \mathrm{C}$. Finally, the dry pellet was dissolved in $70 \mu \mathrm{L} \mathrm{H}_{2} \mathrm{O}$. 
Table 3-13: Cetyltrimethylammonium bromide solution

Cetyltrimethylammonium bromide (CTAB) extraction solution was used for extracting genomic DNA from plant tissue. Ethylenediaminetetraacetic acid (EDTA). Weight per volume, w/v.

\begin{tabular}{|c|c|}
\hline $2 \%[\mathrm{w} / \mathrm{v}]$ & CTAB \\
\hline $100 \mathrm{mM}$ & Tris/HCl pH 8.0 \\
\hline $20 \mathrm{mM}$ & EDTA \\
\hline $1.4 \mathrm{M}$ & $\mathrm{NaCl}$ \\
\hline
\end{tabular}

\subsubsection{EXTRACTION OF TOTAL PLANT RNA}

RNA from A. thaliana was isolated to amplify coding sequences of processed gene products and to monitor the expression of Arabidopsis genes quantitatively. To isolate total RNA from vegetative tissue, a modified protocol from (Onate-Sanchez \& Vicente-Carbajosa, 2008) was used. $300 \mu 1$ of extraction buffer (Table 3-14) were pipetted in a $1.5 \mathrm{~mL}$ reaction tube and $100 \mathrm{mg}$ frozen tissue-powder were weighted into the tube. The suspension was mixed roughly and incubated for $5 \mathrm{~min}$ at room temperature. Then, $100 \mu \mathrm{L}$ of protein precipitation solution (Table 3-14) were added and the suspension was incubated at $4{ }^{\circ} \mathrm{C}$ for at least $10 \mathrm{~min}$. Following, the suspensions was centrifuged at $20000 \mathrm{xg}$ and $4{ }^{\circ} \mathrm{C}$ for $10 \mathrm{~min}$. The supernatant was transferred to a fresh tube and mixed with $300 \mu \mathrm{L}$ isopropanol. This volume was centrifuged at $20000 \mathrm{xg}$ and $4{ }^{\circ} \mathrm{C}$ for $4 \mathrm{~min}$ to precipitate poly-nucleotides. Then, the supernatant was carefully removed and the pellet was washed with $500 \mu \mathrm{L} 70 \%$ ethanol and centrifuged as before. The supernatant was taken again and the pellet was dried at $50{ }^{\circ} \mathrm{C}$. The dry residue was resuspended in $20 \mu \mathrm{L}$ diethyl pyrocarbonate DEPC-water (Table 3-14). The entire volume was used for DNase I treatment. Therefore, $3 \mu \mathrm{L}$ of 10x DNase buffer were mixed with the RNA and $2 \mu \mathrm{L}$ ( 2 units) of DNase I (Thermo Fisher Scientific, Waltham, USA) were added and incubated at $37^{\circ} \mathrm{C}$ for $30 \mathrm{~min}$. The reaction was stopped by adding $2 \mu \mathrm{L} 50 \mathrm{mM}$ ethylenediaminetetraacetic acid (EDTA) and incubating at $65^{\circ} \mathrm{C}$ for $10 \mathrm{~min} .70 \mu \mathrm{L}$ of DEPC-water were added to the $30 \mu \mathrm{L}$ of DNase-treated RNA and this was mixed with $50 \mu \mathrm{L} 7.5 \mathrm{M} \mathrm{NH}_{4} \mathrm{Ac}$ and $400 \mu \mathrm{L} 100 \%$ ethanol. This solution was centrifuged at

Table 3-14: Solutions for RNA extraction

Diethyl pyrocarbonate (DEPC), ethylenediaminetetraacetic acid (EDTA), sodium dodecyl sulfate (SDS).

\begin{tabular}{cl}
\hline Cell lysis solution & \\
\hline $2 \%$ & SDS \\
$68 \mathrm{mM}$ & Sodium citrate \\
$138 \mathrm{mM}$ & Citric acid \\
$1 \mathrm{mM}$ & EDTA \\
\hline \multicolumn{2}{c}{ Protein-DNA precipitation solution } \\
\hline $4 \mathrm{M}$ & NaCl \\
$16 \mathrm{mM}$ & Sodium citrate \\
$32 \mathrm{mM}$ & Citric acid \\
\hline DEPC-water & \\
\hline $0.1 \%$ & DEPC in water dissolved $2 \mathrm{~h}$ at 37 \\
\hline
\end{tabular}


20 000x g and $4{ }^{\circ} \mathrm{C}$ for 20 min to separate RNA from degraded DNA. Afterwards, the pellet was washed with $200 \mu \mathrm{L}$ ethanol at $20000 \mathrm{x}$ g and $4{ }^{\circ} \mathrm{C}$. The supernatant was removed and the pellet was dried at $50{ }^{\circ} \mathrm{C}$. Finally, the residue was resuspended in $20 \mu \mathrm{L}$ water. The extracted RNA was stored at $-80{ }^{\circ} \mathrm{C}$.

\subsubsection{SYNTHESIS OF COMPLEMENTARY DNA}

To check the expression of genes or to clone correctly processed gene products for protein expression, RNA has to be used as template. While, especially for polymerase chain reaction (PCR), it is necessary to use DNA. Therefore, complementary DNA (cDNA) has to be synthesized from the RNA.

$1 \mu \mathrm{g}$ RNA was reversely transcribed into cDNA. Initially, the RNA was incubated with $1 \mu \mathrm{L}$ $0.5 \mu \mathrm{g} / \mu \mathrm{L}$ olido $\mathrm{dT}_{18}$-primer at $65{ }^{\circ} \mathrm{C}$ for $10 \mathrm{~min}$. Then, $2 \mu \mathrm{L} 5 \mathrm{x}$ buffer for the reverse transcriptase, $2 \mu \mathrm{L} 10 \mathrm{mM}$ deoxyribonucleoside triphosphates (dNTP) mix, and $1 \mu \mathrm{L}$ (200 units) reverse transcriptase (RevertAid, Thermo Fisher Scientific, Waltham, USA) were added to a final volume of $10 \mu \mathrm{L}$. The transcription reaction was carried out in a thermo cycler (Eppendorf cycler, Hamburg, Germany) at $37^{\circ} \mathrm{C}$ for $1 \mathrm{~h}$ and at $70{ }^{\circ} \mathrm{C}$ for $10 \mathrm{~min}$. For further use, $90 \mu \mathrm{L}$ water were added to obtain cDNA-concentrations of $300-500 \mathrm{ng} / \mu \mathrm{L}$.

\subsubsection{CULTIVATION OF E. COLI BACTERIA}

Bacteria were cultivated either in liquid culture or on solid medium. A cultivation was always connected with a selection against specific antibiotics (Table 3-9). The first selection after ligation usually was performed on plates (LB-medium (Table 3-8) with $1.5 \%$ [w/v] agar, (Duchefa Biochemie B.V, Haarlem, Netherlands)). Incubation at $37^{\circ} \mathrm{C}$ overnight allowed positively transformed cells to form single colonies, which were investigated further.

For plasmid amplification, $5 \mathrm{~mL}$ liquid LB medium containing the respective antibiotic (Table 39) were inoculated with one single colony of a plate-culture and incubated at $37{ }^{\circ} \mathrm{C}$ and 200 rotations per minute (rpm) overnight.

In preparation of protein expression, the expression cells, the vector, and the expression medium were optimized. Best yields were obtained from heterologous protein expression with the pET28a vector in BL21 Star (DE3) cells in ZYP-5052 by using auto-induction medium (Table 3-8) (Studier, 2005). This kind of medium automatically induced protein expression at low glucose and high lactose conditions without further stimulation. Firstly, $50 \mathrm{~mL}$ pre-culture were inoculated with several colonies from a cultivation plate or with the $0.5 \mathrm{~mL}$ of a glycerol stock (an aliquot of an earlier pre-culture with $30 \%$ glycerol $[\mathrm{v} / \mathrm{v}]$, stored at $-80{ }^{\circ} \mathrm{C}$ ) and cultivated in LB medium at $37^{\circ} \mathrm{C}$ overnight. Thereof, a main expression culture was prepared. Therefore, 
$250 \mathrm{~mL}$ liquid expression medium (Table 3-8) in a $1 \mathrm{~L}$ flask were inoculated with pre-culture to an optical density at $600 \mathrm{~nm}\left(\mathrm{OD}_{600}\right)$ of 0.1 and the cultures were incubated at $16{ }^{\circ} \mathrm{C}$ for $3 \mathrm{~d}$. After protein expression, the cells were harvested by centrifugation at $4000 \mathrm{xg}$ and $4{ }^{\circ} \mathrm{C}$ for $20 \mathrm{~min}$. The pellets were transferred into $50 \mathrm{~mL}$ tubes and flash-frozen in liquid $\mathrm{N}_{2}$ for storage at $-80{ }^{\circ} \mathrm{C}$.

\subsubsection{CLONING OF CODING SEQUENCES FROM A. THALIANA INTO RECOMBINANT PLASMIDS}

\subsubsection{AMPLIFICATION OF DNA FRAGMENTS USING PCR}

PCR is the standard method to amplify DNA fragments from a template sequence. The general method consists of three steps: (i) a DNA double strand is melted into single strands at a temperature of $94-98{ }^{\circ} \mathrm{C}$. (ii) Specific primers bind to the complement sequence of the single stranded template DNA. This works best at a temperature appr. $3{ }^{\circ} \mathrm{C}$ under the specific melting temperature of the primers. (iii) The primer sequences are elongated by the DNA-polymerase at its optimal temperature. These three steps are repeated for 20 - 40 circles. Thereby, the sequence of interest flanked by the two primers is multiplied exponentially. Here, two polymerase enzymes were used: Jump-start RedTaq (Merck KGaA, Darmstadt, Germany) and Phusion High-fidelity polymerase (Thermo Fisher Scientific, Waltham, USA). Both enzymes were used following the manufacturers protocol for reactions mixtures and PCR settings. All PCRs were carried out in thermo cyclers (Eppendorf cyclers, Hamburg, Germany).

Noticeably, the coding sequence of UGT74F1 was amplified from root-RNA to express the protein heterologously in bacteria (see 3.2.2.4). Interestingly, the obtained PCR product corresponds to gene product At2g43840.1 on The Arabidopsis Information Research. This differs in three base pairs (bp) from the variant At2g43840.2: at position $625 \mathrm{bp}$ from the start, the amplified sequence is adenine-adenine-guanine in variant 1 compared to tyrosine-tyrosineadenine in variant 2 . This position corresponds to exon-intron border of exon 1 and intron 1 . This differences change the amino acid sequence of variant 1 to 2 as following: valine 209 to glutamic acid and lysine 210 to glutamic acid.

\subsubsection{SEPARATION OF NUCLEOTIDES BY ELECTROPHORESIS}

Polynucleotides carry negative charges on their sugar-phosphate backbones, which are proportional to the length of the nucleotide sequence. Thus the molecules can be separated according to their size along an electrical field in a gel matrix retarding DNA movement. Here, electrophoretic separation of nucleotides was performed in horizontal $1 \%$ agarose gels in Tris/acetic acid/EDTA (TAE) buffer (Table 3-15). In case of separating RedTaq-PCR products, no additional dye was added since the RedTaq buffer already includes a dye. Other DNA samples were mixed with $1 / 6$ volume loading dye (Table 3-15). For size calibration of the nucleotide 
fragments, $5 \mu \mathrm{L}$ of the Gene-Ruler $1 \mathrm{~kb}$ DNA Ladder (Thermo Fisher Scientific, Waltham, USA) were added to the gel. After running the electrophoresis, the DNA was stained in $2 \mu \mathrm{g} / \mathrm{mL}$ ethidium bromide in TAE buffer (Table 3-15) for appr. $10 \mathrm{~min}$. DNA signals were visualized by exiting with UV light in a Biometra BioDocAnalyzer (Analytik Jena AG, Jena, Germany).

\section{Table 3-15: Buffer system for agarose gel electrophoresis}

Agarose gel electrophoresis was used to separate DNA- or RNA-fragments by size. DNA was separated in $1 \%$ agarose gels in Tris/acetic acid/ethylenediaminetetraacetic acid (EDTA) (TAE) buffer. If necessary, the DNA samples were prepared with $6 x$ loading dye. Volume per volume, $v / v$; weight per volume, $\mathrm{w} / \mathrm{v}$.

\begin{tabular}{cl}
\hline TAE buffer & \\
\hline $40 \mathrm{mM}$ & Tris \\
$20 \mathrm{mM}$ & Acetic acid \\
$2 \mathrm{mM}$ & EDTA \\
\hline 6x loading dye & \\
\hline $40 \mathrm{mM}$ & Tris \\
$2 \mathrm{mM}$ & EDTA \\
$50 \%[\mathrm{v} / \mathrm{v}]$ & Glycerol \\
$0.4 \%[\mathrm{w} / \mathrm{v}]$ & Bromphenole blue \\
\hline
\end{tabular}

\subsubsection{PURIFICATION OF Plasmids AND DNA Fragments}

Isolation of pure plasmids was performed using the GenElute Plasmid Miniprep Kit (Merck KGaA, Darmstadt, Germany) according to the protocol provided by the manufacturer. For purification of DNA fragments, the DNA solutions were separated by agarose electrophoresis (3.2.3.2) first. These separated DNA fragments were cut from the gel and purified using the NucleoSpinExtract II Kit (Macherey-Nagel, Dueren, Germany) according to the protocols provided by the manufacturer.

\subsubsection{RESTRICTION AND LIGATION OF DNA}

Specific restriction of double-stranded DNA enables site-directed cloning. The restriction sites are normally added during PCR from the primers sequences. These specific sites are cut by endonuclease restriction enzymes (Thermo Fisher Scientific, Waltham, USA) leaving complementary overhangs which can be ligated again. Originally, these enzymes defend bacteria against attacking phages by cutting foreign DNA. Commercially used endonucleases type II recognize specific palindromic DNA sequences and cut them. Type I enzymes cut next to the recognition site. In this work, digestion reactions of double stranded DNA were performed in reaction volumes of $20-50 \mu \mathrm{L}$ at $37^{\circ} \mathrm{C}$ for $1 \mathrm{~h}$ up to overnight. Reaction solutions were prepared as recommended by the manufacturer. Digested fragments were separated by agarose gel electrophoresis (3.2.3.2) and analyzed by UV light. 
DNA ligation was performed with at least two double stranded DNA fragments with complementary overhanging sequences to gain one functional DNA plasmid. The ligation reaction was performed in $10 \mu \mathrm{L}$ volume following the manufacturers concentrations for $\mathrm{T} 4$ Ligase and buffers (Thermo Fisher Scientific, Waltham, USA) and incubated for 30 min at room temperature. Concerning DNA concentrations, it is important to mix the fragments in optimal amounts. The concentration of open ends of the smaller insert is best five times higher than that of the bigger fragment, the vector (Equation 1). Nucleotide concentrations were determined in a NanoDrop spectrophotometer (Thermo Fischer Scientific, Waltham, USA) at $260 \mathrm{~nm}$. Following ligation, the reaction solutions were subsequently transformed into competent cells optimized for plasmid-amplification (Table 3-10).

Equation 1

$$
\text { Volume }(\text { insert })=\frac{5 \cdot \operatorname{size}(\text { insert }) \cdot \mathrm{c}(\text { vector }) \cdot \text { Volume }(\text { vector })}{\operatorname{size}(\text { vector }) \cdot c(\text { insert })}
$$

Volume (insert): Volume of the insert fragment for an effective ligation

Size (insert): Size of the insert in base pairs

$\mathrm{c}$ (insert): Concentration of the insert

Volume (vector): Volume of the vector fragment

Size (vector): Size of the vector in base pairs

$\mathrm{c}$ (vector): Concentration of the vector

\subsubsection{TRANSFORMATION OF COMPETENT E. COLI CELLS}

Transformation of bacteria was used for different purposes: amplification of DNA and plasmids, propagation of correctly ligated DNA fragments, heterologous expression of proteins for purification, and Agrobacteria-mediated plant transformation. Therefore, $1 \mu \mathrm{L}$ (appr. $200 \mathrm{ng}$ ) of plasmid was mixed with $100 \mu \mathrm{L}$ of chemical competent cells $\left(>10^{7}\right.$ colony forming units, Table 3-10, provided by Dr. Ellen Hornung, University of Goettingen, Germany) or, in case of transformation after ligation, the whole ligation volume was mixed with the competent cells. All transformations were incubated on ice for $20 \mathrm{~min}$. Subsequently, the transformation of the cells was stimulated by a heat shock at $42{ }^{\circ} \mathrm{C}$ for $45 \mathrm{~s}$. This treatment loosens the cell wall of the bacteria and enables assimilation of the plasmid of interest. The suspension was chilled on ice for 5 min before $900 \mu \mathrm{L}$ Luria-Bertani (LB) medium (Table 3-8) were added. The cultures were incubated at $37^{\circ} \mathrm{C}$ and $200 \mathrm{rpm}$ for appr. $90 \mathrm{~min}$ to recover and express the antibiotic resistance proteins. In case of ligation, all cells or, in case of plasmid transformation, just a part of the cultures were plated on a LB-agar plate (LB-medium (Table 3-8) with $1.5 \%$ [w/v] microagar (Duchefa Biochemie B.V, Haarlem, Netherlands)) containing the appropriate antibiotics. The plates were incubated overnight at $37^{\circ} \mathrm{C}$. In case of transformation into Agrobacterium tumefaciens (A. tumefaciens), plates and cultures were incubated for $2 \mathrm{~d}$ at $28{ }^{\circ} \mathrm{C}$. 


\subsubsection{COLONY PCR}

Colony PCR was used to identify those cells of a transformation reaction (3.2.3.5) that contain the correct plasmid. Therefore, positively selected colonies were analyzed for the insert of interest on the respective plasmid. A single colony was picked and first streaked on a fresh LB-agar plate (1.5\% [w/v] agarose, (Duchefa Biochemie B.V, Haarlem, Netherlands) in LB-medium (Table 38)) with the respective antibiotics and then dissolved in $20 \mu \mathrm{L}$ PCR reaction solution: $10 \mu \mathrm{L}$ Jump-start Red Taq master mix (Thermo Fisher, Waltham, USA), $1.25 \mathrm{mM}$ primer forward, and $1.25 \mathrm{mM}$ primer reverse in water. The PCR program was set as recommended by the manufacturer. Primers were specific for the insert or specific for the vector backbone flanking the insert. PCR products were analyzed by agarose gel electrophoresis (3.2.3.2) and visualized by UV-light.

\subsubsection{GENERATION OF PLANT MUTANTS}

\subsubsection{GENE EDITING BY CLUSTERED REGULARLY INTERSPACED SHORT PALINDROMIC REPEATS / CRISPR ASSOCIATED PROTEIN 9}

The method of clustered regularly interspaced short palindromic repeats (CRISPR) / CRISPR associated protein 9 (Cas9) is based on a bacterial defense system against viruses. Viral sequence information from a previous attack are stored in the bacterial genome as so-called CRISPR. The molecular tool CRISPR/Cas9 was optimized for scientific usage: the guide RNAs (gRNAs) is designed to contain all information to direct Cas9 to its target sequence for cutting the double stranded protospacer adjacent motif (PAM).

The targets for UGT76E1, UGT76E2, UGT76E11, and UGT76E12 were identified with three different online tools: (i) CRISPRdirect (https://crispr.dbcls.jp/) gives target candidates by comparing the given gene sequence to the selected genome (A. thaliana). The guanine/cytosinecontent of the gRNA should be higher than $40 \%$ and stop-sites for the RNA-Pol III should be avoided. Further, the tool gives the number of off-targets in the genome scored by the position of a mismatch to the PAM. (ii) CRISPR-P (http://crispr.hzau.edu.cn/CRISPR2/) scored candidate targets by the number of off-target sites in the genome (A. thaliana) without judging the position of the mismatch. (iii) The Genetic Pertubation Platform for designing sgRNAs for CRISPRko (http://portals.broadinstitute.org/gpp/public/analysis-tools/sgrna-design) tested the on-target efficiency of candidate targets. Besides misleading results for the human genome (default taxon = human), the "On-Target Efficacy Score" (which is independent of the taxon) gives the power of target sequence for the gene of interest. Values above 0.5 were treated as good candidates, values above 0.7 were judged as very good candidates (oral communication Dr. C. Thurow, Georg-August-University, Goettingen, Germany). 
The best candidates from above (see Table 4-6) were used to design a constructs with four targets and replaced the dummy nucleotides in CRISPR/Cas9 construct according to the protocol from (Xing et al., 2014) (supplemental material S2 and S3). Differing from the protocol, the gRNAs were first ligated into the pJET vector for DNA-amplification and sequencing. The pJETconstructs and the donor vector, pHEE401E (Addgene, Cambridge, USA) were subsequently cut with the endonuclease enzyme BsaI (Thermo Fisher Scientific, Waltham, USA) overnight at $37^{\circ} \mathrm{C}$. The fragments were purified by agarose electrophoresis (3.2.3.2) and extracted from the gel (Nucleospin Gel and PCR Clean-up, Macherey-Nagel, Dueren, Germany). The four fragments were ligated into the acceptor vector corresponding to (Equation 1). The ligations were incubated at room temperature overnight, transformed into ultra-high-competent DH5 $\alpha$ cells (Table 3-10), and selected on kanamycin plates. Positive colonies were re-streaked on both kanamycin and streptomycin (separately), to select for the loss of the streptomycin-resistance gene originally present in the cloning site of pHEE401E. Positive colonies were screened for the presence of a newly introduced HindIII (Target UGT76E11) restriction site, resulting in a fragment of $1580 \mathrm{bp}$. Candidates were confirmed through sequencing with vector-specific sequencing primers (Table 3-12) (Xing et al., 2014) (supplemental material S2 and S3). Correct plasmids were transformed into A. tumefaciens for plant transformation by floral dip (Table 3-10, 3.2.4.3).

\subsubsection{TRANSFORMATION OF AGROBACTERIUM TUMEFACIENS}

Chemically competent A.tumefaciens were provided by Dr. Ellen Hornung (Georg-AugustUniversity, Goettingen, Germany). Competent cells were thawed, mixed with $3 \mu \mathrm{g}$ plasmid DNA, and incubated on ice for $30 \mathrm{~min}$. The transformation of the cells was stimulated by shock freeze at $-80^{\circ} \mathrm{C}$ for $2 \mathrm{~min}$. After the suspension has been thawed again, $900 \mu \mathrm{LB}$ medium were added and the culture was incubated for $3-4 \mathrm{~h}$ at $28{ }^{\circ} \mathrm{C}$ for recovery. Following, all the cells were plated on LB-plates (1.5\% [w/v] agar, (Duchefa Biochemie B.V, Haarlem, Netherlands) in LB-medium (Table 3-8)) supplemented with kanamycin and rifampicin (Table 3-6). The plates were incubated at $28{ }^{\circ} \mathrm{C}$ for $2 \mathrm{~d}$. Resulting clones were used for plant transformation.

\subsubsection{AGROBACTERIUM-MEDIATED TRANSFORMATION OF A. THALIANA}

An important part of the CRISPR/Cas9 gene editing in A.thaliana is the presence of the CRISPR/Cas9-plasmid in the plant. Therefore, A. tumefaciens was used to mediate the gene transfer into the plant embryo by floral dip (Clough \& Bent, 1998). After transformation of the agrobacteria with the plasmid of interest (Table 3-9), several colonies were used to inoculate a pre-culture. $20 \mathrm{ml}$ culture (LB medium (Table 3-8), kanamycin and rifampicin (Table 3-6)) were incubated at $28{ }^{\circ} \mathrm{C}$ and $200 \mathrm{rpm}$ overnight. This pre-culture was used to inoculate $400 \mathrm{ml} \mathrm{LB}$ medium as main-culture. After growing overnight at $28^{\circ} \mathrm{C}$ and $200 \mathrm{rpm}$ the cells were harvested by centrifugation at $8000 \mathrm{x}$ g for $20 \mathrm{~min}$ and the resulting pellet was resolved in $300 \mathrm{ml} 5 \%$ [w/v] 
sucrose solution. Then, $70 \mu \mathrm{l}$ Silwet L-77 (Momentive Performance Materials Inc., Waterford, USA) were added. Subsequently, the flowering inflorescences of Arabidopsis (grown for appr. $4-5$ weeks in long-day conditions, $16 \mathrm{~h}$ light $/ 8 \mathrm{~h}$ dark, $22^{\circ} \mathrm{C}$ ) were dipped into this bacteria suspension. The plants were covered overnight. The flower dipping transformation was repeated one week later. Positive T1 transformed lines were selected by hygromycin (Table 3-6).

\subsubsection{QUANTITATIVE REAL-TIME PCR EXPRESSION ANALYSIS}

To analyze the expression status of selected genes quantitative real-time PCR (qPCR) was used. It determines the amount of gene product in a biological sample. The amounts were determined in a cDNA pool, which has been synthesized from total RNA of A. thaliana (3.2.2.2). The analysis monitors the amount of DNA synthesized in a PCR reaction in real-time. Here, the reactions were carried out in an iQ5 real-time PCR detection systems (Bio-Rad Laboratories GmbH, Muenich, Germany) with the appropriate filter for the emitting wavelength of the fluorescent chromophore SYBR-green (Thermo Fisher Scientific, Waltham, USA). The molecule intercalates into the DNA formed during the reaction and gives fluorescent signals, which are detected during the PCR run at the end of every cycles. The amount of template in the cDNA pool defines how many cycles will be needed for that particular PCR-product to reach the exponential phase. Likewise, higher and earlier fluorescent signals are detected in the PCR when the gene had been expressed with higher rates. The amount of transcript of different genes under certain conditions can be quantified and compared by the number of cycles which it took to synthesis a certain amount of DNA in the qPCR. The obtained qPCR data of genes of interest were compared to the expression of the reference gene actin 8 (Atlg49240). This gene has been chosen because its expression is not altered in the response to wounding and not directly involved in genetic JA-response.

Here, the Takyon No ROX SYBR Mastermix (Kaneka Eurogentec S.A., Seraing, Belgium) was used as a $2 \mathrm{x}$ ready to use mix. For a reaction $10 \mu \mathrm{L}$ Takyon No ROX SYBR Mastermix, $2 \mu \mathrm{L}$ primer mix $(25 \mathrm{mM}), 5.5 \mathrm{~mL}$ water, and $2.5 \mu \mathrm{L}$ cDNA $(300-500 \mathrm{ng} / \mu \mathrm{L})$ were mixed in PCR stripes with flat caps. The reaction was performed with 40 cycles. Special qPCR-primers were designed with the online tool primer3plus (https://primer3plus.com/cgi-bin/dev/primer3plus.cgi). The DNA sequences of UGT76E1, UGT76E2, UGT76E11, UGT76E12, and UGT74F1 were submitted to primer3prefold (https://primer3plus.com/cgi-bin/dev/primer3prefold.cgi) and regions tending to form secondary structure were identified and neglected for primer design. These results were send to primer3plus to score candidate primer pairs (server setting qPCR). The best primer pairs for every gene were tested by PCR. Positive testing means that one specific DNA product is formed exclusively and no unspecific DNA fragments are co-amplified. Positive and specific primers (Table 3-12) were used for qPCR. 


\subsubsection{PURIFICATION OF HETERLOGOUS EXPRESSED PROTEINS}

\subsubsection{CELL DISRUPTION OF E. COLI CELLS}

After heterologous protein expression (see 3.2.2.4), the bacterial cells were harvested and disrupted for the following protein purification. Therefore, a cell pellet was resuspended in the His A buffer (Table 3-16 - Table 3-20). Appr. $3 \mathrm{~mL}$ buffer per $1 \mathrm{~g}$ cells were used to get a dense suspension for effective disruption. This suspension was treated with $1 \mathrm{mg} / \mathrm{mL}$ lysozyme from chicken egg white (Merck KGaA, Darmstadt, Germany) and $0.01 \mathrm{mg} / \mathrm{mL}$ DNAse I from bovine pancreas (Merck KGaA, Darmstadt, Germany) and stirred for $20 \mathrm{~min}$. Following that pretreatment, small volumes $(<30 \mathrm{~mL})$ were disrupted by pulsed ultrasonic treatment (Branson Sonifier Cell Disruptor B15, Branson Ultrasonics Corporation, Danbury, USA) at $40 \%$ power and $50 \%$ impulse level for $5 \times 1$ min and cooled down in between for $1 \mathrm{~min}$. Larger volumes of around $120 \mathrm{~mL}$ were disrupted by high pressure in a Microfluidizer device (Fluidizer 110L, Microfluidic, Westwood, USA). The crude lysate was centrifuged at $50000 \mathrm{xg}$ for at least $30 \mathrm{~min}$ to pellet the cell debris. The resulting cleared lysate was introduced to protein purification.

\subsubsection{RECOMBINANT PROTEIN PURIFICATION}

For preparing protein purification, the protein parameters were calculated with the ProtParam online tool (https://web.expasy.org/protparam/). Calculated properties were: the molecular weights (MWs), the isoelectric points (pIs), and the specific extinction coefficients at $280 \mathrm{~nm}$. Here, the calculations take into account the numbers of the aromatic amino acids tryptophan, tyrosine and cysteine and the MW. Furthermore, the amino acid sequences were analyzed for signal peptides by SignalP (http://www.cbs.dtu.dk/services/SignalP/), for target peptides by TargetP (http://www.cbs.dtu.dk/services/TargetP/), and for transmembrane domains with the TMHMM tool (http://www.cbs.dtu.dk/services/TMHMM/).

Protein purification was applied for proteins tagged with an N-terminal hexa-histidine tag (HisTag), which were heterlogously expressed in Ecoli. A two-step purification strategy of immobilized metal affinity chromatography (IMAC) and size exclusion chromatography (SEC) was used. The ÄKTAprime plus systems (GE Healthcare (Little Chalfont, UK) was used for protein purification at $4{ }^{\circ} \mathrm{C}$. The IMAC was done with $5 \mathrm{~mL}$ HisTrap columns (GE Healthcare (Little Chalfont, UK) or 5 mL HisTALON columns (Takara Bio Europe, Saint-Germain-en-Laye, France). The SEC was performed with a S75 Sepharose gel filtration column (GE Healthcare, Little Chalfont, UK).

The first purification step was an IMAC. Here, the purification bases on the affinity of the imidazole groups of the histidine amino acids of the HisTag of the protein to form octahedral complexes with incomplete-chelated bivalent cations $\left(\mathrm{Ni}^{2+}\right.$ or $\left.\mathrm{Co}^{2+}\right)$ on the resin. Meaning that 
the tagged proteins were bound to the equilibrated column while all the other proteins of the cleared lysate flowed through the column. This protein loading was done with a flowrate of $1.5 \mathrm{~mL} / \mathrm{min}$. Loading conditions were also used to wash all unspecific proteins from the matrix. In some cases, this washing was not sufficient to remove unspecific bound proteins. Especially, bacterial chaperons are often bound to the proteins of interests directly and rigidly. Those chaperones are native bacterial proteins assisting in correct protein folding during protein biosynthesis and require metabolic energy to be released again. In that cases, a second washing step was applied: a wash buffer (50 mM Tris pH 7.5, $100 \mathrm{mM} \mathrm{NaCl,} 2 \mathrm{mM}$ dithiothreitole (DTT), $5 \mathrm{mM}$ ATP, $5 \mathrm{mM} \mathrm{MgCl}_{2}, 20 \mathrm{mM}$ Imidazole, Table 3-21) was used to elute strongly interacting proteins from the matrix or the tagged protein. For protein elution, the His-tagged proteins was displaced from the complex by imidazole (150 mM for HisTrap and $40 \mathrm{mM}$ for HisTALON). At the end of every purification run, a washing with imidazole was performed (500 mM for HisTrap and $200 \mathrm{mM}$ for HisTALON) to elute every protein from the column and to start recovery of the column. The entire purification process was recorded by UV-Vis spectroscopy at $280 \mathrm{~nm}$. Samples of all purification steps were analyzed by denaturating sodium dodecyl sulfate (SDS)polyacrylamide gel electrophoresis (PAGE).

For a second purification step a SEC was used. SEC separates proteins by their specific radius of gyrations, which is the mean of all the distances from the surface of the protein to the center of gravity. Generally, radius of gyration corresponds to the size of the protein. It ensure that copurified proteins from the IMAC purification are separated in a second purification step by a different chromatographic principle (SEC). Technically, the sepharose matrix of the gel filtration has a defined pore size in which molecules might or might not diffuse corresponding to their radius of gyration - their size. Consequently, smaller molecules diffuse in pores and have more interaction. Physically, longer distance at a constant speed equals more time. Hence, bulky molecules elute earlier from the gel filtration than smaller ones. Like this, the elution volume of a specific protein from the gel filtration column can be calculated by calibrating the column (Equation 2). Here, the real protein sizes of the expressed amino-acid chain with HisTag were used for calculations. The HiLoad 16/600 Superdex 75 prep grade (GE Healthcare, Little Chalfont, UK) is capable to separate proteins up to $70 \mathrm{kDa}$. Again, protein elution was monitored

Equation 2

$$
\text { Volume }_{S 75}(\text { protein })=\frac{\log _{10}(\mathrm{MW}(\text { protein }))-6.9219}{(-0.0352)}
$$

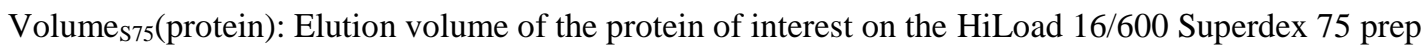
grade (GE Healthcare, Little Chalfont, UK)

MW(protein): Molecular weight of the protein of interest 
by UV-Vis spectroscopy at $280 \mathrm{~nm}$ and SDS-PAGE. For further use and tests, the homogeneous proteins were concentrated to $>1 \mathrm{mg} / \mathrm{mL}$ with concentrators (Spin-X UF6, $30 \mathrm{kDa}$ cut-off, Corning B.V. Life Science, Amsterdam, Netherlands), flash-frozen in liquid $\mathrm{N}_{2}$ and stored at $80{ }^{\circ} \mathrm{C}$.

\section{Table 3-16: Buffer systems for protein purification of UGT76E1}

Specific buffer systems were used to purify heterologous expressed proteins. His A buffer was used for equilibrating the HisTALON Superflow Cartridge (Takara Bio Europe, Saint-Germain-en-Laye, France), loading the cleared lysate, and washing. His B was the elution buffer to displace proteins from the affinity columns. Following, proteins were purified with size exclusion chromatography. The gel filtration buffer was a low-salt buffer without special additives.

\begin{tabular}{|c|c|c|}
\hline \multicolumn{3}{|c|}{ His A } \\
\hline & $\mathrm{mM}$ & Tris/HCl pH 9.0 \\
\hline & $\mathrm{mM}$ & $\mathrm{NaCl}$ \\
\hline 0.1 & $\%$ & Tween 20 \\
\hline \multicolumn{3}{|c|}{ His B } \\
\hline & $\mathrm{mM}$ & Tris/HCl pH 9.0 \\
\hline 100 & $\mathrm{mM}$ & $\mathrm{NaCl}$ \\
\hline 0.1 & $\%$ & Tween 20 \\
\hline 200 & $\mathrm{mM}$ & Imidazole \\
\hline \multicolumn{3}{|c|}{ Gel filtration } \\
\hline & $\mathrm{mM}$ & Tris/HCl pH 9.0 \\
\hline 100 & $\mathrm{mM}$ & $\mathrm{NaCl}$ \\
\hline
\end{tabular}

Table 3-17: Buffer systems for protein purification of UGT76E2

Specific buffer systems were used to purify heterologous expressed proteins. His A was used for equilibrating the HisTrap HP (GE Healthcare, Little Chalfont, UK), loading the cleared lysate, and washing. His B was the elution buffer to displace proteins from the affinity columns. Following, proteins were purified with size exclusion chromatography. The gel filtration buffer was a low-salt buffer without special additives.

\begin{tabular}{rll}
\hline \multicolumn{2}{c}{ UGT76E2 } & His A \\
\hline 50 & $\mathrm{mM}$ & Tris/HCl pH 8.0 \\
100 & $\mathrm{mM}$ & NaCl \\
0.1 & $\%$ & Tween 20 \\
\hline \multicolumn{2}{c}{ UGT76E2 } & His B \\
\hline 50 & $\mathrm{mM}$ & Tris/HCl pH 8.0 \\
100 & $\mathrm{mM}$ & NaCl \\
0.1 & $\%$ & Tween 20 \\
500 & $\mathrm{mM}$ & Imidazole \\
\hline UGT76E2 & Gel filtration \\
\hline 50 & $\mathrm{mM}$ & Tris/HCl pH 8.0 \\
100 & $\mathrm{mM}$ & NaCl \\
\hline
\end{tabular}


Table 3-18: Buffer systems for protein purification of UGT76E11

Specific buffer systems were used to purify heterologous expressed proteins. His A was used for equilibrating the affinity columns, loading the cleared lysate, and washing. His B was the elution buffer to displace proteins from the affinity columns. Following, proteins were purified with size exclusion chromatography. The gel filtration buffer was a low-salt buffer without special additives.

\begin{tabular}{|c|c|c|}
\hline \multicolumn{3}{|c|}{ His A } \\
\hline & $\mathrm{mM}$ & Tris/HCl pH 7.5 \\
\hline & $\mathrm{mM}$ & $\mathrm{NaCl}$ \\
\hline & $\%$ & Glycerol \\
\hline 0.1 & $\%$ & Tween 20 \\
\hline \multicolumn{3}{|c|}{ His B } \\
\hline & $\mathrm{mM}$ & Tris/HCl pH 7.5 \\
\hline & $\mathrm{mM}$ & $\mathrm{NaCl}$ \\
\hline 10 & $\%$ & Glycerol \\
\hline 0.1 & $\%$ & Tween 20 \\
\hline 500 & $\mathrm{mM}$ & Imidazole \\
\hline \multicolumn{3}{|c|}{ Gel filtration } \\
\hline & $\mathrm{mM}$ & Tris/HCl pH 7.5 \\
\hline
\end{tabular}

Table 3-19: Buffer systems for protein purification of UGT76E12

Specific buffer systems were used to purify heterologous expressed proteins. His A was used for equilibrating the affinity columns, loading the cleared lysate, and washing. His B was the elution buffer to displace proteins from the affinity columns. Following, proteins were purified with size exclusion chromatography. The gel filtration buffer was a low-salt buffer without special additives.

\begin{tabular}{|c|c|c|}
\hline \multicolumn{3}{|c|}{ His A } \\
\hline & $\mathrm{mM}$ & Tris/HCl pH 8.0 \\
\hline & $\mathrm{mM}$ & $\mathrm{NaCl}$ \\
\hline & $\%$ & Glycerol \\
\hline \multicolumn{3}{|c|}{ His B } \\
\hline & $\mathrm{mM}$ & Tris/HCl pH 8.0 \\
\hline 100 & $\mathrm{mM}$ & $\mathrm{NaCl}$ \\
\hline 10 & $\%$ & Glycerol \\
\hline 500 & $\mathrm{mM}$ & Imidazole \\
\hline \multicolumn{3}{|c|}{ Gel filtration } \\
\hline 50 & $\mathrm{mM}$ & Tris/HCl pH 8.0 \\
\hline 100 & $\mathrm{mM}$ & $\mathrm{NaCl}$ \\
\hline
\end{tabular}

Table 3-20: Buffer systems for protein purification of UGT74F1

Specific buffer systems were used to purify heterologous expressed proteins. His A was used for equilibrating the affinity columns, loading the cleared lysate, and washing. His B was the elution buffer to displace proteins from the affinity columns. Following, proteins were purified with size exclusion chromatography. The gel filtration buffer was a low-salt buffer without special additives.

\begin{tabular}{|c|c|c|}
\hline \multicolumn{3}{|c|}{ His A } \\
\hline & $\mathrm{mM}$ & Tris/HCl pH 8.0 \\
\hline & $\mathrm{mM}$ & $\mathrm{NaCl}$ \\
\hline & $\%$ & Tween 20 \\
\hline \multicolumn{3}{|c|}{ His B } \\
\hline 50 & $\mathrm{mM}$ & Tris/HCl pH 8.0 \\
\hline 100 & $\mathrm{mM}$ & $\mathrm{NaCl}$ \\
\hline 0.1 & $\%$ & Tween 20 \\
\hline 500 & $\mathrm{mM}$ & Imidazole \\
\hline \multicolumn{3}{|c|}{ Gel filtration } \\
\hline 50 & $\mathrm{mM}$ & Tris/HCl pH 8.0 \\
\hline 100 & $\mathrm{mM}$ & $\mathrm{NaCl}$ \\
\hline
\end{tabular}




\section{Table 3-21: Wash buffer for protein purification}

To remove unspecific proteins from the affinity purification column, a special wash buffer was used. It was applied between loading of proteins and specific elution of the proteins of interests. Dithiothreitol, DTT; adenine triphosphate, ATP.

\begin{tabular}{rll}
\hline His wash buffer & \\
\hline 50 & $\mathrm{mM}$ & Tris/HCl pH 7.5 \\
100 & $\mathrm{mM}$ & $\mathrm{NaCl}$ \\
2 & $\mathrm{mM}$ & $\mathrm{DTT}$ \\
5 & $\mathrm{mM}$ & $\mathrm{ATP}$ \\
5 & $\mathrm{mM}$ & $\mathrm{MgCl}_{2}$ \\
20 & $\mathrm{mM}$ & Imidazole \\
\hline
\end{tabular}

\subsubsection{PROTEIN SEPARATION BY SDS-PAGE}

SDS-PAGE (Davis, 1964; Kellenberger, 1968; Laemmli, 1970; Ornstein, 1964) was used to record the protein purification process. The samples were sonified and boiled before the proteins were separated by SDS-PAGE. For SDS-PAGE, samples of $20 \mu \mathrm{L}$ were mixed with $20 \mu \mathrm{L}$ of $2 \mathrm{x}$ Laemmli sample buffer (Table 3-22). Thereof, 5-10 $\mu \mathrm{L}$ were loaded on a $10 \%$ acryl amide gel (Table 3-23). For molecular weight determination $5 \mu \mathrm{L}$ Unstained Molecular Weight Marker (MBI Fermentas, St. Leon Roth, Germany) were loaded on a separate lane of the gel. The separation itself was performed in a Mini-Protean3 electrophoresis system (Bio-Rad Laboratories $\mathrm{GmbH}$, Munich, Germany) at $30 \mathrm{~mA}$ per gel until the running front was just run out of the gel. The proteins in the gel were visualized with Coomassie Brillant Blue staining solution, which is prepared of $1 \mathrm{~mL}$ of Coomassie staining stock solution in appr. $50 \mathrm{~mL}$ of fixation solution (Table 3-25). The gel was boiled in the staining solution and incubating for $10 \mathrm{~min}$ for protein staining. For de-staining the gel was several times boiled in water and incubated with a dye-absorbing tissue for $10 \mathrm{~min}$.

For SDS-PAGE of whole bacterial cells, aliquots of the expression culture (Equation 3) were centrifuged at $10000 \mathrm{xg}$ for $4 \mathrm{~min}$. The pellet was dissolved in $50 \mu \mathrm{L}$ water and $50 \mu \mathrm{L} 2 \mathrm{x}$ Laemmli sample buffer. The samples were sonified and boiled before the proteins were separated by SDS-PAGE as before.

$$
\frac{0.5 \mathrm{~mL}}{\mathrm{OD}_{600}}=\text { Volume (aliquot) }
$$

$\mathrm{OD}_{600}$ : Optical density of the bacterial culture at $600 \mathrm{~nm}$ Volume (aliquot): Volume of the aliquot in $\mathrm{mL}$ 
Table 3-22: Composition of 2x Laemmli sample buffer

Dithiothreitole (DTT), sodium dodecyl sulfate (SDS). Volume per volume, v/v; weight per volume, w/v.

$\begin{aligned} 100 \mathrm{mM} & \text { Tris/HCl pH } 6.8 \\ 200 \mathrm{mM} & \text { DTT } \\ 4 \%[\mathrm{w} / \mathrm{v}] & \text { SDS } \\ .002 \%[\mathrm{w} / \mathrm{v}] & \text { Bromphenole blue } \\ 40 \%[\mathrm{v} / \mathrm{v}] & \text { Glycerol }\end{aligned}$

Table 3-23: Composition of $10 \%$ SDS-PAGE gel

Tetramethylethylenediamine (TEMED). The volume of the recipe is sufficient for $4 \mathrm{x} 0.75 \mathrm{~mm}$ gels (BioRad Laboratories GmbH, Munich, Germany).

\begin{tabular}{lcc}
\hline & $4 \%$ Stacking gel $(\mathrm{mL})$ & $10 \%$ Separation gel $(\mathrm{mL})$ \\
\hline Separation gel buffer & - & 4.25 \\
Stacking gel buffer & 1.90 & - \\
Water & 4.50 & 6.92 \\
(Bis) acrylamide $(30 \%[\mathrm{w} / \mathrm{v}])$ & 1.00 & 5.67 \\
Ammonium persulfate & $70 \cdot 10^{-3}$ & $153 \cdot 10^{-3}$ \\
TEMED & $7.5 \cdot 10^{-3}$ & $17 \cdot 10^{-3}$ \\
\hline
\end{tabular}

Table 3-24: Composition of stock buffers for SDS-PAGE

\begin{tabular}{cl}
\hline Stacking gel buffer & \\
\hline $0.5 \mathrm{M}$ & Tris/HCl pH 6.8 \\
$0.1 \%[\mathrm{w} / \mathrm{v}]$ & SDS \\
\hline Separation gel buffer \\
\hline $1.5 \mathrm{M}$ & Tris/HCl $\mathrm{pH} 8.8$ \\
$0.1 \%[\mathrm{w} / \mathrm{v}]$ & SDS \\
\hline
\end{tabular}

Table 3-25: Coomassie staining solution

Volume per volume, v/v; weight per volume, w/v.

\begin{aligned} & \hline \multicolumn{2}{c}{ Coomassie staining stock solution } \\ & \hline $0.25 \%[\mathrm{w} / \mathrm{v}]$ Brilliant blue G-250 \\ & $0.25 \%[\mathrm{w} / \mathrm{v}]$ Brilliant blue R-250 \\ & In $100 \%[\mathrm{v} / \mathrm{v}]$ Ethanol \\ & \hline Fixation solution \\ & \hline $5 \%[\mathrm{v} / \mathrm{v}]$ Acetic acid \\ & $10 \%[\mathrm{v} / \mathrm{v}]$ Ethanol \\ & $85 \%[\mathrm{v} / \mathrm{v}] \mathrm{H}_{2} \mathrm{O} \\ &$\hline\end{aligned}

\subsubsection{PROTEIN DETECTION BY WESTERN BLOT}

His-tagged proteins were detected in whole bacterial cell after protein expression. After SDSPAGE of the samples, the stacking gel was removed and the proteins in separation gel were transferred onto a nitrocellulose membrane (Roti-NC, pore size $2 \mu \mathrm{m}$, Carl Roth \& Co., Karlsruhe, Germany). Therefore, a Mini-Protean3 electrophoresis system was used with a Mini Trans-Blot Cell (both Bio-Rad Laboratories GmbH, Munich, Germany). The transfer was carried out in wet conditions in transfer buffer (25 mM Tris, $192 \mathrm{mM}$ glycine, $20 \%$ methanol [v/v]) at $240 \mathrm{~mA}$ and $80 \mathrm{~V}$ for $2 \mathrm{~h}$. The transfer cassette was dismantled and membrane was checked for successful 
protein blotting by staining with Ponceau $\mathrm{S}(0.1 \%[\mathrm{w} / \mathrm{v}]$ in water : acetic acid, [99: $1, \mathrm{v} / \mathrm{v}]$ for 5 min. The staining was removed with water before the membrane was incubated in $0.05 \%$ [v/v] Tween 20, $10 \mathrm{mM}$ Tris/HCl, $\mathrm{pH} 7.9,150 \mathrm{mM} \mathrm{NaCl}$ (Tween-Tris buffered saline, T-TBS) containing $3 \%$ milk powder (blotting grade, Carl Roth \& Co., Karlsruhe, Germany). The blocking was performed at room temperature for $1 \mathrm{~h}$. Following, the membrane was incubated within a solution of the primary antibody $(0.1 \mu \mathrm{g} / \mathrm{mL}$ in T-TBS, $3 \%$ milk powder, Tetra-His Antibody, Quiagen, Hilden, Germany) at $10 \mathrm{rpm}$ and $4{ }^{\circ} \mathrm{C}$ overnight. Next, the primary antibody solution was removed and membrane was washed four times with T-TBS, $3 \%$ milk powder at room temperature on a rotary shaker for $10 \mathrm{~min}$. Primary antibody-antigen conjugates were detected using an alkaline phosphatase-conjugated secondary antibody (goat anti mouse lgG, Merck KGaA, Darmstadt, Germany) diluted 1:30000 in T-TBS, 3\% milk powder. The membrane was incubated with this secondary antibody solution at room temperature on a rotary shaker for $1 \mathrm{~h}$. The antibody solution was removed and membrane were washed with T-TBS for $10 \mathrm{~min}$ (two times) and for $5 \mathrm{~min}$ (two times) with Tris buffered saline (TBS). For activity staining mediated by the alkaline phosphatase (AP), the membrane was equilibrated in AP buffer (100 mM Tris/ $\mathrm{HCl} \mathrm{pH} 9.5,100 \mathrm{mM} \mathrm{NaCl}, 5 \mathrm{mM} \mathrm{MgCl} 2$ ) and incubated with staining solution (15 mL AP buffer, $49.5 \mu \mathrm{L}$ Nitro-blue-tetrazolium-chloride ( $50 \mathrm{mg} / \mathrm{mL}$ in $70 \%$ dimethylformamide), $99 \mu \mathrm{L}$ 5-bromo-4-chloro-3'-indolyphosphate p-toluidine salt $(50 \mathrm{mg} / \mathrm{mL}$ in dimethylformamide) in the dark for 30-300 min. The staining reaction was stopped by transferring into water.

\subsubsection{ENZYME CHARACTERIZATION}

\subsubsection{ProteIn ACTIVITY ASSAY}

A fast and simple protein activity assay was performed to monitor the activity of an enzyme during protein purification, to check enzymatic activity towards new potential substrates, and to test the activity of so far not characterized UGT-enzymes. Therefore, reactions were set up in $100 \mu \mathrm{L}$ assay volume containing $10 \mu \mathrm{g}$ enzyme, $0.1 \mathrm{mM}$ substrate and $0.5 \mathrm{mM}$ UDP-Glc. The assay were incubated at room temperature for $1 \mathrm{~h}$ and stopped by adding $100 \mu \mathrm{L}$ ACN. The solvent precipitates. The samples were centrifuged at $20000 \mathrm{x} g$ at room temperature for $5 \mathrm{~min}$ and $50 \mu \mathrm{L}$ of the supernatant were transferred into glass vials. The substrates and products of the reaction were analyzed by LC coupled to mass spectrometer (LC-MS), 3.2.8.4).

\subsubsection{DETERMINATION OF OPTIMAL TEMPERATURE AND PH VALUES}

The enzymatic characterization of UGT76E1, UGT76E2, UGT76E11, and UGT76E12 required the determination of optimal parameters for the reaction, like $\mathrm{pH}$ and temperature optima.

The enzymatic product formation was evaluated at different $\mathrm{pH}$ conditions. The reactions were performed in $200 \mu \mathrm{L}$ solutions containing $0.1 \mathrm{mM} \omega$-hydroxy-hexadecanoic acid ( $\omega$-OH-16:0, 
dissolved in ACN), $0.5 \mathrm{mM}$ UDP-Glc, and $30 \mu \mathrm{g}$ pure enzyme. To test a broad $\mathrm{pH}$ range from 2 to 12 with the high buffering capacity, the Britton-Robinson buffer (BRB) system (Britton \& Robinson, 1931) was used. Tested were $13 \mathrm{pH}$ conditions: $\mathrm{pH}$ 3.0, 4.0, 5.0, 6.0, 7.0, 7.5, 8.0, 8.5, 9.0, 9.5, 10.0, 11.0. All reactions were started by adding the enzyme and incubated at $25{ }^{\circ} \mathrm{C}$ for $1 \mathrm{~h}$. The reactions were stopped by adding $50 \mu \mathrm{L}$ ACN. After centrifugation at $20000 \mathrm{x} g$ at room temperature for $5 \mathrm{~min}, 50 \mu \mathrm{L}$ of the supernatant were used for detecting relative amounts of $\omega-O$ glucosyl-hexadecanoic acid ( $\omega-O$-Glc-16:0) by LC-MS.

The optimal reaction temperature of the UGTs was investigated similarly just at fixed $\mathrm{pH}$ and different temperatures: $4{ }^{\circ} \mathrm{C}, 10{ }^{\circ} \mathrm{C}, 15^{\circ} \mathrm{C}, 20{ }^{\circ} \mathrm{C}, 25{ }^{\circ} \mathrm{C}, 30{ }^{\circ} \mathrm{C}, 35{ }^{\circ} \mathrm{C}, 42{ }^{\circ} \mathrm{C}, 50{ }^{\circ} \mathrm{C} .0 .1 \mathrm{mM} \omega$ OH-16:0, $5 \mathrm{mM}$ UDP-Glc were mixed in $50 \mathrm{mM}$ Tris- $\mathrm{HCl}, 100 \mathrm{mM} \mathrm{NaCl}$ buffer at $\mathrm{pH} 8.5$ in a volume of $200 \mu \mathrm{L}$. These reactions were pre-incubated at the indicated reactions temperature for $10 \mathrm{~min}$. Reactions at temperature lower than room temperature were incubated in water baths and reactions at temperatures above room temperature were incubated in heating blocks. $30 \mu \mathrm{g}$ enzyme was added and the reactions were carried out for $1 \mathrm{~h}$ before the reactions were terminated by the addition of $50 \mu \mathrm{L} \mathrm{ACN}$. The reaction products were analyzed as before.

\subsubsection{ANALYSIS OF THE SUBSTRATE SPECIFICITY OF UGTS BY USING UDP- ${ }^{14} \mathrm{C}(\mathrm{U})-\mathrm{GLC}$}

The substrate specificity of the UGTs was tested against different substrates with free hydroxy and/or carboxy groups. To describe the product formation in a half quantitative way radioactive product determination was used to contrast a set of different aglycones for their performance as substrate of one enzyme. Hereby, the Glc moiety is uniformly ${ }^{14} \mathrm{C}$ labelled $\left({ }^{14} \mathrm{C}(\mathrm{U})\right.$-Glc) one, which is transferred onto the aglycone upon catalysis. Like this, every turnover produces the same signal intensity and the signal intensities of all products can be compared.

Different aglycones (Table 3-26) were tested as substrates for UGT76E1, UGT76E2, UGT76E11, UGT76E12, and UGT74F1. The enzymatic reactions were carried out in a total volume of $20 \mu \mathrm{L}$ with $60 \mu \mathrm{M}$ substrate, $3.3 \mu \mathrm{M}$ labeled UDP- ${ }^{14} \mathrm{C}(\mathrm{U})-\mathrm{Glc}(0.02 \mu \mathrm{Ci}), 28 \mu \mathrm{M}$ UDP-Glc, and $5 \mu \mathrm{g}$ enzyme. The reaction assays and one negative control with inactivated enzyme were started by adding the enzyme and incubating at $25^{\circ} \mathrm{C}$ for $1 \mathrm{~h} .30 \mu \mathrm{L}$ of n-butanol were added to stop the reactions and to extract the metabolites (modified from Huang et al., 2015; Suzuki et al., 2007). This solution was mixed thoroughly and centrifuged at $13200 \mathrm{x}$ g for $5 \mathrm{~min}$. The organic phase was taken and transferred into a new reaction tube. The residue was extracted again, mixed, and centrifuged as before. Both extractions were pooled and evaporated under stream of nitrogen. The residue was resolved in $20 \mu \mathrm{L} 70 \%$ [v/v] ethanol and spotted on thin-layer chromatography (TLC) plates (Silica gel 60 plate, Merck KGaA, Darmstadt, Germany). The TLC-plates were placed in an equilibrated TLC-chamber and run in dichlormethane : methanol : water $[80: 20: 2$, 
v/v/v] (modified from Augustin et al., 2012). Then, the plates were air-dried and covered with cling film and placed on phosphor screens in storage cassettes (Fujifilm Corporation Tokyo, Japan). After $3 \mathrm{~d}$ of incubation, the screens were scanned with a FLA-3000 fluorescent image analyzer (Fujifilm Corporation Tokyo, Japan). Data analysis was done with the "Bildanalyseprogramm1.0.4.6" (Datinf GmbH, Tuebingen, Germany) at a picture contrast of 4220 - 41200 pixel. Signal intensities were determined as within a signal detection boxes. Background signals were subtracted by a neighboring box. Product signals were calculated as product signal minus background over area.

Table 3-26: Substances, which were tested as substrates for the UGTs.

\begin{tabular}{|c|c|c|c|}
\hline \multicolumn{4}{|c|}{ Substrates } \\
\hline 1 & Hexadecanoic acid (16:0) & 12 & Gibberellic acid (GA) \\
\hline 2 & $\omega$-Hydroxy-hexadecanoic acid ( $\omega-\mathrm{OH}-16: 0)$ & 13 & cis-(+)-12-Oxo-phytodienoic acid (12-OPDA) \\
\hline 3 & 2-Hydroxy-hexadecanoic acid (2-OH-16:0) & 14 & Jasmonic acid (JA) \\
\hline 4 & 3-Hydroxy-hexadecanoic acid (3-OH-16:0) & 15 & 12-Hydroxy-JA (12-OH-JA) \\
\hline 5 & Hexadecanol $(\mathrm{OH}-\mathrm{C} 16)$ & 16 & 11-Hydroxy-JA (11-OH-JA) \\
\hline 6 & Benzoic acid (BA) & 17 & 12-Hydroxy-JA-methyl ester (12-OH-JA-ME) \\
\hline 7 & Salicylic acid (SA) & 18 & Quercetin \\
\hline 8 & Pipecolic acid (Pip) & 19 & Dihydro-myricetin(dh-myricetin) \\
\hline 9 & Indole-carboxylic acid (ICA) & 20 & Dihydro-kaempferol(dh-kaempferol) \\
\hline 10 & Abscisic acid (ABA) & 21 & Control with inactivted enzyme \\
\hline 11 & Zeatin & & \\
\hline
\end{tabular}

\subsubsection{DETERMINATION OF THE KINETIC PARAMETERS}

To record steady-state kinetics, a coupled spectrophotometric assay has been modified from (Brown et al., 2012). UGTs catalyze the transfer of a Glc-moiety from the donor UDP-Glc to the hydroxyl-aglycone releasing the respective glycone and UDP. Via the co-product UDP, the UGTreaction is coupled to the pyruvate kinase (PK) and lactate dehydrogenase (LDH) to record the UGT reaction kinetics in $1: 1: 1$ stoichiometry: The PK phosphorylates UDP to UTP while metabolizing phosphoenolpyruvate (PEP) to pyruvate. $\mathrm{LDH}$ catalyzes the reduction of pyruvate to lactate by oxidizing nicotinamide adenine dinucleotide (NADH) to $\mathrm{NAD}^{+}$, which shows in contrast to NADH no absorption maximum at $340 \mathrm{~nm}$. All measurements were performed in biological triplicates in Quartz SUPERSIL cuvettes (Hellma Analytics, Muellheim, Germany) in a V-630 UV/Vis Spectrophotometer (JASCO Corporation, Hachioji, Japan). The detailed reaction mixture is given in Table 3-27. UGT76E1 had be stabilized with bovine serum albumin (BSA, $0.13 \%[\mathrm{w} / \mathrm{v}])$. For the assay, the NADH stock-solution was prepared freshly in the gel filtration buffer of the respective enzyme (Table 3-16 - Table 3-20). The exact concentration of NADH was determined in 1/100 dilutions in the photometer with the Lambert Beer law (Equation 4). Therewith, a 2x master mix was prepared to reduce pipetting errors between the different measurements. The setup was blanked with an empty cuvette. The master mix was added to the 
substrate and the buffer in the cuvette. This solution was checked to have an absorption at $340 \mathrm{~nm}$ of appr. 0.9 - the calculated value for the working concentration of $0.15 \mathrm{mM} \mathrm{NADH}$ in the cuvette is 0.96 (Equation 4). To start the reactions, the enzyme was added and mixed carefully by pipetting. The reaction was recorded at $340 \mathrm{~nm}$ for $300-600 \mathrm{~s}$ depending on the velocity of the reaction. The linear slope of the reaction was determined with the Spectra Manager II Software (JASCO Corporation, Hachioji, Japan). A high concentration of UDP was used to was ensured that the rate limiting reaction is the UGT reaction. For evaluation, the reaction velocities were plotted against the given substrate concentration by the SIMGA-Plot software. Finally, the velocities were calculated to $\mathrm{k}_{\mathrm{cat}}$ values by a modified Lambert-Beer equation (Equation 5)

$c($ analyte): Concentration of the analyte of interest

$$
c(\text { analyte })=\frac{\text { Absorption }}{\varepsilon(\text { analyte }) \cdot 1}
$$

Equation 4

$\varepsilon$ (analyte): Specific extinction coefficient of the analyte

1: Path length, for cuvettes normally $=1 \mathrm{~cm}$

$$
\mathrm{k}_{\mathrm{cat}}=\frac{\mathrm{V}_{\max }}{[\mathrm{E}]}=\frac{\Delta \text { Absorption }}{\varepsilon(\mathrm{NADH}) \cdot \mathrm{l} \cdot[\mathrm{E}] \cdot \mathrm{t}}=\left[\mathrm{s}^{-1}\right]
$$

Equation 5

$\mathrm{k}_{\mathrm{cat}}$ : Maximal turnover number

$\mathrm{V}_{\max }$ : Maximal reaction velocity; change of absorption per min

[E]: Concentration of enzyme

$\varepsilon(\mathrm{NADH})$ : Specific extinction coefficient of NADH, $6220 \mathrm{M}^{-1} \mathrm{~cm}^{-1}$

1: Path length (for the used cuvettes) $=1 \mathrm{~cm}$

$\mathrm{t}$ : Reaction time

Table 3-27: Reaction mixture of the coupled spectrophotometric assay.

Phosphoenolpyruvate, PEP; pyruvate kinase, PK; lactate dehydrogenase, LDH; uridine diphosphate glucose, UDP-Glc; nicotinamide adenine dinucleotide, NADH; UDP-dependent glycosyltransferase,

\begin{tabular}{|c|c|c|c|c|c|}
\hline \multicolumn{2}{|c|}{ Volume } & \multicolumn{2}{|l|}{ Component } & \multicolumn{2}{|c|}{ Final concentration } \\
\hline 1 & $\mu \mathrm{L}$ & $\mathrm{KCl}(1 \mathrm{M}$ in water) & $*$ & 5 & $\mathrm{mM}$ \\
\hline 15 & $\mu \mathrm{L}$ & $\mathrm{MgCl}_{2}(1 \mathrm{M}$ in water) & $*$ & 75 & $\mathrm{mM}$ \\
\hline 1 & $\mu \mathrm{L}$ & PEP (100 mM in water) & $*$ & 0.8 & $\mathrm{mM}$ \\
\hline 4 & $\mu \mathrm{L}$ & PK $(2.5 \mathrm{mg} / \mathrm{mL}$ in buffer $)$ & $*$ & 50 & $\mu \mathrm{g} / \mathrm{mL}$ \\
\hline 4 & $\mu \mathrm{L}$ & LDH $(2.5 \mathrm{mg} / \mathrm{mL}$ in buffer $)$ & * & 50 & $\mu \mathrm{g} / \mathrm{mL}$ \\
\hline 1 & $\mu \mathrm{L}$ & UDP-Glc ( $1 \mathrm{M}$ in buffer) & $*$ & 5 & $\mathrm{mM}$ \\
\hline 1.2 & $\mu \mathrm{L}$ & NADH ( $25 \mathrm{mM}$ in buffer) & $*$ & 150 & $\mu \mathrm{M}$ \\
\hline $5-30$ & $\mu \mathrm{g}$ & UGT & & $50-150$ & $\mu \mathrm{g} / \mathrm{mL}$ \\
\hline $2-90$ & $\mu \mathrm{L}$ & Substrate ( $1 \mathrm{mM}$ in buffer) & & $10-450$ & $\mu \mathrm{M}$ \\
\hline 0.26 & $\mathrm{mg}$ & BSA (only for UGT76E1) & $*$ & 0.13 & $\%[\mathrm{w} / \mathrm{v}]$ \\
\hline $\mathrm{X}$ & $\mu \mathrm{L}$ & Gel filtration buffer & & & \\
\hline 200 & $\mu \mathrm{L}$ & Final volume & & & \\
\hline
\end{tabular}
UGT. Bovine serum albumin, BSA. Components marked with asterisks were mixed in a 2x master mix. 


\subsubsection{SUBSTRATE SYNTHESIS}

\subsubsection{ENZYMATIC SYNTHESES OF 9- AND 13-HYDROXY-OCTADECATRIENOIC ACIDS}

(10E,12Z,15Z)-9-Hydroxy-10,12,15-octadecatrienoic acid (9-HOT) and (9Z,11E,15Z)-13hydroxy-9,11,15-octadecatrienoic acid (13-HOT) were used as substrates for the UGT reactions. Both compounds were synthesized enzymatically and purified to homogeneity afterwards by Sabine Freitag (University of Goettingen, Germany).

In case of 9-HOT, the LOX from Solanum tuberosum was expressed from pET3b in BL21 Star (DE3) like described in Andreou et al., 2009. The bacterial cell pellet of $10 \mathrm{~mL}$ culture was used for the synthesis of 9-HOT. The pellet was resuspended in $1 \mathrm{~mL}$ lysis buffer $(50 \mathrm{mM}$ Tris/HCl $\mathrm{pH} 7.5,300 \mathrm{mM} \mathrm{NaCl}, 10 \%$ glycerol) supplemented with $1 \mu \mathrm{L}$ Tween 20 . The suspension was lysed by sonication as described above (see 3.2.5.1). The cell debris was sedimented by centrifugation at $3220 \mathrm{xg}$ and $4{ }^{\circ} \mathrm{C}$ for $4 \mathrm{~min} .4 \mathrm{~mL}$ of the crude lysate were mixed with $6 \mathrm{~mL}$ reaction buffer $(100 \mathrm{mM}$ Tris/ $\mathrm{HCl}, \mathrm{pH} 7.5)$ and $60 \mu \mathrm{L}$ linolenic acid $(250 \mathrm{mg} / \mathrm{mL})$. The reaction was incubated stirring on ice for $30 \mathrm{~min}$. Noticeable, every $5 \mathrm{~min}$, fresh air was bubbled into the solution with a Pasteur pipette. The reaction was determined by adding $10 \mathrm{~mL} \mathrm{SnCl}_{2}$ solution $(0.375 \%[\mathrm{w} / \mathrm{v}]$ in methanol) and incubating at room temperature for $10 \mathrm{~min}$. Likewise, the hydroperoxide products were reduced to the respective hydroxides. 9-HOT was extracted similar to Bligh \& Dyer, 1959: $100 \mu \mathrm{L}$ concentrated acetic acid and $10 \mathrm{~mL}$ chloroform were added to the reduced reaction solution of the LOX from Solanum tuberosum. The solution was vigorously mixed and centrifuged at $3220 \mathrm{x}$ g for $10 \mathrm{~min}$. The lower phase was transferred to a new reaction tube and the remaining solution was extracted with chloroform again. The second lower phase was combined with the first phase and the whole volume was evaporated under a stream of nitrogen. The residue was dissolved in $500 \mu \mathrm{L}$ ethanol.

In case of 13-HOT, the LOX from G. $\max$ (GmLOX, Merck KGaA, Darmstadt, Germany) was used to produce the compound. $100 \mu \mathrm{L}$ of $\mathrm{GmLOX}(0.250 \mathrm{mg} / \mathrm{mL})$ were mixed with $2 \mathrm{~mL}$ borate buffer ( $200 \mathrm{mM}$ borate, $\mathrm{pH}$ 9) and $60 \mu \mathrm{L}$ linolenic acid $(250 \mathrm{mg} / \mathrm{mL})$ and incubated in an open vessel at room temperature for $30 \mathrm{~min}$. Here, the reaction is stopped by adding appr. $0.1 \mathrm{~g}$ sodiumborhydride for reduction and proteins were precipitated by adding $100 \mu \mathrm{L}$ concentrated acetic acid.

\subsubsection{PURIFICATION OF 9- AND 13-HOT}

After synthesis and extraction, the compounds 9- and 13-HOT were purified by HPLC by S. Freitag (University of Goettingen, Germany). Both were purified by straight-phase HPLC SP HPLC: isocratic conditions with $0.8 \mathrm{~mL} / \mathrm{min}$ in hexane : isopropanole : trifluoroacetic acid 
[100: $1: 0.02, \mathrm{v} / \mathrm{v} / \mathrm{v}]$ on a ZORBAX RX-SIL $(4.6 \times 50 \mathrm{~mm}, 1.8 \mu \mathrm{m}$ particle size, Agilent, Waldbronn, Germany). The elution of 9-HOT and 13-HOT was monitored by $234 \mathrm{~nm}$ and their identity confirmed by by coelution with the authentic standards. The concentration of 9-HOT and 13-HOT were determined with the NanoDrop 2000 spectrophotometer (Thermo Fisher Scientific, Waltham, USA) at $234 \mathrm{~nm}$. The shared molar extinction coefficient of $23000 \mathrm{M}^{-1} \mathrm{~cm}^{-1}$ was used to calculate the concentration of 9-HOT and 13-HOT by the Lambert-Beer law (Equation 4).

\subsubsection{METABOLITE ANALYSES}

\subsubsection{HOMOGENIZING PLANT MATERIAL}

Plant material was homogenized before analyses. Therefore, two techniques were used: (i) for extracting DNA or RNA, one leaf was collected in a reaction tube and flash-frozen in liquid $\mathrm{N}_{2}$. The frozen tissue was grinded with a small pistil in the tube. (ii) Samples from a wounding experiment were homogenized using a Mixer Ball Mill MM200 (Retsch GmbH, Haan, Germany) at $30 \mathrm{~s}^{-1}$ for $120 \mathrm{~s}$. The device and the samples were constantly cooled with liquid $\mathrm{N}_{2}$.

\subsubsection{EXTRACTION OF PLANT MATERIAL}

The quantification of the plant hormones and, in particular jasmonates was essential to investigate their function in vivo. Here, a two phased methyl tert-butyl ether (MTBE) extraction was modified from Matyash et al., 2008. Briefly, appr. $100 \mathrm{mg}$ of the frozen tissue powder were weighted into $750 \mu \mathrm{L}$ methanol (LC-MS grade, MeOH). $2.5 \mathrm{~mL}$ MTBE and $80 \mu \mathrm{L}$ of an internal standard mixture (Table 3-28) were added and the solution was shaken in the dark at $4{ }^{\circ} \mathrm{C}$ and $200 \mathrm{rpm}$ for $1 \mathrm{~h} .600 \mu \mathrm{L}$ water were added for phase separation and mixed roughly before centrifugation at $450 \mathrm{x} g$ for $10 \mathrm{~min}$ at $22^{\circ} \mathrm{C}$. Then, the upper phase was taken and transferred to another glass tube.

\section{Table 3-28: Internal standards for quantitative LC-MS/MS analysis}

For quantification, internal standards were added to the extraction solutions. Five-fold deuterated 12 oxophytodienoic acid, $\mathrm{D}_{5}-12-\mathrm{OPDA}$; five-fold deuterated jasmonic acid; $\mathrm{D}_{5}-\mathrm{JA}$; four-fold deuterated jasmonoyl-leucine, $\mathrm{D}_{4}$-JA-Leu; four-fold deuterated salicylic acid, $\mathrm{D}_{4}$-SA; five-fold deuterated indole-3acetic acid, $\mathrm{D}_{5}$-IAA; six-fold deuterated abcisic acid, $\mathrm{D}_{6}-\mathrm{ABA}$; 2-oxothiazolidine-4-carboxylic acid (StRA).

\begin{tabular}{lrc}
\hline Substance & (concentration) & \multicolumn{1}{l}{ Volume } \\
\hline$D_{5}-12-O P D A$ & $(3 \mathrm{ng} / \mu \mathrm{L})$ & $10 \mu \mathrm{L}$ \\
D $_{5}$-JA & $(1 \mathrm{ng} / \mu \mathrm{L})$ & $10 \mu \mathrm{L}$ \\
D $_{3}$-JA-Leu & $(1 \mathrm{ng} / \mu \mathrm{L})$ & $20 \mu \mathrm{L}$ \\
D $_{6}$-SA & $(1 \mathrm{ng} / \mu \mathrm{L})$ & $10 \mu \mathrm{L}$ \\
D $_{5}$-IAA & $(1 \mathrm{ng} / \mu \mathrm{L})$ & $10 \mu \mathrm{L}$ \\
D $_{6}$-ABA & $(1 \mathrm{ng} / \mu \mathrm{L})$ & $10 \mu \mathrm{L}$ \\
St-RA & $(5 \mathrm{ng} / \mu \mathrm{L})$ & $10 \mu \mathrm{L}$ \\
\hline
\end{tabular}


$700 \mu \mathrm{L} \mathrm{MeOH}$ : water $[3: 2.5, \mathrm{v} / \mathrm{v}]$ and $1.3 \mathrm{~mL}$ MTBE were mixed to the remaining phase for reextraction. After centrifugation as before, the upper and the lower phase were taken and pooled with the first upper phase. This solution was evaporated under a stream of nitrogen. The residue was dissolved in $100 \mu \mathrm{L} \mathrm{MeOH}$ twice and transferred to a reaction tube. For LC-MS measurements, the extracts were evaporated again and resolved in $20 \mu \mathrm{L}$ solvent B (ACN : water, [90: 10, v/v], $\left.0.3 \mathrm{mM} \mathrm{NH}_{4} \mathrm{COOH}, \mathrm{pH} 3.5\right)$ first. Then, $80 \mu \mathrm{L}$ solvent $\mathrm{A}\left(0.3 \mathrm{mM} \mathrm{NH} \mathrm{COOH}_{4}\right.$ in water, $\mathrm{pH} 3.5$ ) were added to fit to the starting conditions of the chromatographic separation by LC.

\subsubsection{TARGETED LC-MS/MS ANALYSIS OF PHYTOHORMONES}

The LC-MS/MS measurement of the phytohormones were performed by Dr. Cornelia Herrfurth and Dr. Krzysztof Zienkiewicz (both University of Goettingen, Germany). The extracts were analyzed by ultra-perfomance-LC (UPLC) nano-electrospray ionization (ESI) MS-fragmentation (MS/MS) method according to Ghareeb et al., 2011. An Acquity UPLC system equipped with ACQUITY UPLC HSS T3 column $(100 \mathrm{~mm}$ x $1 \mathrm{~mm}, 1.8 \mu \mathrm{m}$ particle size; both from Waters Corp., Milford, MA, USA) was coupled to a nanoESI chip ion source (TriVersa NanoMate; Advion BioSciences, Ithaca, USA). The MS analysis was performed with an Applied Biosystems 4000 quadrupole/linear ion trap mass spectrometer (MDS Sciex, Ontario, Canada). For analysis, $10 \mu \mathrm{L}$ of the extract were injected (see 3.2.8.2). The binary gradient system consisted of solvent $\mathrm{A}$, water and solvent $\mathrm{B}, \mathrm{ACN}$ : water, $90: 10$ [v/v], both containing $0.3 \mathrm{mM} \mathrm{NH}_{4} \mathrm{COOH}$ (adjusted to $\mathrm{pH} 3.5$ with formic acid) was used. Elution was performed with the following gradient program: $10 \%$ solvent B for 0.5 min, followed by a linear increase of solvent B up to $40 \%$ within 1.5 min held for 2 min and followed by increase of solvent B up to $95 \%$ solvent B in 1 min. An isocratic run at $95 \%$ solvent $\mathrm{B}$ was then held for $2.5 \mathrm{~min}$. To recover starting conditions a linear decrease to $10 \% \mathrm{~B}$ within $3 \mathrm{~min}$ was performed. The flow rate was $0.16 \mathrm{~mL} / \mathrm{min}$. For stable nanoESI conditions, $70 \mu \mathrm{L} / \mathrm{min}$ of 2 -propanol/ACN/water $[70: 20: 10, \mathrm{v} / \mathrm{v} / \mathrm{v} /]$ containing $0.3 \mathrm{mM} \mathrm{NH}_{4} \mathrm{HCOO}$ (adjusted to $\mathrm{pH} 3.5$ with formic acid) delivered by a Pharmacia 2248 HPLC pump (GE Healthcare, Munich, Germany) were added just after the column via a mixing tee valve. By using a second post column splitter $502 \mathrm{nl} / \mathrm{min}$ of the eluent were directed to the nanoESI chip with $5 \mu \mathrm{m}$ internal diameter nozzles. Ionization voltage was set to $-1.7 \mathrm{kV}$, phytohormones were negatively ionized and detected in a scheduled multiple reaction monitoring (MRM) mode with AB Sciex 4000 QTRAP tandem mass spectrometer (AB Sciex, Framingham, MA, USA). For the scheduled mode, the MRM detection window was $72 \mathrm{~s}$ and a target scan time of $1.2 \mathrm{~s}$ was set. Mass transitions were optimized for every compound individually (Table 3-29). The mass analyzers were adjusted to a resolution of $0.7 \mathrm{amu}$ full width at half height. The ion source temperature was $40^{\circ} \mathrm{C}$ and the curtain gas was set at 10 - given in arbitrary units. Quantification was carried out using a calibration curve of intensity relations of the mass-to- 
charge ratio $(\mathrm{m} / \mathrm{z})$ of standard substances vs. molar amounts of unlabeled metabolites (0.3 - 1000 pmol, [unlabeled]/[deuterium-labeled]).

Table 3-29: Transitions of the multiple reaction monitoring mode of the LC-MS/MS analysis Metabolite in the LC-MS/MS analysis were identified in the multiple reaction monitoring mode by selecting their mother ion and an fragment for quantification. Every fragmentation scan has its individual clustering potential (DP), entrance potential (EP), and collision energy (CE). 12-Oxophytodienoic acid, 12-OPDA; jasmonic acid, JA; 11/12-hydroxy-JA, 11/12-OH-JA; 12-hydroxy-jasmonoyl sulfate, 12 $\mathrm{HSO}_{4}$-JA; 12 -glycosyl-O-JA, 12-O-Glc-JA; jasmonoyl-isoleucine/leucine, JA-Ile/Leu; 12-hydroxy-JAIle, 12-OH-JA-Ile; 12-carboxy-JA-Ile, 12-COOH-JA-Ile, abscisic acid, ABA; ABA-glycosyl ester, ABAGE; salicylic acid, SA; 2-O-glycosyl-SA, 2-O-Glc-SA; indole-3-carboylic acid, ICA; indole-3-acetic acid, IAA; five-fold deuterated 12-oxophytodienoic acid, $\mathrm{D}_{5}-12-\mathrm{OPDA}$; five-fold deuterated jasmonic acid; $\mathrm{D}_{5}$ $\mathrm{JA}$; four-fold deuterated jasmonoyl-leucine, $\mathrm{D}_{4}$-JA-Leu; four-fold deuterated salicylic acid, $\mathrm{D}_{4}$-SA; fivefold deuterated indole-3-acetic acid, $\mathrm{D}_{5}$-IAA; six-fold deuterated abcisic acid, $\mathrm{D}_{6}$-ABA.

\begin{tabular}{|c|c|c|c|c|c|}
\hline Q1 & Q3 & Analyte & $\mathrm{DP}(\mathrm{V})$ & EP (V) & $\mathrm{CE}(\mathrm{eV})$ \\
\hline 291 & 165 & 12-OPDA & -70 & -8.5 & -28 \\
\hline 209 & 59 & JA & -30 & -4.5 & -24 \\
\hline 225 & 59 & 11/12-OH-JA & -35 & -9.0 & -28 \\
\hline 305 & 97 & $12-\mathrm{HSO}_{4}-\mathrm{JA}$ & -30 & -4.0 & -32 \\
\hline 387 & 59 & Glc- $O$-JA & -85 & -9.0 & -52 \\
\hline 322 & 130 & JA-Ile/Leu & -45 & -5.0 & -28 \\
\hline 352 & 130 & 12-COOH-JA-Ile & -45 & -10.0 & -30 \\
\hline 338 & 130 & 12-OH-JA-Ile & -45 & -10.0 & -30 \\
\hline 263 & 153 & $\mathrm{ABA}$ & -35 & -4.0 & -14 \\
\hline 425 & 263 & ABA-GE & -30 & -10.0 & -16 \\
\hline 137 & 93 & SA & -25 & -6.0 & -20 \\
\hline 299 & 137 & 2-O-Glc-SA & -30 & -4.0 & -18 \\
\hline 160 & 113 & ICA & -40 & -6.5 & -22 \\
\hline 174 & 130 & IAA & -35 & -9.0 & -14 \\
\hline 396 & 107 & $\mathrm{D}_{5}-12-\mathrm{OPDA}$ & -65 & -4.0 & -28 \\
\hline 214 & 62 & $\mathrm{D}_{6}-\mathrm{JA}$ & -35 & -8.5 & -24 \\
\hline 325 & 133 & $\mathrm{D}_{4}$-JA-Leu & -80 & -4.0 & -30 \\
\hline 141 & 97 & $\mathrm{D}_{4}-\mathrm{SA}$ & -25 & -6.0 & -22 \\
\hline 153 & 109 & $\mathrm{D}_{5}$-IAA & -50 & -4.0 & -22 \\
\hline 293 & 179 & $\mathrm{D}_{6}-\mathrm{ABA}$ & -80 & -10.0 & -42 \\
\hline
\end{tabular}

\subsubsection{METABOLITE ANALYSIS BY LC-HIGH RESOLUTION-MS}

The metabolite fingerprinting experiments were performed by LC-high resolution-MS (LC-HRMS) together with Dr. Kirstin Feussner (University of Goettingen, Germany) according to Nahlik et al., 2010. The samples were separated by an Acquity UPLC system with UPLC eLambda 800 nm PDA detector and equipped with an Acquity UPLC BEH SHIELD RP18 column (1x100 mM, $1.7 \mu \mathrm{m}$ particle size, Waters Corporation, Milford, USA). A flow rate of $0.2 \mathrm{ml} \mathrm{min}^{-1}$ at $40{ }^{\circ} \mathrm{C}$ was applied. Separation was gained with a binary gradient of solvent A (water : formic acid $[100: 0.1, \mathrm{v} / \mathrm{v}]$ and solvent $\mathrm{B}(\mathrm{ACN}:$ formic acid $[100: 0.1, \mathrm{v} / \mathrm{v}]$. The run was set with $0-0.5$ min at $10 \%$ solvent B, $0.5-3$ min gradual to $28 \%$ solvent B, $3-8$ min gradual to $95 \%$ solvent B, 8-10 min at $95 \%$ solvent B, and $10-14 \min 10 \%$ solvent. The LC-HR-MS was operated in negative ESI mode with W optics to ensure a mass resolution larger than 10000 . Data were acquired by MassLynx 4.1 software in centroid format over a mass range of $m / z 85-1200$ 
with a scan duration of $0.5 \mathrm{~s}$ and an interscan delay of $0.1 \mathrm{~s}$. The capillary and the cone voltage were maintained at $2600 \mathrm{~V}$ and $30 \mathrm{~V}$ and the desolvation and source temperature at $350{ }^{\circ} \mathrm{C}$ and $80{ }^{\circ} \mathrm{C}$ respectively. Nitrogen was used as cone $\left(30 \mathrm{~L} \mathrm{~h}^{-1}\right)$ and desolvation gas $\left(800 \mathrm{~L} \mathrm{~h}^{-1}\right)$. The Dynamic Range Enhancement mode was used for data recording. All analyses were monitored by using Leucine-enkephaline $\left([\mathrm{M}-\mathrm{H}]^{-} 554.2615\right.$ or $[\mathrm{M}+\mathrm{H}]^{+} 556.2771$ as well as its ${ }^{13} \mathrm{C}$ isotopomer $[\mathrm{M}-\mathrm{H}]^{-} 555.2615$ or its double ${ }^{13} \mathrm{C}$ isotopomer $[\mathrm{M}+\mathrm{H}]^{+} 558.2836$ as lock spray reference compound at a concentration of $0.5 \mu \mathrm{g} \mathrm{mL} \mathrm{m}^{-1}$ in $\mathrm{ACN}$ : water $[50: 50, \mathrm{v} / \mathrm{v}]$ and a flow rate of $30 \mu 1 \mathrm{~min}^{-1}$. For targeted data analysis, the MassLynx 4.1 software was used to investigate metabolites.

\subsubsection{NON-TARGETED EX VIVO METABOLITE FINGERPRINTING}

The non-targeted ex vivo experiments were performed together with Dr. Kirstin Feussner (GeorgAugust-University, Goettingen, Germany). It uses the metabolite fingerprinting platform (Kaever et al., 2014) and connects it to activity assays with purified enzymes. The purified enzyme were incubated with an enriched metabolite extracts from both phases of the MTBE-extraction (see 3.2.8.2). Here, the plant material of a wounding experiment (see 3.2.1.3) was used to provide the enzyme with the enriched metabolite extract including wound-accumulated metabolites like 12OH-JA. The reactions were measured by the non-targeted fingerprinting pipeline (Kaever et al., 2014). The activity assays were performed as following: $100 \mu \mathrm{g}$ homogeneous protein were incubated with $83 \mu \mathrm{L}$ enriched plant extract (of $100 \mu \mathrm{L}$ from $120 \mathrm{mg}$ plant material), and $10 \mu \mathrm{M}$ UDP-Glc. As positive control, known substrates were spiked into a selected number of reactions: $10 \mu \mathrm{M}$ 12-OH-JA, $10 \mu \mathrm{M} \mathrm{SA}$, and $10 \mu \mathrm{M} \omega-\mathrm{OH}-16: 0$. The reactions were incubated at $25{ }^{\circ} \mathrm{C}$ for $1 \mathrm{~h}$ and terminated by adding $20 \mu \mathrm{L} \mathrm{ACN}$. For measurement, the solutions were centrifuged at $20000 \mathrm{x} \mathrm{g}$ at $4{ }^{\circ} \mathrm{C}$ for $10 \mathrm{~min}$ and $70 \mu \mathrm{L}$ were transferred into UPLC-vials. The non-targeted $e x$ vivo activity assay was performed as three independent experiments which were performed at the same time, treated equally and measured together.

The technical parameters of the LC-HR-MS were set as described in 3.2.8.4. Samples were measured in positive and negative ionization mode. For data processing, the raw data of all samples of one experiment were taken and processed with MarkerLynx Application Manager for MassLynx 4.1 software resulting in two data matrices - from the two ionization modes. The toolbox MarVis (MarkerVisualization, http://marvis.gobics.de) was used for further data processing including ranking by ANOVA combined with the multiple testing by BenjaminiHochberg, filtering the data, adduct and isotope correction as well as combining of the selected data of the two data matrixes (MarVis Filter) for clustering and visualization (MarVis Cluster). For database search, metabolite set enrichment analyses (MarVis Pathway) were used. Here, also internal databases optimized for $e . g$. jasmonates and their glucosides were integrated. Selected high quality features (false discovery rate $<10^{-6}$ ) were chosen for clustering and data base search. 
The identity of selected features was confirmed by coelution with authentic standards and/or by fragmentation analysis.

\subsubsection{STRUCTURE DETERMINATION BY FRAGMENTATION}

Detailed structural information of different markers identified by metabolite fingerprinting were confirmed by fragmentation (MS/MS) using a LC 1290 Infinity (Agilent Technologies, Santa Clara, USA) coupled with an Applied Biosystems 6540 UHD Accurate-Mass triple quadrupole time of flight mass spectrometer (Agilent Technologies, Santa Clara, USA). The MS/MS analyses were done by Dr. Kirstin Feussner (Georg-August-University, Goettingen, Germany).

For LC an Acquity UPLC HSS T3 column $(1 \times 100 \mathrm{~mm}, 1.8 \mu \mathrm{m}$ particle size, Waters Corporation, Milford, USA) was used at a flow rate of $0.5 \mathrm{ml} \mathrm{min}^{-1}$ and a temperature of $40{ }^{\circ} \mathrm{C}$. The binary solvent system consists of A (water : formic acid [100:0.1, v/v] and B (ACN : formic acid [100:0.1, v/v]. The gradient was set as for LC-HR-MS analysis (3.2.8.4). The MSinstrument was operated with a detection frequency of $2 \mathrm{GHz}$ in the Extended Dynamic Range and the targeted MS/MS mode. The source conditions were set with gas temperature: $250{ }^{\circ} \mathrm{C}$, drying gas flow: $8 \mathrm{~L} \mathrm{~min}^{-1}$, nebulizer pressure: $35 \mathrm{psi}$, sheath gas temperature: $300{ }^{\circ} \mathrm{C}$, sheath gas flow: $8 \mathrm{~L} \mathrm{~min}^{-1}$, VCap voltage: $3 \mathrm{kV}$, nozzle voltage: $200 \mathrm{~V}$, and fragmentor voltage: $100 \mathrm{~V}$. Samples were ionized in negative and/or positive ESI mode with collision energy 12-32 eV. Isolation of precursor ions occurred within the narrow isolation width of $1.3 \mathrm{~m} / \mathrm{z}$. Data were acquired by Mass Hunter Workstation Acquisition software B.05.01 (Agilent Technologies, Santa Clara, USA). Mass Hunter Qualitative Analysis B.06.01 (Agilent Technologies, Santa Clara, USA) was used for data analysis. 


\section{RESUlTS}

The JA-pathway has been studied intensively (Koo, 2018; Wasternack, 2015) and many of the enzymes of the JA-Ile-synthesis as well as for its catabolism have been described (see Figure 1-3). Up to now, the enzyme(s) responsible for the glycosylation of the 12-hydroxy group of 12-OHJA are still elusive although the metabolite 12-O-Glc-JA is known in A. thaliana (Koo, 2018; Miersch et al., 2008).

Here, four uncharacterized UGTs are investigated towards their activity on jasmonates - in particular towards 12-OH-JA. The work was divided into several subtopics: (i) the identification of potential candidate $U G T$ s and (ii) their expression analysis in wounded A. thaliana. Then, (iii) the candidate enzymes were characterized biochemically in respect to their preferred substrate and their kinetic parameters. On top of this, (iv) the in vivo analysis of the candidates was started. Therefore, the CRISPR/Cas9 approach was used to knock out the candidate genes specifically.

\subsection{IDENTIFICATION OF FOUR UGTS AS CANDIDATES FOR 12-OH-JA GLYCOSYLATION}

After wounding, the modifications within the JA metabolic fate follow a time-course (see 4.4). This temporal distribution is reflected in the expression of such genes, too. Likewise, it may be possible to identify genes involved in the glycosylation of 12-OH-JA by co-expression analysis of gene products, which are known to synthetize and metabolize 12-OH-JA. The UGT family of A. thaliana has been studied phylogenetically before (Lim et al., 2002; Ross et al., 2001) and, hence, the putative genes connected to jasmonates are annotated and can be identified by online searches.

\subsubsection{UGT76E1 AND UGT76E2 MAY BE RELATED TO 12-OH-JA METABOLISM}

12-OH-JA is directly oxidized from JA by JOX 1 to JOX 4 (Caarls et al., 2017). Therefore, the gene products of $J O X 1$ to $J O X 4$ were used as bait genes for RNA-seq co-expression analyses. For $J O X 1$, JOX2, and JOX4, no co-regulations with predicted UGTs were found (data not shown). Whereas, JOX3 shows co-expression with its homologue JOX4, At2g39030, At4g37990, At1g52890, At2g36080, and, interestingly, with At5g59580 encoding for UGT76E1 (Table 4-1). $U G T 76 E 1$ is a so far uncharacterized $U G T$. Searching against expression data from micro-array analysis, JOX3 is co-regulated with other members in the JA-metabolism CYP94B3, ILL6, TIFY7, TIFY 9, and TIFY 10 (Supplemental figure 2A) but not with UGT76E1 here. Next, ST2a, which's 
gene product forms 12- $\mathrm{HSO}_{4}-\mathrm{JA}$ from 12-OH-JA, was used as a bait gene for the RNA-seq data. No co-expression is shown for known players around jasmonates. Anyhow, ST2a is co-expressed with At5g59590 encoding for UGT76E2 (Table 4-1) - a second uncharacterized UGT. Further, the available micro-array data of $S T 2 a$ were evaluated but no co-regulations with UGT76E2 or other putative UGTs were given (Supplemental figure 2B).

Furthermore, gene products, which are known to be involved in the jasmonate inactivation were investigated. ILL6 and IAR3, whose gene products produce 12-OH-JA by de-conjugating 12-OHJA-Ile, did not show co-regulation with putative UGTs (data not shown). Additionally, CYP94B1, CYP94B3, and CYP94C1 oxidizing JA-Ile to 12-OH-JA-Ile were used as baits for co-expression searches. The 12-hydroxy group could be a target for further glycosylation to potentially form 12 Glc-O-JA-Ile. However, no co-expressions of putative UGTs were found with the JA-Ile oxidizing P450s as baits (data not shown).

Taken together, the search for gene products involved in the jasmonate catabolism identified two uncharacterized $U G T \mathrm{~s}$ as candidates for the glycosylation of 12-OH-JA: UGT76E1 and UGT76E2.

\section{Table 4-1: Search for glycosyltransferase genes as candidates for JA-glycosylation}

RNA-seq co-expression analysis of gene products involved in 12-hydroxy-JA metabolism. Jasmonic acid oxidase $3(\mathrm{JOX} 3$, At3g55970) and sulfotransferase 2a (ST2a, At5g07010) were used as bait genes to search for candidates for JA-glycosylation. Two uncharacterized glycosyltransferases, UGT76E1 and UGT76E2, were identified (red frame). Given are the complete outputs of the data base search for every bait. The gene products are ranked by Mutual Rank (MR) giving an average correlation of two genes. Smaller MR-values indicate stronger correlation. Access 02.05.2018; the page was prepared on Dec. 14. 2017 for ATTED-II version 9.1

\begin{tabular}{|c|c|c|}
\hline MR & Locus & Function \\
\hline \multicolumn{3}{|c|}{ Bait gene: JOX3 (At3g55970) } \\
\hline 1.6 & At2g38240 & $\begin{array}{l}\text { 2-Oxoglutarate and Fe(II)-dependent oxygenase Superfamily protein, } \\
\text { JOX } 4\end{array}$ \\
\hline 1.8 & At $2 \mathrm{~g} 39030$ & Acyl-CoA N-acyltransferases superfamily protein \\
\hline 3.2 & At4g37990 & Elicitor-activated gene $3-2$ \\
\hline 3.4 & Atlg52890 & NAC domain containing protein 19 \\
\hline 18.9 & At5g59580 & UGT76E1 \\
\hline 33.4 & At2g36080 & AP2/B3-like transcriptional factor family protein \\
\hline \multicolumn{3}{|c|}{ Bait gene: $S T 2 a(A t 5 g 07010)$} \\
\hline 7.7 & Atlg54100 & Aldehyde dehydrogenase 7B4 \\
\hline 19.3 & At2g38240 & $\begin{array}{l}\text { 2-Oxoglutarate and Fe(II)-dependent oxygenase Superfamily protein, } \\
\text { JOX } 4\end{array}$ \\
\hline 20.9 & Atlg64660 & Methionine gamma-lyase \\
\hline 37.1 & At4g31380 & FPF1-like protein 1 \\
\hline 44.1 & At5g59590 & UGT76E2 \\
\hline 50.9 & Atlg75450 & Cytokinin oxidase 5 \\
\hline
\end{tabular}




\subsubsection{TRANSCRIPTOME ANALYSIS OF WOUNDED $A$. THALIANA LEAVES CONFIRMED $U G T 76 E 1, U G T 76 E 2$ AND IDENTIFIED $U G T 76 E 11$ AND UGT76E12 AS WOUND INDUCED TRANSCRIPTS}

A second source was used to support the candidates UGT76E1 and UGT76E2 and to search for additional $U G T \mathrm{~s}$, which could be involved in the catabolism of 12-OH-JA. Therefore, a combined transcriptome and metabolome study of a wounding time course for A. thaliana leaves was used (Kaever, 2014). The data were recorded $0,0.5$ and $2 \mathrm{hpw}$ in leaves of wild type Col-0 and the JAdeficient mutant delayed dehiscence 2-2 (dde2-2) (Park et al., 2002). This mutant is a loss-offunction point mutation in the $A O S$, which results in JA-deficient plants. The transcriptome data were recorded by a $44 \mathrm{k}$ Affimetrix micro-array analysis.

There, the candidates $U G T 76 E 1$ and $U G T 76 E 2$ showed detectable signals at 2 hpw exclusively; all other time points and the $d d e 2-2$ show just trace signals. Yet, the intensity of UGT76E2 is below the signal threshold (> 500 procedure defined units (p.d.u.), oral communication Milena Lewandowska, University of Goettingen, Germany). In addition, a $U G T$ related screening ( $c f$. Figure 4-2) identified UGT76E11 (At3g46670) and UGT76E12 (At3g46660). UGT76E11 shows constitutive expression with additional induction at $2 \mathrm{hpw}$ in Col-0 (5-fold) and $d d e 2$-2 (2-fold). Similar to UGT76E1 and UGT76E2, UGT76E12 displays wound induction in Col-0 at $2 \mathrm{hpw}$ exclusively but with 5-fold higher intensities (Supplemental figure 3). Altogether, UGT76E1 and UGT76E2 show low signal intensities around the signal threshold whereas UGT76E12 depicts robust induction. The accumulations of UGT76E1, UGT76E2, and UGT76E12 at $2 \mathrm{hpw}$ are strongly JA-dependent and the reduced expression of UGT76E11 in dde2-2 at $2 \mathrm{hpw}$ suggest a JA-influence.

The Arabidopsis Information Resource provides another source of expression data. There, the online tool Arabidopsis eFP Broswer offers transcriptome data for various stimuli, tissues, or developmental stages (Winter et al., 2007). In this context, focus was given to the expression of the candidate UGTs after abiotic stress like wounding (Kilian et al., 2007), cold, osmotic, salt, drought, UV-B, and heat stress.

UGT76E1 is exclusively expressed in A. thaliana shoots in response to wounding (1 hpw, Supplemental figure 4A). UGT76E2 displays also a specific expression $1 \mathrm{hpw}$ in shoots and, in addition, a constitutive expression in roots (Supplemental figure 4B). The expression of UGT76E1 and UGT76E2 is not induced by other kinds of abiotic stress like cold, osmotic, salt, drought, UV-B, and heat stress. In contrast to that, UGT76E11 shows constitutive expression in shoots, which could be enhanced mainly by cold ( 3 hours after all stimulus) and salt stress (12 hours after all stimuli) but no expression in roots. Interestingly, in response to wounding, the expression of $U G T 76 E 11$ is reduced at $0.5 \mathrm{hpw}$ similar to the results of the in-house transcriptome analysis shown above (Supplemental figure 5A, see Supplemental figure 3C) and started to 
increase $1 \mathrm{hpw}$. UGT76E12 illustrated specific expressions at $1 \mathrm{hpw}, 24 \mathrm{~h}$ after oxidative stress, and highest inductions at $24 \mathrm{~h}$ after osmotic stress in shoots. No expression of UGT76E12 is depicted in roots (Supplemental figure 5B). The expression data of the Arabidopsis eFP Broswer highlighted differential expression of UGT76E1 and UGT76E2 in roots and confirmed the findings in respect to the wound induction of the four candidate genes.

\subsubsection{REAL TIME PCR ANALYSES CONFIRM, THAT UGT76E1, UGT7E11, AND UGT76E12 ARE INDUCED AFTER WOUNDING}

To support the data of the co-expression analyses and transcriptome array, UGT76E1, UGT76E2, UGT76E11, and UGT76E12 were analyzed by a quantitative expression analysis performed by qPCR. The expression analysis was performed with cDNA, which had been synthesized from RNA of wounded leaves $(0,1,2$, and $5 \mathrm{hpw}$, see 4.4). Here, UGT74F1 (At2g43840) was added as control gene to the set of candidate genes: UGT74F1 encodes a glycosyltransferase, that specifically glycosylates SA (George Thompson et al., 2017; Lim et al., 2004) and acts as woundunrelated control here.

The obtained raw data were normalized to the basal expression of the respective genes at $0 \mathrm{hpw}$. At 1 hpw, UGT76E1 and UGT76E2 show slight elevation with 2-fold higher expression. While, UGT76E11 and UGT76E12 already show significant accumulation with 4-fold and 3.5-fold enrichment, respectively. At $2 \mathrm{hpw}$, the expression rates of UGT76E1, UGT76E11, and UGT76E12 accumulated significantly with about 6- to 8-fold higher expressions. Whereas, UGT76E2 shows no further accumulation. At $5 \mathrm{hpw}$, the expressions of UGT76E1 and UGT76E12 decreases to levels of UGT76E2 (2-fold higher expression), while UGT76E11 still displays 5.5-fold higher expression in comparison to the unwounded plants. The control, UGT74F1 shows no induction after wounding at any of the time points (Figure 4-1A). Without normalization, UGT76E1, UGT76E2, UGT76E11, and UGT74F1 display similar low constitutive expression levels. Only UGT76E12 shows 2-fold elevated levels without wounding stimulus (Supplemental figure 6A). Furthermore, the expressions were compared between Col-0 and $d d e 2$ 2 at 0 and 2 hpw. The relative expression values of UGT76E1, UGT76E2, and UGT76E12 show control-like levels in dde2-2 at $2 \mathrm{hpw}$ whereas UGT76E11 shows 3 -fold elevated levels (Supplemental figure 6B).

In summary, the quantitative expression analysis of the four candidate $U G T$ s illustrates that UGT76E1, UGT76E11, and UGT76E12 are significantly upregulated in Col-0 after wounding. In contrast to that, UGT76E2 accumulates slightly but not significantly after wounding. The results of the quantitative expression analysis confirm the expression pattern of the transcriptome analysis for $U G T 76 E 1, U G T 76 E 11$, and $U G T 76 E 12$, even though the relative expression rates 
and fold changes are different (see 4.1.2). Contradictory to the transcriptome data, UGT76E12 did not show significant accumulation after wounding.

So far, three different approaches - the co-expression analysis, the $44 \mathrm{k}$ Affimetrix transcriptome analysis of wounded A. thaliana leaves, and the quantitative expression analysis by qPCR identified UGT76E1, UGT76E2, UGT76E11, and UGT76E12 as related to the JA-mediated wound-response in A. thaliana, even if the results for the three data sources are not completely consistent.

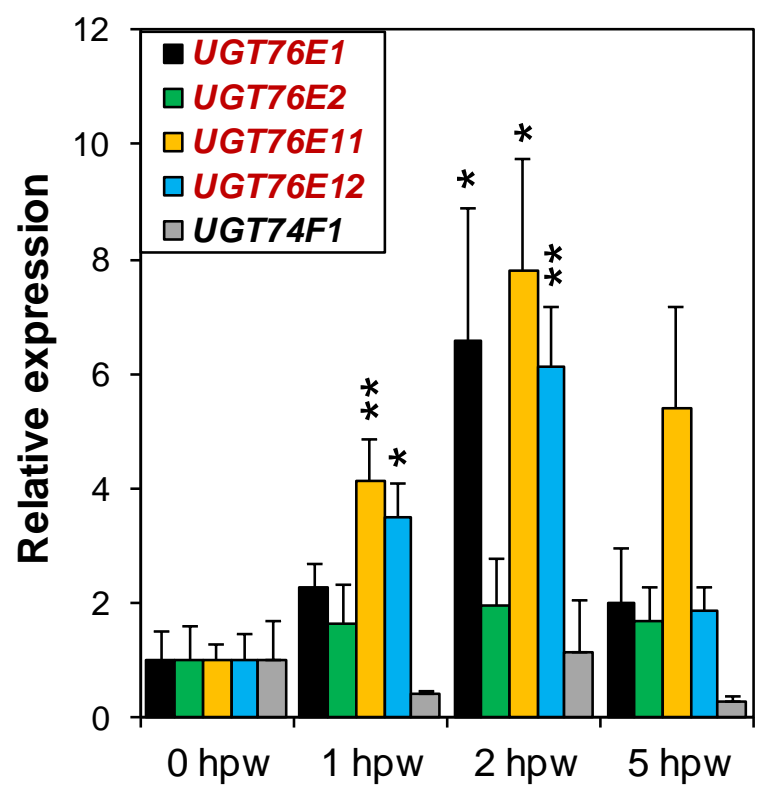

Figure 4-1: Expression of UGT76E1, UGT76E2, UGT76E11, UGT76E12, and UGT74F1 in A. thaliana leaves after wounding

Quantitative real-time PCR of UGT76E1 (red), UGT76E2 (green), UGT76E11 (orange), UGT76E12 (blue), and $U G T 74 F 1$ (grey). Plants were grown for six weeks at $22{ }^{\circ} \mathrm{C}$ under short day conditions $(8 \mathrm{~h}$ light/16 $\mathrm{h}$ dark). Leaves were wounded three times across the mid vein by squeezing with forceps. Damaged rosette leaves were harvested at indicated time points (hours post wounding (hpw)), RNA was isolated, and appr. $1 \mu \mathrm{g}$ transcribed complementary DNA were used for PCR. All expression values are normalized to actin 8 as reference. Relative expression of the transcripts normalized to their respective expression levels at $0 \mathrm{hpw}$. Each data point represents the mean value $+\mathrm{SD}$ of three biological replicates. 10 plants per time point were pooled for one replicate. Asterisks indicate significance by one-sided T-Test with $* \mathrm{p}<0.05, * * \mathrm{p}<.0 .01$

\subsubsection{UGT76E1 AND UGT76E2 ARE ASSIGNED TO JA METABOLISM BY CO- EXPRESSION ANALYSES}

UGT76E1, UGT76E2, UGT76E11, and UGT76E12 have been identified as candidates for the glycosylation of jasmonates by co-expression with known players in jasmonate metabolism. Now, the candidates were used as baits for co-expression analysis to support the connection to jasmonates from the other side. 
UGT76E1 displays connections to JAZ2, ILL6, and JOX3, which are members of the JA-mediated wound-response in A. thaliana (Table 4-2). UGT76E2 shows co-regulation with genes induced by osmotic stress (data not shown, eFP-Browser, access 02.05.2018) like cinnamyl alcohol dehydrogenase 5 ( $c f$. Lee et al., 2013), Myzus persicae-induced lipase 1 (cf. Louis et al., 2010) and UGT71B8, which glycosylates ABA (Dong et al., 2014). Furthermore, UGT76E2 is coregulated with ST2a, by which it also was identified before (Table 4-2). Next, UGT76E11 shows co-regulation with genes connected to drought response but not wounding. Like for UGT76E11 itself, the co-regulated genes are induced by different abiotic stimuli with generally high expressions (data not shown, eFP-Browser, access 02.05.2018). UGT76E12 is co-regulated with genes induced by osmotic stress (data not shown, eFP-Browser, access 02.05.2018) like elicitoractivated gene 3-2 (cf. Somssich et al., 1996), CYP76C2 (cf. Hofer et al., 2014), and phenolic lucoside malonyltransferase 1 ( $c f$. Khan et al., 2016). None of them is known to have functions in wounding.

Table 4-2: Co-expression analysis of UGT76E1, UGT76E2, UGT76E11, and UGT76E12 RNA-seq co-expression analysis of gene products correlated with UGT76E1 (At5g59580), UGT76E2 (At5g59590), UGT76E11 (At3g46670), and UGT76E12 (At3g46660). Identified gene products related to JA metabolism are given in bold. JAZ2, jasmonate-ZIM-domain protein 2; JOX3, jasmonic acid oxidase 3. Given are the complete outputs of the database for every bait. Gene products are ranked by Mutual Rank (MR) giving an average correlation of two genes indicating stronger correlation by smaller values. Access 02.05.2018; the page was prepared on Dec. 14. 2017 for ATTED-II version 9.1

\begin{tabular}{ccc}
\hline MR & Locus & Function \\
\hline & & Bait gene: UGT76E1 (At5g59580) \\
\hline 8.5 & At1g74950 & TIFY domain/Divergent CCT motif family protein, JAZ2 \\
14.4 & At1g44350 & IAA-leucine resistant (ILR)-like gene 6, ILL6 \\
Jasmonate-regulated gene 21, JOX3
\end{tabular}

In addition to that, the available micro-array data of UGT76E1, UGT76E2, UGT76E11, and UGT76E 12 were checked. Here, the data did not show direct evidence for a potential role of any candidate in the glycosylation of jasmonates (Supplemental figure 7, Supplemental figure 8). Interestingly, UGT76E12 is co-regulated with a gene encoding for $\beta$-glucosidase 11 (At1g02850) 
(Supplemental figure 8B). This gene is expressed late after wounding - $12 \mathrm{hpw}$ - but also after other abiotic stresses (data not shown, eFP-Browser, access 02.05.2018). Since $\beta$-glucosidases cleave glycosidic bonds, it might be connected to UGT76E12.

\subsection{SEQUENCE ANALYSES OF THE CANDIDATES}

\subsubsection{UGT76E1, UGT76E2, UGT76E11, AND UGT76E12 HAVE SIMILAR GENE STRUCTURES}

In the A. thaliana genome, the four candidate genes are found in two loci. UGT76E1 (AT5g59580) and UGT76E2 (At5g59590) are located right next to each other on the chromosome five. Similarly, UGT76E11 (At3g46670) and UGT76E12 (At3g46660) are also located side by side on the chromosome three. Allover, the gene structures of all four genes are similar: they have two exons split by one intron located at the first third of the genes (Supplemental figure 9). No splicevariants are known for the candidates. This is supported by findings that $U G T$ s are clustered in groups of two to seven genes and harbor none to two introns (Ross et al., 2001).

\subsubsection{UGT76E1 AND UGT76E2 AS WELL AS UGT76E11 AND UGT76E12 CLUSTER AS HOMOLOGUES}

As members of the UGT subfamily E, UGT76E1, UGT76E2, UGT76E11, and UGT76E12 are related with $\geq 60 \%$ amino acid sequence homology (Mackenzie et al., 1997). In the phylogenetic tree of all UGTs of A. thaliana, the four candidates for 12-OH-JA glycosylation cluster in the group $\mathrm{H}$, which contains all members of the UGT76 family. The tree was generated by comparing ten conserved motifs of all UGTs in A.thaliana (Figure 4-2, see Supplemental figure 1). Therefore, the candidates share $>60 \%$ motif-similarity as members of the same group (Ross $e t$ al., 2001). In detail, UGT76E1 and UGT76E2 as well as UGT76E11 and UGT76E12 respectively cluster as homologs (Figure 4-2). The group $\mathrm{H}$ of the glycosyltransferase family has not been investigated in detail yet. No studies have been done on UGT76E1, UGT76E2 in particular, while UGT76E11 and UGT76E12 have been described to glycosylate flavonoids like quercetin (Li et al., 2018; Lim et al., 2004).

As mentioned, all UGTs in A. thaliana share a common structure with two protein domains (see 0 ) each harboring a conserved catalytic motif for substrate binding (N-terminus) and UDPbinding (C-terminus, Figure 1-5) (Offen et al., 2006). Therefore, UGT76E1, UGT76E2, UGT76E11, and UGT76E12 were analyzed for these catalytic motifs.

The two UGT-specific motifs of UGT76E1, UGT76E2, UGT76E11, UGT76E12, the control UGT74F1, and the UGT reference gene of $V$. vinifera VvGT1 (Figure 1-5) (Offen et al., 2006) 
were aligned with the Geneious algorithm. All sequences show the catalytic histidine at the Nterminus at the position 27 of the consensus sequences (Figure 4-3A). In the N-terminal regions, 14 of 57 amino acids of the candidates are similar to the reference VvGT1 (> $60 \%$ similarity, Figure 4-3A). The C-termini of the UGTs show high similarity, too. All six sequences share the UDP-Glc binding motif (Figure 4-3B, see Figure 1-5A). The candidate-UGTs show similarity for 39 of 57 amino acids in comparison to the reference VvGT1 (> $60 \%$ similarity, Figure 4-3B).

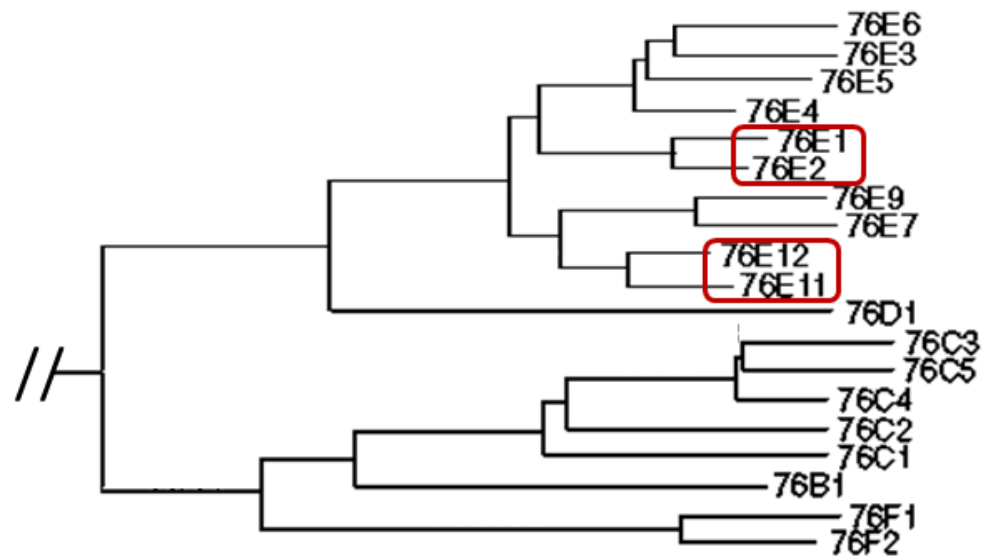

Figure 4-2: Phylogenetic tree of group H UGTs in A. thaliana

Phylogenetic tree of 19 out of 107 UGT genes of A. thaliana. The selected branch shows the group H (> $60 \%$ motif-similarity). The four UGTs, which are potentially active in the JA-pathway are marked with red boxes. The complete phylogenetic tree is given in Supplemental figure 1. Figure was taken from (Ross et al., 2001).

A)

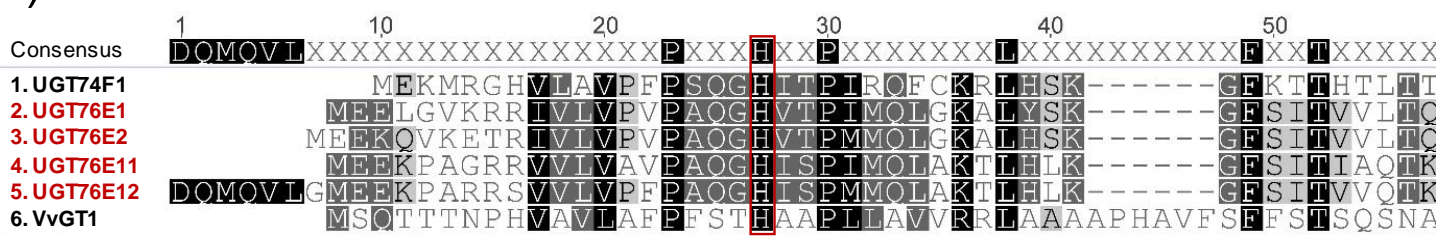

B)

Consensus
1. UGT74F1
2. UGT76E1
3. UGT76E2
4. UGT76E11
5. UGT76E12
6. VvGT1

Figure 4-3: UGT76E1, UGT76E2, UGT76E11, and UGT76E12 show the catalytic motifs

Amino acid alignment of the four UGT76Es (2. - 5.), the control UGT74F1 (1.), and the reference VvGT1 (6.). Amino acids, which are conserved in the six indicated UGTs, are highlighted. Amino acids highlighted by black boxes share $100 \%$ identity. Amino acids highlighted by dark grey, light grey or white boxes show 80 to $100 \%, 60$ to $80 \%$, or less than $60 \%$ similarity, respectively. A) Amino acid alignment and consensus sequence for the $\mathrm{N}$-termini are shown. The catalytic histidine $(\mathrm{H})$ at position 27 is marked by a red box. It was postulated by the x-ray crystal structure of VvGT1 and UGT74F1 (George Thompson et al., 2017; Offen et al., 2006). B) Amino acid alignment and consensus sequence of the Ctermini. The region is proposed to harbor the UDP-Glc binding motif (shown in red boxes) - postulated by the x-ray crystal structure of VvGT1 with the bound cofactor (Offen et al., 2006). Alignments were created with Geneious algorithm as global alignment with free end gaps and Blosum62 cost matrix in Geneious version 8.1 (Biomatters, New Zealand). Available from http://www.geneious.com. 
Because the four candidates are highly conserved in the substrate and UDP-Glc binding-motifs, the complete amino acid sequences were compared among each other. UGT76E1 and UGT76E2 show a high amino acid sequence identity of $88 \%$. Compared to UGT76E11, both, UGT76E1 and UGT76E2, display a sequence identity of $65 \%$. With UGT76E12, UGT76E1 and UGT76E2 share 65 and $67 \%$ identity respectively. UGT76E11 and UGT76E12 have $84 \%$ of the amino acids in common (Table 4-3). Taken together, all four UGTs are similar on the amino acid level with at least $65 \%$ identity. This confirms the systematic classification to the subfamily E $(\geq 60 \%$ amino acid sequence homology) (Mackenzie et al., 1997).

Table 4-3: Amino acid sequence comparison for UGT76E1, UGT76E2, UGT76E11, and UGT76E12

Amino acid identity of the indicated UGTs was calculate and the respective identity is given in \%. Alignments were created with the Geneious algorithm as global alignment with free end gaps and Blosum62 cost matrix in Geneious version 8.1 (Biomatters, New Zealand). Available from http://www.geneious.com.

\begin{tabular}{c|cccc}
\hline Amino acid identity & UGT76E1 & UGT76E2 & UGT76E11 & UGT76E12 \\
\hline UGT76E1 & & 88 & 65 & 67 \\
UGT76E2 & 88 & & 65 & 65 \\
UGT76E11 & 65 & 65 & & 84 \\
UGT76E12 & 67 & 65 & 84 & \\
\hline
\end{tabular}

\subsubsection{PROTEIN PARAMETERS OF UGT76E1, UGT76E2, UGT76E11, AND UGT76E12 ARE SIMILAR}

To get a better understanding of the four UGTs for the following protein purification and characterization, the protein parameters were estimated using the ProtParam online tool (https://web.expasy.org/protparam/). The MWs of the UGTs are about $51 \mathrm{kDa}$. The specific extinction coefficients at $280 \mathrm{~nm}$ are between $55.5 \mathrm{kM}^{-1} \mathrm{~cm}^{-1}$ (UGT76E12) and $66.4 \mathrm{kM}^{-1} \mathrm{~cm}^{-1}$ (UGT76E1). The predicted extinction coefficients were used to determine the exact concentrations of the purified proteins by spectroscopy later on. The predicted pIs of the proteins are acidic and mainly similar for UGT76E2 (pH 5.4), UGT76E11 (pH 5.9), UGT76E12 (pH 6.0), and UGT74F1 (pH 5.5). Interestingly, UGT76E1 has a $\mathrm{pI}$ at $\mathrm{pH}$ 6.6, which differs most to its homologue UGT76E2 (pI 5.4). Sequence analyses did not identify any signal peptides or transmembrane domains in any of the sequences. In addition, target sequences are not predicted for UGT76E2, UGT76E11, and UGT76E12. Again, UGT76E1 was predicted differently with a putative mitochondrial location. The score was the lowest prediction probability that the TargetP tool gives (Supplemental table 1).

The protein parameters illustrate that the five different UGTs are very similar in respect to their calculated properties. Only, UGT76E1 may show a less acidic pI and an uncertain putative 
mitochondrial location (Supplemental table 1). In respect to the characterization of the four candidates, the predictions do not give reasons to remove any motifs from the sequences.

\subsection{BIOCHEMICAL CHARACTERIZATION OF UGT76E1, UGT76E2, UGT76E11, AND UGT76E12}

After the four candidate genes have been identified by co-expression analyses and first in silico investigations have been done, the coding sequences were cloned, the proteins were heterologously expressed in E. coli, purified and introduced to biochemical characterizations. Likewise, it was aimed to determine their preferred substrates with various types of activity assays. The four UGTs were tested towards potential substrates, which are typically containing free hydroxy and carboxy groups including the proposed native substrate 12-OH-JA. Additionally, the enzymatic performance of the homogeneous UGTs were analyzed in a nativelike environment. Finally, the kinetic parameters for the main substrates were determined in a continuous spectrophotometric assay.

\subsubsection{UGT76E1, UGT76E2, UGT76E11, UGT76E12, AND UGT74F1 WERE HETEROLOGOUSLY EXPRESSED IN E. COLI}

The coding sequences of UGT76E1, UGT7TE2, UGT76E11, UGT76E12, and UGT74F1 were amplified from cDNA. These RNA-based templates ensure correct splicing and processing of the gene products, which should result in functional proteins after heterologous expression in E. coli. The templates for UGT76E1, UGT7TE2, UGT76E11, UGT76E12 were synthesized from RNA of leaf material $2 \mathrm{hpw}$. The coding sequence of $U G T 74 F 1$ was amplified as splice variant 2 (see 3.2.3.1) from cDNA, which are synthesized from RNA of unwounded root material. The coding sequences of all five genes have been integrated into the open reading frame (ORF) of the pET28a vector (Supplemental figure 10). The vector is optimized for protein expression and harbors an $\mathrm{N}$-terminal HisTag for affinity chromatography purification.

To express the five UGTs in E. coli with high efficiency as soluble proteins, different optimizations have been done to the expression system. Best results were obtained for expressing the proteins with the pET28a vector in BL21 Star (DE3) cells in the auto-induction medium ZYP5052 (Table 3-8) (Studier, 2005).

After protein expression, the heterologously expressed proteins can be visualized by SDS-PAGE in lysates of whole bacteria cells (Figure 4-4). There, signals of the expressed UGTs ( 51 kDa) can be seen, which are absent in the empty vector control. However, UGT74F1 is the only expressed protein, which illustrates strong signals at the predicted size of the UGT protein. UGT76E1, UGT76E2, UGT76E11, and UGT76E12 display faint signals at the assumed 
molecular weight of the expressed proteins. To detect the expressed proteins specifically in the whole cell lysates, the heterologous proteins were detected by immuno-blotting of their HisTags. The western blot shows a prominent signal for UGT76E2 and UGT74F1 at $51 \mathrm{kDa}$. In addition, a faint signal is visible for UGT76E11. No staining is visible for UGT76E1 and UGT76E12 (Figure 4-4B), however signals of the SDS-PAGE suggest for protein expression of UGT76E1 and UGT76E12.

A)

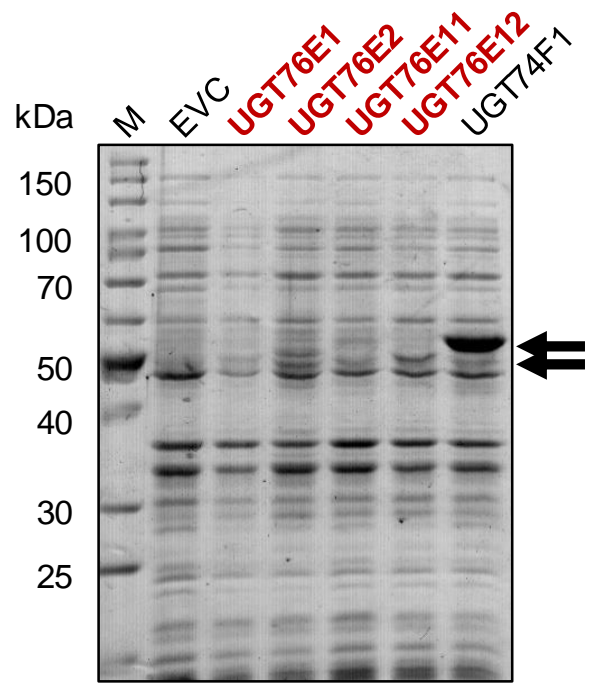

B)

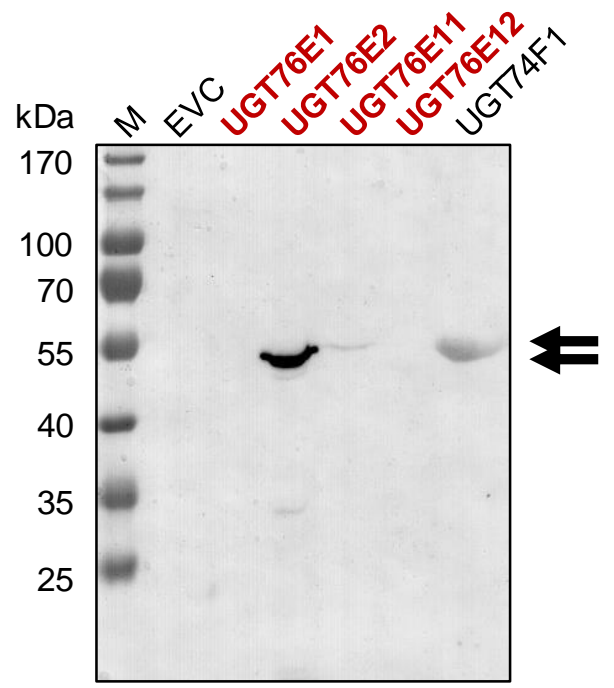

Figure 4-4: UGT76E1, UGT76E2, UGT76E11, UGT7 E12, and UGT74F1 were expressed in E. coli Indicated UGTs were fused to an N-terminal His-tags of pET28a vectors and heterologously expressed in E. coli BL21 Star (DE3) cells in auto-induction medium for $3 \mathrm{~d}$ at $16^{\circ} \mathrm{C}$. The heterologous protein expression was recorded in $100 \mathrm{~mL}$ E. coli cultures: A) Sodium dodecyl sulfate (SDS)-polyacrylamide gel electrophoresis (PAGE) of cultures expressing UGT76E1, UGT76E2, UGT76E11, UGT76E12, UGT74F1, and the empty vector control (EVC). After protein expression, $5 \mu \mathrm{L}$ of the samples were loaded onto a $10 \%$ SDS-PAGE for separation. The proteins were stained with $0.01 \%$ Coomassie Brilliant Blue. The arrows indicate the size of UGT proteins at appr. $50 \mathrm{kDa}$. The expressions and the SDS-PAGE are representative for at least five independent expressions for every enzyme. B) Western blot of the UGTs. The his-tagged UGTs were specifically detected via primary and secondary antibody and an activity staining by phosphatase. The arrows indicate the size of UGT proteins of about $51 \mathrm{kDa}$. The blot was performed like this once.

\subsubsection{UGT76E1, UGT76E2, UGT76E11, UGT76E12, AND UGT74F1 WERE PURIFIED TO HOMOGENEITY}

The biochemical investigation of so far not characterized enzymes requires homogeneous proteins. Therefore, a two-step protein purification was set up to yield homogeneous UGT76E1, UGT76E2, UGT76E11, UGT76E12 and UGT74F1. Using different separation properties, two purification steps ensure that co-purifications of unspecific proteins are diminished. First, Histagged proteins are captured from the bacterial lysate by IMAC. Second, the UGTs are further purified by SEC. 
Briefly, the cleared lysate was loaded onto the IMAC-column to retard His-tagged proteins from other bacterial proteins. Since those unspecific proteins were not (or rarely) bound to the bivalent cations of the column material, they could be washed away by loading buffer His A. The remaining captured proteins were eluted by imidazole.

Even though UGT76E1, UGT76E2, UGT76E11 and UGT76E12 show very high amino acid sequence homologies (seen Table 4-3), it was not possible to use one common scheme for efficient and reliable purification of all UGTs up to homogeneity. The protein purification of the UGT enzymes required several optimizations for each enzyme.

In this regard, the purification of UGT76E1 was most challenging and different parameters have been improved to produce a sufficient yield of active protein. Firstly, the cell pellet of $0.5 \mathrm{~L}$ was resolved in $20 \mathrm{mM}$ Tris/ $\mathrm{HCl} \mathrm{pH}$ 7.5, $50 \mathrm{mM} \mathrm{NaCl}$. Protein elution from the IMAC-material with $150 \mathrm{mM}$ imidazole resulted in a small UGT76E1 protein fraction (Figure 4-5A). However, the enriched UGT76E1 protein precipitated immediately and did not show any glycosyltransferase activity (data not shown). To improve the IMAC-purification step, the culture volume was enlarged stepwise to $2 \mathrm{~L}$ and the bivalent cation was exchanged from nickel to cobalt. Cobalt as chelating metal cation forms less strong chelate complexes with the His-tagged proteins and, thus, releases the proteins at lower imidazole concentrations. The reduction to $40 \mathrm{mM}$ imidazole increased the protein stability. Additionally, the concentration of the Tris-buffer, its $\mathrm{pH}$-value and the salt content were optimized to $50 \mathrm{mM}$ Tris/ $\mathrm{HCl} \mathrm{pH} 9.0$, and $100 \mathrm{mM} \mathrm{NaCl}$. In addition, $0.1 \%$ Tween 20 was added to improve solubility of UGT76E1. Finally, these changes of the IMACpurification resulted in a sufficient yield of active and stable UGT76E1 (Figure 4-5B-D).

Similar optimizations have been done for UGT76E2, UGT76E11, UGT76E12, and the control protein UGT74F1. The cell pellet of $2 \mathrm{~L}$ culture (1 L for UGT74F1) was used for optimized settings. Improving purification procedures resulted in specific buffer requirements for each enzyme (Table 3-16 - Table 3-20) with varying concentrations of Tris/HCl-buffer (20 - $50 \mathrm{mM}$ ), $\mathrm{NaCl}(50-100 \mathrm{mM})$, different $\mathrm{pH}$ values $(7.5-9.0)$, and in part requirements for $0.1 \%$ Tween 20 or $10 \%$ glycerol. To increase the power of the IMAC purification and to remove impurities, a special wash buffer (50 mM Tris/HCl pH 7.5, $100 \mathrm{mM} \mathrm{NaCl,} 2 \mathrm{mM}$ DTT, $5 \mathrm{mM}$ ATP, $5 \mathrm{mM}$ $\mathrm{MgCl}_{2}, 20 \mathrm{mM}$ imidazole, Table 3-21) was developed for purification of UGT76E2, UGT76E11, and UGT74F1. Here, ATP provides molecular energy to remove chaperons from the columnresin or the proteins of interest. Following, the retained proteins were eluted from the column material by specific imidazole concentrations (appr. $150 \mathrm{mM}$ for nickel- and appr. $40 \mathrm{mM}$ for cobalt-affinity column resin). As second purification step, SEC was used.

After optimization, the IMAC protein purifications of five UGTs show similar behaviors. After cell lysis, the majority of UGT76E1, UGT76E2, and UGT76E11 was soluble in the lysate fraction 

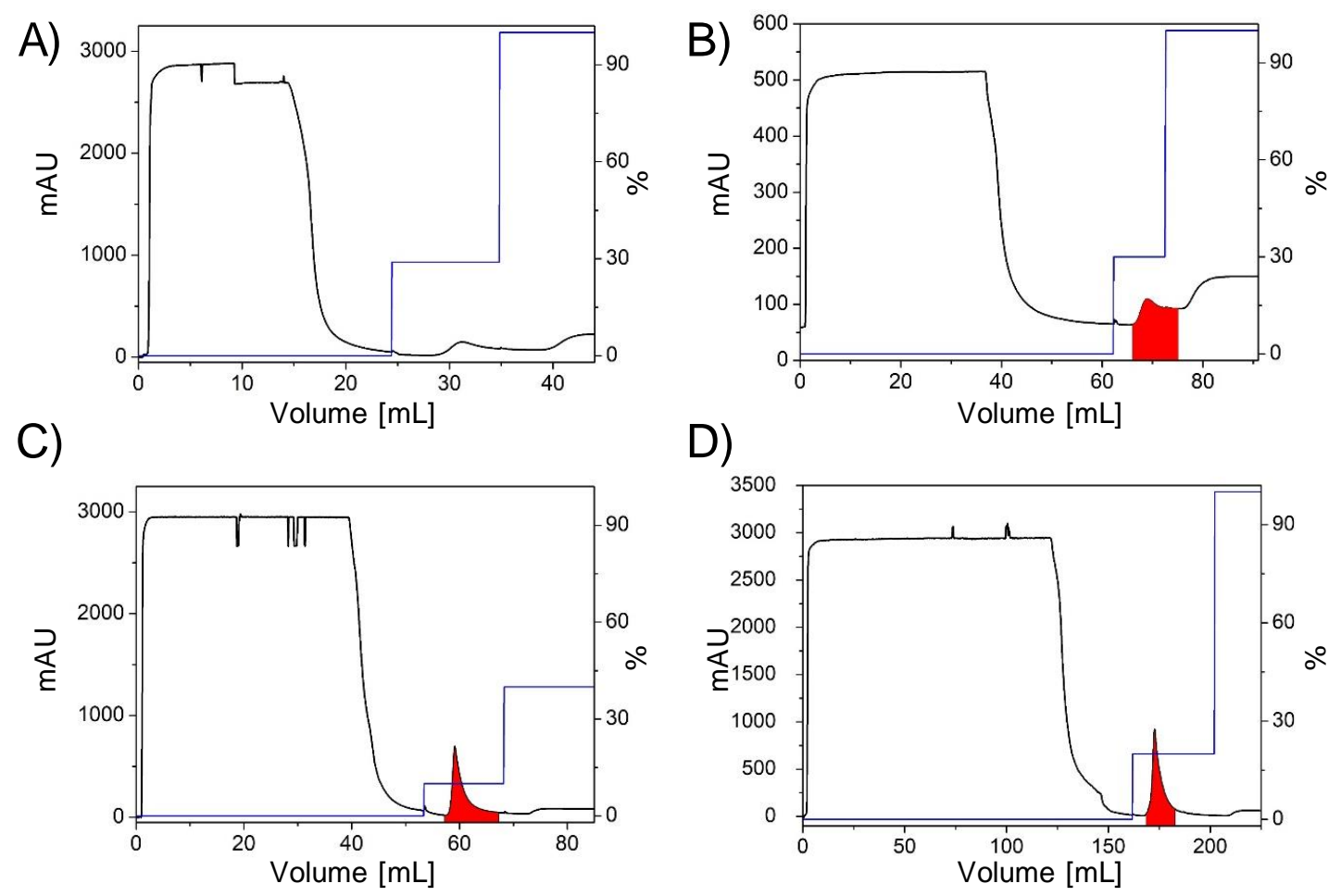

Figure 4-5: Optimization of the protein purification of UGT76E1 by affinity chromatography

UGT76E1 was fused to an N-terminal HisTag, heterologously expressed in E. coli BL21 Star (DE3) cells and purified by immobilized metal affinity chromatography (IMAC). The chromatograms illustrate the absorption at $280 \mathrm{~nm}$ (milli absorption units (mAU)) and the concentration of elution buffer (\%) over the elution volume. Elution of active UGT76E1 is depicted in red area. IMAC for UGT76E1 was optimized in the following way: A) $0.5 \mathrm{~L} \mathrm{E}$. coli lysate with nickel affinity, $20 \mathrm{mM} \mathrm{Tris} / \mathrm{HCl} \mathrm{pH} \mathrm{7.5,50} \mathrm{mM} \mathrm{NaCl}$.

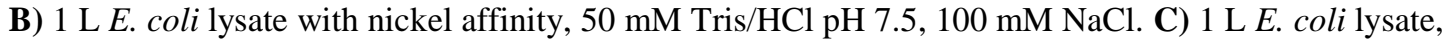
cobalt instead of nickel as binding metal for IMAC, $50 \mathrm{mM}$ Tris/ $\mathrm{HCl} \mathrm{pH} 7.5,100 \mathrm{mM} \mathrm{NaCl}, 0.1 \%$ Tween 20. D) $2 \mathrm{~L} E$. coli lysate with cobalt affinity, $50 \mathrm{mM}$ Tris/ $/ \mathrm{HCl} \mathrm{pH} 9.0,100 \mathrm{mM} \mathrm{NaCl}, 0.1 \%$ Tween 20. Each profile is representative for at least three independent purifications.

whereas large amounts of UGT76E12 and UGT74F1 remained in the pellet fraction. Following necessary (mainly for UGT76E2, UGT76E11, and UGT74F1) the special wash buffer improved this purification step. As shown in Figure 4-6, all chromatograms display specific protein elution at the applied imidazole concentrations. The fractions show enriched UGTs with some remaining impurities (SDS-PAGE, Figure 4-6). Only, UGT76E2 shows several additional protein signals. The UGT-enriched fractions of the IMAC were introduced to SEC. As shown in Figure 4-6 the chromatograms generally illustrate two protein peaks. For UGT76E1, both fractions were tested for activity (60 $\mu \mathrm{g}$ protein, $20 \mu \mathrm{M} \omega$-OH-16:0, $5 \mathrm{mM}$ UDP-Glc, $16{ }^{\circ} \mathrm{C}, 2 \mathrm{~h}, \mathrm{LC}-\mathrm{MS}$ analysis). The protein, which elutes in the first peak at $48 \mathrm{ml}$ did not show activity and represents heterooligomeric UGT76E1 whereas second peak at $63 \mathrm{~mL}$ showed UGT-activity and corresponds to the MW of monomeric UGT76E1 (data not shown, $c f$. Figure 4-7). Hence, the UGT-proteins, which elute later (second peak) were collected and used as purified UGTs for further analysis. The respective samples illustrate homogenous protein signals at the MWs of the five UGTs with some smaller proteins signals at lower MWs. Most likely, those indicate degradation because the signals increase over time (Figure 4-6). 


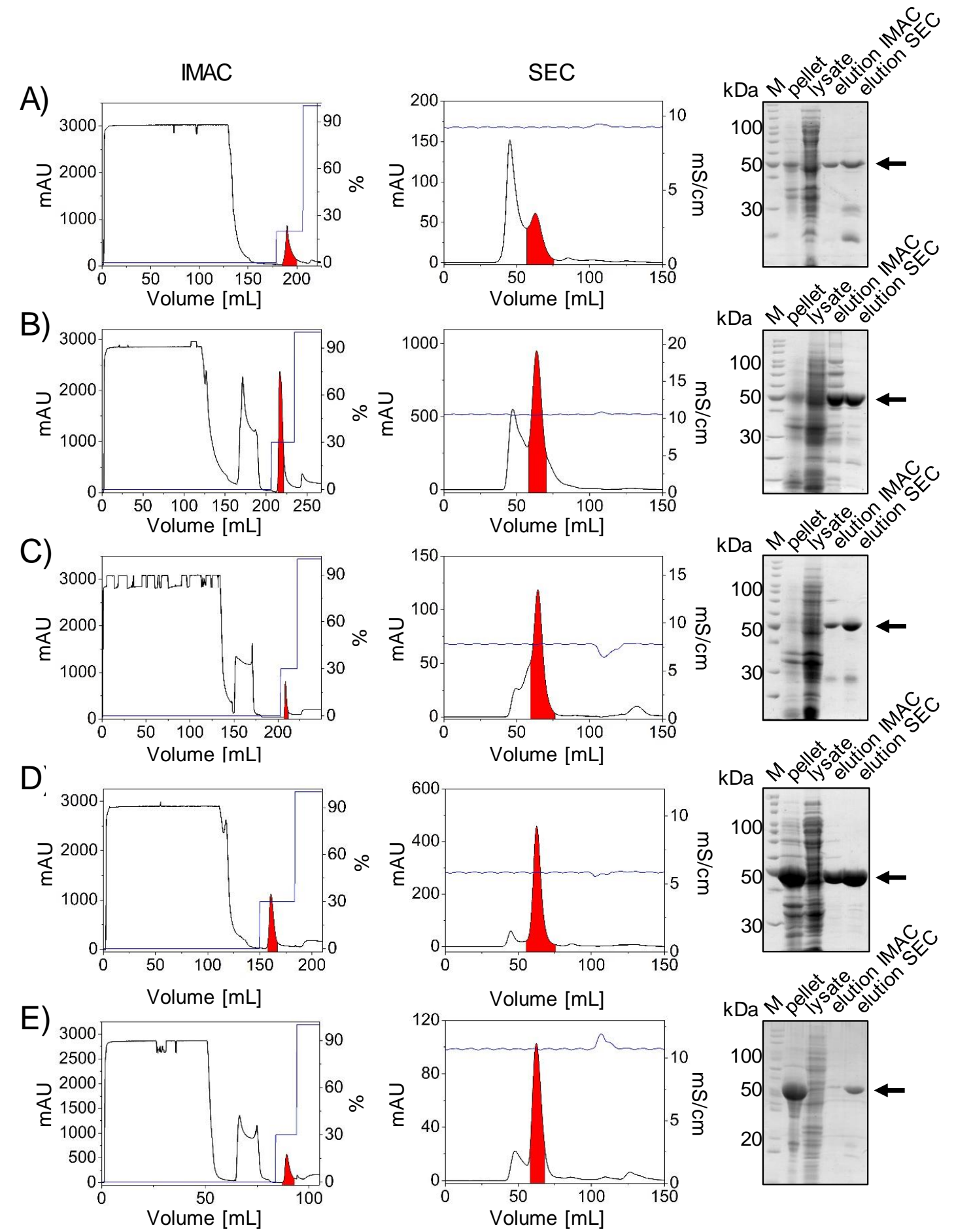

Figure 4-6: Protein purification for UGT76E1, UGT76E2, UGT7E11, UGT76E12, and UGT74F1

UGTs fused to an N-terminal His-tag and heterologously expressed in E. coli BL21 Star (DE3) were purified by a two-step protein purification strategy of immobilized metal affinity chromatography (IMAC) with nickel as bivalent cation and size exclusion chromatography (SEC). Shown are the purification chromatograms and a sodium dodecyl sulfate (SDS)-polyacrylamide gel electrophoresis (PAGE) with samples of pellet, lysate, IMAC eluate, and SEC eluate. Arrows indicate the size of the respective purified protein. Chromatograms illustrate the absorption at $280 \mathrm{~nm}$ (milli absorption units (mAU)) during elution. For IMAC, the second y-axis shows the concentration of elution buffer (\%). For SEC, the second y-axis shows the conductivity $(\mathrm{mS} / \mathrm{cm})$. The volume that contained the protein of interest is marked in red. Purifications of A) UGT76E1 B) UGT76E2 C) UGT76E11 D) UGT76E12 E) UGT74F1. The depicted purifications are representative for at least four independent purifications for each UGT. 
Taken together, the two-step purification strategy resulted in mainly homogeneous proteins of UGT76E1, UGT76E2, UGT76E11, UGT76E12, and UGT74F1. Lowest protein yields were obtained for UGT76E1 (appr. $0.5 \mathrm{mg} / \mathrm{L}$ culture) and highest yields were achieved for UGT76E2 $(5.0 \mathrm{mg} / \mathrm{L}$ culture $)$. In the end, the proteins were concentrated as stocks (>1 mg/mL) and stored at $-80{ }^{\circ} \mathrm{C}$.

\subsubsection{UGT76E1, UGT76E2, UGT76E11, UGT76E12, AND UGT74F1 SHOW ACTIVITY TOWARDS $\omega$-OH-HEXADECANOIC ACID AND SOME TOWARDS 12-OH-JA}

After heterologous expression and purification of the five UGTs, the uncharacterized UGTs were tested for their catalytic activity. Therefore, two tentative substrates were used: 12-OH-JA and $\omega$ $\mathrm{OH}-16: 0.12-\mathrm{OH}-\mathrm{JA}$ is the expected substrate for the four selected UGTs. The hydroxy-fatty acid $\omega-\mathrm{OH}-16: 0$ should be used as alternative substrate, which is commercially available in contrast to 12-OH-JA. As mentioned above, P450 enzymes (CYP94B1, CYP94B3, and CYP94C1), which catalyze the oxidation of JA-Ile (see 1.1.3) also tolerate fatty acids for catalysis (Benveniste $e t$ al., 2006). Moreover, some UGTs are capable to glycosylate hydroxy-fatty acids (Huang et al., 2015). Hence, UGT76E1, UGT76E2, UGT76E11, and UGT76E12 putatively active on 12-OH-JA might also tolerate FA-derivatives for their catalysis.

In the assay, $10 \mu \mathrm{g}$ homogeneous UGT76E1, UGT76E2, UGT76E21, UGT76E12, UGT74F1, and a heat-inactivated control (aliquots of all enzymes) were incubated with $0.1 \mathrm{mM} \mathrm{\omega -OH-16:0}$ or 12-OH-JA, respectively, and the reaction products were analyzed by LC-MS. Here, LC-MS analyses provide high sensitivity to detect even small amounts of enzymatic products at a specific retention time (RT). The exact $\mathrm{m} / \mathrm{z}$ of the expected products was used to extract the specific mass signal. The extracted ion chromatograms (EICs) for $\omega$-glucosyl- $O$-hexadecanoic acid $(\omega$ - $O$-Glc-

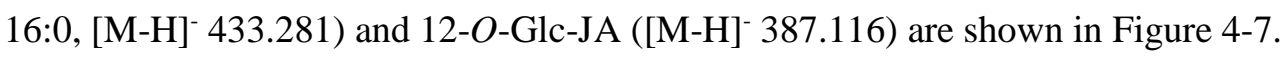

All tested enzymes used $\omega-\mathrm{OH}-16: 0$ as substrate, at least to a limited extent. The product $\omega-O$ Glc-16:0 elutes at $5.8 \mathrm{~min}$. It is formed in different amounts by UGT76E12 (100\%), UGT76E2 (70 \%), UGT74F1 (15\%), UGT76E1 (10\%), and only trace amounts by UGT76E11 (Figure 4-7A). Next, the UGTs were tested towards $12-\mathrm{OH}-\mathrm{JA}$. The product $12-\mathrm{O}$-Glc-JA elutes at $3.3 \mathrm{~min}$. Here, the highest activity is depicted by UGT76E2 (100\%), UGT76E1 and UGT76E12 shows $65 \%$, and UGT76E11 illustrates trace amounts of product. UGT74F1 shows no product signal (Figure 4-7B).

In summary, the activity assay uncovers enzymatic activity of all five UGTs towards the substrate $\omega-\mathrm{OH}-16: 0$ (for UGT76E11, at least to a minor extent). The reaction serves as positive control for enzymatic activity in the following. 12-OH-JA was used as substrate by UGT76E2, 
UGT76E1, and UGT76E12. These enzymes form 12-O-Glc-JA in vitro and, likewise, can be classified as 12-OH-JA UDP-glycosyltransferases.

A)
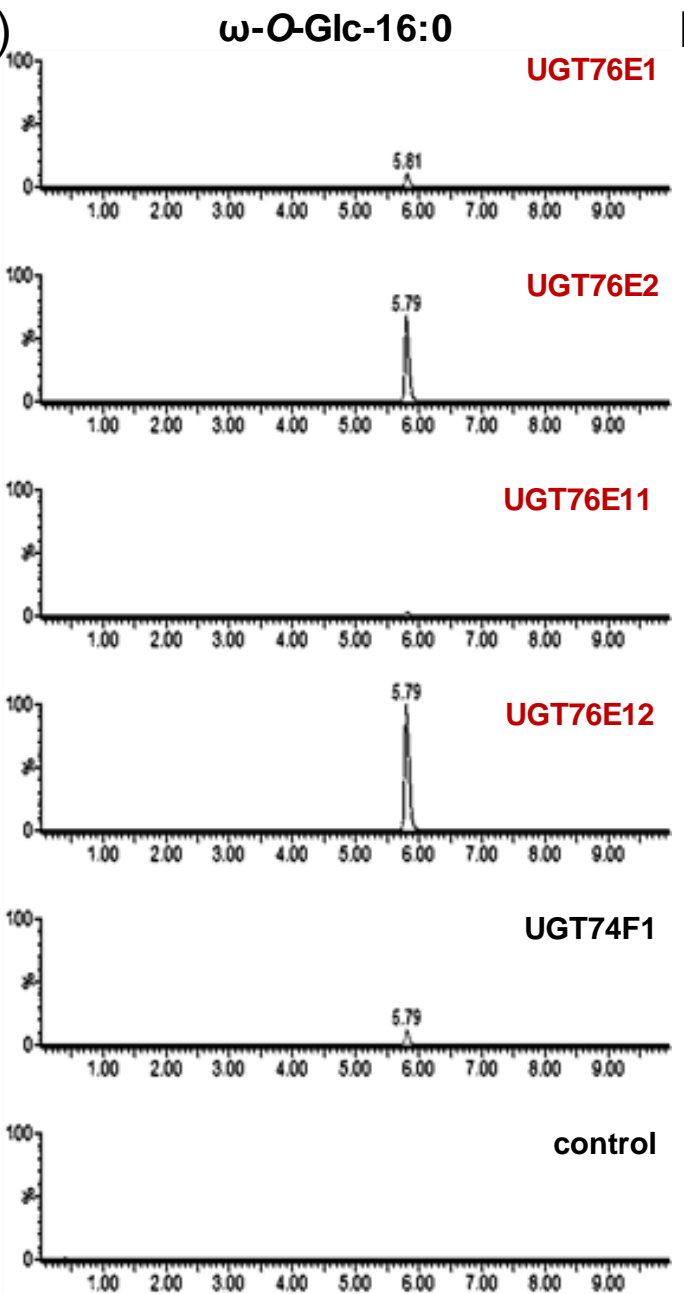

B)
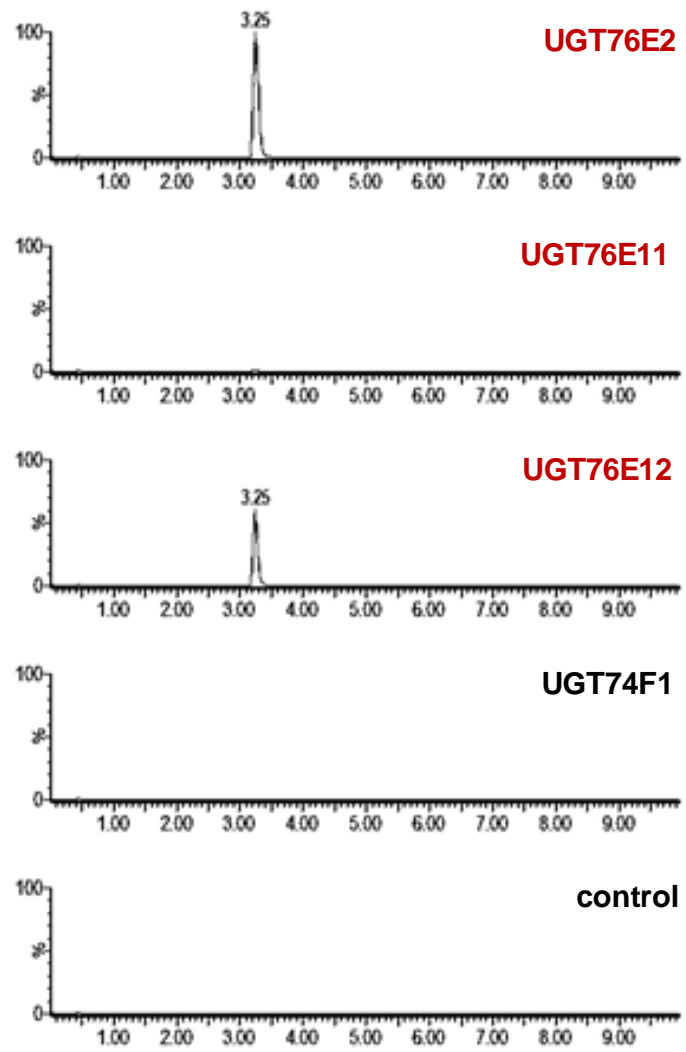

Figure 4-7: LC-MS based activity assays of UGT76E1, UGT76E2, UGT7E11, UGT76E12, and UGT74F1 with $\omega$-OH-hexadecanoic acid and 12-hydroxy-JA as substrates

Activity tests of homogeneous UGT76E1, UGT76E2, UGT76E21, UGT76E12, UGT74F1, and inactivated UGTs as negative control were performed with $0.1 \mathrm{mM} \omega$-hydroxy-hexadecanoic acid or 12hydroxy-JA and $0.5 \mathrm{mM}$ UDP-Glc for $1 \mathrm{~h}$ at $25^{\circ} \mathrm{C}$. The reactions were stopped by adding $25 \mu \mathrm{L}$ acetonitrile and analyzed by LC-MS. Extracted ion chromatograms (EICs) are shown for the products A) $\omega$-glucosyl- $O$-hexadecanoic acid ( $\omega$-O-Glc-16:0, [M-H] $]^{-} 433.281$ ) and B) 12-O-glucosyl-JA (12-O-Glc$\left.\mathrm{JA},[\mathrm{M}-\mathrm{H}]^{-} 387.116\right)$. Y-Axes are scaled to the highest product signal (100\%). Data represent a single experiment. Measurements were performed by Dr. Kirstin Feussner (University of Goettingen, Germany).

To obtain information about the side of glycosylation (at the hydroxyl or the carboxy group of 12-OH-JA), the product of the UGT76E2-reaction with 12-OH-JA (see above) was analyzed and compared to an authentic standard of 12-O-Glc-JA (kindly provided by Dr. Otto Miersch, University of Halle/Wittenberg, Germany) by MS/MS analyses. The LC-MS analysis of the enzymatic product shows two signals in the total ion chromatogram (TIC). The second signal could be assigned to the substrate 12-OH-JA. The first signal at 3 min shows the same RT as the 
standard (Figure 4-8A, C). Both signals share the $\mathrm{m} / \mathrm{z}$ of $[\mathrm{M}-\mathrm{H}]^{-} 387.116$ and are identical in respect to their fragmentation patterns. All major fragments could be assigned to $12-O$-Glc-JA including the analytical fragment of $m / z$ 207.101, which derives from the neutral loss of the Glcmoiety and $m / z, 59.013$, which is specific for the $\alpha$-carboxy group of jasmonates (Figure 4-8B, D, E). Therefore, 12-O-Glc-JA could be unequivocally confirmed as the product of the UGT76E2, UGT76E1 and UGT76E12 catalyzed reaction with 12-OH-JA.

A)

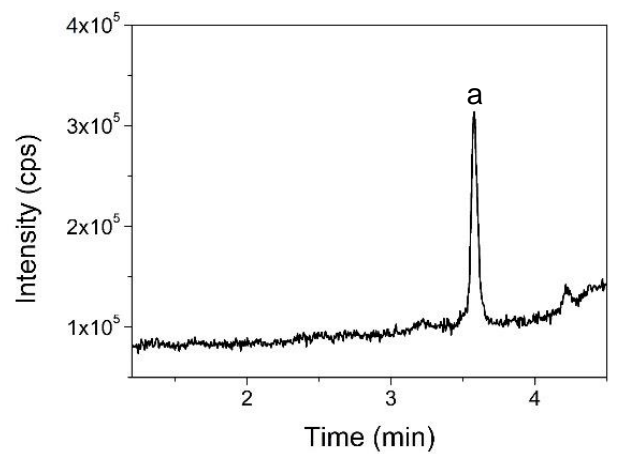

C)

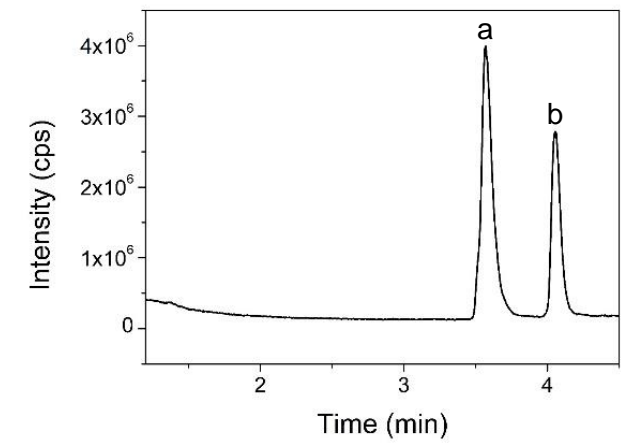

B)

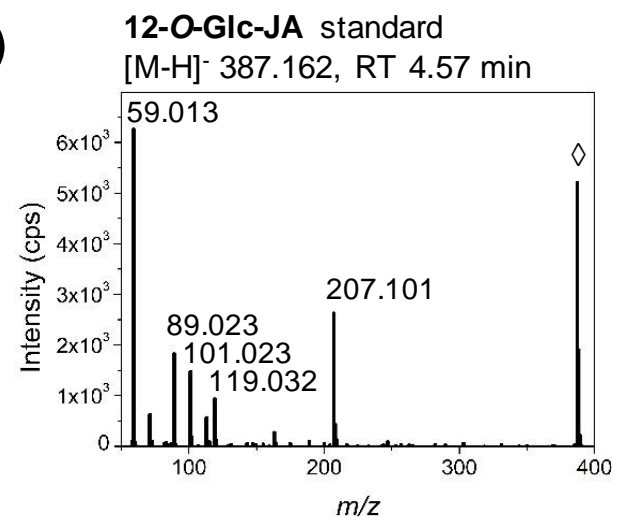

D)

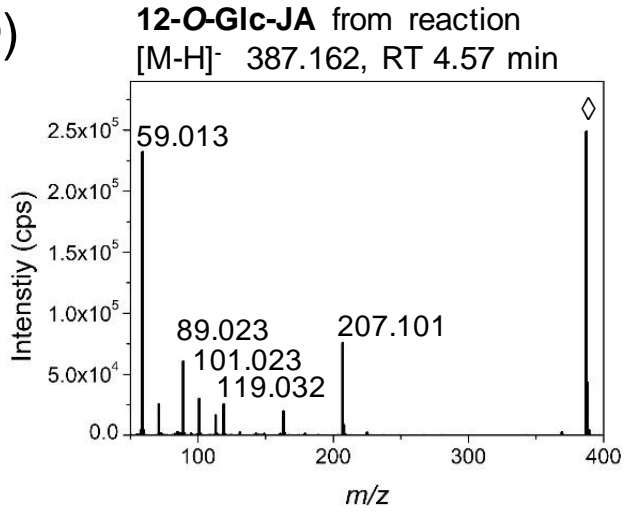

E)

\begin{tabular}{|c|c|c|c|c|}
\hline & & $m / z$ & $\begin{array}{c}\text { Elemental } \\
\text { composition }\end{array}$ & Origin \\
\hline & $\diamond$ & 387.162 & $\mathrm{C} 18 \mathrm{H} 2809$ & \\
\hline $111 \mathrm{IV}$ & I & 207.101 & $\mathrm{C} 12 \mathrm{H} 15 \mathrm{O} 3$ & JA \\
\hline II, III, IV & ॥ & 119.032 & $\mathrm{C} 4 \mathrm{H} 7 \mathrm{O} 4$ & Glc \\
\hline & III & 101.023 & $\mathrm{C} 4 \mathrm{H} 5 \mathrm{O} 3$ & Glc \\
\hline & IV & 89.023 & С3H5O3 & Glc \\
\hline $\mathrm{O}=\mathrm{OH}$ & V & 59.013 & $\mathrm{C} 2 \mathrm{H} 3 \mathrm{O} 2$ & JA \\
\hline
\end{tabular}

Figure 4-8: Structure confirmation of 12-O-glucosyl-JA

An authentic chemical standard of 12-O-glucosyl-JA was compared to the reaction product of UGT76E2 with 12-hydroxy-JA (12-OH-JA) by liquid chromatography coupled to tandem mass spectrometry (LCMS/MS). Shown are total ion chromatograms (TICs) and the MS/MS spectra in the negative ionization mode with collision energies of $20 \mathrm{eV}$. A) The TIC of 12-O-Glc-JA standard (a) and B) the corresponding MS/MS spectrum. C) TIC of the in vitro reaction of UGT76E2 with $0.1 \mathrm{mM} 12-\mathrm{OH}-\mathrm{JA}$ and $0.5 \mathrm{mM}$ UDPGlc (in $50 \mathrm{mM}$ Tris/ $\mathrm{HCl} \mathrm{pH} \mathrm{8.0,100} \mathrm{mM} \mathrm{NaCl}, 1 \mathrm{~h}$ at $25^{\circ} \mathrm{C}$ ). The chromatogram shows the signals for the product 12-O-Glc-JA (a) and the substrate 12-OH-JA (b). D) MS/MS spectrum of the respective 12-O-GlcJA of the enzymatic reaction. E) Assignment of the MS/MS-fragments to the structure of 12-O-Glc-JA. LCMS/MS analysis was performed by Dr. Kirstin Feussner. 


\subsubsection{THE UGT ENZYMES PREFER MILD ALKALINE PH}

With a common substrate in hands, UGT76E1, UGT76E2, UGT76E11, and UGT76E12 can be compared with respect to their optimal parameters. Since, UGTs are generally similar and tend to have rather broad specificities, it is important to characterize them in detail to determine their preferences. The optimal $\mathrm{pH}$ and temperature were determined for UGT76E1, UGT76E2, UGT76E11, and UGT76E12 with $\omega-\mathrm{OH}-16: 0$ as substrate. The formation of $\omega$-O-Glc-16:0 was detected by LC-MS as before. The product intensities were evaluated as relative signal intensities of the signal areas and the highest value was set to $100 \%$.

The optimal $\mathrm{pH}$ values were analyzed in the range of $\mathrm{pH} 3$ to $\mathrm{pH} 11$ in BRB (Britton \& Robinson, 1931). UGT76E1 shows two maxima at $\mathrm{pH} 7$ and $\mathrm{pH} 9$ (Figure 4-9A). UGT76E2 displays one optimum at pH 7.5 (Figure 4-9B). UGT76E11 and UGT76E12 show their optimum at pH 8. (Figure 4-9C, D). The pH optima of all four UGTs are rather similar with mild alkaline values of pH $7.0-8.0$.

A)

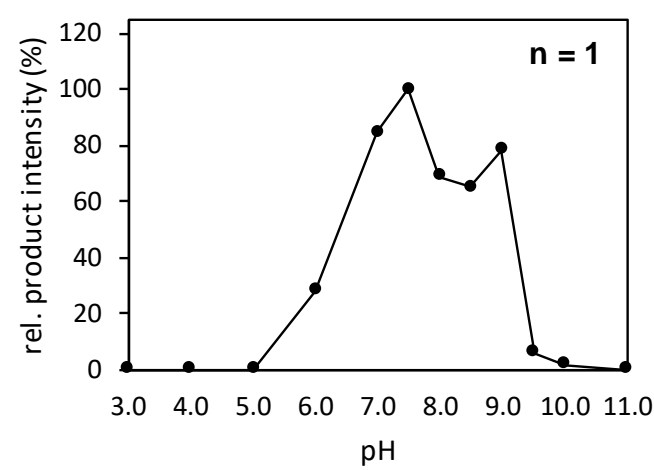

C)

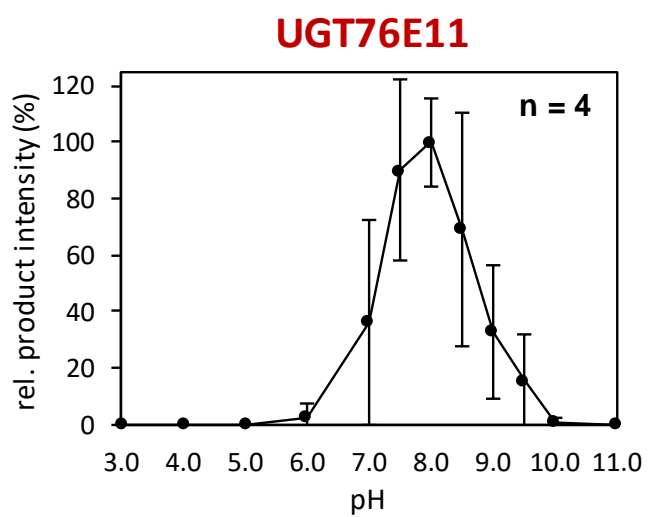

B)

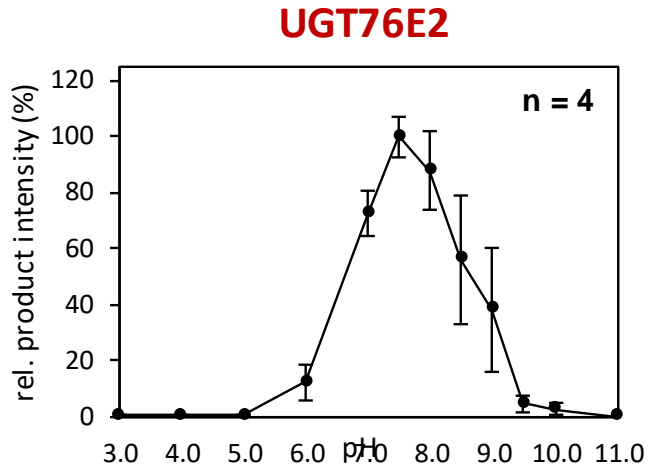

D)

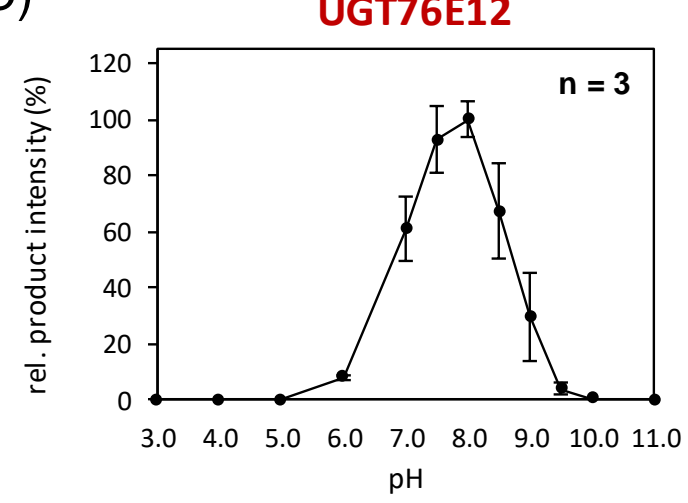

Figure 4-9: Optimum pH of UGT7E1, UGT76E2, UGT76E11, and UGT7E12

$\mathrm{pH}$ optima for the indicated UGTs were determined in the range of $\mathrm{pH} 3$ to $\mathrm{pH} 11$ in the Britton-Robinson buffer system. $0.1 \mathrm{mM} \omega$-hydroxy-hexadecanoic acid and $0.5 \mathrm{mM}$ UDP-Glc were incubated with enzyme at $25{ }^{\circ} \mathrm{C}$ for $1 \mathrm{~h}$. The product formation was detected by LC-MS and shown as relative signal intensities (\%). A) UGT76E1. B) UGT76E2. C) UGT76E11. D) UGT76E12. Data represents one experiment for UGT76E1. All other measurements are means with \pm SD of at least three independent experiments. 
The optimal temperatures of the UGTs were determined from $4{ }^{\circ} \mathrm{C}$ to $50{ }^{\circ} \mathrm{C}$. UGT76E1 shows enzymatic activity (>20\%) between $4-35{ }^{\circ} \mathrm{C}$ with an optimum at $20^{\circ} \mathrm{C}$. UGT76E2 shows activity between $15-42{ }^{\circ} \mathrm{C}$ and an optimum at $30^{\circ} \mathrm{C}$. UGT76E11 and UGT76E12 show enzymatic activity between $10-42{ }^{\circ} \mathrm{C}$ but with different optima - UGT76E11 at $25{ }^{\circ} \mathrm{C}$ and UGT76E12 at $30{ }^{\circ} \mathrm{C}$ (Supplemental figure 11).

\subsubsection{UGT76E1 AND UGT76E2 SHOW SUBSTRATE PREFERENCE FOR 12-OH-JA}

UGT enzymes tend to have rather broad substrate specificities (Bowles et al., 2006). Hence, it is necessary to test the specificities of the four UGTs with different substrates and compare their reaction performance. Therefore, a radioactive approach was established using ${ }^{14} \mathrm{C}$-labeled cosubstrate. The radioactive carbons are incorporated into the Glc moiety of the UDP-Glc. The UGTs transfer the ${ }^{14} \mathrm{C}(\mathrm{U})-\mathrm{Glc}$ moiety of the UDP-Glc onto the substrate. Like this, every reaction turnover produces the same signal intensity independently of the used substrate and the signal intensities of generated products can be compared to each other.

The substrate specificities of the UGT76E1, UGT76E2, UGT76E11, UGT76E12, and UGT74F1 were analyzed towards a set of different tentative substrates with different functional groups. Some of which harbored a carboxy group, some a hydroxy group, and other both. Hereby, the specificity of the UGTs towards forming GE- or Glc-products can be investigated. One sub-set included fatty acid derived compounds with a chain length of 16 carbons and without or with a hydroxy group at different positions, like hexadecanoic acid (16:0), $\omega-\mathrm{OH}-16: 0$, 2-hydroxy-16:0 (2-OH-16:0), 3-hydroxy-16:0 (3-OH-16:0). Additionally, a fatty alcohol with a chain length of 16 carbons was used (hexadecanol (OH-C16)). This first set has developed from the activity towards $\omega-\mathrm{OH}-16: 0$ and evaluates if the carboxy, the hydroxy group, both, or the position of the hydroxy group are essential for catalysis. A second group contained plant hormones and signal compounds as SA, pipecolic acid (Pip), ICA, ABA, zeatin, and GA. In addition, BA was included to discriminate against the hydroxy group of SA. This second set arose from the different activities of characterized UGTs towards small aromatic molecules (Lim et al., 2002) and will question a physiological specificity of the UGTs towards some phytohormones. However, the focus was given to a third set of JA-precursors and jasmonates including 12-OPDA, JA, 12-OH-JA, 11hydroxy-JA (11-OH-JA), 12-hydroxy-JA-methyl ester (12-OH-JA-ME). Here again, structural elements like a free or shielded carboxy group, the presence and position of a hydroxy group and the length of the C-terminus were investigated. Lastly, a fourth set of flavonoids contained quercetin, dihydro-myricetin (dh-myricetin), and dihydro-kaempferol (dh-kaempferol). These substrates can be glycosylated at different positions and have been used for broad activity tests before (Lim et al., 2004). A control was performed with heat-inactivated enzyme. For analysis, the reaction products were extracted with n-butanol (cf. Huang et al., 2015; Suzuki et al., 2007) and separated by TLC ( $c f$. Augustin et al., 2012). Here, all samples of one UGT were run together 
and treated equally. Hence, all product signals of one enzyme towards all substrates can be compared to each other qualitatively.

Noticeable, this assay pointed out side activities of UGT76E12 towards the alcohol-solvent ethanol (Supplemental figure 12). Thus, all substrates were dissolved in ACN. Finally, 20 aglycones with different structural properties were tested with the UGTs (Supplemental table 2).

UGT76E1 illustrates low signal intensities but a clear preference for 12-OH-JA (100\%). Minor activities were detected towards $\omega-\mathrm{OH}-16: 0$ (38\%), and zeatin (23\%). All three substrates share aliphatic chains with terminal hydroxy groups. In addition, 12-OH-JA and $\omega-\mathrm{OH}-16: 0$ contain a carboxy group whereas zeatin includes a purine (see Supplemental table 2). UGT76E2 shows similar preferences with higher intensities. The highest product signal is seen for 12-OH-JA $(100 \%)$. This enzyme also tolerated 11-OH-JA and 12-OH-JA-ME (both $13 \%$ ) as well as $\omega-\mathrm{OH}-$ 16:0, 3-OH-16:0, and zeatin (all appr. $7 \%$ ). Here, the exact $\omega$-terminal position of the hydroxy group seems to be less important and UGT76E2 tolerates the methylated carboxyl group of 12 OH-JA-ME as well (see Supplemental table 2). In the case of UGT76E11, the signal intensities are too low to judge them as enzymatic activities. On top of this, no clear specificity or preference can be derived from those signals. For UGT76E12, the highest activities are displayed for $\omega-\mathrm{OH}-$ 16:0 (100 \%), dh-kaempferol (63\%), 12-OH-JA (38 \%), and quercetin (25\%). Here, UGT76E12 showed dual specificity for the terminal hydroxy group of $\omega-\mathrm{OH}-16: 0$ and 12-OH-JA as well as for polyphenolic compounds like dh-kaempferol and quercetin (see Supplemental table 2). The control, UGT74F1, shows high product formation with its native substrate SA (100\%). Additional products were formed with BA, $\omega-\mathrm{OH}-16: 0$, ICA, 12-OPDA, 12-OH-JA, and 11-OHJA (all $6 \%$, Figure 4-10). Interestingly, UGT7674F1 displays side-activity towards substrates featuring a carboxy group, but no hydroxyl group (BA, ICA, 12-OPDA, JA, see Supplemental table 2).

Altogether, UGT76E1 and UGT76E2 specifically glycosylate 12-OH-JA with a high specificity and they do not use JA or 12-OH-JA-ME. UGT76E12 displays the highest activity towards $\omega$ OH-16:0 but neither the respective FA nor the fatty alcohol. All three, UGT76E1, UGT76E2 and UGT76E12 prefer substrates featuring both, a terminal hydroxy group and a carboxy function. Thereby, the hydroxy group is necessary but not sufficient for catalysis. Although this experiment cannot distinguish between a glycose ester and a glycoside being formed, the structure analysis has confirmed the glycoside of $12-O$-Glc-JA (see Figure 4-8). Hence, it is likely that glyosidic products are also formed in this experiment and the additional carboxy group assists catalysis. For UGT76E11 however, the evaluation of the activity and specificity was not possible in this experiment. 


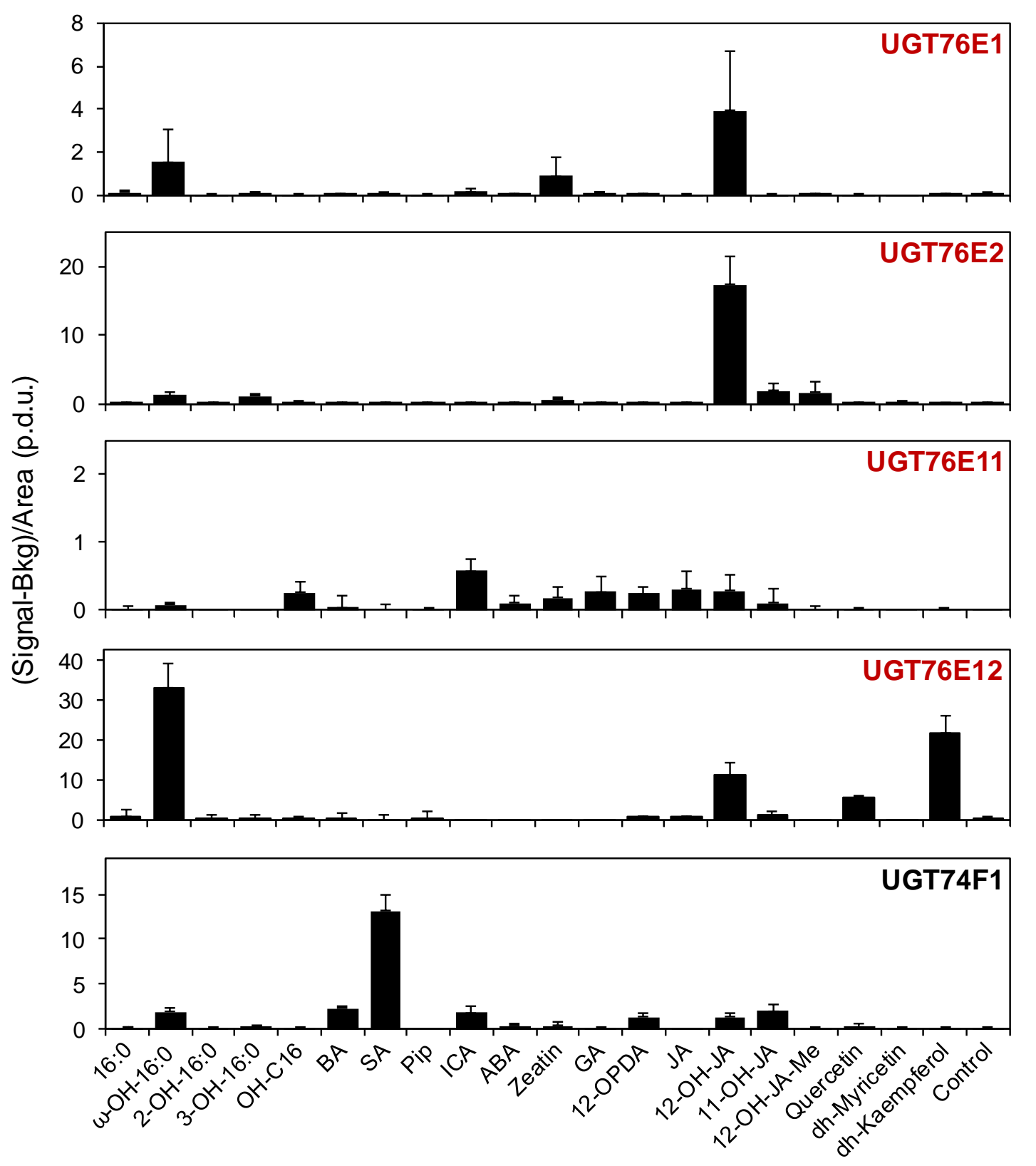

Figure 4-10: Substrate specificity of UGT7E1, UGT7E2, UGT76E11, UGT76E12, and UGT74F1

The substrate specificities of the UGTs were analyzed by an activity assay with ${ }^{14} \mathrm{C}$-labeled UDP-Glc (UDP- $\left.{ }^{14} \mathrm{C}(\mathrm{U})-\mathrm{Glc}\right)$ and the indicated substrates. The assay was performed in the respective gel filtration buffer with $0.06 \mathrm{mM}$ substrate, $0.03 \mathrm{mM}$ UDP-Glc, $0.02 \mu \mathrm{Ci}$ UDP- ${ }^{14} \mathrm{C}(\mathrm{U})-\mathrm{Glc}$ for $1 \mathrm{~h}$ at $25{ }^{\circ} \mathrm{C}$. After metabolite extraction and thin layer chromatography (TLC), the TLC-plates were incubated on phosphor screens for $3 \mathrm{~d}$ and the radioactive signals were detected. Given are product signals minus background over signal area ((Signal-Bkg)/Area). UGT76E1, UGT76E2, UGT76E11, UGT76E12, and UGT74F1 were incubated with a set of different aglycones: hexadecanoic acid (16:0), $\omega$-hydroxy-16:0 ( $\omega-\mathrm{OH}-16: 0)$, 2-hydroxy-16:0 (2-OH-16:0), 3-hydroxy-16:0 (3-OH-16:0), hexadecanol (OH-C16), benzoic acid (BA), salicylic acid (SA), pipecolic acid (Pip), indole-3-caboxylic acid (ICA), abscisic acid (ABA), zeatin, gibberellic acid (GA), 12- 12-oxo-phytodienoic acid (12-OPDA), JA, 12-hydroxy-JA (12-OH-JA), 11 hydroxy-JA (11-OH-JA), 12-hydroxy-JA-methyl ester (12-OH-JA-ME), quercetin, dihydro-myricetin (dh-myricetin), dihydro-kaempferol (dh-kaempferol), and a control with inactivated enzyme (control). The data shows means of 3 independent experiments + SE for every UGT. 


\subsubsection{HOMOGENOUS ENZYMES CAN BE BROUGHT BACK TO THEIR NATIVE SUBSTRATES}

Besides single reactions (see 4.3.5), it is important to bring the UGTs in contact with a mixture of different substrates that may be available - like in a living cell. A new approach, which analyzes the enzymes in their native substrate environment, was adapted to the requirements of the four UGTs. To achieve this, enzyme biochemistry was combined with metabolomics; homogenous proteins were incubated with a metabolite rich extract from A. thaliana and measured with the non-targeted metabolite fingerprinting platform (Kaever, 2014). Differential analyses of metabolite rich extracts with and without enzyme can hereby identify specific reaction partners from a natural substrate mixture.

12-OH-JA-Ile may a putative jasmonate substrate of the UGTs since Kitaoka and co-workers, 2014, have found 12-O-Glc-JA-Ile in wounded A. thaliana (Kitaoka et al., 2014). So far, 12-OHJA-Ile could not be investigated by the former in vitro activity assays (see Figure 4-10) because the compound was not available. At the same time, it is well known that 12-OH-JA-Ile accumulates in response to wounding in vivo (Figure 4-18E) (Bruckhoff et al., 2016). Therefore, the biological material of a wounding experiment (see Figure 4-18) was used to provide substrates like 12-OH-JA and 12-OH-JA-Ile and mimic the native environment (see 4.1.3). Due to comprehensibility, attention was given to $2 \mathrm{hpw}$. At this time point, the substrate availability was best for UGT-activity towards jasmonates: 12-OH-JA and 12-OH-JA-Ile have accumulated and internal abundance of 12-O-Glc-JA is still low (Supplemental figure 14, see Figure 4-18).

Nevertheless, the ex vivo fingerprinting approach entails several variables due to the complexity of the system. For this reason, different controls were taken to distinguish between enzymatic activities and internal plant metabolites. On the one hand, a negative control (heat-inactivated enzyme) was used to determine substrates and internal features. On the other hand, a positive control was performed to inspect product formation by spiking known substrates into the reaction solutions. Both controls together were thought to provide strong substrate/product patterns to prove the activity of the UGTs and to confirm the existence of the respective substrates. Altogether, the experiment used metabolite rich plant extracts after wounding ( $2 \mathrm{hpw}$ ) including potential jasmonates substrates (12-OH-JA and 12-OH-JA-Ile) that were dissolved in protein buffer $(50 \mathrm{mM}$ Tris/HCl, $\mathrm{pH} 8,100 \mathrm{mM} \mathrm{NaCl})$. The extracts were incubated $\left(1 \mathrm{~h}, 25^{\circ} \mathrm{C}\right)$ with $100 \mu \mathrm{g}$ homogenous UGTs or inactive enzymes (negative control) and $0.1 \mathrm{mM}$ UDP-Glc as general co-substrate. A second set of reaction was performed as positive control with additional substrates spikes into the reactions. Afterwards, both sets were measured with the non-targeted LC-MS analysis and data were processed with the MarVis-tool (Kaever, 2014). This tool filters features from all reactions by their statistical distribution, clusters them by similar abundances, and identifies them by databank comparisons. The graphical output represents mean intensities of 
the features (heat-map representation), which were clustered as one-dimensional self-organizing map (1D-SOMs).

The data of the ex vivo metabolite fingerprinting approach for UGT76E1, UGT76E2, UGT76E11, UGT76E12 and UGT74F1 and the controls are given as 1D-SOM with vertical clusters illustrating feature-intensities for every enzyme and the neg. control (horizontal). Globally, both reaction sets of the internal reactions and the positive controls with additional substrates show similar accumulations. The Clusters $1-5$ are dominated by patterns specific for UGT76E1. Meaning, features in these clusters are accumulating in samples incubated with UGT76E1. Clusters 6-10 show specific patterns for different enzymes: cluster 6 sums up features, which could represent products of UGT76E11, cluster 7 summarizes a pattern of tentative UGT76E1product, cluster 9 is connected to tentative products of UGT76E12, and cluster 10 represents UGT74F1-related features. The majority of features in clusters $11-18$ seems neither to be related to tentative products nor substrates of the five UGTs. Clusters 19 and 20 show features specific for the additional substrates of the positive controls (Figure 4-11).

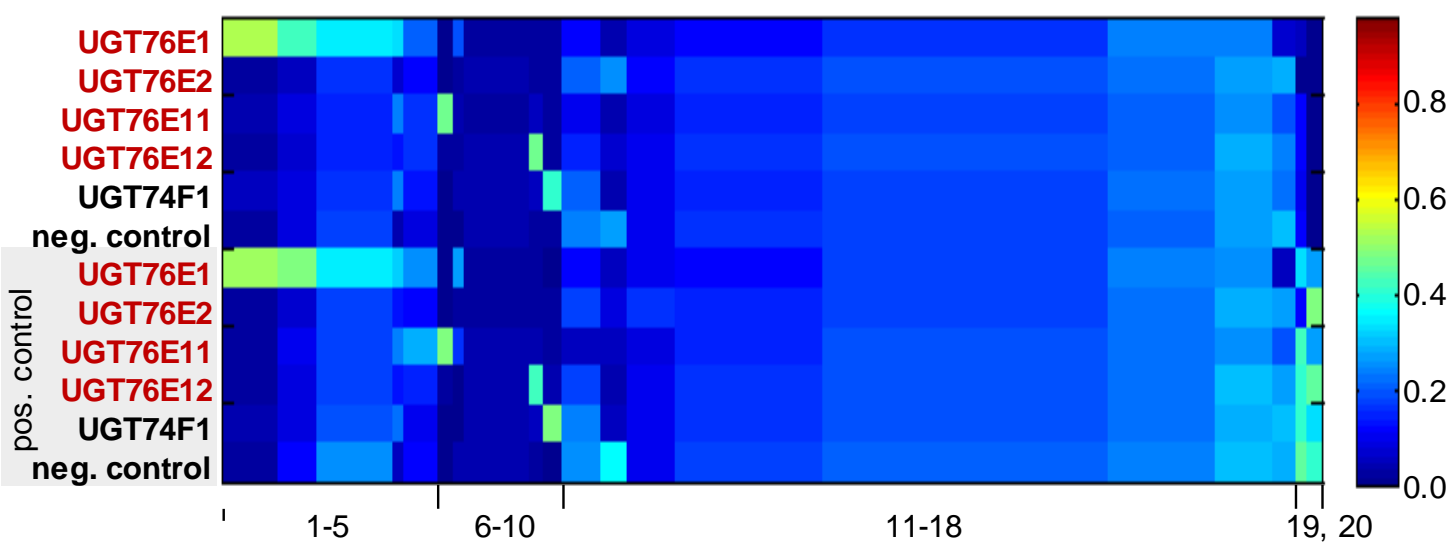

Figure 4-11: Search for native substrates of UGT76E1, UGT7E2, UGT76E11, UGT76E12, UGT74F1 by non-targeted $e x$ vivo analysis

For the enzymatic assay with the indicated UGTs total metabolite extracts of wounded A. thaliana plants were used. Therefore, plants were grown for six weeks at $22{ }^{\circ} \mathrm{C}$ under short day conditions $(8 \mathrm{~h} \mathrm{light} / 16 \mathrm{~h}$ dark). Leaves were wounded three times across the mid vein by squeezing with forceps. Damaged rosette leaves were harvested at 2 hours post wounding (pool of 10 plants per sample), extracted by two-phaseextraction, and used as substrate mix for the ex vivo activity assay. The extracts were resolved in $50 \mathrm{mM}$ Tris $\mathrm{pH} 8,100 \mathrm{mM} \mathrm{NaCl}$ buffer and incubated with $0.1 \mathrm{mM}$ UDP-Glc and $100 \mu \mathrm{g}$ of the indicated active UGT-enzyme or inactive enzymes (neg. control). As positive control (highlighted in grey), $0.01 \mathrm{mM}$ salicylic acid, $\omega$-hydroxy-hexadecanoic acid, and 12-hydroxy-JA were added to the mixtures. Reactions were incubated at $25^{\circ} \mathrm{C}$ for $1 \mathrm{~h}$, stopped by adding acetonitrile and analyzed by LC-MS-based metabolite fingerprinting. 1996 metabolite features $\left(\mathrm{pVal}<10^{-6}\right)$ were selected and their intensity profiles were clustered by means of one-dimensional self-organizing maps (1D-SOMs). Shown is the heat map representation of the clustering process by using 20 clusters. The number of features per cluster is proportional to the cluster-width. The data represents three measurements of one experiment that are representative for two independent experiments. Samples were measured by Dr. Kirstin Feussner (University of Goettingen, Germany). Experiment was designed and performed together with Dr. Kirstin Feussner. Data were analyzed with the MarVis tool (Kaever, 2014). 
To streamline the extraction of tentative substrates and products out of the 1996 features of the ex vivo data set three criteria for data analysis were established. Tentative pairs of product and substrate are characterized by: (i) an exact mass difference of a Glc moiety (162.016 Da), (ii) a RT-shift of the more hydrophilic product in comparison to the substrate and (iii) inverse intensity pattern to each other.

\subsubsection{UGT76E1, UGT76E2, UGT76E12, AND UGT74F1 SHOW ACTIVITY TOWARDS THEIR KNOWN SUBSTRATES}

The patterns of the positive controls were used to evaluate the activities of the UGTs and support former findings in a concurrence situation. The substrates $\omega-\mathrm{OH}-16: 0,12-\mathrm{OH}-\mathrm{JA}$ and SA were spiked with $10 \mu \mathrm{M}$ each into every reaction of the positive controls. Features of both, the spiked substrates and the respective products, were identified in accordance to the three criteria defined above (Figure 4-12). First, SA could be detected in all samples besides those incubated with UGT74F1; UGT74F1 used up SA completely. The levels of the corresponding product, 2-O-Glc$\mathrm{SA}$, are increased 2-fold in UGT76F1-treated samples exclusively. All other samples show 2-OGlc-SA levels, which are similar to the negative control and seem to be natural amounts of 2-OGlc-SA in the $A$. thaliana extracts $2 \mathrm{hpw}$. The substrate $\omega-\mathrm{OH}-16: 0$ was used as a control reaction for all UGTs. The amounts of $\omega-\mathrm{OH}-16: 0$ are reduced in UGTE76E1-, UGT76E11-, UGT76E12, and UGT74F1-treated samples compared to the negative control. On the product level, UGT76E12-treated samples shows the highest intensity of $\omega$-O-Glc-16:0. UGT76E1, UGT76E2, and UGT74F1 illustrate similar product intensities about half of that of UGT76E12. The lowest activity is given by UGT76E11 with 1/5 of the maximal value. For 12-OH-JA, UGTE76E1- and UGT76E2-treated samples show reduced amounts of the substrate with stronger reduction in the UGT76E2-samples. In accordance to that, UGT76E2-treated samples show the highest amount of the product 12-O-Glc-JA, followed by UGT76E1-treated samples and, to a much lower extent, UGT76E12-treated samples (Figure 4-12).

The positive controls of the ex vivo experiment confirm the specific activities of the UGTs in a concurrence situation and reflect the activity levels of previous experiments. Moreover, the three criteria for identifying activities for the indicated enzymes could be evaluated with specific substrate and product couples. 


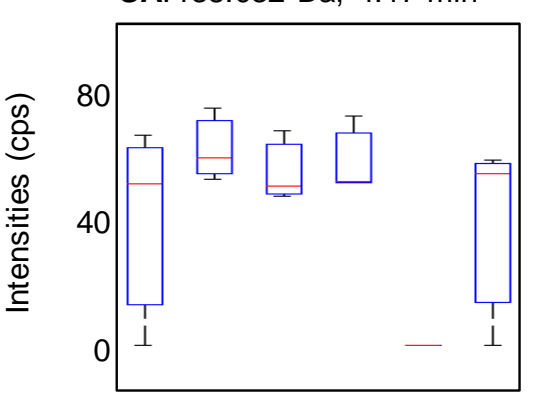

2-O-GIc-SA: $300.085 \mathrm{Da}, 2.72 \mathrm{~min}$

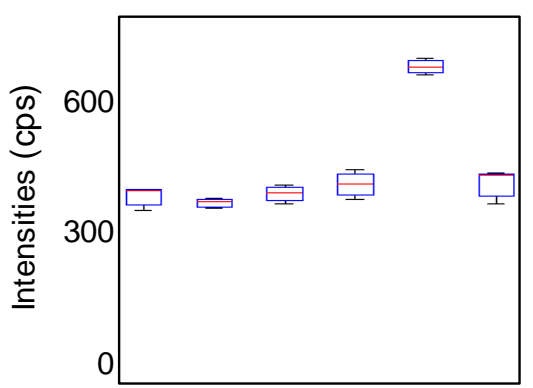

B)

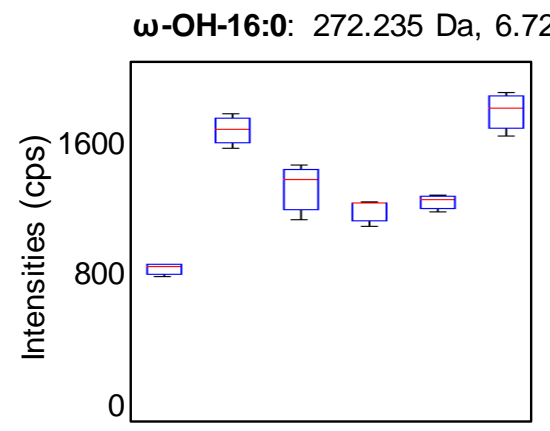

w-O-GIc-16:0: $434.297 \mathrm{Da}, 5.81 \mathrm{~min}$

C)

12-OH-JA: $226.121 \mathrm{Da}, 3.52 \mathrm{~min}$

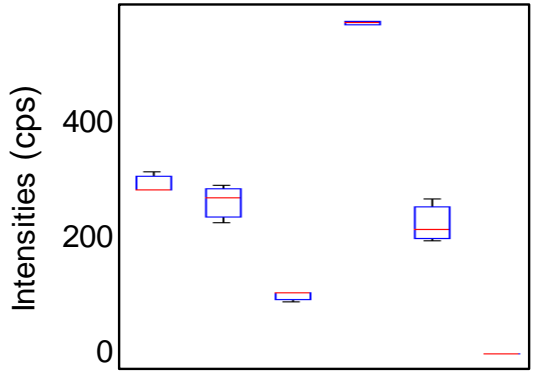

12-O-GIc-JA: $388.173 \mathrm{Da}, 3.24 \mathrm{~min}$
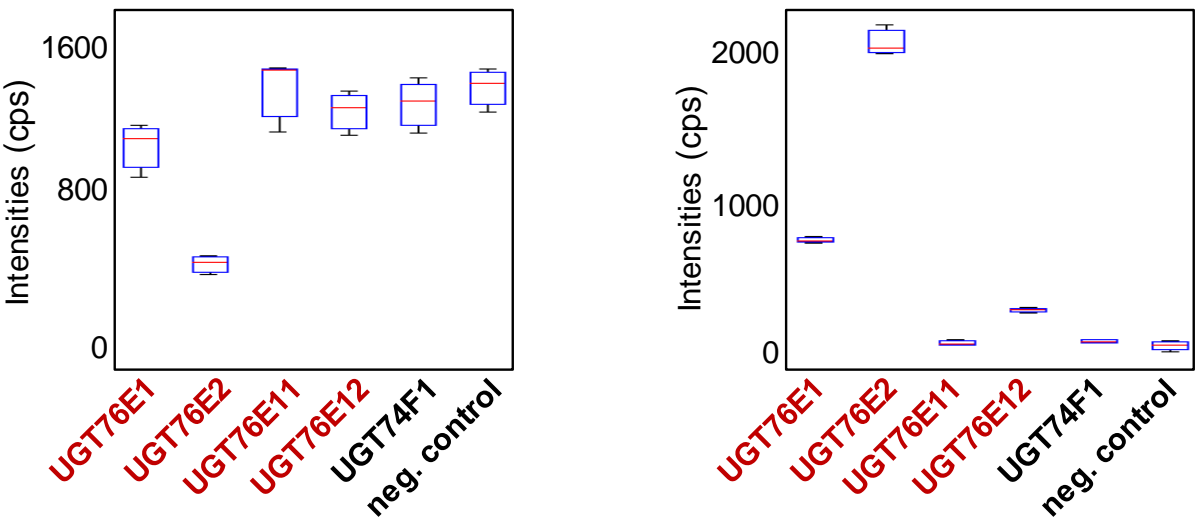

Figure 4-12: Activity of UGT76E1, UGT7E2, UGT76E11, UGT76E12, and UGT74F1 for 12-hydroxyJA, salicylic acid, and $\omega$-hydroxy-hexadecanoic acid in total metabolite extracts

For the enzymatic assays, total metabolite extracts of wounded $A$. thaliana plants were used. The indicated compounds were added as substrates and used as positive control for the ex vivo approach. Plants were grown for six weeks at $22{ }^{\circ} \mathrm{C}$ under short day conditions ( $8 \mathrm{~h}$ light/16 h dark). Leaves were wounded three times across the mid vein by squeezing with forceps. Damaged rosette leaves were harvested at 2 hours post wounding (pool of 10 plants per sample), extracted, and used as substrate mix for the ex vivo activity assay. The extracts were resolved in $50 \mathrm{mM}$ Tris $\mathrm{pH} 8,100 \mathrm{mM} \mathrm{NaCl}$ buffer and the assay was performed with $0.1 \mathrm{mM}$ UDP-Glc, $0.01 \mathrm{mM}$ salicylic acid, $0.01 \mathrm{mM} \omega$-hydroxyhexadecanoic acid, and $0.01 \mathrm{mM}$ 12-hydroxy-JA, $100 \mu \mathrm{g}$ of the indicated active UGT-enzyme or inactive enzyme (neg. control) for 1 hour at $25^{\circ} \mathrm{C}$. The reactions were stopped by adding acetonitrile and analyzed by LC-MS with a method, which was developed for non-targeted fingerprinting. Out of 1996 metabolite features with a $\mathrm{pVal}<10^{-6}$ the particular features for $\left.\mathbf{A}\right)$ salicylic acid (SA) and 2-O-glycosyl-SA (2-OGlc-SA), B) $\omega$-hydroxy-hexadecanoic acid ( $\omega$-OH-16:0) and $\omega$ - $O$-glucosyl-16:0 ( $\omega$-O-Glc-16:0), and C) 12-hydroxy-JA (12-OH-JA) and 12-O-glucosyl-JA (12-O-Glc-JA) are shown as Box-Whisker-plots with intensities given as counts per second (cps). The data represents three technical replicates of one experiment. Samples were measured by Dr. Kirstin Feussner (University of Goettingen, Germany). Experiment was designed and performed together with Dr. Kirstin Feussner. Data were analyzed with the MarVis tool (Kaever, 2014). 


\subsubsection{UGT76E1, UGT76E2, UGT76E11, UGT76E12, AND UGT74F1 SHOW ACTIVITY TOWARDS NATIVE SUBSTRATES}

The non-targeted ex vivo metabolite fingerprinting approach was initiated to bring back purified enzymes to their native substrate environment aiming to identify new substrates.

Two oxylipins were identified as new substrates of the four UGTs. The reaction pair of 13-HOT (276.210 Da, 6.59 min) and 13-glucosyl-O-octadecatrienoic acid (13-O-Glc-HOT, 465.274 Da, 5.65 min, Supplemental table 3) is shown in Figure 4-13A. There, UGT76E11 and UGT76E12 used up the substrate from the extracts and depict the highest product levels. UGT76E1 and UGT76E2-treated samples display half substrate levels and corresponding appr. $50 \%$ of the highest product intensity. UGT74F1-treated samples shows negative control patterns with highest substrate intensities and no product intensities (Figure 4-13A). Furthermore, the glycosylation of (7Z9E,13Z)-11-hydroxy-7,9,13-hexadecatrienoic acid (11-HHT, 266.186 Da, $6.08 \mathrm{~min}$ ) to 11-Oglycosyl-hexadecatrienoic acid (11-O-Glc-HHT, $428.240 \mathrm{Da}, 5.25 \mathrm{~min}$, Supplemental table 3) was identified. Here, UGT76E2, UGT76E11, and UGT76E12 form the high product levels. UGT76E1-treated samples showed about half of that activity and UGT74F1 did not tolerate this substrate with control-like intensities (Figure 4-13B). Both glycosylated oxylipins are not abundant in the extracts as indicated by the negative controls (see Figure 4-13A, B).

Next, the reaction pair of 12-OH-JA to $12-\mathrm{O}-\mathrm{Glc}-\mathrm{JA}$ was also identified as native reaction with substrate from the extracts. Here, UGT76E11, UGT76E12, and UGT74F1-treated samples show highest substrate levels corresponding to the accumulation of 12-OH-JA 2 hpw. UGT76E1treated samples illustrate slightly reduced amounts whereas UGT76E2 used up completely 12OH-JA from the extracts. 12-O-Glc-JA illustrates a complementary pattern: UGT76E11, UGT74F1, and the negative control display low intensities of the glycoside as present without enzymatic activity. UGT76E12 shows 3-fold, UGT76E1 6-fold, and UGT76E2 12-fold higher product levels (Figure 4-13C).

In addition, an unknown reaction pair for UGT76E11 was identified. The substrate shows no wound-related accumulation in the leaf extracts; it is detectable at 0,2 , and $5 \mathrm{hpw}$ (data not shown). The substrate feature has an exact mass of $198.126 \mathrm{Da}$, a RT of $5.47 \mathrm{~min}$ and a deduced sum formula of $\mathrm{C} 11 \mathrm{H} 18 \mathrm{O} 3$. UGT76E11 used up the substrate exclusively forming high amounts of the corresponding product (Glc-C11H18O3, 360.179 Da, RT 4.65 min). Glc-C11H18O3 shows trace intensities in UGT76E1, UGT76E2, UGT76E12, UGT74F1-treated samples, and the negative control (Figure 4-13D). To obtain structural information about the compound MS/MS analyses were performed. The fragmentation of the UGT76E11-specific product (Glc$\mathrm{C} 11 \mathrm{H} 18 \mathrm{O} 3)$ show a loss of a hexose moiety, which leads to the mass signal of the substrate of $[\mathrm{M}+\mathrm{H}]^{+} 199.099$ and confirms the finding of the ex vivo approach (Fig. 4-13). The MS/MS spectrum of the substrate $(\mathrm{C} 11 \mathrm{H} 18 \mathrm{O} 3)$ shows three serial losses of water, which suggests the 
A)

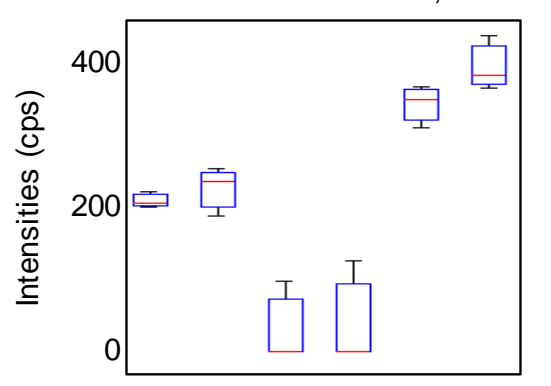

B)

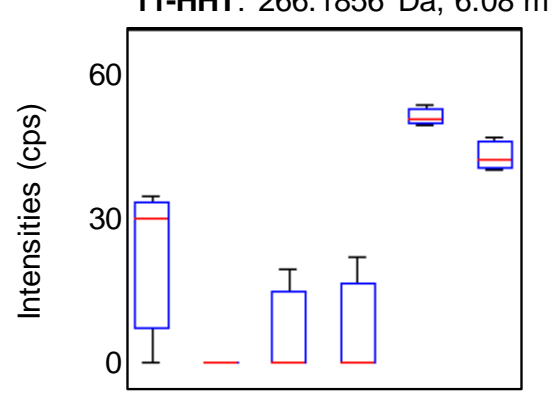

C)

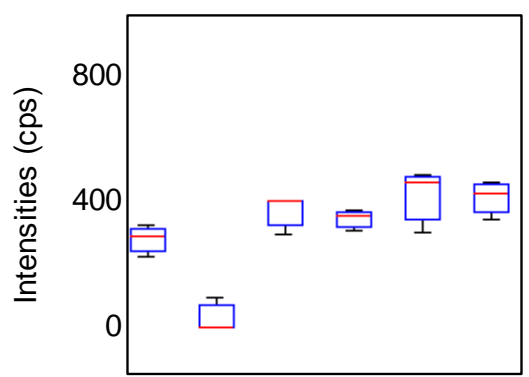

D)

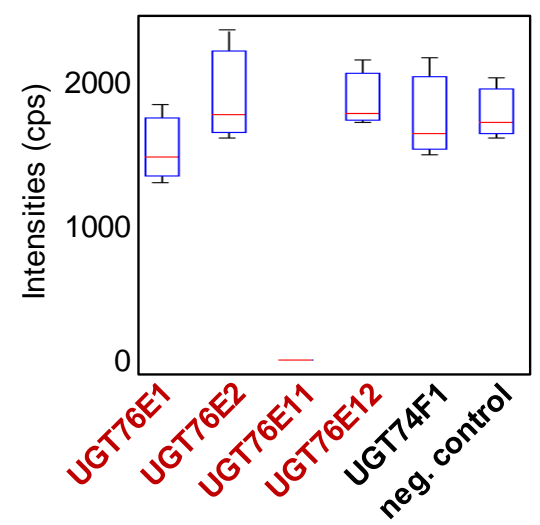

13-O-Glc-HOT: $456.274 \mathrm{Da}, 5.65 \mathrm{~min}$

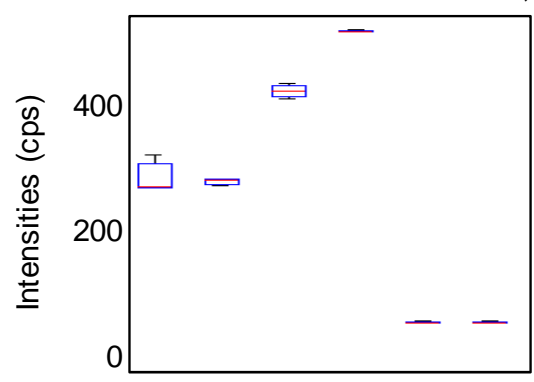

11-O-GIC-HHT: $428.240 \mathrm{Da}, 5.25 \mathrm{~min}$

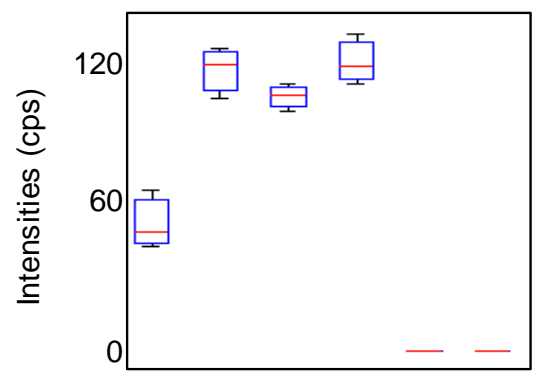

12-O-GIC-JA: $388.173 \mathrm{Da}, 3.24 \mathrm{~min}$

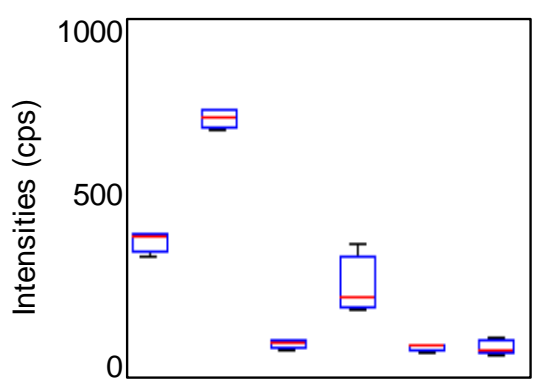

C11H1803-GIc: $360.179 \mathrm{Da}, 4.65 \mathrm{~min}$

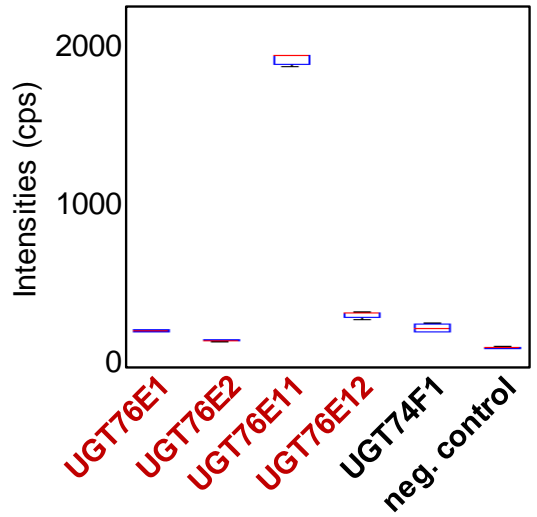

Figure 4-13: Native substrates of UGT76E1, UGT7E2, UGT76E11, UGT76E12, UGT74F1 identified by a non-targeted ex vivo analysis

For the enzymatic assays, total metabolite extracts of wounded $A$. thaliana plants were used. Plants were

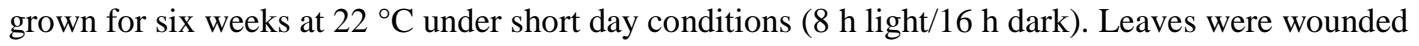
three times across the mid vein by squeezing with forceps. Damaged rosette leaves were harvested at 2 hours post wounding (pool of 10 plants per sample), extracted, and used as substrate mix for the ex vivo activity assay. The extracts were resolved in $50 \mathrm{mM}$ Tris $\mathrm{pH} 8,100 \mathrm{mM} \mathrm{NaCl}$ buffer and the assay was performed with $0.1 \mathrm{mM}$ UDP-Glc and $100 \mu \mathrm{g}$ of the indicated active UGT-enzyme or inactive enzyme (neg. control) for 1 hour at $25{ }^{\circ} \mathrm{C}$. The reactions were stopped by adding acetonitrile and analyzed by LCMS with a method, which was developed for non-targeted fingerprinting. Out of 1996 metabolite features 
with a pVal $<10^{-6}$ the particular features for A) 13-hydroxy-octadecatrienoic acid (13-HOT) and 13-Oglucosyl-octadecatrienoic acid (13-O-Glc-HOT), B) 11-hydroxy-hexadecatrienoic acid (11-HHT) and 11$O$-glycosyl-octadecatrienoic acid (11-O-Glc-HHT), C) 12-hydroxy-JA (12-OH-JA) and 12-O-glucosylJA (12-O-Glc-JA) and D) C11H18O3 and C11H18O3-Glc are shown as Box-Whisker-plots with intensities given as counts per second (cps). The data represents three technical replicates of one experiment. Samples were measured by Dr. Kirstin Feussner (University of Goettingen, Germany). Experiment was designed and performed together with Dr. Kirstin Feussner. Data was analyzed with the MarVis tool (Kaever, 2014).
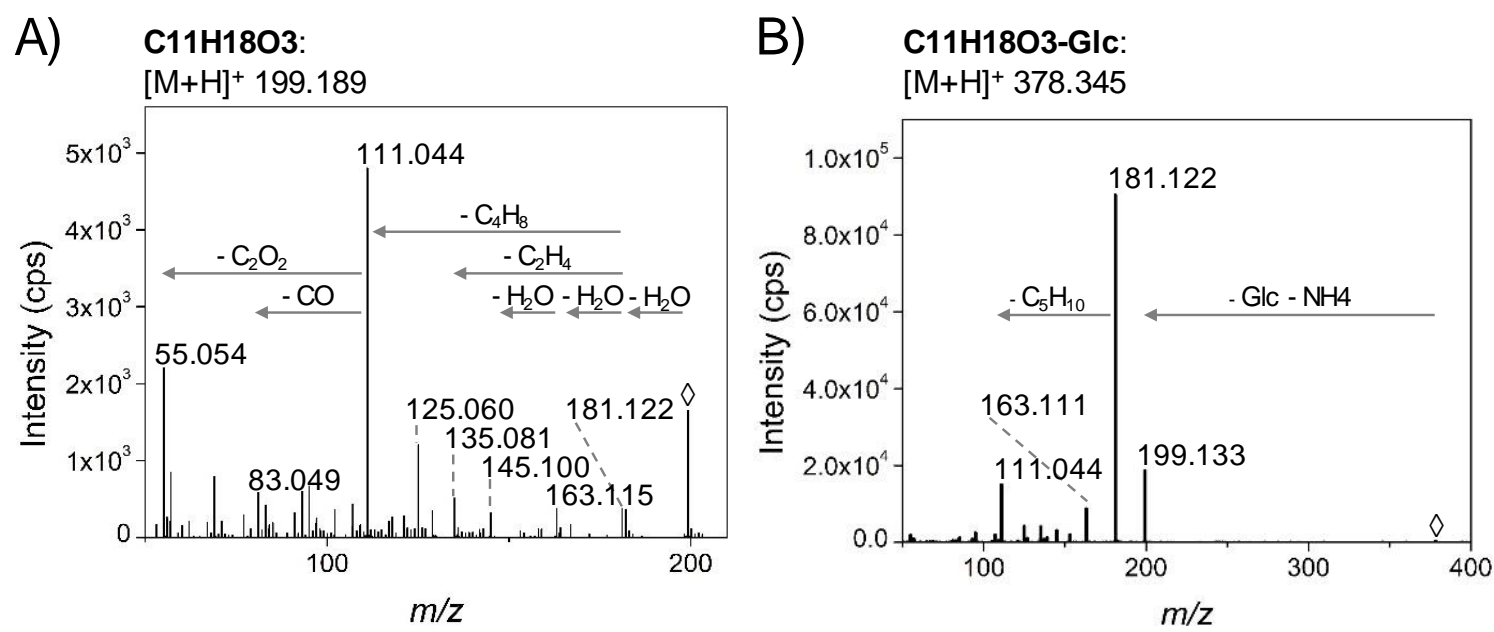

Figure 4-14: MS/MS analysis of the unknown substrate C11H18O3

The ex vivo metabolite fingerprinting experiment identified a reaction pair for UGT76E11: C11H18O3 to C11H18O3-Glc. LC-MS/MS analysis were performed with collision energies of $12 \mathrm{eV}$ in the positive ionization mode. MS/MS fragmentation of $\mathbf{A})$ the substrate signal $\left(\mathrm{C} 11 \mathrm{H} 18 \mathrm{O} 3,[\mathrm{M}+\mathrm{H}]^{+} 199.099\right.$, at 6.01 $\min )$ B) the product signal $\left(\mathrm{C} 11 \mathrm{H} 18 \mathrm{O} 3-\mathrm{Glc},[\mathrm{M}+\mathrm{H}]^{+}\right.$378.345at 5.01 $\left.\mathrm{min}\right)$. LC-MS/MS analysis was performed by Dr. Kirstin Feussner. Intensities given as counts per second (cps).

existence of three hydroxy groups. The main fragment of $[\mathrm{M}+\mathrm{H}]^{+} 111.044$ equals $\mathrm{C} 6 \mathrm{H} 7 \mathrm{O} 2$. The UGT76E11-specific substrate could not be elucidated in more detail. In summary, 13-HOT, 11HHT are new substrates for UGT76E1, UGT76E2, UGT76E11, and UGT76E12. All four enzymes formed the respective products. Furthermore, the high activity of UGT76E2 towards 12OH-JA is supported by the ex vivo approach, too. In addition, UGT76E1 and UGT76E12 again show activity towards 12-OH-JA. Here, a first hypothesis for a specific, but so far not structurally elucidated substrate for UGT76E11 was generated.

\subsubsection{12-OH-JA-ILE IS NO SUBSTRATE FOR THE ANALYZED UGTS}

Since 12-OH-JA-Ile was identified in A. thaliana after wounding (Kitaoka et al., 2014), the potential glycosylation reaction of $12-\mathrm{OH}-\mathrm{JA}-\mathrm{Ile}$ to $12-\mathrm{O}$-Glc-JA-Ile was of special interest in this study. Because 12-OH-JA-Ile was abundant in the ex vivo extracts after wounding (see Supplemental figure 14, $f f$. Figure 4-18E), it was likewise tested as potential substrate of the different candidate UGTs. By the criteria introduced above, a putative product was searched by the exact mass of 501.257 Da and an earlier RT than 4.9 min (12-OH-JA-Ile). However, a feature of this mass could not be identified in any of the ex vivo samples (see Supplemental figure 14). 
Meaning, neither UGT76E1, UGT76E2, UGT76E11, UGT76E12, nor UGT74F1 tolerate 12-OHJA-Ile for catalysis. This finding was supported by a LC-HR-MS analysis of the UGT76E2treated sample (2 hpw) as second layer of evidence (Supplemental figure 14). Noticeably, these measurements also imply that $12-O$-Glc-JA-Ile is not present as internal metabolite of $A$. thaliana with or without wounding stimulus here.

Worth mentioning, 12-COOH-JA was detected the first time in wounded leaves of A. thaliana here. Before, the metabolite has been described in flowers of $A$. thaliana exclusively (Bruckhoff et al., 2016). From the metabolite rich extracts of the ex vivo approach, it was possible to identify the compound by exact mass and RT in the positive as well in the negative ionization mode. The pattern of 12-COOH-JA shows late wound accumulation at $5 \mathrm{hpw}$ and its identity was confirmed by LC-MS/MS fragmentation (Supplemental figure 13).

\subsubsection{SUBSTRATE PREFERENCES OF UGT76E1, UGT76E2, UGT76E11, UGT76E12, AND UGT74F1}

So far, the characterization of the enzymes UGT76E1, UGT76E2, UGT76E11, and UGT7612 has given clear substrate preferences but has also indicated additional (side) activities. Therefore, a LC-MS-based activity assay was performed to judge the performances. UGT76E1, UGT76E2, UGT76E11, UGT76E12, and UGT74F1 were tested with the substrates $\omega$-OH-16:0, SA, 12-OHJA, 9-HOT, 13-HOT. Additionally, (10E,15Z)-9,12,13-trihydroxy-10,15-octadecadienoic acid (triOH-18:2) and (10E)-9,12,13-trihydroxy-10-octadecaenoic acid (triOH-18:1) were included. It should be tested, if the UGTs would tolerate up to three hydroxyl-groups on a fatty acid as a substrate and if several glycosylations could be performed with one substrate molecule.

$\omega-\mathrm{OH}-16: 0$ is preferably glycosylated by UGT76E12 (100\%). In comparison to that UGT76E2 shows $36 \%$, UGT76E1 $9 \%$, UGT74F1 $4 \%$, and UGT76E11 illustrates $2 \%$ relative conversion rate. Similarly, both oxylipins, 9-HOT and 13-HOT, are best substrates of UGT76E12. Anyhow, 9-O-Glc-HOT and 13-O-Glc-HOT are also formed by UGT76E2 $(22 \%, 94 \%)$ and UGT76E11 $(5 \%, 98 \%)$. UGT76E1 produces low amounts of 13-O-Glc-HOT (6\%) but no 9-O-Glc-HOT. SA is the native substrate of UGT74F1 and 2-O-Glc-SA is exclusively formed by this enzyme (100 \%). 12-O-Glc-JA is formed from 12-OH-JA by UGT76E2 (100 \%), UGT76E1 (29\%), and UGT76E12 (28\%). The tri-hydroxy fatty acids were detected in the samples but neither mono-, di, nor tri-glycosylated products could be identified for any of the enzymes (Table 4-4).

Altogether, the evaluation of the substrate preferences supports the specificities and tolerances observed before: UGT76E2 glycosylates preferentially 12-OH-JA. In addition, it uses 13-HOT with a high relative conversion rate as well as $\omega-\mathrm{OH}-16: 0$ and 9-HOT to lower extent. UGT76E1 forms $12-O$-Glc-JA very specifically, but with a lower conversion rate as UGT76E2. UGT76E12 shows broader substrate tolerance with highest activities towards the hydroxyl fatty acids and to 
a lower extent with 12-OH-JA. UGT76E11 depicts high relative conversion rates with 13-HOT.

None of the candidates tolerated tri-hydroxy-oxylipins as substrates.

Table 4-4: Relative substrate preference of UGT76E1, UGT76E2, UGT76E11, UGT76E12, and UGT74F1

Substrates so far identified for UGT76E1, UGT76E2, UGT76E11, UGT76E12, and UGT74F1 are evaluated by an in vitro assay. The relative conversion rate (\%) was analyzed by LC-MS: $\omega$-hydroxyhexadecanoic acid ( $\omega$-OH-16:0), salicylic acid (SA), 12-hydroxy-JA (12-OH-JA), 9-hydroxyoctadecatrienoic acid (9-HOT), 13-hydroxy-octadecatrienoic acid (13-HOT). In addition, 9,12,15trihydroxy-octadecadienoic acid (tri-OH-18:2) and 9,12,13-trihydroxy-octadecaenoic acid (tri-OH-18:2 were tested as substrates. In a $100 \mu \mathrm{L}$ reaction assay, $10 \mu \mathrm{g}$ homogenous enzyme were incubated with $0.05 \mathrm{mM}$ substrate and $0.25 \mathrm{mM}$ UDP-Glc at $25^{\circ} \mathrm{C}$ for $30 \mathrm{~min}$. The reactions were stopped with $100 \mu \mathrm{L}$ ACN. n. d., not detected. The data are representative for one experiment.

\begin{tabular}{c|ccccc}
\hline Substrate & UGT76E1 & UGT76E2 & UGT76E11 & UGT76E12 & UGT74F1 \\
\hline$\omega$-OH-16:0 & 9 & 36 & 2 & 100 & 4 \\
SA & n. d. & n. d. & n. d. & n. d. & 100 \\
12-OH-JA & 29 & 100 & n. d. & 28 & n. d. \\
9-HOT & n. d. & 22 & 5 & 100 & n. d. \\
13-HOT & 6 & 94 & 98 & 100 & n. d. \\
Tri-OH-18:1 & n. d. & n. d. & n. d. & n. d. & n. d. \\
Tri-OH-18:2 & n. d. & n. d. & n. d. & n. d. & n. d. \\
\hline
\end{tabular}

\subsubsection{The Kinetic Parameters of UGT76E1, UGT76E2, UGT76E11, AND UGT76E12 WERE ESTIMATED BY A COUPLED PHOTOMETRIC}

A continuous record of an enzymatic reaction allows determining the initial linear phase, a socalled steady-state kinetic. The initial velocity of a reaction is often measured by a spectrophotometric assay. Since the compounds of the UGT-reaction do not change their photometrical properties upon catalysis, the reaction was coupled to a redox reaction, which includes nicotinamide adenine dinucleotide $\left(\mathrm{NADH} / \mathrm{NAD}^{+}\right)$as reaction partner. In particular, UGTs release UDP as co-product, which ( $c f$. Reaction 1) can be linked to pyruvate kinase (PK) and lactate dehydrogenase (LDH) to record the UGT reaction kinetics in $1: 1: 1$ stoichiometry. The PK natively phosphorylates ADP to ATP by metabolizing phosphoenolpyruvate (PEP) to pyruvate. However, it is also capable to phosphorylate UDP to UTP. Next, LDH catalyzes the reduction of pyruvate to lactate by oxidizing NADH to NAD ${ }^{+}$. NADH shows an absorption maximum at $340 \mathrm{~nm}$, which $\mathrm{NAD}^{+}$does not. (Figure 4-15). Hence, the UGT reaction is indirectly monitored by the decrease of the absorption of NADH at $340 \mathrm{~nm}$ and the reaction velocities can be determined from the initial slope (Figure 4-15, Equation 5) (cf. Brown et al., 2012). Like this, the steady-state kinetics of UGT76E1, UGT76E2, UGT76E11, and UGT76E12 were determined towards 9-HOT and 13-HOT. Additionally, UGT76E1 and UGT76E2 were measured with their preferred substrate 12-OH-JA. Due to limitations in the availability of 12-OH-JA, the kinetic 


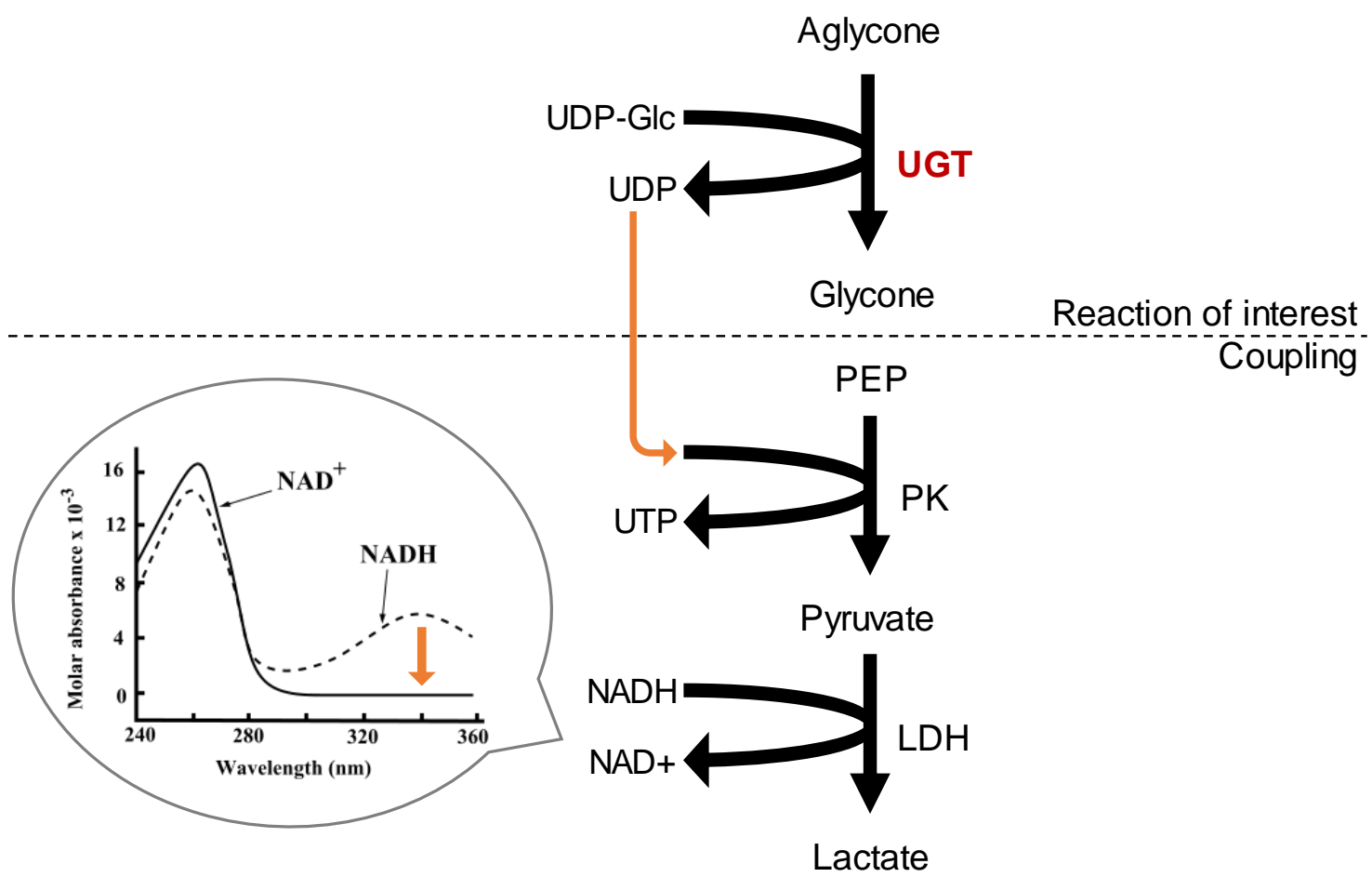

Figure 4-15: Scheme of a coupled spectrophotometric assay to record reactions of uridine diphosphate-dependent glycosyltransferases

Uridine diphosphate (UDP)-dependent glycosyltransferases (UGTs) transfer a glucose (Glc) moiety from UDP-Glc to the hydroxyl-aglycone releasing the respective glycone and UDP. The UGT-reaction has been coupled via the co-product UDP to the pyruvate kinase (PK) and the lactate dehydrogenase (LDH) to record the UGT reaction kinetics in $1: 1: 1$ stoichiometry: The PK phosphorylates UDP to UTP while metabolizing phosphoenolpyruvate (PEP) to pyruvate. LDH catalyzes the reduction of pyruvate to lactate by oxidizing NADH to NAD ${ }^{+}$, which shows no absorption maximum at $340 \mathrm{~nm}$. The assay was adapted from Brown et al., 2012. The spectrum was taken from https://en.wikipedia.org/wiki/Nicotinamide_adenine_dinucleotide.

parameters of UGT76E12 against 12-OH-JA could not be obtained. In addition, the control enzyme UGT74F1 was measured with SA. First, it must be shown that the coupling reactions (PK- and LDH-reaction) are not rate limiting for determining the reaction parameters of the reaction of interest. Therefore, a positive control ( $450 \mu \mathrm{M}$ UDP) illustrates the capacity of the system. Generally, all UGT-reactions started with a lack phase. Then, the linear reaction phase steady-state conditions - lasted for at least $100 \mathrm{~s}$ (UGT76E2 with $500 \mu \mathrm{M}$ 12-OH-JA). The setup recorded specific activities at different substrate concentrations. The respective values of the steady-state slopes were plotted as reaction rate per enzyme concentration $(\mathrm{V} /[\mathrm{E}])$ against the substrate concentration.

For the kinetic analyses of UGT76E1, $10 \mu \mathrm{g}$ of the enzyme were stabilized with BSA and 12OH-JA was used as substrate in a concentration range from $0-1179 \mu \mathrm{M}$. UGT76E1 showed only little initial reaction velocities, even in the range of substrate saturation at $>393 \mu \mathrm{M}(\Delta$ $0.02 \mathrm{AU}$ per min). Overall, the conversion of 12-OH-JA to 12-O-Glc-JA by UGT76E1 follows the Michaelis-Menten model (Figure 4-16). The reaction rate, which were obtained for $262 \mu \mathrm{M}$ 12-OH-JA do not match well with the Michaelis-Menten fit, but the fit still shows a correlation 
of 0.982 (Table 4-5). The calculated parameters are the Michaelis-Menten constant $\left(\mathrm{K}_{\mathrm{M}}\right)$ of $61 \mu \mathrm{M}$ 12-OH-JA and a turnover number $\left(\mathrm{k}_{\mathrm{cat}}\right)$ of $0.0123 \mathrm{~s}^{-1}$. Before, UGT76E1 has shown low activity rates, which could be resolved here: UGT76E1 shows high catalytic affinity towards the preferred substrate 12-OH-JA $(61 \mu \mathrm{M})$ but it is characterized as a slow enzyme $\left(\mathrm{k}_{\text {cat }}\right.$ of $\left.0.0123 \mathrm{~s}^{-1}\right)$. Since the affinity of UGT76E1 to 12-OH-JA is high, it is most likely that the low turnovers of UGT76E1

A)

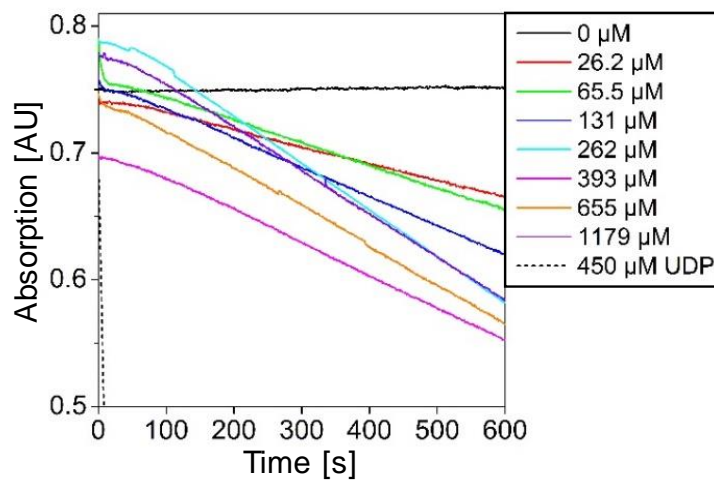

B)

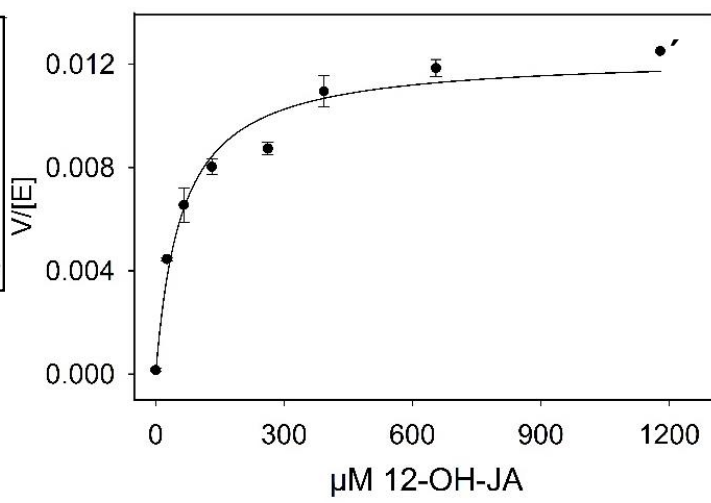

Figure 4-16: Enzyme kinetics of UGT76E1 with 12-hydroxy-JA

The initial velocities of UGT76E1-reaction were determined by a coupled spectrophotometric assay via the co-product UDP to pyruvate kinase and lactate dehydrogenase. The assay was adapted from Brown $e t$ al., 2012, and recorded at $340 \mathrm{~nm}$. The reactions were performed at $25^{\circ} \mathrm{C}$ with $10 \mu \mathrm{g}$ UGT76E1 (stabilized with $0.13 \%$ [w/v] bovine serum) in $50 \mathrm{mM}$ Tris/HCl, $\mathrm{pH}$ 9.0, $100 \mathrm{mM} \mathrm{NaCl}, 0.5 \mathrm{mM}$ UDPGlc, and the indicated concentrations of 12-hydroxy-JA (12-OH-JA). A) The initial slopes of the UGT76E1-reactions are shown. The data shows one dataset of three biological replicates. B) MichaelisMenten plot of the UGT76E1-reactions. The data are mean values with standard deviation of three biological replicates. Due to limitations in 12-OH-JA availability, the value for $1179 \mu \mathrm{M}$ was measured only once.

are caused by restrictive conditions. Likewise, not optimal buffer conditions will influence the kinetic parameters similar to a competitive inhibition resulting in lower turnovers $\left(\mathrm{k}_{\mathrm{cat}}\right)$ but unchanged affinity $\left(\mathrm{K}_{\mathrm{M}}\right)$.

Next, UGT76E2 was measured with 12-OH-JA. In contrast to UGT76E1, UGT76E2 was not stabilized with BSA and shows much higher activities. The reaction rate of UGT76E2 at $500 \mu \mathrm{M}$ 12-OH-JA was appr. 100 times faster than that of UGT76E1 (Figure 4-17). The same holds true for the absolute signals: at appr. $500 \mu \mathrm{M}$ 12-OH-JA, UGT76E2 is around 20 -fold faster $(\Delta$ $0.300 \mathrm{AU}$ per min) than UGT76E1 ( $\Delta 0.015 \mathrm{AU}$ per min). The values of the UGT76E2-kinetic follow the Michaelis-Menten model and can be fitted (Equation 5) with 0.998 correlation (Table 4-5). Noticeably, the reaction rate does still not show saturation at the highest substrate concentration (500 $\mu \mathrm{M}$ 12-OH-JA). Anyhow, UGT76E2 shows a $\mathrm{K}_{\mathrm{M}}$ of $219 \mu \mathrm{M}$ for 12-OH-JA and a $\mathrm{k}_{\mathrm{cat}}$ of $1.48 \mathrm{~s}^{-1}$ (Table 4-5). Here, the specific affinity of UGT76E2 and 12-OH-JA is 3-fold higher than for UGT76E1 but, at the same time, the turnovers are 100-fold faster. Those 
parameters seem to reflect the high activities observed in the former experiments and confirms the specificity and preference of UGT76E2 towards 12-OH-JA.

A)

B)
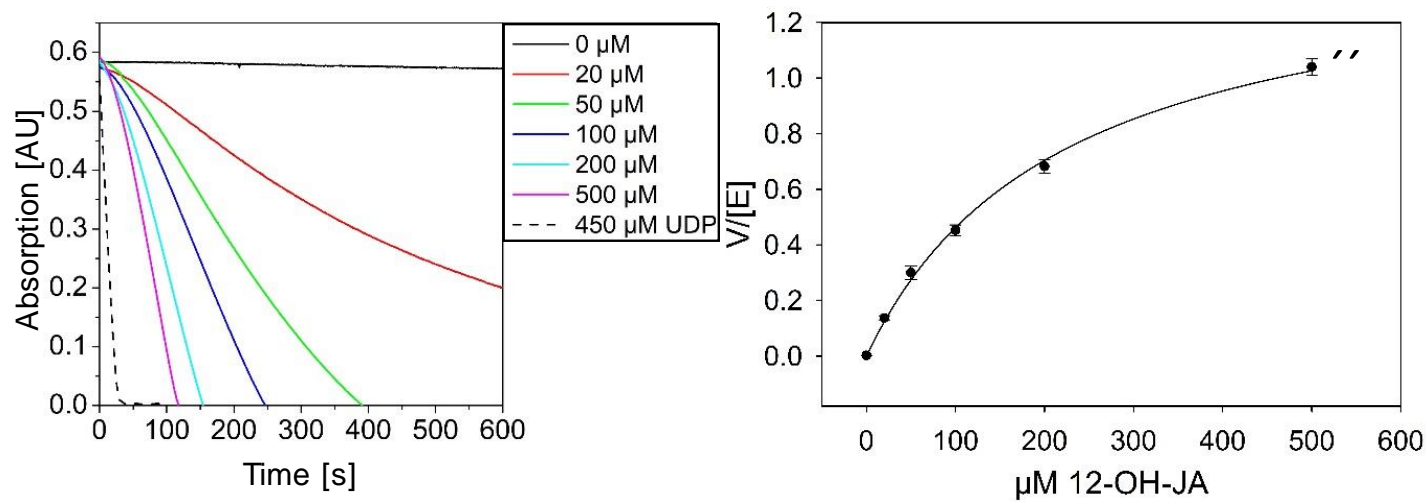

Figure 4-17: Enzyme kinetics of UGT76E2 with 12-hydroxy-JA

The initial velocities of UGT76E2-reaction were determined by a coupled spectrophotometric assay via the co-product UDP to pyruvate kinase and lactate dehydrogenase. The assay was adapted from Brown $e t$ al., 2012, and recorded at $340 \mathrm{~nm}$. The reactions were performed at $25^{\circ} \mathrm{C}$ with $5 \mu \mathrm{g}$ UGT76E2 in $50 \mathrm{mM}$ Tris/HCl, pH 8.0, $100 \mathrm{mM} \mathrm{NaCl}, 0.5 \mathrm{mM}$ UDP-Glc, and the indicated concentrations of 12-hydroxy-JA (12-OH-JA). A) The initial slopes of the UGT76E2-reactions are shown. The data shows one dataset of three biological replicates. B) Michaelis-Menten plot of the UGT76E2-reactions. The data are mean values with standard deviation of three biological replicates. Due to limitations in 12-OH-JA availability, the value for $500 \mu \mathrm{M}$ was measured only twice.

Furthermore, the kinetic parameters for UGT76E1, UGT76E2, UGT76E11, and UGT76E12 with the substrates 9-HOT and 13-HOT were determined. 13-HOT was found to be a general substrate of all four UGTs (see Figure 4-11). In case of 9-HOT, no enzymatic activities could be detected for UGT76E1, UGT76E2, and UGT76E11 (Table 4-5). For UGT76E12, a very high affinity with a $\mathrm{K}_{\mathrm{M}}$ of $21 \mu \mathrm{M}$ but at the same time a very low turnover with $\mathrm{k}_{\mathrm{cat}}$ of $0.0007 \mathrm{~s}^{-1}$ was observed towards 9-HOT (Table 4-5, Supplemental figure 15A). For 13-HOT, it was possible to record the kinetic parameters of UGT76E2, UGT76E11, and UGT76E12. Just UGT76E1 did not shown detectable activities towards 13-HOT (Table 4-5). For UGT76E2, the kinetic measurements displays linear correlation between reaction velocity and substrate concentrations and no saturation (Supplemental figure 15B). However, the fit to the Michaelis-Menten-model showed a correlation of 0.951 , which allows to calculate the parameters for $\mathrm{K}_{\mathrm{M}}$ of $4 \mathrm{mM}$ and $\mathrm{k}_{\text {cat }}$ of $0.5 \mathrm{~s}^{-1}$ (Table 4-5,). The fit extrapolates the saturation resulting in 100-fold higher $\mathrm{K}_{\mathrm{M}}$ for 13-HOT with UGT76E2. UGT76E11 shows specific but very slow activity towards 13-HOT ( $\mathrm{K}_{\mathrm{M}}$ of $156 \mu \mathrm{M}$, $\mathrm{k}_{\text {cat }}$ of $0.0007 \mathrm{~s}^{-1}$, Table 4-5, Supplemental figure 15C). UGT76E12 illustrates very high specificity towards 13 -HOT but also the slowest turnovers of all kinetics. Nevertheless, the parameters were calculated with $\mathrm{K}_{\mathrm{M}}$ of $23 \mu \mathrm{M}$ and $\mathrm{k}_{\text {cat }}$ of $0.0005 \mathrm{~s}^{-1}$ (Table 4-5, Supplemental figure 15D). Taken together, the kinetics of UGT76E11 and UGT76E12 with the oxylipin 
substrates show a relative high affinity whereas the turnover rates are extremely low and at the detection limit of the spectrophotometric assay.

The control enzyme UGT74F1 was measured with its native substrate SA. The kinetic parameters were calculated with $\mathrm{K}_{\mathrm{M}}$ of $212 \mu \mathrm{M}$ and $\mathrm{k}_{\mathrm{cat}}$ of $0.0027 \mathrm{~s}^{-1}$ (Table 4-5, Supplemental figure 15E). This kinetic was recorded to relate the kinetics of the candidates to published data of other UGTs of A. thaliana. This $\mathrm{K}_{\mathrm{M}}$ matches well with the published data and this turnover rate is one order of magnitude lower than the former value (Table 5-1) (Lim et al., 2002). Since the kinetic parameters of the four candidate UGTs show similar values, the control measurements show, that the coupled spectrometric assay is suitable for the estimation of the kinetic parameters.

Table 4-5: Kinetic parameters of the UGT76E1, UGT76E2, UGT76E11, UGT76E12, and UGT74F1 towards their best substrates

Steady-state kinetics were recorded for all four UGTs with the common substrates 9hydroxyoctadecatrienic acid (9-HOT), 13- hydroxy-octadecatrienoic acid. (13-HOT). UGT76E1 and UGT76E2 were also tested towards 12-hydroxy-JA (12-OH-JA). UGT74F1 was measured with salicylic acid (SA). A coupled spectrophotometric assay with the pyruvate kinase and the lactate dehydrogenase was used to measure the reactions of the UGTs in a $1: 1: 1$ stoichiometry by the decrease of NADH at $340 \mathrm{~nm}$ (Brown et al., 2012). The data were fitted with the hyperbolic function and the correlations are given. The kinetic parameters were calculated as Michaelis-Menten constant $\left(\mathrm{K}_{\mathrm{M}}\right)$, the turnover rate $\left(\mathrm{k}_{\mathrm{cat}}\right)$, and the catalytic efficiency $\left(\mathrm{k}_{\mathrm{cat}} / \mathrm{K}_{\mathrm{M}}\right)$. The data is representative for three biological replicates of one experiment.

\begin{tabular}{lccccc}
\hline & Substrate & Correlation & $\mathbf{K}_{\mathbf{M}}(\boldsymbol{\mu M})$ & \multicolumn{1}{c}{$\mathbf{k}_{\text {cat }}\left(\mathbf{s}^{\mathbf{- 1}}\right)$} & $\mathbf{k}_{\mathbf{c a t}} / \mathbf{K}_{\mathbf{M}}\left(\mathbf{s}^{\mathbf{- 1}} \boldsymbol{\mu} \mathbf{M}^{-\mathbf{1}}\right)$ \\
\hline \multirow{2}{*}{ UGT76E1 } & 12-OH-JA & 0.982 & 61 & 0.0123 & $2.02 \cdot 10^{-4}$ \\
& 9-HOT & - & n. d. & n. d. & - \\
& 13-HOT & - & n. d. & n. d. & - \\
\hline \multirow{2}{*}{ UGT76E2 } & 12-OH-JA & 0.998 & 219 & 1.4770 & $67.44 \cdot 10^{-4}$ \\
& 9-HOT & - & n. d. & n. d. & - \\
& 13-HOT & 0.951 & 4000 & 0.5000 & $1.25 \cdot 10^{-4}$ \\
\multirow{2}{*}{ UGT76E11 } & 9-HOT & - & n. d. & n. d. & - \\
& 13-HOT & 0.993 & 156 & 0.0007 & $0.04 \cdot 10^{-4}$ \\
\multirow{2}{*}{ UGT76E12 } & 9-HOT & 0.983 & 21 & 0.0007 & $0.33 \cdot 10^{-4}$ \\
& 13-HOT & 0.990 & 23 & 0.0005 & $0.22 \cdot 10^{-4}$ \\
\hline \multirow{2}{*}{ UGT74F1 } & SA & 0.998 & 212 & 0.0027 & $0.13 \cdot 10^{-4}$ \\
\hline
\end{tabular}

In summary, the coupled spectrophotometric assay enabled kinetic investigations of UGT76E1, UGT76E2, UGT76E11, UGT76E12, and the control UGT74F1. UGT76E1, UGT76E11, UGT76E12, and UGT74F1 turned out to be slow catalysts with very poor turnover rates (Table 4-5). In this regard, the compromise of three enzymatic reactions coupled might reduce the performance of the UGTs. Exclusively, UGT76E2 show better $\mathrm{k}_{\text {cat }}$-values with 12-OH-JA and 13-HOT (Table 4-5). On the other side, the specific affinities of the candidate UGTs were comparable to UGT74F1 (Table 4-5). Thus, the calculated $\mathrm{K}_{\mathrm{M}^{-}}$values of UGT76E1 and UGT76E2 for 12-OH-JA strengthen the hypothesis that these are specific 12-OH-JA UGTs. 


\subsection{JASMONATES ACCUMULATE AFTER WOUNDING}

The metabolite patterns of different jasmonates after wounding are well known (Bruckhoff et al., 2016; Mosblech, 2010). Nevertheless, it was important for this study to ensure a stable and functional wound response in A. thaliana. The wounded plant material was the resource for the quantitative expression data and the ex vivo metabolite fingerprinting approach (see 4.1.4 and 4.3.6). Therefore, the wounding experiment was set up like in Mosblech, 2010 and harvested at $0,1,2$, and $5 \mathrm{hpw}$. The samples were analyzed by the UPLC-ESI-MS/MS-based phytohormone profiling (Iven et al., 2014). Quantitative data are calculated from authentic internal standards given in nmol/g fresh weight (f. w.). Analytes, for which authentic standards were not available, relative intensities (p.d.u.) related to the chemically most similar standard are given.

JA and the active compound JA-Ile rise to maximal values at $1 \mathrm{hpw}(\mathrm{JA} 3.1 \mathrm{nmol} / \mathrm{g} \mathrm{f.} \mathrm{w.,} \mathrm{JA-Ile}$ $0.9 \mathrm{nmol} / \mathrm{g} \mathrm{f}$. w.) and decrease within the next $4 \mathrm{~h}$ (Figure 4-18A, D). The oxidized jasmonates, 11/12-hydroxy-JA (11/12-OH-JA), 12-OH-JA-Ile and 12-COOH-JA-Ile accumulate after wounding with highest values at $5 \mathrm{hpw}$. The amount of 11/12-OH-JA is enriched to $2.4 \mathrm{nmol} / \mathrm{g}$ f. w. at $5 \mathrm{hpw}$ (Figure 4-18B). 12- $\mathrm{HSO}_{4}-\mathrm{JA}$ and 12-O-Glc-JA are both formed from 12-OH-JA. 12- $\mathrm{HSO}_{4}-\mathrm{JA}$ accumulates after wounding considerably but not significantly (Figure 4-18C), while 12-O-Glc-JAaccumulates significantly 2.5 -fold at 2 and 6-fold at 5 hpw (Figure 4-18G).

Similar to jasmonates, the precursor 12-OPDA displays significant accumulations at $1 \mathrm{hpw}$ ( $2 \mathrm{nmol} / \mathrm{g} \mathrm{f.} \mathrm{w.)} \mathrm{and} \mathrm{at} 2 \mathrm{hpw}(3 \mathrm{nmol} / \mathrm{g} \mathrm{f.} \mathrm{w}$.) and reduced levels at $5 \mathrm{hpw}(1.3 \mathrm{nmol} / \mathrm{g} \mathrm{f}$. w.) (Supplemental figure 16). Additionally, the wounding time course for SA and ABA, their glycosylated forms, 2-O-Glc-SA and ABA-GE as well as IAA and ICA are shown in Supplemental figure 16. This wounding experiment shows a functional wound response of A. thaliana. Hence, the biological material tested here was suitable for the quantitative expression analysis and the non-targeted ex vivo finger printing activity assay. 

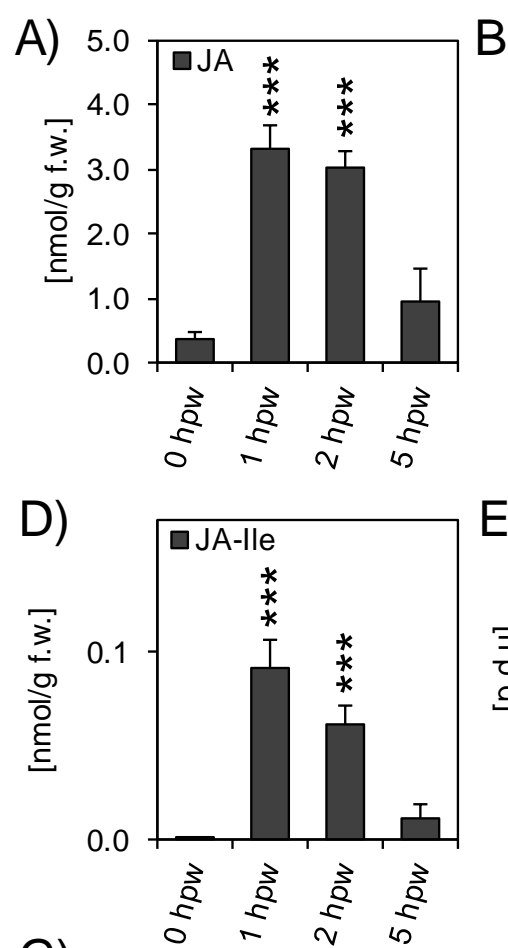

G)

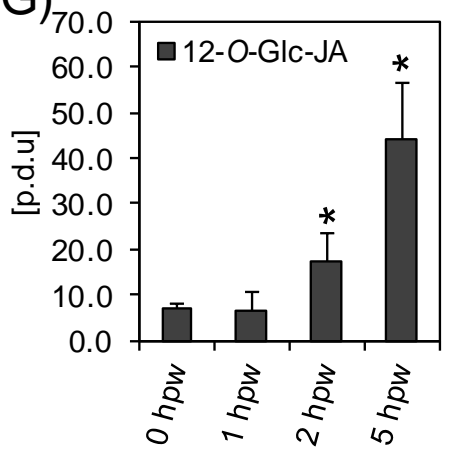

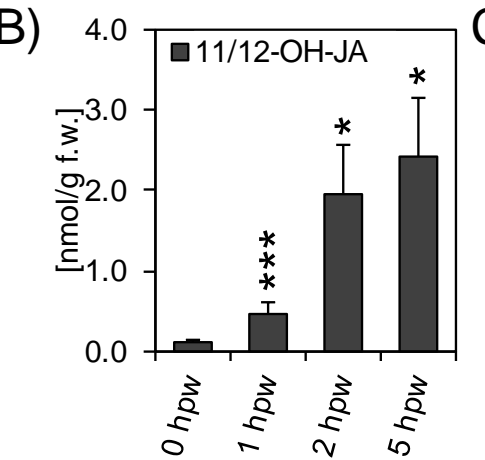

C)

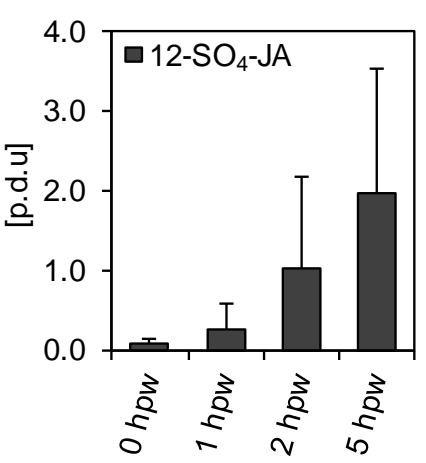

E)
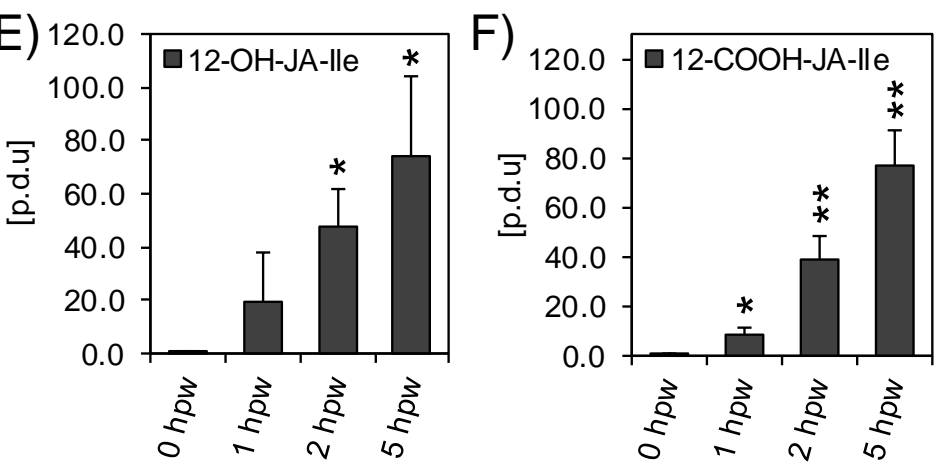

Figure 4-18: Jasmonate profiles of A. thaliana after wounding For the wounding experiment, plants were grown for six weeks at $22{ }^{\circ} \mathrm{C}$ under short day conditions ( $8 \mathrm{~h} \mathrm{light} / 16 \mathrm{~h}$ dark). Leaves were wounded three times across the mid vein by squeezing with forceps. Damaged rosette leaves were harvested at indicated time points (hours post wounding (hpw)), extracted, and analyzed by LCMS/MS. Quantitative data are given in $\mathrm{nmol} / \mathrm{g}$ fresh weight for $\mathbf{A})$ jasmonic acid (JA), B) 11/12-hydroxy-JA (11/12-OH-JA), and D) jasmonoyl-isoleucine (JA-Ile). Relative amounts were given in procedure defined units (p.d.u.) for C) 12-hydroxy-JA sulfate (12$\mathrm{SO}_{4}$-JA), E) 12-hydroxy-JA-Ile (12-OH-JA-Ile), F) 12-carboxy-JAIle (12-COOH-JA-Ile) and G) 12-O-glucosyl-JA (12-12-O-Glc-JA). Each data point represents the mean value + SD of three biological replicates from three independent experiments. 10 plants were pooled for each time point of one replicate. Asterisks indicate significance by one-sided T-Test with *p $<0.05, * * \mathrm{p}<.0 .01$, $* * * \mathrm{p}<0.005$. Samples were measured by Dr. Cornelia Herrfurth (University of Goettingen, Germany).

\subsection{GENE EDITING ENABLES INVESTIGATING UGT76E1, UGT76E2, UGT76E11, AND UGT76E12 IN A. THALIANA}

Analysis of the in vivo roles of the UGTs was initially started through transfer DNA (T-DNA) insertion lines of $U G T 76 E 1$ and $U G T 76 E 12$. However, the annotated mutants of UGT76E1 could not be confirmed in the respective locus and the homozygous mutations in the promotor and the 5 'untranslated region of UGT76E12 did not affect the abundance and distribution of jasmonates after wounding (data not shown) ( $c f$. Haroth, 2014). On top of this, the four UGT genes are located in two sets of two neighboring loci each within the A. thaliana genome, making it highly unlikely 
to obtain respective double mutants by crossing individual mutant lines (oral communication Dr. Amélie Kelly, University of Goettingen, Germany). Therefore, a different approach was initiated in this study, in which all four genes were targeted by a CRISPR/Cas9 gene editing tool to generate stable single as well as higher order mutants (Wang et al., 2015; Xing et al., 2014).

\subsubsection{THE OPTIMIZED VECTOR SYSTEM TARGETS FOUR CANDIDATES TO CAS9}

Since the here presented work deals with four candidates genes- UGT76E1, UGT76E2, UGT76E11, and UGT76E12 -, the plant CRISPR/Cas9 vector of Xing et al., 2014 was highly suitable. It was originally designed to carry four targets in separate gRNAs with individual promoters and terminators. Wang and co-workers then improved the vector system of Xing, by introducing egg-cell specific promotors and terminators (pHEE401E vector, available from Addgene, Cambridge, USA), Figure 4-19). This way, the gRNA and Cas9 are both expressed in the single-cell-state of the plant embryo and successful Cas9-activity should thus lead straight to homozygous mutations in all later plant cells. Effective and selective design of the gRNA is crucial for successful gene editing by CRISPR/Cas9. Highly specific target-sequences of UGT76E1, UGT76E2, UGT76E11, and UGT76E12 were selected based on the following criteria: Presence of a PAM, no likely off-targets, GC-content around $40 \%$, and an on-target efficiency above 0.5 (Table 4-6). These short sequences were inserted as oligonucleotides into pHEE401E for subsequent Agrobacterium-mediated transformation of A. thaliana.

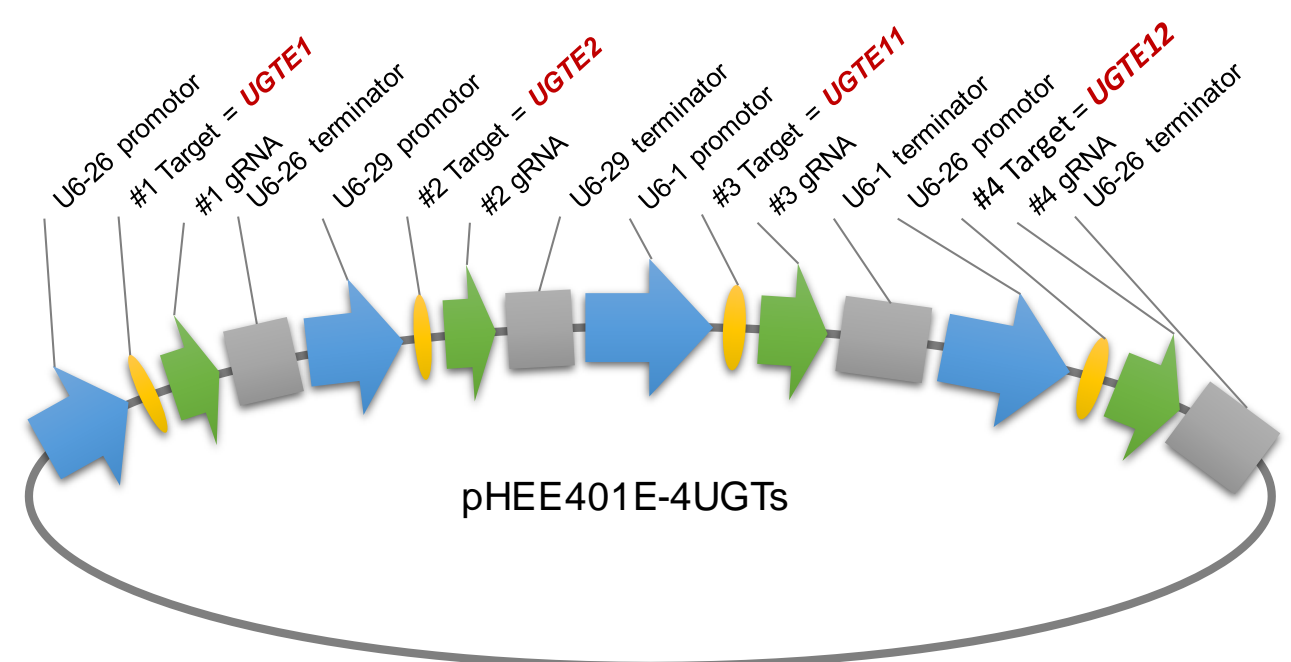

Figure 4-19: Vector maps of the CRISPR-Cas9 constructs for four UGT-target genes

A CRISPR-Cas9 approach was used to generate stable loss-of-function UGT-mutant lines of A. thaliana. The egg-cell specific promotor activates Cas9 in the one-cell phase making it possible to obtain homozygous mutants in the T1-generation already. A four-target construct was generated to target UGT76E1, UGT76E2, UGT76E11, and UGT76E12 to Cas9. The vectors are commercially available on www.addgene.org and are described in Wang et al., 2015; Xing et al., 2014. 
Table 4-6: CRISPR target sequences for UGT76E1, UGT76E2, UGT76E11, and UGT76E12

Targets were chosen with the help of three online tools: CRISPRdirect (https://crispr.dbcls.jp/), CRISPRP (http://crispr.hzau.edu.cn/CRISPR2/), and The Genetic Pertubation Platform for designing sgRNAs for CRISPRko (http://portals.broadinstitute.org/gpp/public/analysis-tools/sgrna-design). The best targets are listed which were used integrated into the CRISPR/Cas9 vector.

\begin{tabular}{|c|c|c|c|c|c|c|}
\hline Target & Sequence & Strand & Off targets & Score & $\begin{array}{l}\text { On target } \\
\text { efficiency }\end{array}$ & $\begin{array}{c}\text { GC content } \\
{[\%]}\end{array}$ \\
\hline UGT76E1 & CGGGAAGGCTCTTTACTCCAAGG & + & 0 & 99 & 0.57 & 55 \\
\hline UGT76E2 & CCCAGGCAGCTTAACTGAGTCTG & - & 0 & 95 & 0.51 & 50 \\
\hline UGT76E11 & CCATTCCAGAAAGCTTACCAGAG & - & $1(U G T 76 E 12)$ & 49 & 0.51 & 45 \\
\hline UGT76E12 & GGTGAGCTTCAAGGACTGTTTGG & + & 0 & 71 & 0.39 & 50 \\
\hline
\end{tabular}

\subsubsection{CRISPR/CAS9 INITIATES A LOSS-OF-FUNCTION MUTATION IN UGT76E1}

After plant transformation, T1 seedlings were selected by hygromycin and positive plants propagated. Interestingly, some plants had developed a phonotype that is connected to jasmonates-deficiency (Caldelari et al., 2011; Stintzi \& Browse, 2000; von Malek et al., 2002): around $5 \%$ of the transformants of the generation 1 (T1) showed an impaired silique-formation. These plants were sprayed with JA-ME after which they formed seeds in response. These T1 plants were chosen and a total of 76 plants were propagated (T2). It should be noted, that these plants did not display any growth phenotypes and that they set seeds without any application of JA-ME. Anyhow, those 76 plants were screened for alterations in their JA-catabolism as compared to wild type. To ensure seed propagation, the plants were grown under long day conditions (16 h light / $8 \mathrm{~h}$ dark) and only three leaves per plant were wounded and harvested at $2 \mathrm{hpw}$ for metabolite analyses (cf. 4.4).

To highlight metabolite differences concerning 12-OH-JA, a coefficient was calculated between the UGT-substrate, the UGT-concurrence reaction, and the UGT-product (amount of 12-OH-JA multiplied with the amount of $12-\mathrm{SO}_{4}-\mathrm{JA}$ over the amount of $12-\mathrm{O}$-Glc-JA). This coefficient was used to screen the 76 plants. Higher values depict more substrate and concurrence product than 12-O-Glc-JA. Six wild type plants show equal levels. Most of the transformed plants exhibit levels within a maximal 3-fold fluctuation. Exclusively, one plant line shows a 7-fold higher value (Figure 4-20A). For this particular plant, all four candidate loci were sequenced and compared to the wild type. The sequence alignment of $U G T 76 E 1$ revealed an insertion of a single thymidine at the expected Cas9-cutting-site (position 98 of the consensus, Figure 4-20B). This insertion causes a frame shift in the ORF of UGT76E1 and results in a stop codon shortly after (position 136 of the consensus). Therefore, it may be assumed that in this particular plant line, UGT76E1 is knocked out and not functional anymore. The sequencing was repeated three times independently supporting the mutation and confirming a homozygous ugt76el genotype.

Here, the CRISPR/Cas9 approach has successfully produced a homozygous mutation of UGT76E1 in the T2-generation. The mutation was identified by altered metabolite levels of the 
enzyme's reaction partners. This is so far the only mutation that was obtained by this approach. Obviously, more mutations of the remaining UGTE76s are needed for gaining 12-O-Glc-JAdeficient plants, which shall be screened for in the progeny of ugt76el.

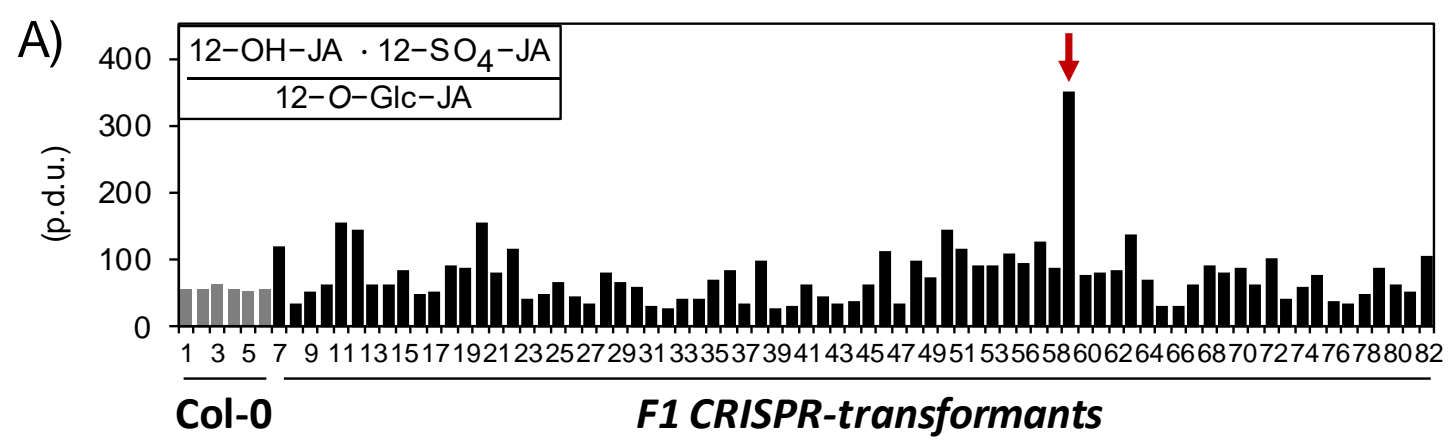

B)

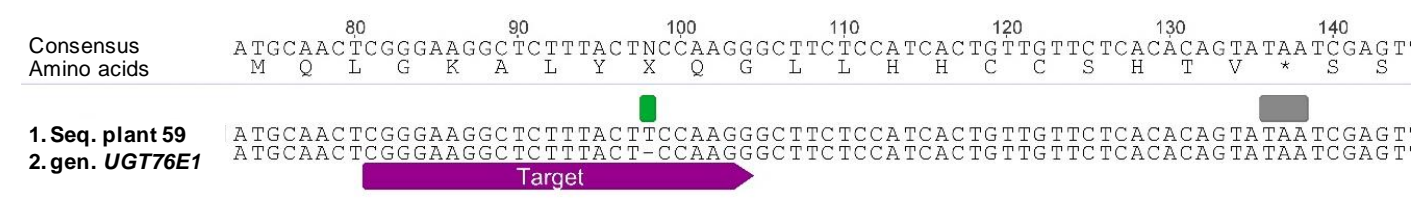

Figure 4-20: Identification of a mutation in the ORF of UGT76E1 by CRISPR-Cas9 activity

A CRISPR-Cas9 approach was used to introduce mutations in UGT76E1, UGT76E2, UGT76E11, and UGT76E12 in wild type A. thaliana (Col-0). After transformation, plant seedlings were selected by hygromycin. 76 F1-plants (7-82) were screened for alterations in the JA-catabolism in comparison to Col0 plants (1-6). Leaves were wounded three times across the mid vein by squeezing with forceps.

Damaged rosette leaves were harvested at 2 hours post wounding. After extraction, the samples were analyzed by LC-MS/MS. Special focus was given to the following metabolites: 12-hydroxy-JA (12-OHJA) as substrate of the UGT-reaction, 12-O-glucosyl-JA (12-O-Glc-JA) as its product and 12-hyroxy-JA sulfate (12-SO4-JA) as product of a concurrence reaction with 12-OH-JA as substrate. A) The product of the relative amounts of $12-\mathrm{OH}-\mathrm{JA}$ and $12-\mathrm{SO}_{4}$-JA divided by the relative amount of $12-\mathrm{O}$-Glc-JA were calculated and are shown as bar plots for all analyzed lines. Plant 59 exclusively shows the highest value (red arrow). Genomic DNA of plant 59 was sequenced. Jasmonate measurements were performed together with Dr. Krzysztof Zienkiewicz. B) Sequence alignment of the target region of plant line 59 and the corresponding UGT76E1 region in genomic DNA of Col-0. The alignment illustrates an insertion of one thymidine at the Cas9-cutting-site (green annotation). This insertion causes a stop codon in the open reading frame of the edited sequence (grey annotation) and an end of the amino acid sequence ${ }^{*}$ ). The sequencing is representative for three independent experiments. The alignment was done with the Geneious algorithm as global alignment with free end gaps and $65 \%$ cost matrix in Geneious version 8.1 (Biomatters, New Zealand). Available from http://www.geneious.com 


\section{DISCUSSION}

The role of jasmonates in plants goes far beyond plant defense towards feeding insects, and necrotrophic pathogens. Therefore, JA, JA-Ile and their derivatives may have functions in other physiological processes (Koo, 2018; Wasternack \& Hause, 2013; Wasternack \& Song, 2017). Within the catabolic fate of jasmonates, the defense-inactive 12-OH-JA has a special position because it is produced in two ways and gets modified by two reactions (see Figure 1-3) (Miersch et al., 2008). It is synthesized either by oxidation of JA by JOX1-4 (Caarls et al., 2017) or by deconjugation of the inactive 12-OH-JA-Ile by IAR3 and ILL6 (see Figure 1-3) (Widemann et al., 2015). Although both paths compete with each other (Bruckhoff et al., 2016), it was suggested that the oxidative path is favored after necrotrophic pathogen attack and the de-conjugation is preferred after wounding (Smirnova et al., 2017). The same holds true for the modifications of 12-OH-JA. The levels of 12-HSO ${ }_{4}-\mathrm{JA}$ (Gidda et al., 2003) and 12-O-Glc-JA influence each other but molecular regulations are not known yet (Mugford et al., 2009; Wasternack \& Hause, 2013). Interestingly, the enzyme(s) to specifically glycosylate 12-OH-JA were elusive although the reaction product, 12-O-Glc-JA, is known for a long time in plants (Miersch et al., 2008; Seto et $a l ., 2009)$. The glycoside might have metabolic functions in signaling, inactivation, degradation, storage, or recycling (Bowles et al., 2006; Vogt \& Jones, 2000). Thus, this work identified and characterized four UGTs with the aim to describe specific enzymes for the glycosylation of 12OH-JA to 12-O-Glc-JA. Likewise, it may be possible to complete the set of enzymes acting on jasmonates after wounding (see Figure 1-3) and to improve the knowledge of the metabolic flux around 12-OH-JA. Since 12-OH-JA may be regulated as an inactive jasmonate in regard to defense related JA-Ile responses (Miersch et al., 2008), it is not clear so far, which consequences the glycosylation or the sulfation introduced to 12-OH-JA may have (Koo, 2018; Wasternack \& Feussner, 2018). Therefore, the characterizations of the candidate UGTs were achieved by different approaches - as suggested by Vogt \& Jones, 2000 - to deal with the expected broad substrate tolerances of the enzymes and to obtain a comprehensive and conclusive picture of the catalytic activities of the JA-related UDP-dependent glycosyltransferases in A. thaliana. Furthermore, distinct biochemical data might assist evaluating complex physiological effects in vivo. 


\subsection{COMPREHENSIVE CHARACTERIZATION OF THE JA-RELATED UGTS REVEALED THEIR CATALYTIC PREFERENCES}

Briefly, UGT76E1, UGT76E2, UGT76E11, and UGT76E12 were identified as wound-induced and, therefore, JA-related $U G T$ s. Sequence-analyses identified the catalytic motifs for substrate binding at the N-terminus and UDP-Glc binding at the C-terminus. Such structural motifs are common for all UGT enzymes with the protein fold B (see Figure 1-5). There, the enzyme consists of two domains connected via a large cleft, which harbors the active center (Albesa-Jové \& Guerin, 2016; Offen et al., 2006; Wang, 2009). Logically, the substrate-binding at the N-terminal domain is less conserved and cannot be used to predict potential substrates. All four candidates belong to the UGT76 subfamily (Mackenzie et al., 1997), which is classified in the group H by sequence homology of nine conserved regions of all UGTs in A. thaliana (see Figure 4-2) (Ross et al., 2001). From the group H, three members - UGT76B1, UGT76C2, and UGT76D1 - have been characterized with catalytic activities to form HMPA-Glc, Glc-DHBA, and cytokinin glycosides, respectively (Hou et al., 2004; Huang et al., 2018; von Saint Paul et al., 2011). Thus, it was also not possible to deduce putative substrates of the candidates from these homologies. Nevertheless, UGT76B1, UGT76C2, and UGT76D1 are involved in defense responses in A. thaliana (Hou et al., 2004; Huang et al., 2018; von Saint Paul et al., 2011). In analogy to CYP94B1, CYP94B3, and CYP94C1 (see 1.1.3), which are oxidizing JA-Ile and were identified as FA-oxidases first (Benveniste et al., 2006), specific UGT may have similar catalytic tolerances. In addition to that, UGT76B1 was found to form glycosides with short OH-FA as for its published product HMPA-Glc (von Saint Paul et al., 2011). Since UGT76E1, UGT76E2, UGT76E11, and UGT76E12 are induced by wounding, they may be related to JA-metabolism and may be active on OH-FAs and 12-OH-JA. This hypothesis was supported by the activity of UGT76E1, UGT76E2, and UGT76E12 showed activity with 12-OH-JA (Table 4-4). On top of this, UGT76E1, UGT76E2, UGT76E12, and to some extent of UGT76E11 towards $\omega-\mathrm{OH}-16: 0$ and oxylipins like 11-HHT, 9-HOT, and 13-HOT (Figure 4-7, Figure 4-13). Thus, it can be concluded that UGTs active on 12-OH-JA also tolerate oxylipins due to structural similarities.

The four candidate UGTs and the control UGT, UGT74F1, specific for the glycosylation of SA (George Thompson et al., 2017; Lim et al., 2004), were characterized biochemically by different activity assays with the aim to confirm the expected activity towards 12-OH-JA, to describe the substrate preferences and, eventually, to identify new native substrates (see above) ( $c f$. Vogt \& Jones, 2000). Therefore, an activity assay with radiolabeled co-substrate (UDP- $\left.{ }^{14} \mathrm{C}(\mathrm{U})-\mathrm{Glc}\right)$, a comprehensive assay in a quasi-native substrate environment as well as detailed spectrophotometric analyses for estimating the kinetic constants were used. 
As mentioned above, UGT76E1, UGT76E2, UGT76E11, and UGT76E12 showed activity towards $\omega-\mathrm{OH}-16: 0$ and UGT76E1, UGT76E2, and UGT76E12 were also able to glycosylate 12$\mathrm{OH}-\mathrm{JA}$. For further investigations, the candidates were analyzed towards a set of putative substrates to narrow down their substrate preferences. With different sets of substrates (FAderived, plant specialized metabolites, jasmonates, and flavonoids) the specificities and necessities of the candidates towards a free or shielded carboxy group, the presence and position of a hydroxy group, and the overall size of the substrate were evaluated by an activity test with radiolabeled UDP- ${ }^{14} \mathrm{C}(\mathrm{U})$-Glc. Like this, all reactions give the same product signal and can be evaluated for one enzyme but different UGTs cannot be compared to each other. Concerning phytohormones, the UGT76E1, UGT76E2, and UGT76E12 showed product formation with 12$\mathrm{OH}-\mathrm{JA}$ exclusively. In addition, a special interest was to compare different general substrates to the preferred substrates. Lim and co-workers have tested the majority of A. thaliana UGTs towards BAs (Lim et al., 2002) and flavonoids (Lim et al., 2004). They found that a broad set of UGTs showed activity towards these general substrates. With benzoates, Lim and co-workers identified UGT74F1 to glycosylate SA to 2-O-Glc-SA preferably and the SA-GE only to a minor extent (Lim et al., 2002). This behavior could be confirmed in this study. UGT74F1 showed preference to SA and only minor activity to BA. This consistent reproduction of the activity of UGT74F1 served as positive control for the experimental setup and, hence, supports the findings for the candidates UGT76E1 and UGT76E2 as 12-OH-JA UGTs. Later Lim and co-workers found UGT76E12 and UGT74F1 to be active towards quercetin (Lim et al., 2004). The activity with flavonoids could be confirmed here for UGT76E12 but not for UGT74F1. Furthermore, the study of Lim showed minor activities of UGT76E1, UGT76E2, and UGT76E11 towards quercetin. In this study, only trace activities towards quercetin could be identified for UGT76E1, UGT76E2, and UGT76E11. Altogether, distinct specificities could be derived from OH-FAs and jasmonates as substrates: UGT76E1, UGT76E2, and UGT76E12 require - in case of fatty acids or the fatty acid derived oxylipins - a carboxy function and a hydroxy group, which is located in $\omega$-position of the carbon chain ( $\omega$-OH-16:0). In addition to this, the ex-vivo approach has shown specificity of these UGTs to oxylipins with a hydroxy group in the middle of the aliphatic chain (9-, 13HOT, 11-HHT, see Figure 4-13). In addition, small aromatic compounds and phytohormones with carboxy and/or hydroxy groups were not tolerated. Side activities towards flavonoids could only be observed with UGT76E12. Nevertheless, strong specificity UGT76E1 and UGT76E2 was found for 12-OH-JA. Jasmonates with differing oxidized groups were not preferred by UGT76E2 and not tolerated by UGT76E1. Again, a side activity of UGT76E12 could be observed with 12OH-JA but no other jasmonate.

Interestingly, a glycosylation of JA to JA-GE could not be observed in this work. JA-GE was identified as JA-derivative in N. tabacum (Swiatek et al., 2004). Although UGT74D1 was published as JGT1 (Song, 2005), this enzyme shows higher activities towards auxins as substrates 
(Jin et al., 2013; Tanaka et al., 2014). In this study, no candidate UGT tolerated the free carboxy group of JA for glycosylation. While the free carboxy group of 12-OH-JA was crucial for substrate recognition, neither UGT76E1, UGT76E2, nor UGT76E12 formed GEs with 12-OHJA as substrate (Figure 4-8). To identify an UGT with the ability to form JA-GE, it might be worth to investigate the UGTs of group L, in which most of the members form GEs (Figure 1-6). Another approach might try to identify a specific JA-GE UGT in $N$. tabacum first, because there the JA-GE has been found in response to JA-application (Swiatek et al., 2004). Also in N. tabacum, the respective UGT has still not been identified.

Besides experiments to determine the enzymatic substrate tolerances and specificities, the ex vivo metabolite fingerprinting approach was used, which brings back enzymes to their native substrate environment and challenges their selectivity. Like this, it was possible to investigate purified proteins as close as possible to their natural environment. The metabolite rich extracts of A. thaliana after wounding mimicked the natural substrate availability, what led to the identification of wound-induced oxylipins like 13-HOT as substrates of the UGTs. However, a drawback of this assay is the missing compartmentation. The plant tissue is homogenized before extraction and all metabolites are extracted equally no matter if the compounds are naturally available for the enzymes or not. Since compartmentation is very important to understand the physiological processes in vivo (Ovádi \& Saks, 2004), this has to be considered for evaluation of the ex vivo data. In this case, plant UGTs are proposed to be cytosolic enzymes (see Supplemental table 1) (Bowles et al., 2006; Lim et al., 2001; Vogt \& Jones, 2000). In line with this, oxylipins like 13-HOT, and 11-HHT might not be accessible to the UGTs because they are present in the chloroplast and only 9-HOT is cytosolic like the UGTs ( $c f$. Wasternack \& Feussner, 2018). Altogether, UGT76E1 and UGT76E2 have been characterized as 12-OH-JA UGTs. UGT76E11 showed activity towards 11-HHT, 13-HOT, and the unknown compound C11H18O3. UGT76E12 showed high activity towards the artificial $\omega-\mathrm{OH}-16: 0$, several oxylipins and to minor extent 12 OH-JA. In addition, the in vivo analyses of the candidates were started. Therefore, the CRISPR/Cas9 approach was used to knock out the candidate genes specifically and a putative loss-of-function ugt765el could be identified by an altered metabolite profile of 12-OH-JA, 12 $\mathrm{SO}_{4}-\mathrm{JA}$, and 12-O-Glc-JA.

\subsection{UGT76E1, UGT76E11, AND UGT76E12 ARE SLOW CATALYSTS}

The kinetic values of UGT76E1, UGT76E2, UGT76E11, and UGT76E12 were recorded with 9HOT and 13-HOT in continues spectrophotometric assay. In addition, UGT76E1 and UGT76E2 were measured with 12-OH-JA. As UGT76E12 has the lowest specificity towards 12-OH-JA (Table 4-4), these measurements were skipped due to substrate limitations. The experimental setup showed that the UGTs in this study were generally slow enzymes and kinetic measurements 
could just be determined with specific substrates (see Table 4-5). Noticeable, besides this study, only Jackson et al., 2001 has determined the kinetic parameters of plant UGTs with a continuous spectrophotometric assay. However, kinetic measurements are not always reproducible: the reference UGT VvGT1, which was used to determine structural motifs in UGTs (see Figure 1-5, Figure 4-3), was measured with quercetin twice before. The kinetic parameters of Ford et al., $1998\left(\mathrm{~K}_{\mathrm{M}}\right.$ of $15 \mu \mathrm{M}, \mathrm{k}_{\mathrm{cat}}$ of $19 \mathrm{mkatal} / \mathrm{kg}$ ) differ dramatically from the values of (Offen et al., 2006) ( $\mathrm{K}_{\mathrm{M}}$ of $680 \mu \mathrm{M}, \mathrm{k}_{\mathrm{cat}}$ of $0.085 \mathrm{~s}^{-1}$, appr. $\left.6.0 \mathrm{mkatal} / \mathrm{kg}\right)$. Therefore, the control enzyme UGT74F1 was used to evaluate the spectrophotometric assay and to compare the obtained kinetic values with those from the literature. In this study, the $\mathrm{K}_{\mathrm{M}}$ value for the native UGT74F1-substrate SA was $213 \mu \mathrm{M}$ and the turnover rate was $0.003 \mathrm{~s}^{-1}(0.4 \mathrm{mkatal} / \mathrm{kg})$. This $\mathrm{K}_{\mathrm{M}}$ value is very similar to that determined in a discontinuous assay by Lim and co-workers, 2002, with $230 \mu \mathrm{M}$. For the SA-UGTs of O. sativa and $N$. tabacum, much higher $\mathrm{K}_{\mathrm{M}}$ value were determined with SA, $1200 \mu \mathrm{M}$ and $900 \mu \mathrm{M}$ respectively. The turnover rate of UGT74F1 with SA is in this study is 2fold faster than the SA-UGTs of $N$. tabacum $(0.2 \mathrm{mkatal} / \mathrm{kg})$ but, at the same time, 4 -fold slower than the turnover of SA-UGTs of O. sativa (1.5 mkatal/kg, Table 4-5) (Seto et al., 2011; Seto et al., 2009). The same UGT74F1 from A. thaliana was estimated by Lim et al., 2002 as being 10fold faster $(5.8 \mathrm{mkatal} / \mathrm{kg}$ ) than here. Generally, the turnover of UGT74F1 estimated here is similar to that of UGT76E1 with 12-OH-JA from this study and UGT73B1 with flavonoids (Table 5-1). The affinity of UGT74F1 to SA is similar to many values determined for UGTs with their specific substrates (Table 5-1). In this regard, the kinetic values obtained for UGT74F1 here are comparable to those of several other kinetic studies.

The kinetic parameters of UGT76E2 with 12-OH-JA point to medium affinity and a rather fast turnover rate $\left(\mathrm{K}_{\mathrm{M}}\right.$ of $219 \mu \mathrm{M}, \mathrm{k}_{\mathrm{cat}}$ of $\left.1.4 \mathrm{~s}^{-1}\right)$; UGT76E2 was slightly less affine but 100-fold faster than UGT76E1 with the same substrate. It shows similar turnovers than the fastest plant UGTs from the comparison (Table 5-1). However Jackson et al., 2001, used a similar photometric setup and could record maximal reaction velocities, which were up to 3 -fold faster than determined for UGT76E2 in this assay (Table 5-1). Compared to the only UGTs, which have been tested with 12-OH-JA before (Seto et al., 2011; Seto et al., 2009), UGT76E2 with 12-OH-HA shows a 6-fold respective 2 -fold higher affinity and a 1500 -fold respective 58000 -fold faster turnover rate than the SA-UGT from $N$. tabacum and O. sativa. The values of UGT76E2 with 13-HOT (K $\mathrm{K}_{\mathrm{M}}$ of $4 \mathrm{mM}, \mathrm{k}_{\text {cat }}$ of $0.5 \mathrm{~s}^{-1}$ ) show also fast turnovers but unspecific affinity. This affinity is in a similar range than the affinity of the side activities of the SA-UGTs of $O$. sativa and $N$. tabacum with 12OH-JA what suggested that 13-HOT may not be the best substrate for UGT76E2 (Table 5-1). In summary, UGT76E2 seems to be a general fast UGT, but with clear affinity to its preferred substrate, 12-OH-JA.

UGT76E1 showed high affinity with 12-OH-JA $\left(\mathrm{K}_{\mathrm{M}}\right.$ of $60 \mu \mathrm{M}, \mathrm{k}_{\mathrm{cat}}$ of $\left.0.012 \mathrm{~s}^{-1}\right)$. These values are similar to the most affine measurements of comparisons and values of UGT76E12 with oxylipins 
(Table 4-5). Since UGT76E1 had to be stabilized with BSA for kinetic measurements, it is likely that the buffer conditions were not optimal for the enzyme impairing its reaction speed. Such limitations will affect the turnover but not the affinity. Certainly, UGT76E1 will show higher reaction rates in optimal conditions. However, the turnover of UGT76E1 with 12-OH-JA is still 2-fold faster than the velocity of the control UGT74F1 with its native substrate SA. Furthermore, the reaction velocity is similar to the turnovers of UGT73B1 with flavonoids and the SA-UGTs from $N$. tabacum and $O$. sativa with SA (Table 4-5). Compared to the activities of SA-UGTs from N. tabacum and O. sativa with 12-OH-JA (Seto et al., 2011; Seto et al., 2009), UGT76E1 is 22fold respectively 7 -fold more affine and 21-fold respectively 830 -fold faster than the SA-UGTs (Table 4-5). Altogether, the high affinity and the moderate turnover still give strong evidence for a natural activity of UGT76E1 towards 12-OH-JA.

Especially, the kinetic constants with oxylipin substrates were very slow. Nevertheless, the very slow turnovers of UGT76E11 and UGT76E12 with 13-HOT are similar to those of UGT73B1

Table 5-1: Comparison of kinetic parameters of UDP-dependent glycosyltransferases

UDP-dependent glycosyltransferases (UGTs) and their gene number (Arabidopsis thaliana) are listed with substrate affinity $\left(\mathrm{K}_{\mathrm{M}}\right)$ and turnover rate $\left(\mathrm{k}_{\mathrm{cat}}\right)$ of indicated substrate. The data for the first five enzymes are results of this work, while the kinetic parameters of the following UGTs are taken from the literature. 12-Hydroxy-jasmonic acid, 12-OH-JA; 13-hydroxy-octadecanoic acid, 13-HOT; salicylic acid, SA; jasmonic acid, JA; indole-3-acetic acid, IAA; indole-3-butyric acid, IBA; indole-3-proponic acid, IPA, SA-UGT from Nicotina tabacum, NtSA-UGT; SA-UGT from Oryza sativa, OsSA-UGT; glycosyltransferase 1 from Vitis vinifera, VvGT1.

\begin{tabular}{|c|c|c|c|c|c|c|}
\hline Enzyme & Gene & substrate & $\mathbf{K}_{\mathbf{M}}$ & & kcat & Reference \\
\hline & & & $(\mu \mathrm{M})$ & $\left(\mathrm{s}^{-1}\right)$ & (mkatal/kg) & \\
\hline UGT76E1 & At5g59580 & 12-OH-JA & 60 & 0.012 & 0.83 & this study \\
\hline UGT76E2 & At5g59590 & 12-OH-JA & 219 & 1.477 & 58.2 & this study \\
\hline UGT76E2 & At5g59590 & 13-HOT & 4000 & 0.500 & 18.95 & this study \\
\hline UGT76E11 & At $2 g 46670$ & 13-HOT & 156 & 0.001 & 0.18 & this study \\
\hline UGT76E12 & At $2 g 46660$ & 13-HOT & 23 & 0.001 & 0.22 & this study \\
\hline UGT76E12 & At $2 g 46660$ & 9-HOT & 22 & 0.001 & 0.33 & this study \\
\hline UGT74F1 & At $2 g 43840$ & SA & 213 & 0.003 & 0.39 & this study \\
\hline UGT72E2 & At5g66690 & Sinapic acid & 900 & - & 20.33 & Lim et al., 2001 \\
\hline UGT72E2 & At5g66690 & Sinapoyl-alcohol & 240 & - & 151.52 & Lim et al., 2001 \\
\hline UGT73B1 & At4g34138 & Quercetin & 18 & - & 0.33 & Kim et al., 2006 \\
\hline UGT73B1 & At4g34138 & Kaempferol & 26 & - & 0.25 & Kim et al., 2006 \\
\hline UGT74D1 & At $2 g 31750$ & JA & 290 & 0.035 & - & Song 2005 \\
\hline UGT74D1 & At $2 \mathrm{~g} 31750$ & IAA & 540 & 0.067 & - & Song 2005 \\
\hline UGT74F1 & At $2 g 43840$ & SA & 230 & - & 5.79 & Lim et al., 2002 \\
\hline UGT74G1-like & $N t S A-U G T$ & 12-OH-JA & 1300 & - & 0.04 & Seto et al., 2011 \\
\hline UGT74G1-like & $N t S A-U G T$ & SA & 900 & - & 0.23 & Seto et al., 2011 \\
\hline UGT74H5 & OsSA-UGT & SA & 2100 & - & 1.53 & Seto et al., 2009 \\
\hline UGT74H5 & OsSA-UGT & 12-OH-JA & 400 & - & 0.001 & Seto et al., 2009 \\
\hline UGT78A5 & $V v G T 1$ & Quercetin & 680 & 0.085 & - & Offen et al., 2006 \\
\hline UGT78A5 & $V v G T 1$ & Quercetin & 15 & - & 18.88 & Ford et al., 1998 \\
\hline UGT84A1 & At4g 15480 & Coumaric acid & 400 & - & 13.07 & Lim et al., 2001 \\
\hline UGT84A1 & At4g 15480 & Sinapic acid & 580 & - & 6.39 & Lim et al., 2001 \\
\hline UGT84B1 & At2g23260 & IAA & 240 & - & 180 & Jackson et al., 2001 \\
\hline UGT84B1 & At $2 g 23260$ & IBA & 150 & - & 122 & Jackson et al., 2001 \\
\hline UGT84B1 & At $2 g 23260$ & IPA & 140 & - & 113 & Jackson et al., 2001 \\
\hline
\end{tabular}


with quercetin and kaempferol (Kim et al., 2006). Like for UGT76E1, the slow reaction velocities are thought to be influenced by the buffer conditions. Hence, for UGT76E11 and UGT76E12 with 13-HOT, the focus was given to the affinities $\left(\mathrm{K}_{\mathrm{M}}\right)$ and not to the reaction velocities $\left(\mathrm{k}_{\mathrm{cat}}\right)$. The affinities of UGT76E12 with 9-HOT and 13-HOT are the highest, which were determined in this work, and similar to the $\mathrm{K}_{\mathrm{M}}$ values of UGT76E1 with 12-OH-JA, and VvGT1 and UGT73B1 with quercetin (Table 4-5). Since these affinities were identified to be the native activities of the respective enzymes, it is very likely that UGT76E12 might be involved in the glycosylation of oxylipins and especially 13-HOT as well. UGT76E11 shows similar behavior with 13-HOT. The affinity $(156 \mu \mathrm{M})$ is not as high as for UGT76E12 but still comparable to $\mathrm{K}_{\mathrm{M}}$ values of natural reaction partners like UGT74F1 with SA, UGT84B1 with auxins, and UGT72E2 with sinapoylalcohol (Table 4-5). However, UGT76E11 showed also activity against 11-HHT and C11H18O3, and it has been shown to form flavonoid-glycosides (Li et al., 2018). Those activities have to be considered for a complete characterization of UGT76E11 with detailed kinetic measurements.

In summary, the kinetic data support the high affinity of UGT76E12 towards oxylipins and a potential role in glycosylation of these plant metabolites. Further, the kinetic parameters reveal high affinity but low turnover of UGT76E1 towards 12-OH-JA and fast turnover of UGT76E2 with 12-OH-JA but with lesser affinity. These catalytic differences are likely to influence the physiological function of UGT76E1 and UGT76E2 in plantae.

\subsection{UGT76E1 GLYCOSYLATES 12-OH-JA WITH HIGH SPECIFICITY}

In the beginning, it was postulated that the jasmonate metabolism might be driven by chronological protein biosynthesis of the responsible proteins (see 2). This hypothesis led to the identification of UGT76E1 by co-regulation with JOX3 (Table 4-1). The substrate for glycosylation - 12-OH-JA - shows elevated levels from 1 hpw on (see 4.4) and might trigger 12 $O$-Glc-JA synthesis. This matches with the highest expression of UGT76E1 in the quantitative expression data as well as the transcriptomic data with accumulations at $2 \mathrm{hpw}$ in a JA-dependent manner (see 4.1.2, 4.1.3). On top of this, UGT76E1 is co-regulated with genes involved in both synthetic paths of 12-OH-JA (JOX3 and ILL6, Table 4-2). Hence, the expression pattern of $U G T 76 E 1$ is temporally connected with reactions producing its substrate and specifically classified into the wound-induced defense pathway in A. thaliana (Supplemental figure 4A).

UGT76E1 showed problems in protein purification and protein stability. In contrast to its closest homolog - UGT76E2 (88\% identity) -, the purification of UGT76E1 has needed several optimizations (see Figure 4-5) and yielded the lowest amounts of all purifications. However, this is in analogy with (Vogt \& Jones, 2000), who characterize UGTs as generally labile enzymes. Furthermore, Bowles and co-authors suggest that in vitro experiments with UGTs are difficult because specific co-factors, protein-protein interactions and metabolic channeling may be missing 
in in vitro reactions. Thus, the protein stability of UGT76E1 might be caused by the lack of cofactors, assisting or stabilizing proteins. At least in vitro, the enzyme might need special conditions regarding the buffer composition as well as additives to stabilize the protein. During kinetic measurements, UGT76E1 had to be stabilized with $0.13 \%$ BSA and still showed slow reaction rates with 12-OH-JA. Due to this low turnover, UGT76E1 showed 30-fold lower catalytic efficiency than UGT76E2 although UGT76E1 had high affinity to 12-OH-JA ( $\mathrm{K}_{\mathrm{M}}$ of $\left.60 \mu \mathrm{M}\right)$. However, it could be shown by different enzymatic assays (see Figure 4-10, Figure 4-13, Table $4-5$ ) that the glycosylation of $12-\mathrm{OH}-\mathrm{JA}$ to $12-\mathrm{O}$-Glc-JA is the preferred reaction of UGT76E1 in vitro. Likewise, different functional properties of the substrate could be identified as being crucial for catalysis: UGT76E1 seems to require a hydroxyl group and carboxy function. Though the hydroxy group is glycosylated (Figure 4-8), a free carboxy group is required for catalysis. The fatty alcohol (OH-C16) and 12-OH-JA-derivatives with a shielded carboxy group, like 12-OHJA-ME (tested in the radiolabeled assay) and the 12-OH-JA-Ile (provided in the ex vivo extracts), were not used as substrates by UGT76E1.

Furthermore, UGT76E1 was the only candidate, for which the in vivo analysis could be started in this study. A recently described CRISPR/Cas9 toolbox (Wang et al., 2015; Xing et al., 2014) was used to target all four candidates to Cas9 endonuclease activity. As a result, a putative loss-offunction ugt76el single mutant plant could be identified (Figure 4-20B). The specific mutant line was found by an altered distribution of the substrate, concurrence product, and product (12-OHJA * 12-HSO4-JA / 12-O-Glc-JA, Figure 4-20A). Surprisingly, the abundance of 12-O-Glc-JA itself was not reduced in this mutant line (data not shown). Thus, it is likely that UGT76E1, UGT76E2, and UGT76E12 may act redundantly. UGT76E1 and UGT76E2 both were characterized to specifically glycosylate 12-OH-JA in vitro and, in addition, UGT76E12 showed minor activity against 12-OH-JA (Table 4-4). In this respect, UGT76E1 and UGT76E12 were both significantly induced after wounding (Figure 4-1). Additionally, also UGT76E2 might compensate for reduced 12-OH-JA-glycosylation in vivo although it illustrated a different expression pattern (see Figure 4-1, Supplemental figure 3, Supplemental figure 4). To generate a 12-O-Glc-JA-deficient mutant line at least these three UGTs active on 12-OH-JA have to be mutated.

In summary, this study was aware that UGTs tend to be rather unspecific enzymes, which have to be tested with different substrates to identify their native reactions (Bowles et al., 2006; Vogt $\&$ Jones, 2000). Thus, the clear preference of UGT76E1 to 12-OH-JA in different activity assays emphasizes a potential role of UGT76E1 as natural 12-OH-JA-UGT in A. thaliana (Figure 5-1). 


\subsection{UGT76E2 FORMS 12-O-GLC-JA WITH HIGH SPECIFICITY AND TURNOVER NUMBERS}

The transcript of the next candidate, UGT76E2, did not show significant accumulation after wounding in the leaves of A. thaliana, which were analyzed by RT-PCR (see Figure 4-1). Nevertheless, UGT76E2 was identified by co-expression with $S T 2 a$ and shows a similar expression profile as $S T 2 a$ (data not shown, eFP-Browser access 02.05.2018). This shared profile depicted its induction after biotic and abiotic stresses whereas UGT76E1 showed induction after wounding exclusively. I(Supplemental figure 4B) As mentioned before, 12-OH-JA does not inhibit root growth like JA and JA-Ile. However 12-OH-JA and 12-O-Glc-JA were identified as tuber-inducing compounds in S. tuberosum (Yoshihara et al., 2014). Therefore, 12-OH-JA and 12-O-Glc-JA may have a physiological role in below-ground parts of plants, at least in growthregulation of roots. The constitutive expression of UGT76E2 may be connected to this function but localization and expression studies of UGT76E2 have to be done to reveal the presence and a putative function of UGT76E2 roots.

The enzyme showed generally high activities in all experiments and it was the enzyme with the best turnover rate $\left(1.4 \mathrm{~s}^{-1}\right)$ and the highest catalytic efficiency $\left(67.44 * 10^{-4} \mathrm{~s}^{-1} \mu \mathrm{M}^{-1}\right)$ of the four candidates (Table 4-5). UGT76E2 is about 100-times faster but also slightly less affine for 12OH-JA than UGT76E1. Its kinetic constants with 12-OH-JA are $\mathrm{K}_{\mathrm{M}}$ of $219 \mu \mathrm{M}$ and a maximal turnover of $1.4 \mathrm{~s}^{-1}$. Surprisingly, the turnover of UGT76E2 with 13-HOT was also fast with $0.5 \mathrm{~s}^{-}$ ${ }^{1}$ though the catalytic affinity was very low and the $\mathrm{K}_{\mathrm{M}}$ could just be estimated with $4 \mathrm{mM}$. Hence, UGT76E2 is assumed to have a clear preference towards its native substrate, 12-OH-JA (Figure 5-1). Similar to UGT76E1, certain structural requirements can be assumed for the substrates of UGT76E2: a carboxy function and a hydroxy group of the substrate is required. However, the position of the hydroxy group is less important: UGT76E2 shows minor activities towards hydroxy groups close to the carboxy group (3-OH-16:0, Figure 4-10), similar activities than UGT76E12 towards hydroxy groups in the middle of the unsaturated aliphatic chain (11-HHT and 13-HOT, Figure 4-13), and strong activities towards $\omega$-hydroxy groups ( $\omega$-OH-16:0, 12-OHJA, Figure 4-10). In addition to this, UGT76E2 was the only UGT that tolerates a shielded carboxy group to minor extent (12-OH-JA-ME, Figure 4-10) but bigger groups attached inhibited catalysis (12-OH-JA-Ile, Supplemental figure 14). Hence, the specificity of UGT76E2 is not as tight as for UGT76E1 but shows strong preference for 12-OH-JA (Figure 5-1). The reason for this may be its higher activity. 


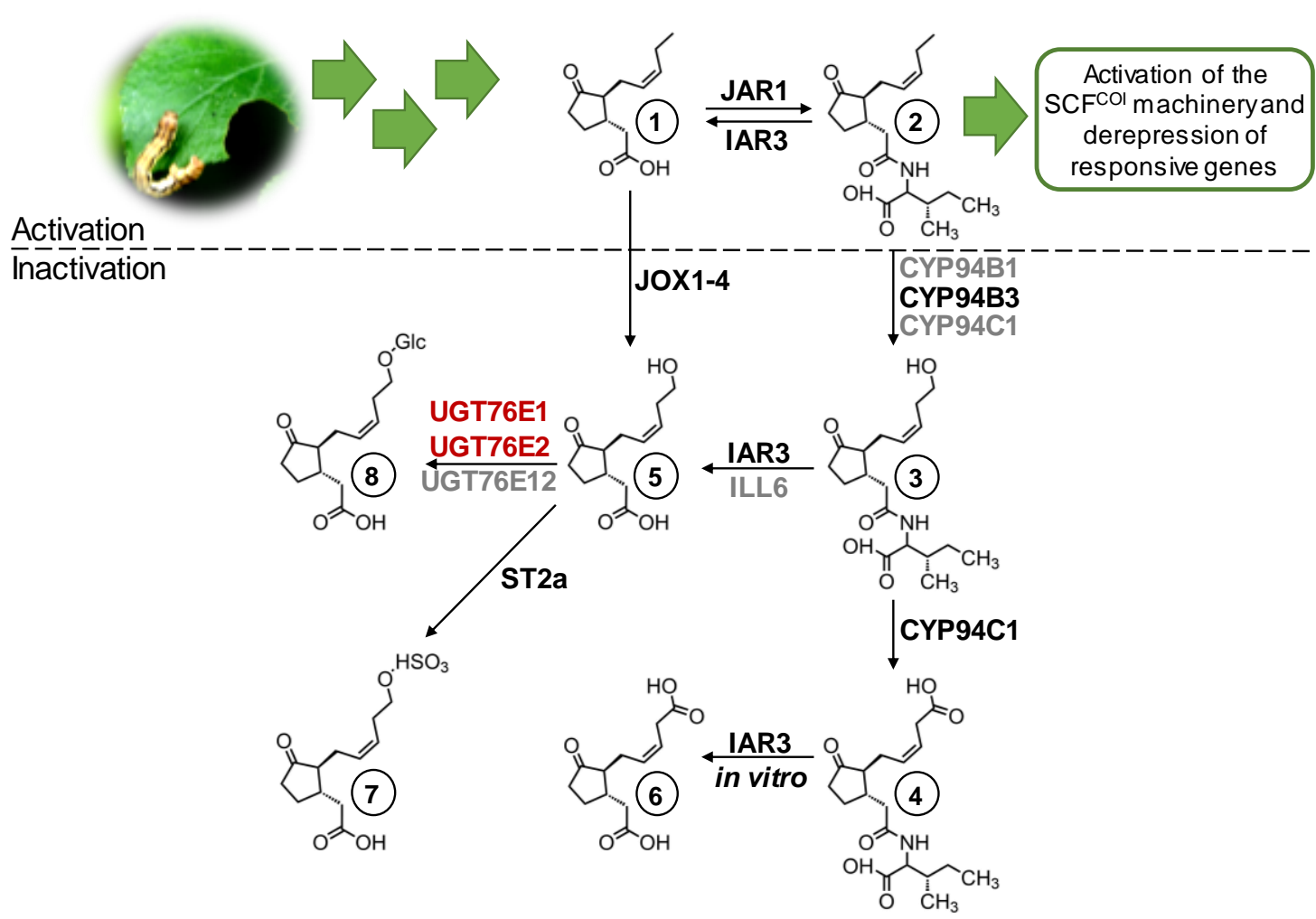

Figure 5-1: UGT76E1, UGT76E2 complete the set of jasmonate-enzymes after wounding

Wound stimuli like feeding insects lead to the activation of jasmonic acid (JA, 1) biosynthesis. JA gets active as hormone by the conjugation to isoleucine. Jasmonoyl-isoleucine (JA-Ile, 2) stimulates derepression of JA-responsive genes via proteasomal degradation. CYP94B1, CYP94B3 and CYP94C1 oxidize JA-Ile to 12-hydroxy-JA-Ile (12-OH-JA-Ile, 3) and CYP94C1 to 12-carboxy-JA-Ile (12-COOHJA-Ile, 4). JA-Ile, 12-OH-JA-Ile, and 12-COOH-JA-Ile get de-conjugated by IAA-alanine-resistant 3 (IAR3) and IAA-leucine-resistant-like 6 (ILL6) forming JA, 12-hydroxy-JA (12-OH-JA, 5) and 12carboxy-JA (12-COOH-JA, 6), respectively. JA is oxidized to 12-OH-JA by jasmonate-induced oxidases 1 to 4 (JOX1-4). 12-OH-JA gets modified to 12-OH-JA-sulfate (12-HSO -JA, 7) by sulfotransferase 2a (ST2a). This work identified two specific UDP-dependent glycosyltransferases (UGTs, highlighted in red), which glycosylate 12 -OH-JA to $12-O$-glucosyl-JA (12-O-Glc-JA, 8). A third UGTs showed minor activity forming $12-O$-Glc-JA. Scheme modified from Wasternack \& Hause, 2013, additions from Caarls et al., 2017; Kitaoka et al., 2014; Widemann et al., 2016; Zhang et al., 2016. Photo was taken from Iven et al., 2014.

\subsection{UGT76E11 SHOWS ACTIVITIES TOWARDS OXYLIPINS AND A SO FAR UNKNOWN METABOLITE}

Next, UGT76E11 showed high expression in A. thaliana after wounding in a JA-related manner (Figure 4-1, Supplemental figure 2, Supplemental figure 6). Additionally, the transcription of UGT76E11 displayed constitutive and high expression after different abiotic stresses Supplemental figure 5. In another study, UGT76E11 was found to be induced after salinity, drought and hydrogen peroxide treatment in A. thaliana (Li et al., 2018). Such an expression profile of UGT76E11 may be not suitable for specific glycosylation of jasmonates after wounding. 
UGT76E11 illustrates overall non-specific and low signals in the radiolabeled substrate assay. In this case, the set of substrates in this experiment was most likely not suitable to identify specific substrates of UGT76E11. The work of Li and co-workers, 2017, has identified flavonoids, like quercetin, kaempferol, and naringenin to be the preferred substrates of UGT76E11 in vitro and in vivo (Li et al., 2018). However, this behavior could not be confirmed here, the radiolabeled substrate assay as well as the ex vivo approach did not identify activities towards quercetin, dhkaempferol or other flavonoids (Figure 4-10,4.3.8). On the other side, the ex vivo analysis could identify specific activities of UGT76E11 from the metabolite-enriched extracts: 11-HHT, 13HOT, and a native compound of the elemental composition of $\mathrm{C} 11 \mathrm{H} 18 \mathrm{O} 3$. This unknown metabolite neither gave any databank hit nor could be structurally identified by MS/MS fragmentation analysis. From the overall ex vivo data set, it could be concluded that $\mathrm{C} 11 \mathrm{H} 18 \mathrm{O} 3$ is not wound-induced but rather constitutively present in all samples. This is consistent with a more constitutive expression profile of UGT76E11. An identity of the metabolite might enlighten the specific and high activity of UGT76E11 towards this compound. The activity towards 11HHT and 13-HOT was similar for all four tested UGTs of the 76E-subfamily and might be due to their protein homology. Nevertheless, it may be important to test the different activities of UGT76E11 from this study (11-HHT, 13-HOT, C11H18O3) with the findings of Li and coworkers (flavonoids). There, they tested specifically and exclusively flavonoids with UGT76E11. Therefore, it may be interesting to compare the activities towards oxylipins with the published activities towards flavonoids determine the best of them.

\subsection{UGT76E12 PREFERS OTHER OXYLIPINS OVER 12-OH-JA}

UGT76E12 could be identified as well by transcript accumulation after wounding in a JAdependent manner (see 4.1.2). The TAIR eFP Browser shows for UGT76E12 strong induction in response to wounding and to osmotic stress. UGT76E12 has been found as stress-induced UGT before (Rehman et al., 2018; von Saint Paul et al., 2011). In a general ranking after biotic and abiotic stress, UGT76E12 was on the fifth place of all induced UGTs in A. thaliana. Previous work only investigated the top hit, which was UGT76B1 that forms HMPA-Glc (von Saint Paul et al., 2011). However, this supports the more general stress-related expression of UGT76E12. In another study, UGT76E12 was found to be up-regulated in ABA-sensitive seedlings and after 12OPDA-application (Rehman et al., 2018). The induction by the JA-precursor 12-OPDA might explain the JA-dependency in this work. However, dissecting responses after 12-OPDA- or JAapplication needs additional experiments, because 12-OPDA will be converted in planta into JA. In comparison with UGT76E1, UGT76E2 and UGT76E11, UGT76E12 shows the highest rates of product formation with $\omega-\mathrm{OH}-16: 0$ and oxylipins like 11-HHT, 9-HOT and 13-HOT. In addition, UGT76E12 also accepts 12-OH-JA as substrate but to a lower extent (see Figure 4-13, 
Table 4-4). Here again, a free carboxy group together with the hydroxy group are necessary for catalysis because JA, 12-OH-JA-ME, 16:0 and the fatty alcohol OH-C16 were no substrates for UGT76E12. In respect to the position of the functional group, UGT76E12 seems to be more specific than UGT76E2. It tolerates the free hydroxy group at the $\omega$-position ( $\omega$-OH-16:0, see Figure 4-10) and in the middle of the molecule (11-HHT, 9-HOT, and 13-HOT, see Figure 4-13 and Table 4-4) but not in the direct neighborhood of the carboxy terminus in $\alpha$ - or $\beta$-position (see Figure 4-10). However, the kinetic parameters could be measured with 9-HOT ( $\mathrm{K}_{\mathrm{M}}$ of $22 \mu \mathrm{M}$ ) and 13-HOT ( $\mathrm{K}_{\mathrm{M}}$ of $\left.23 \mu \mathrm{M}\right)$. These are the best substrate affinities, estimated for all analyzed candidates with their preferred substrates. The turnover rates are very low in comparison to the other UGTs tested (see Table 5-1). Surprisingly, the targeted activity assays based on LC-MS measurements showed a high relative product formation for $13-O-G l c-H O T$ and $9-O-G l c-H O T$ (see Table 4-4). The glycosylation of 9-HOT and 13-HOT by UGT76E12 is a very interesting finding (Figure 5-2). It is the first time that UGTs have been described to be specific to these oxylipins. Since oxylipins are FA-derivatives, glycosylation might increase their solubility in the plant cell. Additionally, glycosylated oxylipins have been found in L. paucicostata (Kai et al., 2010) but not in A. thaliana so far. Because different oxylipins may have different functions in plants (see 1.1.1), UGT76E12 might be involved in different aspects of oxylipin regulation or even signaling. For example, pool of green leaf volatiles may be influenced (Matsui, 2006) or the oxylipin-driven defense against pathogens in N. tabacum may be regulated (Mene-Saffrane et al., 2003) with activity against 9-HOT. As 13-HOT is produced by the 13-LOX-pathway, an early interference with the pathway and its by-products is possible by storing glycosylated oxylipins in the plants vacuole after wounding. In this case, 13-HOT accumulates after SA-treatment and 13HOT alone is sufficient to induce pathogenesis-related genes in Hordeum vulgare (Weichert et al., 1999). Altogether, UGT76E12 shows high preference towards the oxylipin substrates and there are different putative functions of oxylipin-glycosides but all these hypotheses have to be evaluated in future work. 


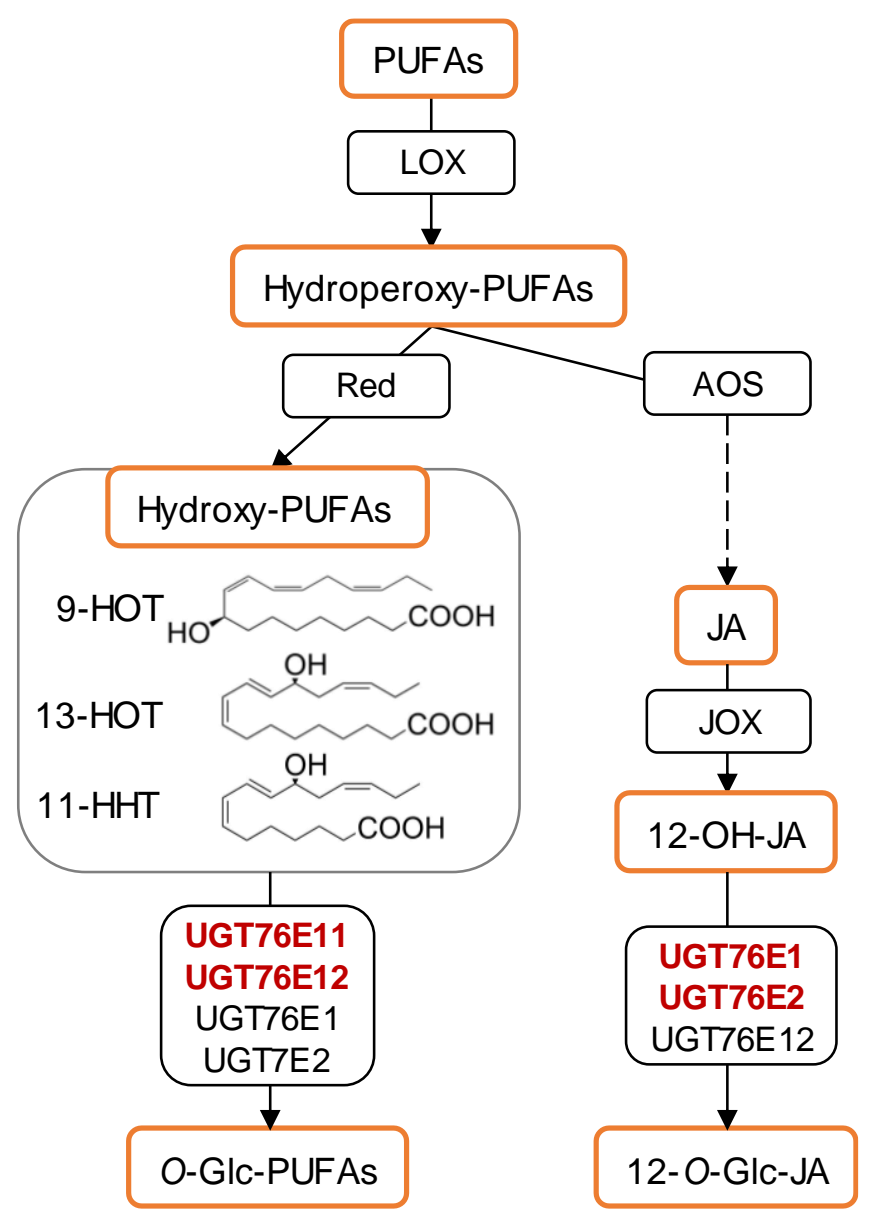

Figure 5-2: The candidate UGTs glycosylate oxylipins

Lipoxygenases (LOXs) oxygenate polyunsaturated fatty acids (PUFAs) to hydroperoxy-PUFAs. Those molecules can be metabolized to diverse oxylipins. In particular, reductases (Red) produce hydroxy-PUFAs like 9-hydroxyoctadecatrienoic acid (9-HOT), 13hydroxy-octadecatrienoic acid (13-HOT), and 11-hydroxy-hexadecatrienoic acid (11-HHT). In parallel, an allene oxide synthase (AOS) and others initiate synthesis of jasmonic acid (JA). JA may be oxidized to 12-hydroxy-JA (12-OHJA) by jasmonate-induced oxidases (JOX). Both, hydroxy-PUFAs and 12-OHJA, can be glycosylated by the candidate UDP-dependent glycosyltransferases (UGTs), UGT71E1, UGT76E2, UGT76E11, and UGT76E12. These produce $12-O$-glucosyl-JA (12-O-Glc-JA) and $O$-glucosyl-PUFAs ( $O$-Glc-PUFAs). $O$-Glc-PUFAs have been described in Lemna paucicostata (Kai et al., 2010), but no function has been assigned yet. Figure was modified from Wasternack \& Feussner, 2018.

Additionally, UGT76E12 glycosylated flavonoids like quercetin (appr. $33 \%$ of $\omega-\mathrm{OH}-16: 0$ ) and dh-kaempferol (appr. $66 \%$ of $\omega-\mathrm{OH}-16: 0$, see Figure 4-10). This activity has been described before (Lim et al., 2004). In that work, UGT76E12 showed high activity towards quercetin and Lim and co-workers speculated that UGT76E12 may be suitable for industrial application and producing high-value natural glycosides. This behavior could be confirmed in this work although the activity towards flavonoids was not the preferred activity of UGT76E12. Furthermore, UGT76E12 showed minor activity towards 12-O-Glc-JA. Between the candidates, UGT76E12 has produced similar amounts of 12-O-Glc-JA than UGT76E1 (see Figure 4-10, Table 4-4). However, the catalytic efficiency of UGT76E1 with 12-OH-JA $\left(2.02 * 10^{-4} \mathrm{~s}^{-1} \mu \mathrm{M}^{-1}\right)$ was 10 -fold higher than the efficiency of UGT76E12 with its preferred substrates 9-HOT $\left(0.33 * 10^{-4} \mathrm{~s}^{-1} \mu \mathrm{M}^{-}\right.$ $\left.{ }^{1}\right)$ and 13-HOT $\left(0.22 * 10^{-4} \mathrm{~s}^{-1} \mu \mathrm{M}^{-1}\right.$, see Table 4-5). Similar to other studies of different UGTs showing side activities with 12-OH-JA (Seto et al., 2011; Seto et al., 2009), the activity of UG76E12 might be similar due to structural similarities of 12-OH-JA to $\omega-\mathrm{OH}-16: 0$ and 13-HOT.

\subsection{EVIDENCE FOR PHYSIOLOGICAL RELEVANCE OF 12-O-GLC-JA}

The metabolite 12-O-Glc-JA has been found in different plants (Miersch et al., 2008; Seto et al., 2009). However, the responsible enzymes have been elusive until now. UGT76E1 and UGT76E2 
have shown their preference to catalyze the glycosylation of 12-OH-JA. From here, the logical step is to investigate glycosylation in vivo. This was started by a CRISPR-Cas9 approach, which could generate at least a ugt76el single mutant until now. This single mutation was already sufficient to alter the abundance of metabolites, which are connected to 12-OH-JA (Figure 4-20). However, the levels of $12-O$-Glc-JA alone were not reduced (data not shown). Therefore, generation of double (ugt76e1/ugt76e2) or triple (ugt76e1/ugt76e2/ ugt76e12) mutants of the UGTs might elucidate the role of 12-O-Glc-JA further. Thus, the CRISPR/Cas9 approach has to be repeated in homozygous ugt76el-background to obtain double or multiple mutants of the UGTs. This might enlighten the physiological role of $12-O-G l c-J A$ and the respective enzymes. Deletion of all UGTs responsible for 12-O-Glc-JA synthesis is the first possibility to investigate the role of $12-O-G l c-J A$ independently of $12-\mathrm{OH}-\mathrm{JA}$ and vice versa. So far, it was not possible to assign functions of 12-OH-JA and its glycoside individually. Both seem to induce tuber formation in S. tuberosum (Yoshihara et al., 2014), both function as COI/JAZ-independent leaf closing factor in S. samen (Nakamura et al., 2011), and both might be mobile metabolites in A. thaliana (Seto et al., 2009). In this regard, it is important to specifically assign the observations to one metabolite and elucidate the function of jasmonates next to wounding.

However, the existing knowledge provides more evidence of a metabolic function of $12-O$-GlcJA than for its concurrence product $12-\mathrm{HSO}_{4}-\mathrm{JA}$. In respect to a re-localization upon glycosylation, it remains to be clarified if $12-O$-Glc-JA is transported into the vacuole like for 2$O$-Glc-SA (Dean et al., 2005; Dean et al., 2003) or into the apoplast. An export of glycosylated phytohormones into the apoplast has only been shown for ABA (Dietz et al., 2000). However, JAT1, which seem to transport JA and JA-Ile trough the plasma membrane, may have the potential to transport jasmonates like $12-\mathrm{O}$-Glc-JA as well. Preliminary analysis of petiole exudates of wounded A. thaliana may hint towards elevated levels of $12-O$-Glc-JA and other glycosylated plant hormones in the apoplast (data not shown). In addition, a specific hydrolase enzyme in O. sativa strengthen a reversible storage function at least in rice (Wakuta et al., 2010).

As described, 12-OH-JA is modified in two reactions, which results in 12-O-Glc-JA and12- $\mathrm{HSO}_{4}$ JA. Concerning this, it is tempting to speculate why two metabolic modifications are needed. One modification may therefore be necessary to bypass the function of the other (oral communication Prof. Dr. R. Solano, Spanish National Research Council, Madrid, Spain). In this regard, 12-OGlc-JA is more likely to have relevance in plantae than its concurrence product $12-\mathrm{HSO}_{4}-\mathrm{JA}$, for which no function has been assigned until now (see 1.1.3) (Gidda et al., 2003; Koo, 2018). On top of this, UGT76E1 and UGT76E2 are characterized to specifically form 12-O-Glc-JA while being differentially expressed in roots and shoots. This might support a distinct physiological relevance of $12-O$-Glc-JA in different tissues and processes. 


\subsubsection{2-O-GLC-JA-ILE CANNOT BE IDENTIFIED IN $A$. THALIANA}

So far, 12-O-Glc-JA-Ile has been found once in plants by (Kitaoka et al., 2014). In that work, they identified the compound by targeted LC-MS/MS analysis of wounded plant material related to an authentic chemical standard. Therewith, Kitaoka and co-workers were able to detect low amounts of $12-O$-Glc-JA-Ile at $6 \mathrm{hpw}$ in three plant species: A. thaliana $(11 \pm 5 \mathrm{pmol} / \mathrm{g} \mathrm{f.w}$. $)$, N. tabaccum ( $23 \pm 6 \mathrm{pmol} / \mathrm{g}$ f. w.), and G. $\max (22 \pm 5 \mathrm{pmol} / \mathrm{g}$ f. w.) (Kitaoka et al., 2014). Nevertheless, these abundances have big deviations and those signals might be at the detection limit of that method since a robust signal of e.g. 12-OH-JA-Ile are three orders of magnitude higher $(9813 \pm 2473 \mathrm{pmol} / \mathrm{g} \mathrm{f}$. w). 12-OH-JA-Ile was also measured in this study. Although, no quantitative levels could be determined, the 12-OH-JA-Ile accumulated to significant levels after wounding. 12-OH-JA-Ile was related to an internal JA-Ile-standard and shows much higher relative levels similar to the abundance in Kitaoka et al., 2014. However, IAR3 accepts 12-OGlc-JA-Ile for hydrolytic cleavage to 12-O-Glc-JA in vitro (Kitaoka et al., 2014), which may support a physiological existence of $12-O$-Glc-JA-Ile.

This work showed significant accumulation of 12-OH-JA-Ile after wounding (see Figure 4-18E). Therefore, the potential precursor-molecule was present in $A$. thaliana but the glycoside could not be identified in this study. Since no chemical standard was available here, 12-O-Glc-JA-Ile was searched by exact mass in the LC-MS analyses of the ex vivo approach with and without of enzymes (Supplemental figure 14). The samples were investigated in both ionization modes with two independent LC-HR-MS-methods. 12-O-Glc-JA-Ile could neither be detected as internal metabolite of A. thaliana in the enriched metabolite extract $5 \mathrm{hpw}$ nor as product of UGT76E1, UGT76E2, UGT76E11 or UGT76E12 with the native substrate 12-OH-JA-Ile (Supplemental figure 14). Anyhow, it might be possible that so far unknown UGT(s) are responsible for the glycosylation of 12-OH-JA-Ile although the co-expression analysis performed in this work did not give any hit for putative candidates around 12-OH-JA-Ile (see 4.1.1).

Altogether, this study was not able to detect $12-O$-Glc-JA-Ile in A. thaliana after wounding. However, it is concluded that none of the investigated UGTs has the potential to use 12-OH-JAIle as substrate. Kitaoka et al., 2014 is still the exclusive recourse of the abundance and future work has to challenge this again with optimized detection methods to prove the physiological presents and relevance of $12-O-G l c-J A-I l e$.

\subsubsection{2-COOH-JA WAS IDENTIFIED IN WOUNDED LEAVES OF A. THALIANA}

Up to now, the new metabolite 12-COOH-JA has been identified in flowers of A. thaliana (Bruckhoff et al., 2016). In addition, 12-COOH-JA and 12-OH-JA-Ile accumulated in a jox2 mutant without wounding resulting in an elevated defense responses (Smirnova et al., 2017). 
Here, the compound was identified as wound-induced metabolite in A. thaliana leaves in the extracts of the ex vivo metabolite fingerprinting experiment (see 4.3.6). This finding supports the mutant analysis of Smirnova and co-workers, 2016, and puts 12-COOH-JA not only in the context of flower development but also in the wound response of A. thaliana. Nevertheless, it is not clear how the molecule is formed. Most likely, 12-COOH-JA is produced by hydrolytic cleavage of 12-COOH-JA-Ile by IAR3. This is supported by different approaches: the accumulation of 12 COOH-JA is dependent of CYP94C1 (Bruckhoff et al., 2016), it is produced by de-conjugation of 12-COOH-JA-Ile by IAR3 in vitro (Zhang et al., 2016), and its abundance is strongly related to 12-COOH-JA-Ile levels (Smirnova et al., 2017; Widemann et al., 2016). This hypothesis has to be challenged in future experiments with detailed phytohormone analysis of wounded A. thaliana in wild type, the cyp $94 c 1$ and the iar3 mutants of A. thaliana. The wound-induction of 12-COOH-JA gives new insights in the complex metabolic fate of jasmonates and offers a new approach to study a putative metabolic function of 12-COOH-JA. 


\section{OUTLOOK}

This study provided detailed biochemical characterizations of UGT76E1, UGT76E2, UGT76E11, and UGT76E12. Thereby, UGT76E1 and UGT76E2 could be identified as specific 12-OH-JAUGTs in vitro. UGT76E12 showed minor activity to 12-OH-JA. Its preferred substrates are oxylipins.

The next step in the project has to be the physiological investigation of the UGTs. It started by now with the generation of a ugt76e1 single mutant by a CRISPR-Cas9 approach. The metabolite analysis showed that the 12-O-Glc-JA-content in planta was not reduced. However, the ratio of metabolites, which are connected to 12-OH-JA, was altered (Figure 4-20). Therefore, it is important to generate also double (ugt76e1/ugt76e2) or triple (ugt76e1/ugt76e2/ ugt76e12) mutants of the UGTs to deplete 12-O-Glc-JA in vivo. Thereby, it might be possible to investigate the role of $12-O-G l c-J A$ independently of $12-\mathrm{OH}-\mathrm{JA}$ and vice versa in A. thaliana. Still, the CRISPR/Cas9 approach is favored to obtain such double or multiple mutants, because UGT76E1 and UGT76E2 as well as UGT76E11 and UGT76E12 are located as direct neighbors on the A. thaliana genome. Likewise, the putative function of $12-O-G l c-J A$ in storage and transport of jasmonates can be investigated (see 5.7). UGT76E1 and UGT76E2 showed a differential expression pattern in expression analyses (Supplemental figure 4). Therefore, localization studies might help to obtain hints to the relevance of the two enzymes in roots and shoots. This will also help to understand the role of 12-OH-JA and 12-O-Glc-JA in different plant tissues and may help to discover differential functions and regulations of the UGTs and the metabolic products.

In addition to jasmonates, oxylipins like 11-HHT, 9-HOT, and 13-HOT were glycosylated by all four candidate UGTs in this study. Oxylipins are involved in regulatory processes and stress responses in plants (see 1.1.1). Glycosylation of these FA-derived compounds might increase their solubility in the plant cell. As 11-HHT, 9-HOT, and 13-HOT are produced by the LOXpathway, an early interference with the pathway and its by-products is possible and may be part of the regulation of the oxylipin pool. However, oxylipin-glycosides have been found in L. paucicostata (Kai et al., 2010) but not in A. thaliana. In this respect, also the analytical methods have to be optimized to detect and identify glycosylated oxylipins in A. thaliana. With improved analytical methods, it may be possible to investigate the relevance of glycosylated oxylipins in the stress responses $e . g$. after wounding in A. thaliana. Such findings may enlarge the picture of the wound-response beside from jasmonates. Concerning jasmonates, this study has produced large amounts of $12-\mathrm{O}$-Glc-JA in the different activity assays. For $12-O$-Glc-JA, the authentic chemical standard is missing for quantitative and qualitative phytohormones measurements. 
Purifying 12-O-Glc-JA from the in vitro reactions of UGT76E1 and UGT76E2 will yield an authentic chemical standard for analytical methods in the future.

From the phylogenetic investigation, it was obvious that the group $\mathrm{H}$ of plant UGTs has not been investigated in detail and structural information are only available for UGT74F2. In contrast to UGT74F2, the enzymes in this work, UGT76E1, UGT76E2, UGT76E11, and UGT76E12, and UGT76B1 (von Saint Paul et al., 2011) seem to have substrate specificity towards hydrophobic or FA-derived substrates. Hence, crystal structures of candidate UGTs will bring detailed information about the reaction mechanism and the coordination of 12-OH-JA and oxylipins. A crystal structure would help to understand the function of the different functional groups that were identified as necessary for catalysis. Especially, UGT76E2 is a good candidate for this since it can be purified in high amounts from the expression cultures. 


\section{REFERENCES}

Albesa-Jové, D. and Guerin, M. E. (2016). The conformational plasticity of glycosyltransferases. Current Opinion in Structural Biology, 40, 23-32. doi: http://dx.doi.org/10.1016/j.sbi.2016.07.007.

Andreou, A., Brodhun, F. and Feussner, I. (2009). Biosynthesis of oxylipins in non-mammals. Progress in Lipid Research, 48, 148-170. doi: 10.1016/j.plipres.2009.02.002.

Arnold, M. D., Gruber, C., Floková, K., Miersch, O., Strnad, M., Novák, O., Wasternack, C. and Hause, B. (2016). The Recently Identified Isoleucine Conjugate of cis-12-OxoPhytodienoic Acid Is Partially Active in cis-12-Oxo-Phytodienoic Acid-Specific Gene Expression of Arabidopsis thaliana. PLoS ONE, 11, e0162829. doi: 10.1371/journal.pone.0162829.

Augustin, J. M., Drok, S., Shinoda, T., Sanmiya, K., Nielsen, J. K., Khakimov, B., Olsen, C. E., Hansen, E. H., Kuzina, V., Ekstrom, C. T., Hauser, T. and Bak, S. (2012). UDPglycosyltransferases from the UGT73C subfamily in Barbarea vulgaris catalyze sapogenin 3-O-glucosylation in saponin-mediated insect resistance. Plant Physiol, 160, 1881-1895. doi: 10.1104/pp.112.202747.

Ausubel, F. M., Brent, R. E., Kingston, D. D., Seidmann, J. R., Smith, J. A. and Struhl, K. (1993). Current Protocolls in Molecular Biology, Green Publishing Associates and John Wiley and Sons Inc., New York.

Bensinger, S. J. and Tontonoz, P. (2008). Integration of metabolism and inflammation by lipidactivated nuclear receptors. Nature, 454, 470-477. doi: 10.1038/nature07202.

Benveniste, I., Saito, T., Wang, Y., Kandel, S., Huang, H., Pinot, F., Kahn, R. A., Salaun, J.P. and Shimoji, M. (2006). Evolutionary relationship and substrate specificity of Arabidopsis thaliana fatty acid omega-hydroxylase. Plant Science, 170, 326-338.

Blée, E. (2002). Impact of phyto-oxylipins in plant defense. Trends in Plant Science, 7, 315-322.

Bligh, E. G. and Dyer, W. J. (1959). A rapid method of total lipid extraction and purification. Canadian journal of biochemistry and physiology, 37, 911-917.

Bowles, D., Lim, E.-K., Poppenberger, B. and Vaistij, F. n. E. (2006). GLYCOSYLTRANSFERASES OF LIPOPHILIC SMALL MOLECULES. Annual Review of Plant Biology, 57, 567-597. doi: doi:10.1146/annurev.arplant.57.032905.105429.

Britton, H. T. S. and Robinson, R. A. (1931). Universal buffer solutions and the dissociation constant of veronal. Journal of the Chemical Society (Resumed), 1456-1462.

Brown, C., Leijon, F. and Bulone, V. (2012). Radiometric and spectrophotometric in vitro assays of glycosyltransferases involved in plant cell wall carbohydrate biosynthesis. Nat Protoc, 7, 1634-1650. doi: 10.1038/nprot.2012.089.

Bruckhoff, V., Haroth, S., Feussner, K., König, S., Brodhun, F. and Feussner, I. (2016). Functional characterization of CYP94-genes and identification of a novel jasmonate catabolite in flowers. PLoS ONE, 11, e0159875. doi: 10.1371/journal.pone.0159875.

Caarls, L., Elberse, J., Awwanah, M., Ludwig, N. R., de Vries, M., Zeilmaker, T., Van Wees, S. C. M., Schuurink, R. C. and Van den Ackerveken, G. (2017). Arabidopsis JASMONATE-INDUCED OXYGENASES down-regulate plant immunity by hydroxylation and inactivation of the hormone jasmonic acid. Proceedings of the National Academy of Sciences, 114, 6388-6393. doi: 10.1073/pnas.1701101114.

Caldelari, D., Wang, G., Farmer, E. and Dong, X. (2011). Arabidopsis lox3 lox4 double mutants are male sterile and defective in global proliferative arrest. Plant Molecular Biology, 75, 25-33. doi: 10.1007/s11103-010-9701-9.

Campbell, J. A., Davies, G. J., Bulone, V. and Henrissat, B. (1997). A classification of nucleotide-diphospho-sugar glycosyltransferases based on amino acid sequence similarities. Biochemical Journal, 326, 929.

Chauvin, A., Caldelari, D., Wolfender, J.-L. and Farmer, E. E. (2012). Four 13-lipoxygenases contribute to rapid jasmonate synthesis in wounded Arabidopsis thaliana leaves: a role 
for lipoxygenase 6 in responses to long-distance wound signals. New Phytologist, 197, 566-575. doi: 10.1111/nph.12029.

Chehab, E. W., Kaspi, R., Savchenko, T., Rowe, H., Negre-Zakharov, F., Kliebenstein, D. and Dehesh, K. (2008). Distinct roles of jasmonates and aldehydes in plant-defense responses. PLoS ONE, 3, e1904.

Chini, A., Monte, I., Zamarreño, A. M., Hamberg, M., Lassueur, S., Reymond, P., Weiss, S., Stintzi, A., Schaller, A., Porzel, A., García-Mina, J. M. and Solano, R. (2018). An OPR3-independent pathway uses 4,5-didehydrojasmonate for jasmonate synthesis. Nature Chemical Biology, 14, 171-178. doi: 10.1038/nchembio.2540.

Clough, S. J. and Bent, A. F. (1998). Floral dip: a simplified method for Agrobacteriummediated transformation of Arabidopsis thaliana. Plant Journal, 16, 735-743.

Coleman, J., Mechteld, M. and Blake-Kalff, E. D. (1997). Detoxification of xenobiotics by plants: chemical modification and vacuolar compartmentation. Trends in plant science, 2, 141-151.

Davis, B. J. (1964). Disc electrophoresis-II method and application to human serum proteins. Annals of the New York Academy of Sciences, 121, 404-427.

Dean, J. V., Mohammed, L. A. and Fitzpatrick, T. (2005). The formation, vacuolar localization, and tonoplast transport of salicylic acid glucose conjugates in tobacco cell suspension cultures. Planta, 221, 287-296. doi: 10.1007/s00425-004-1430-3.

Dean, J. V., Shah, R. P. and Mohammed, L. A. (2003). Formation and vacuolar localization of salicylic acid glucose conjugates in soybean cell suspension cultures. Physiologia Plantarum, 118, 328-336.

Demole, E., Lederer, E. and Mercier, D. (1962). Isolement et détermination de la structure du jasmonate de méthyle, constituant odorant caractéristique de l'essence de jasmin. Helvetica Chimica Acta, 45, 675-685. doi: 10.1002/hlca.19620450233.

Dietz, K. J., Sauter, A., Wichert, K., Messdaghi, D. and Hartung, W. (2000). Extracellular $\beta$ glucosidase activity in barley involved in the hydrolysis of ABA glucose conjugate in leaves. Journal of Experimental Botany, 51, 937-944.

Dong, T., Xu, Z. Y., Park, Y., Kim, D. H., Lee, Y. and Hwang, I. (2014). Abscisic acid uridine diphosphate glucosyltransferases play a crucial role in abscisic acid homeostasis in Arabidopsis. Plant Physiol, 165, 277-289. doi: 10.1104/pp.114.239210.

Doyle, J. and Doyle, J. (1987). Genomic plant DNA preparation from fresh tissue-CTAB method. Phytochem Bull, 19, 11-15.

Duan, H., Huang, M.-Y., Palacio, K. and Schuler, M. A. (2005). Variations in CYP74B2 (hydroperoxide lyase) gene expression differentially affect hexenal signaling in the Columbia and Landsberg erecta ecotypes of Arabidopsis. Plant Physiology, 139, 15291544.

Farmer, E. E. (2007). Plant biology: Jasmonate perception machines. Nature, 448, 659-660.

Feussner, I. and Wasternack, C. (2002). The lipoxygenase pathway. Annual Review of Plant Biology, 53, 275-297. doi: DOI: 10.1146/annurev.arplant.53.100301.135248.

Ford, C. M., Boss, P. K. and Høj, P. B. (1998). Cloning and characterization of Vitis vinifera UDP-Glucose: flavonoid 3-O-glucosyltransferase, a homologue of the enzyme encoded by the maize bronze-1 Locus that may primarily serve to glucosylate anthocyanidins in vivo. Journal of Biological Chemistry, 273, 9224-9233.

Gasperini, D., Chetelat, A., Acosta, I. F., Goossens, J., Pauwels, L., Goossens, A., Dreos, R., Alfonso, E. and Farmer, E. E. (2015). Multilayered Organization of Jasmonate Signalling in the Regulation of Root Growth. PLoS Genet, 11, e1005300. doi: 10.1371/journal.pgen.1005300.

George Thompson, A. M., Iancu, C. V., Neet, K. E., Dean, J. V. and Choe, J. Y. (2017). Differences in salicylic acid glucose conjugations by UGT74F1 and UGT74F2 from Arabidopsis thaliana. Sci Rep, 7, 46629. doi: 10.1038/srep46629.

Ghareeb, H., Becker, A., Iven, T., Feussner, I. and Schirawski, J. (2011). Sporisorium reilianum Infection Changes Inflorescence and Branching Architectures of Maize. Plant Physiology, 156, 2037-2052. doi: 10.1104/pp.111.179499. 
Gidda, S. K., Miersch, O., Levitin, A., Schmidt, J., Wasternack, C. and Varin, L. (2003). Biochemical and molecular characterization of a hydroxyjasmonate sulfotransferase from Arabidopsis thaliana. J. Biol. Chem., 278, 17895-17900.

Glauser, G., Dubugnon, L., Mousavi, S. A. R., Rudaz, S., Wolfender, J.-L. and Farmer, E. E. (2009). Velocity estimates for signal propagation leading to systemic jasmonic acid accumulation in wounded Arabidopsis. Journal of Biological Chemistry, 284, 3450634513. doi: 10.1074/jbc.M109.061432.

Guo, Q., Major, I. T. and Howe, G. A. (2018). Resolution of growth-defense conflict: mechanistic insights from jasmonate signaling. Current Opinion in Plant Biology, 44, 7281. doi: https://doi.org/10.1016/j.pbi.2018.02.009.

Haroth, S. (2014). Catabolism of jasmonates. Vol. Master Göttingen.

Heitz, T., Widemann, E., Lugan, R., Miesch, L., Ullmann, P., Desaubry, L., Holder, E., Grausem, B., Kandel, S., Miesch, M., Werck-Reichhart, D. and Pinot, F. (2012). Cytochromes P450 CYP94C1 and CYP94B3 catalyze two successive oxidation steps of plant hormone jasmonoyl-isoleucine for catabolic turnover. Journal of Biological Chemistry, 287, 6296-6306. doi: 10.1074/jbc.M111.316364.

Hofer, R., Boachon, B., Renault, H., Gavira, C., Miesch, L., Iglesias, J., Ginglinger, J. F., Allouche, L., Miesch, M., Grec, S., Larbat, R. and Werck-Reichhart, D. (2014). Dual function of the cytochrome P450 CYP76 family from Arabidopsis thaliana in the metabolism of monoterpenols and phenylurea herbicides. Plant Physiol, 166, 1149-1161. doi: 10.1104/pp.114.244814.

Hou, B., Lim, E. K., Higgins, G. S. and Bowles, D. J. (2004). $N$-glucosylation of cytokinins by glycosyltransferases of Arabidopsis thaliana. J Biol Chem, 279, 47822-47832. doi: 10.1074/jbc.M409569200.

Howe, G. A. and Jander, G. (2008). Plant immunity to insect herbivores. Annual Review of Plant Biology, 59, 41-66. doi: 10.1146/annurev.arplant.59.032607.092825.

Huang, F. C., Hinkelmann, J. and Schwab, W. (2015). Glucosylation of aroma chemicals and hydroxy fatty acids. J Biotechnol, 216, 100-109. doi: 10.1016/j.jbiotec.2015.10.011.

Huang, X., Zhu, G.-q., Liu, Q., Chen, L., Li, Y.-J. and Hou, B.-K. (2018). Modulation of plant salicylic acid-associated immune responses via glycosylation of dihydroxybenzoic acids. Plant Physiology. doi: 10.1104/pp.17.01530.

Ishimaru, Y., Hayashi, K.-i., Suzuki, T., Fukaki, H., Prusinska, J., Meesters, C., Quareshy, M., Egoshi, Y., Matsuura, H., Takahashi, K., Kato, N., Kombrink, E., Napier, R. M., Hayashi, K. and Ueda, M. (2018). Jasmonic acid inhibits auxin-induced lateral rooting independently of the CORONATINE INSENSITIVE 1 receptor. Plant Physiology, 177, 704-1716. doi: 10.1104/pp.18.00357.

Iven, T., Feussner, K., Herrfurth, C. and Herbig, E. (2014). Signalstoffe der pflanzlichen Abwehr. LABO Life sciences, 12, 43-47.

Jackson, R. G., Lim, E. K., Li, Y., Kowalczyk, M., Sandberg, G., Hoggett, J., Ashford, D. A. and Bowles, D. J. (2001). Identification and biochemical characterization of an Arabidopsis indole-3-acetic acid glucosyltransferase. J Biol Chem, 276, 4350-4356. doi: 10.1074/jbc.M006185200.

Jin, S. H., Ma, X. M., Han, P., Wang, B., Sun, Y. G., Zhang, G. Z., Li, Y. J. and Hou, B. K. (2013). UGT74D1 is a novel auxin glycosyltransferase from Arabidopsis thaliana. PLoS One, 8, e61705. doi: 10.1371/journal.pone.0061705.

Kaever, A. (2014). Development of a statistical framework for mass spectrometry data analysis in untargeted Metabolomics studies. Vol. Dissertation Göttingen.

Kaever, A., Landesfeind, M., Feussner, K., Morgenstern, B., Feussner, I. and Meinicke, P. (2014). Meta-analysis of pathway enrichment: combining independent and dependent omics data sets. PLoS ONE, 9, e89297. doi: 10.1371/journal.pone.0089297.

Kai, K., Akaike, R., Iida, K., Yokoyama, M. and Watanabe, N. (2010). C14-Oxylipin glucosides isolated from Lemna paucicostata. Phytochemistry, In Press, Corrected Proof.

Kellenberger, E. (1968). Studies on the morphopoiesis of the head of phage T-even: V. The components of the T4 capsid and of other, capsid-related structures. Virology, 34, 549561. 
Kessler, A. and Baldwin, I. T. (2001). Defensive Function of Herbivore-Induced Plant Volatile Emissions in Nature. Science, 291, 2141-2144. doi: 10.1126/science.291.5511.2141.

Khan, B. R., Wherritt, D. J., Huhman, D., Sumner, L. W., Chapman, K. D. and Blancaflor, E. B. (2016). Malonylation of glucosylated $N$-lauroylethanolamine: a new pathway that determines $N$-acylethanolamine metabolic fate in plants. J Biol Chem, 291, 27112-27121. doi: 10.1074/jbc.M116.751065.

Kilian, J., Whitehead, D., Horak, J., Wanke, D., Weinl, S., Batistic, O., D'Angelo, C., Bornberg-Bauer, E., Kudla, J. and Harter, K. (2007). The AtGenExpress global stress expression data set: protocols, evaluation and model data analysis of UV-B light, drought and cold stress responses. Plant J, 50, 347-363. doi: 10.1111/j.1365-313X.2007.03052.x.

Kim, J. H., Kim, B. G., Park, Y., Ko, J. H., Lim, C. E., Lim, J., Lim, Y. and Ahn, J. H. (2006). Characterization of flavonoid 7-O-glucosyltransferase from Arabidopsis thaliana. Biosci Biotechnol Biochem, 70, 1471-1477. doi: 10.1271/bbb.60006.

Kitaoka, N., Kawaide, H., Amano, N., Matsubara, T., Nabeta, K., Takahashi, K. and Matsuura, H. (2014). CYP94B3 activity against jasmonic acid amino acid conjugates and the elucidation of 12-O- $\beta$-glucopyranosyl-jasmonoyl- $L$-isoleucine as an additional metabolite. Phytochemistry, 99, 6-13. 9oi: http://dx.doi.org/10.1016/j.phytochem.2013.12.019.

Kitaoka, N., Matsubara, T., Sato, M., Takahashi, K., Wakuta, S., Kawaide, H., Matsui, H., Nabeta, K. and Matsuura, H. (2011). Arabidopsis CYP94B3 encodes jasmonyl-Lisoleucine 12-hydroxylase, a key enzyme in the oxidative catabolism of jasmonate. Plant and Cell Physiology, 52, 1757-1765. doi: 10.1093/pcp/pcr110.

Koo, A. J. (2018). Metabolism of the plant hormone jasmonate: a sentinel for tissue damage and master regulator of stress response. Phytochemistry Reviews, 17, 51-80. doi: 10.1007/s11101-017-9510-8.

Koo, A. J., Thireault, C., Zemelis, S., Poudel, A. N., Zhang, T., Kitaoka, N., Brandizzi, F., Matsuura, H. and Howe, G. A. (2014). Endoplasmic reticulum-associated inactivation of the hormone jasmonoyl-L-isoleucine by multiple members of the cytochrome P450 94 family in Arabidopsis. Journal of Biological Chemistry, 289, 29728-29738,. doi: 10.1074/jbc.M114.603084.

Koo, A. J. K., Chung, H. S., Kobayashi, Y. and Howe, G. A. (2006). Identification of a peroxisomal acyl-activating enzyme involved in the biosynthesis of jasmonic acid in Arabidopsis. J. Biol. Chem., 281, 33511-33520.

Koo, A. J. K., Cooke, T. F. and Howe, G. A. (2011). Cytochrome P450 CYP94B3 mediates catabolism and inactivation of the plant hormone jasmonoyl-L-isoleucine. Proceedings of the National Academy of Sciences, 108, 9298-9303. doi: 10.1073/pnas.1103542108.

Koo, A. J. K. and Howe, G. A. (2009). The wound hormone jasmonate. Phytochemistry, 70, $1571-1580$

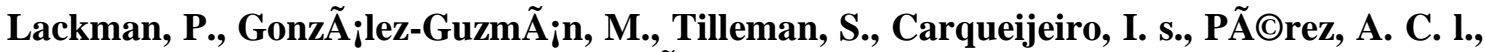
Moses, T., Seo, M., Kanno, Y., HÃ âkkinen, S. T., Van Montagu, M. C. E., Thevelein, J. M., Maaheimo, H., Oksman-Caldentey, K.-M., Rodriguez, P. L., Rischer, H. and Goossens, A. (2011). Jasmonate signaling involves the abscisic acid receptor PYL4 to regulate metabolic reprogramming in Arabidopsis and tobacco. Proceedings of the National Academy of Sciences, 108, 5891-5896. doi: 10.1073/pnas.1103010108.

Laemmli, U. K. (1970). Cleavage of Structural Proteins during the Assembly of the Head of Bacteriophage T4. Nature, 227, 680. doi: 10.1038/227680a0.

Lairson, L. L., Henrissat, B., Davies, G. J. and Withers, S. G. (2008). Glycosyltransferases: structures, functions, and mechanisms. Annu Rev Biochem, 77, 521-555. doi: 10.1146/annurev.biochem.76.061005.092322.

Lee, S., Yang, D. S., Uppalapati, S. R., Sumner, L. and Mysore, K. (2013). Suppression of plant defense responses by extracellular metabolites from Pseudomonas syringae pv. tabaci in Nicotiana benthamiana. BMC Plant Biology, 13, 65.

Lesk, A. M. (1995). Systematic representation of protein folding patterns. Journal of molecular graphics, 13, 159-164. 
Li, Q., Yu, H.-M., Meng, X.-F., Lin, J.-S., Li, Y.-J. and Hou, B.-K. (2018). Ectopic expression of glycosyltransferase UGT76E11 increases flavonoid accumulation and enhances abiotic stress tolerance in Arabidopsis. Plant Biology, 20, 10-19. doi: 10.1111/plb.12627.

Li, Q., Zheng, J., Li, S., Huang, G., Skilling, S. J., Wang, L., Li, L., Li, M., Yuan, L. and Liu, P. (2017). Transporter-Mediated Nuclear Entry of Jasmonoyl-Isoleucine Is Essential for Jasmonate Signaling. Molecular Plant. doi: http://dx.doi.org/10.1016/j.molp.2017.01.010.

Lim, E. K., Ashford, D. A., Hou, B., Jackson, R. G. and Bowles, D. J. (2004). Arabidopsis glycosyltransferases as biocatalysts in fermentation for regioselective synthesis of diverse quercetin glucosides. Biotechnol Bioeng, 87, 623-631. doi: 10.1002/bit.20154.

Lim, E. K., Doucet, C. J., Li, Y., Elias, L., Worrall, D., Spencer, S. P., Ross, J. and Bowles, D. J. (2002). The activity of Arabidopsis glycosyltransferases toward salicylic acid, 4hydroxybenzoic acid, and other benzoates. J Biol Chem, 277, 586-592. doi: 10.1074/jbc.M109287200.

Lim, E. K., Li, Y., Parr, A., Jackson, R., Ashford, D. A. and Bowles, D. J. (2001). Identification of glucosyltransferase genes involved in sinapate metabolism and lignin synthesis in Arabidopsis. J Biol Chem, 276, 4344-4349. doi: 10.1074/jbc.M007263200.

Louis, J., Lorenc-Kukula, K., Singh, V., Reese, J., Jander, G. and Shah, J. (2010). Antibiosis against the green peach aphid requires the Arabidopsis thaliana MYZUS PERSICAEINDUCED LIPASE1 gene. Plant J, 64, 800-811. doi: 10.1111/j.1365313X.2010.04378.X.

Mackenzie, P. I., Owens, I. S., Burchell, B., Bock, K. W., Bairoch, A., Belanger, A., FournelGigleux, S., Green, M., Hum, D. W. and Iyanagi, T. (1997). The UDP glycosyltransferase gene superfamily: recommended nomenclature update based on evolutionary divergence. Pharmacogenetics, 7, 255-269.

Matsui, K. (2006). Green leaf volatiles: hydroperoxide lyase pathway of oxylipin metabolism. Current Opinion in Plant Biology, 9, 274-280.

Matyash, V., Liebisch, G., Kurzchalia, T. V., Shevchenko, A. and Schwudke, D. (2008). Lipid extraction by methyl-tert-butyl ether for high-throughput lipidomics. Journal of Lipid Research, 49, 1137-1146. doi: 10.1194/jlr.D700041-JLR200.

Mene-Saffrane, L., Esquerre-Tugaye, M. T. and Fournier, J. (2003). Constitutive expression of an inducible lipoxygenase in transgenic tobacco decreases susceptibillity to Phytophthora parasitica var. nicotianae. Molecular Breeding, 12, 271-282.

Meng, M., Geisler, M., Johansson, H., Harholt, J., Scheller, H. V., Mellerowicz, E. J. and Kleczkowski, L. A. (2009). UDP-glucose pyrophosphorylase is not rate limiting, but is essential in Arabidopsis. Plant Cell Physiol, 50, 998-1011. doi: 10.1093/pcp/pcp052.

Miersch, O., Neumerkel, J., Dippe, M., Stenzel, I. and Wasternack, C. (2008). Hydroxylated jasmonates are commonly occurring metabolites of jasmonic acid and contribute to a partial switch-off in jasmonate signaling. New Phytologist, 177, 114-127.

Miersch, O., Preiss, A., Sembdner, G. and Schreiber, K. (1987). (+)-7-iso-jasmonic acid and related compounds from Botryodiplodia theobromae. Phytochemistry, 26, 1037-1039. doi: 10.1016/S0031-9422(00)82345-6.

Mizutani, M. and Sato, F. (2010). Unusual P450 reactions in plant secondary metabolism. Archives of Biochemistry and Biophysics, In Press, Accepted Manuscript.

Monte, I., Ishida, S., Zamarreño, A. M., Hamberg, M., Franco-Zorrilla, J. M., GarcíaCasado, G., Gouhier-Darimont, C., Reymond, P., Takahashi, K., García-Mina, J. M., Nishihama, R., Kohchi, T. and Solano, R. (2018). Ligand-receptor co-evolution shaped the jasmonate pathway in land plants. Nature Chemical Biology. doi: 10.1038/s41589-018-0033-4.

Mosblech, A. (2010). Requirement of phosphoinositol-derived signals in the wounding response of Arabidopsis thaliana. Vol. Dissertation Göttingen.

Mugford, S. G., Yoshimoto, N., Reichelt, M., Wirtz, M., Hill, L., Mugford, S. T., Nakazato, Y., Noji, M., Takahashi, H., Kramell, R., Gigolashvili, T., Flugge, U. I., Wasternack, C., Gershenzon, J., Hell, R., Saito, K. and Kopriva, S. (2009). Disruption of adenosine5'-phosphosulfate kinase in Arabidopsis reduces levels of sulfated secondary metabolites. Plant Cell, 21, 910-927. doi: 10.1105/tpc.109.065581. 
Nahlik, K., Dumkow, M., Bayram, Ö., Helmstaedt, K., Busch, S., Valerius, O., Gerke, J., Hoppert, M., Schwier, E., Opitz, L., Westermann, M., Grond, S., Feussner, K., Göbel, C., Kaever, A., Meinicke, P., Feussner, I. and Braus, G. H. (2010). The COP9 signalosome mediates transcriptional and metabolic response to hormones, oxidative stress protection and cell wall rearrangement during fungal development. Molecular Microbiology, 78, 964-979. doi: 10.1111/j.1365-2958.2010.07384.x.

Nakamura, Y., Mithofer, A., Kombrink, E., Boland, W., Hamamoto, S., Uozumi, N., Tohma, K. and Ueda, M. (2011). 12-Hydroxyjasmonic Acid Glucoside Is a COI1-JAZIndependent Activator of Leaf-Closing Movement in Samanea saman. Plant Physiology, 155, 1226-1236. doi: 10.1104/pp.110.168617.

Nguyen, C. T., Martinoia, E. and Farmer, E. E. (2017). Emerging Jasmonate Transporters. Molecular Plant. doi: http://dx.doi.org/10.1016/j.molp.2017.03.007.

Offen, W., Martinez-Fleites, C., Yang, M., Kiat-Lim, E., Davis, B. G., Tarling, C. A., Ford, C. M., Bowles, D. J. and Davies, G. J. (2006). Structure of a flavonoid glucosyltransferase reveals the basis for plant natural product modification. The EMBO journal, 25, 1396-1405.

Onate-Sanchez, L. and Vicente-Carbajosa, J. (2008). DNA-free RNA isolation protocols for Arabidopsis thaliana, including seeds and siliques. BMC Res Notes, 1, 93. doi: 10.1186/1756-0500-1-93.

Ornstein, L. (1964). Disc electrophoresis-i background and theory. Annals of the New York Academy of Sciences, 121, 321-349.

Ovádi, J. and Saks, V. (2004). On the origin of intracellular compartmentation and organized metabolic systems. Molecular and cellular biochemistry, 256, 5-12.

Park, J.-H., Halitschke, R., Kim, H. B., Baldwin, I. T., Feldmann, K. A. and Feyereisen, R. (2002). A knock-out mutation in allene oxide synthase results in male sterility and defective wound signal transduction in Arabidopsis due to a block in jasmonic acid biosynthesis. The Plant Journal, 31, 1-12.

Pauwels, L., Barbero, G. F., Geerinck, J., Tilleman, S., Grunewald, W., Perez, A. C., Chico, J. M., Bossche, R. V., Sewell, J., Gil, E., Garcia-Casado, G., Witters, E., Inze, D., Long, J. A., De Jaeger, G., Solano, R. and Goossens, A. (2010). NINJA connects the co-repressor TOPLESS to jasmonate signalling. Nature, 464, 788-791.

Pieterse, C. M. J., Leon-Reyes, A., Van der Ent, S. and Van Wees, S. C. M. (2009). Networking by small-molecule hormones in plant immunity. Nature Chemical Biology, 5, 308-316. doi: 10.1038/nchembio. 164.

Pieterse, C. M. J., Poelman, E., Van Wees, S. C. M. and Dicke, M. (2013). Induced plant responses to microbes and insects. Frontiers in Plant Science, $\mathbf{4}$. doi: 10.3389/fpls.2013.00475.

Rehman, H. M., Nawaz, M. A., Shah, Z. H., Ludwig-Muller, J., Chung, G., Ahmad, M. Q., Yang, S. H. and Lee, S. I. (2018). Comparative genomic and transcriptomic analyses of Family-1 UDP glycosyltransferase in three Brassica species and Arabidopsis indicates stress-responsive regulation. Sci Rep, 8, 1875. doi: 10.1038/s41598-018-19535-3.

Ross, J., Li, Y., Lim, E.-K. and Bowles, D. J. (2001). Higher plant glycosyltransferases. Genome Biology, 2, reviews3004.3001. doi: 10.1186/gb-2001-2-2-reviews3004.

Saito, H., Oikawa, T., Hamamoto, S., Ishimaru, Y., Kanamori-Sato, M., Sasaki-Sekimoto, Y., Utsumi, T., Chen, J., Kanno, Y., Masuda, S., Kamiya, Y., Seo, M., Uozumi, N., Ueda, M. and Ohta, H. (2015). The jasmonate-responsive GTR1 transporter is required for gibberellin-mediated stamen development in Arabidopsis. Nature Communications, 6, 6095. doi: 10.1038/ncomms7095.

Sambrook, J., Fritsch, E. F. and Maniatis, T. (1989). Molecular cloning: a laboratory manual, 2nd edn. edn. Cold Spring Harbor Laboratory, Cold Spring Harbor NY.

Seto, Y., Hamada, S., Ito, H., Masuta, C., Matsui, H., Nabeta, K. and Matsuura, H. (2011). Tobacco salicylic acid glucosyltransferase is active toward tuberonic acid (12hydroxyjasmonic acid) and is induced by mechanical wounding stress. Biosci Biotechnol Biochem, 75, 2316-2320. doi: 10.1271/bbb.110454.

Seto, Y., Hamada, S., Matsuura, H., Matsushige, M., Satou, C., Takahashi, K., Masuta, C., Ito, H., Matsui, H. and Nabeta, K. (2009). Purification and cDNA cloning of a wound 
inducible glucosyltransferase active toward 12-hydroxy jasmonic acid. Phytochemistry, 70, 370-379. doi: http://dx.doi.org/10.1016/j.phytochem.2009.01.004.

Smirnova, E., Marquis, V., Poirier, L., Aubert, Y., Zumsteg, J., Ménard, R., Miesch, L. and Heitz, T. (2017). Jasmonic Acid Oxidase 2 (JAO2) hydroxylates jasmonic acid and represses basal defense and resistance responses against Botrytis cinerea infection. Molecular Plant, 10, 1159-1173. doi: https://doi.org/10.1016/j.molp.2017.07.010.

Somssich, I. E., Wernert, P., Kiedrowski, S. and Hahlbrock, K. (1996). Arabidopsis thaliana defense-related protein ELI3 is an aromatic alcohol: NADP+ oxidoreductase. Proceedings of the National Academy of Sciences, 93, 14199-14203.

Song, J. T. (2005). Biochemical characterization of an Arabidopsis glucosyltransferase with high activity toward jasmonic acid. Journal of Plant Biology, 48, 422-428.

Staswick, P. E. and Tiryaki, I. (2004). The oxylipin signal jasmonic acid is activated by an enzyme that conjugates it to isoleucine in Arabidopsis. The Plant Cell, 16, 2117-2127. doi: DOI 10.1105/tpc.104.023549.

Stintzi, A. and Browse, J. (2000). The Arabidopsis male-sterile mutant, opr3, lacks the 12oxophytodienoic acid reductase required for jasmonate synthesis. Proceedings of the National Academy of Sciences of the United States of America, 97, 10625-10630.

Studier, F. W. (2005). Protein production by auto-induction in high-density shaking cultures. Protein Expression and Purification, 41, 207-234. doi: http://dx.doi.org/10.1016/j.pep.2005.01.016.

Suzuki, H., Hayase, H., Nakayama, A., Yamaguchi, I., Asami, T. and Nakajima, M. (2007). Identification and characterization of an Ipomoea nil glucosyltransferase which metabolizes some phytohormones. Biochemical and Biophysical Research Communications, 361, 980-986. doi: http://dx.doi.org/10.1016/j.bbrc.2007.07.147.

Swiatek, A., Dongen, W. V., Esmans, E. L. and Onckelen, H. V. (2004). Metabolic fate of jasmonates in Tobacco bright yellow-2 cells. Plant Physiology, 135, 161-172.

Tanaka, K., Hayashi, K., Natsume, M., Kamiya, Y., Sakakibara, H., Kawaide, H. and Kasahara, H. (2014). UGT74D1 catalyzes the glucosylation of 2-oxindole-3-acetic acid in the auxin metabolic pathway in Arabidopsis. Plant Cell Physiol, 55, 218-228. doi: 10.1093/pcp/pct173.

Theodoulou, F. L., Job, K., Slocombe, S. P., Footitt, S., Holdsworth, M., Baker, A., Larson, T. R. and Graham, I. A. (2005). Jasmonic acid levels are reduced in COMATOSE ATPbinding cassette transporter mutants. Implications for transport of jasmonate precursors into peroxisomes. Plant Physiology, 137, 835-840.

Ueda, J. and Kato, J. (1980). Isolation and Identification of a Senescence-promoting Substance from Wormwood (Artemisia absinthium L.). Plant Physiology, 66, 246-249. doi: 10.1104/pp.66.2.246.

Vogt, T. and Jones, P. (2000). Glycosyltransferases in plant natural product synthesis: characterization of a supergene family. Trends in Plant Science, 5, 380-386. doi: http://dx.doi.org/10.1016/S1360-1385(00)01720-9.

von Malek, B., van der Graaff, E., Schneitz, K. and Keller, B. (2002). The Arabidopsis malesterile mutant $d d e 2-2$ is defective in the ALLENE OXIDE SYNTHASE gene encoding one of the key enzymes of the jasmonic acid biosynthesis pathway. Planta, 216, 187-192.

von Saint Paul, V., Zhang, W., Kanawati, B., Geist, B., Faus-Kessler, T., Schmitt-Kopplin, P. and Schäffner, A. R. (2011). The Arabidopsis Glucosyltransferase UGT76B1 Conjugates Isoleucic Acid and Modulates Plant Defense and Senescence. The Plant Cell, 23, 4124-4145. doi: tpc.111.088443 [pii]

10.1105/tpc.111.088443.

Wakuta, S., Hamada, S., Ito, H., Matsuura, H., Nabeta, K. and Matsui, H. (2010). Identification of a beta-glucosidase hydrolyzing tuberonic acid glucoside in rice (Oryza sativa L.). Phytochemistry, 71, 1280-1288. doi: 10.1016/j.phytochem.2010.04.025.

Wang, X. (2009). Structure, mechanism and engineering of plant natural product glycosyltransferases. FEBS Lett, 583, 3303-3309. doi: 10.1016/j.febslet.2009.09.042.

Wang, Z.-P., Xing, H.-L., Dong, L., Zhang, H.-Y., Han, C.-Y., Wang, X.-C. and Chen, Q.-J. (2015). Egg cell-specific promoter-controlled CRISPR/Cas9 efficiently generates 
homozygous mutants for multiple target genes in Arabidopsis in a single generation. Genome Biology, 16. doi: 10.1186/s13059-015-0715-0.

Wasternack, C. (2015). How jasmonates earned their laurels: Past and present. Journal of Plant Growth Regulation, 1-34. doi: 10.1007/s00344-015-9526-5.

Wasternack, C. and Feussner, I. (2018). The oxylipin pathways: Biochemistry and function. Annual Review of Plant Biology, 69, 363-386. doi: 10.1146/annurev-arplant-042817040440.

Wasternack, C. and Hause, B. (2013). Jasmonates: biosynthesis, perception, signal transduction and action in plant stress response, growth and development. An update to the 2007 review in Annals of Botany. Annals of Botany, 111, 1021-1058. doi: 10.1093/aob/mct067.

Wasternack, C., Miersch, O., Kramell, R., Hause, B., Ward, J., Beale, M., Boland, W., Parthier, B. and Feussner, I. (1998). Jasmonic acid: biosynthesis, signal transduction, gene expression. Fett/Lipid, 100, 139-146.

Wasternack, C. and Song, S. (2017). Jasmonates: biosynthesis, metabolism, and signaling by proteins activating and repressing transcription. Journal of Experimental Botany, 68, 1303-1321. doi: 10.1093/jxb/erw443.

Weber, H., Vick, B. A. and Farmer, E. E. (1997). Dinor-oxo-phytodienoic acid: A new hexadecanoid signal in the jasmonate family. Proceedings of the National Academy of Sciences of the United States of America, 94, 10473-10478.

Weichert, H., Stenzel, I., Berndt, E., Wasternack, C. and Feussner, I. (1999). Metabolic profiling of oxylipins upon salicylate treatment in barley leaves - preferential induction of the reductase pathway by salicylate. FEBS Letters, 464, 133-137.

Weigel, D. and Glazebrook, J. (2002). Arabidopsis: a laboratory manual, CSHL Press.

Westfall, C. S., Sherp, A. M., Zubieta, C., Alvarez, S., Schraft, E., Marcellin, R., Ramirez, L. and Jez, J. M. (2016). Arabidopsis thaliana GH3.5 acyl acid amido synthetase mediates metabolic crosstalk in auxin and salicylic acid homeostasis. Proceedings of the National Academy of Sciences, 113, 13917-13922. doi: 10.1073/pnas.1612635113.

Westfall, C. S., Zubieta, C., Herrmann, J., Kapp, U., Nanao, M. H. and Jez, J. M. (2012). Structural Basis for Prereceptor Modulation of Plant Hormones by GH3 Proteins. Science, 336, 1708-1711. doi: 10.1126/science.1221863.

Widemann, E., Grausem, B., Renault, H., Pineau, E., Heinrich, C., Lugan, R., Ullmann, P., Miesch, L., Aubert, Y., Miesch, M., Heitz, T. and Pinot, F. (2015). Sequential oxidation of jasmonoyl-phenylalanine and jasmonoyl-isoleucine by multiple cytochrome P450 of the CYP94 family through newly identified aldehyde intermediates. Phytochemistry, 117, 388-399. doi: http://dx.doi.org/10.1016/j.phytochem.2015.06.027.

Widemann, E., Miesch, L., Lugan, R., Holder, E., Heinrich, C., Aubert, Y., Miesch, M., Pinot, F. and Heitz, T. (2013). The Amidohydrolases IAR3 and ILL6 contribute to jasmonoyl-isoleucine hormone turnover and generate 12-hydroxyjasmonic acid upon wounding in Arabidopsis leaves. Journal of Biological Chemistry, 288, 31701-31714. doi: 10.1074/jbc.M113.499228.

Widemann, E., Smirnova, E., Aubert, Y., Miesch, L. and Heitz, T. (2016). Dynamics of jasmonate metabolism upon flowering and across leaf stress responses in Arabidopsis thaliana. Plants, $\mathbf{5}, 4$.

Winter, D., Vinegar, B., Nahal, H., Ammar, R., Wilson, G. V. and Provart, N. J. (2007). An "Electronic Fluorescent Pictograph" browser for exploring and analyzing large-scale biological data sets. PLOS ONE, 2, e718. doi: 10.1371/journal.pone.0000718.

Woodward, A. W. and Bartel, B. (2005). Auxin: regulation, action, and interaction. Ann Bot, 95, 707-735. doi: 10.1093/aob/mci083.

Wu, J. and Baldwin, I. T. (2010). New Insights into Plant Responses to the Attack from Insect Herbivores. Annual Review of Genetics, 44, 1-24. doi: doi:10.1146/annurev-genet102209-163500.

Xing, H.-L., Dong, L., Wang, Z.-P., Zhang, H.-Y., Han, C.-Y., Liu, B., Wang, X. and Chen, Q.-J. (2014). A CRISPR/Cas9 toolkit for multiplex genome editing in plants. BMC Plant Biology, 14, 327. 
Xu, Y., Chang, P. F. L., Liu, D., Narasimhan, M. L., Raghothama, K. G., Hasegawa, P. M. and Bressan, R. A. (1994). Plant defense genes are synergistically induced by ethylene and methyl jasmonate. The Plant Cell, 6, 1077-1085.

Yoshida, Y., Sano, R., Wada, T., Takabayashi, J. and Okada, K. (2009). Jasmonic acid control of GLABRA3 links inducible defense and trichome patterning in Arabidopsis. Development, 136, 1039-1048. doi: 10.1242/dev.030585.

Yoshihara, T., Omir, E.-S. A., Koshino, H., Sakamura, S., Kkuta, Y. and Koda, Y. (2014). Structure of a Tuber-inducing Stimulus from Potato Leaves (Solanum tuberosum L.). Agricultural and Biological Chemistry, 53, 2835-2837. doi: 10.1080/00021369.1989.10869712.

Zhang, T., Poudel, A. N., Jewell, J. B., Kitaoka, N., Staswick, P., Matsuura, H. and Koo, A. J. (2016). Hormone crosstalk in wound stress response: wound-inducible amidohydrolases can simultaneously regulate jasmonate and auxin homeostasis in Arabidopsis thaliana. Journal of Experimental Botany, 67, 2107-2120. doi: 10.1093/jxb/erv521.

Zhu, Z., An, F., Feng, Y., Li, P., Xue, L., A, M., Jiang, Z., Kim, J. M., To, T. K., Li, W., Zhang, X., Yu, Q., Dong, Z., Chen, W. Q., Seki, M., Zhou, J. M. and Guo, H. (2011). Derepression of ethylene-stabilized transcription factors (EIN3/EIL1) mediates jasmonate and ethylene signaling synergy in Arabidopsis. Proc Natl Acad Sci U S A, 108, 12539-12544. doi: 10.1073/pnas.1103959108. 


\section{SUPPLEMENTAL DATA}

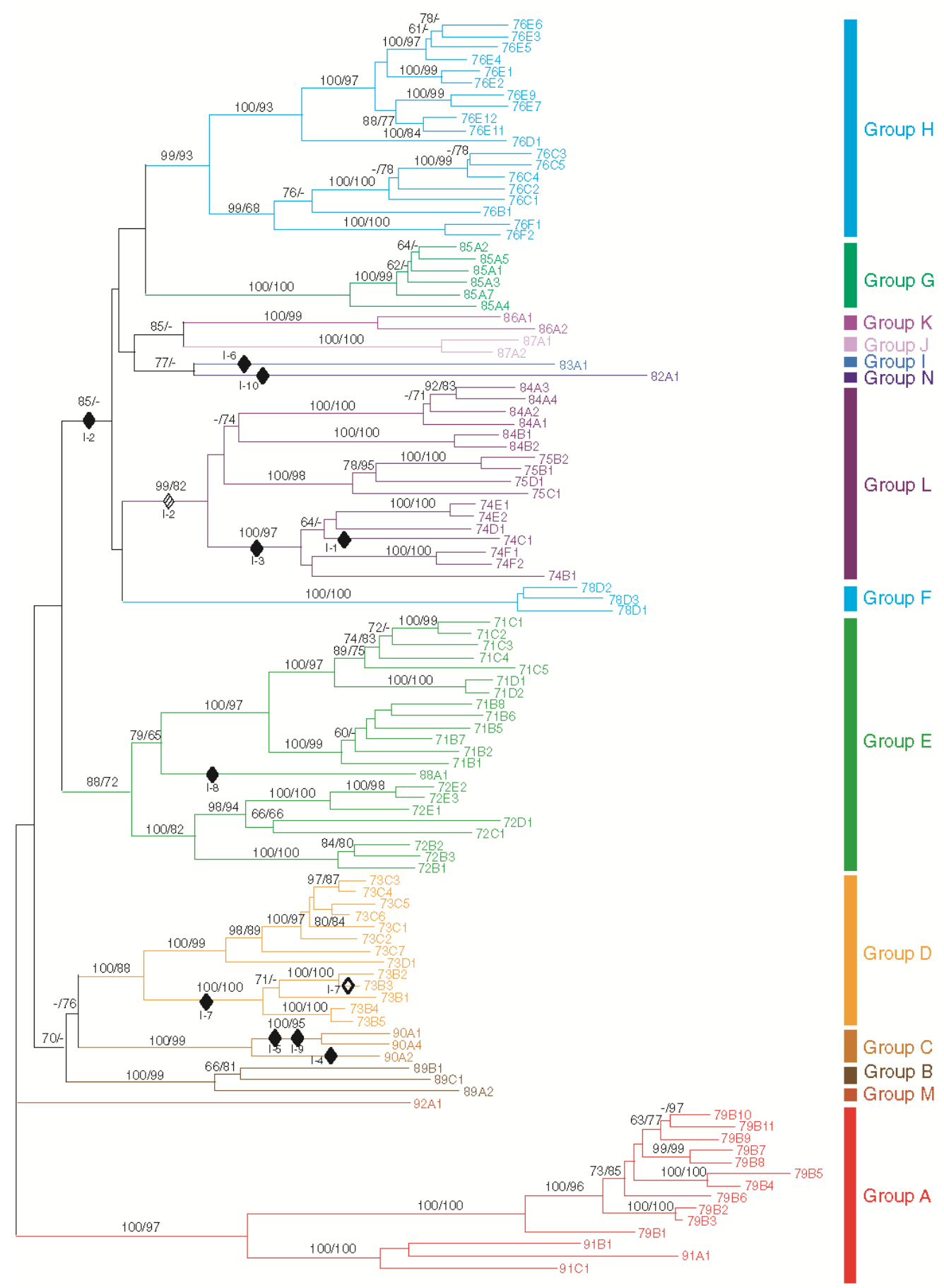

\section{Supplemental figure 1: Phylogenetic tree of glycosyltransferases of $A$. thaliana}

Phylogenetic analysis of 107 UGTs in A. thaliana. "Neighbor-joining and parsimony-based analysis of nine conserved amino acid sequences. Bootstrap values over $60 \%$ are indicated above the nodes, with the number on the left indicating neighbor-joining and that on the right indicating parsimony. Dashes indicate bootstrap values under 60\%. Hypothetical intron gains and losses are indicated by diamonds with the intron number (I). Postulated intron gains are indicated by filled diamonds, intron losses by unfilled diamonds and the questionable intron loss by a striped diamond." Colors indicate phylogenetic groups (AN) with a bootstrap support greater than $90 \%$ in the distance analysis. Figure and text taken from Ross $e t$ al., 2001. 


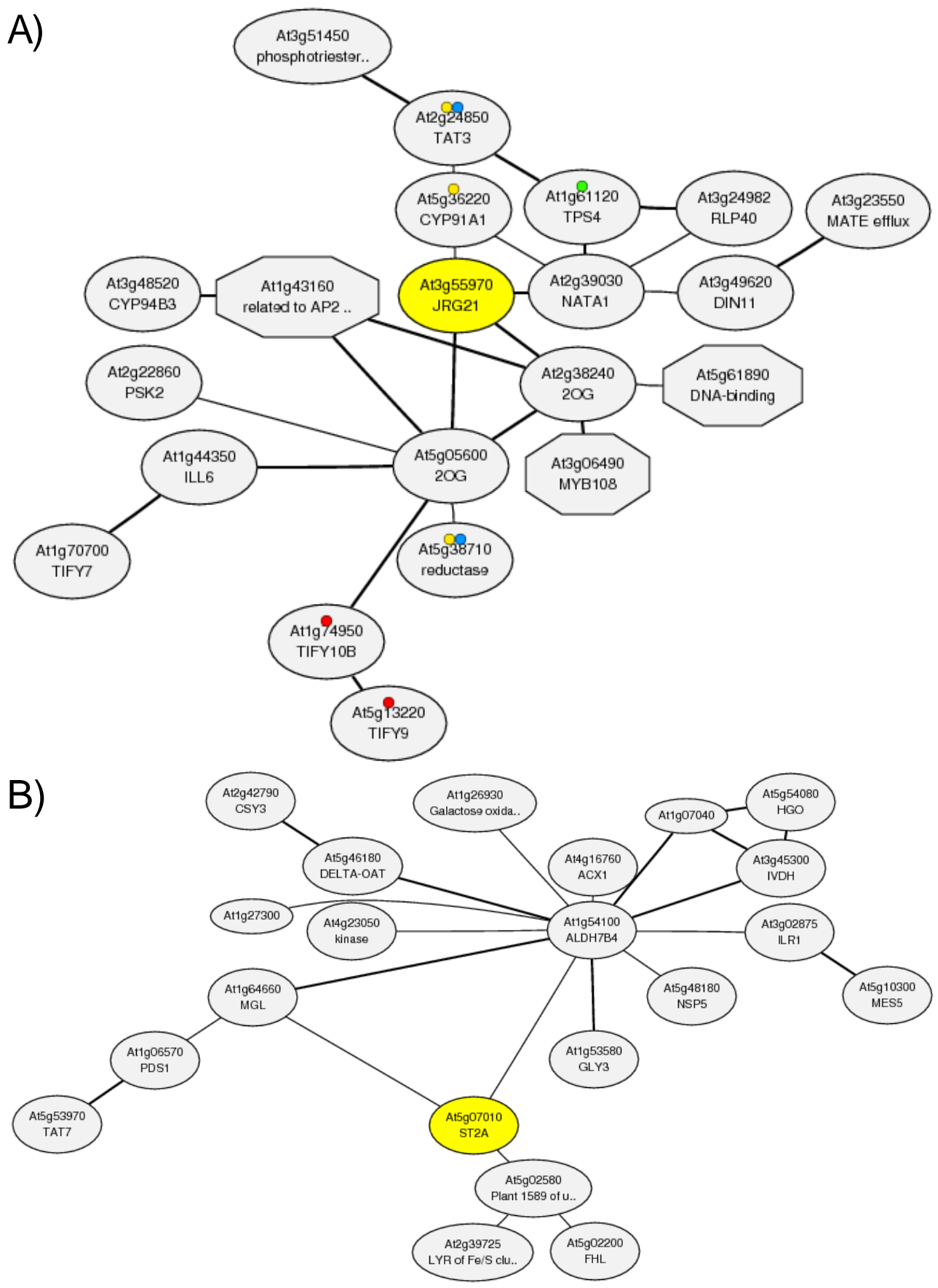

Supplemental figure 2: Search for glucosyltransferase genes products by co-expression analysis

Micro-array co-expression analysis of gene products involved in 12-hydroxy-JA metabolism. A) Coexpression gene network around Jasmonic acid oxidase 3 (JOX3, At3g55970, yellow circle). JOX3 is coexpressed with genes involved in plant hormone signal transduction (red marks), biosynthesis of secondary metabolites (yellow marks), diterpenoid biosynthesis (green marks), and biosynthesis of antibiotics (blue marks). B) Co-expression gene network of sulfotransferase 2a (ST2a, At5g07010, yellow circle). Octagonal shape indicates transcription factors and circular shape others. Networks are drawn based on Mutual Ranks (MR) giving an average correlation of two genes indicating stronger correlation by smaller values ( $M R=1-5$ : bold lines, $M R=5-30$ : normal lines, $M R>30$ : weak lines). Marks indicate a common KEGG pathway. Access 02.05.2018; the page was prepared on Dec. 14. 2017 for ATTED-II version 9.1 

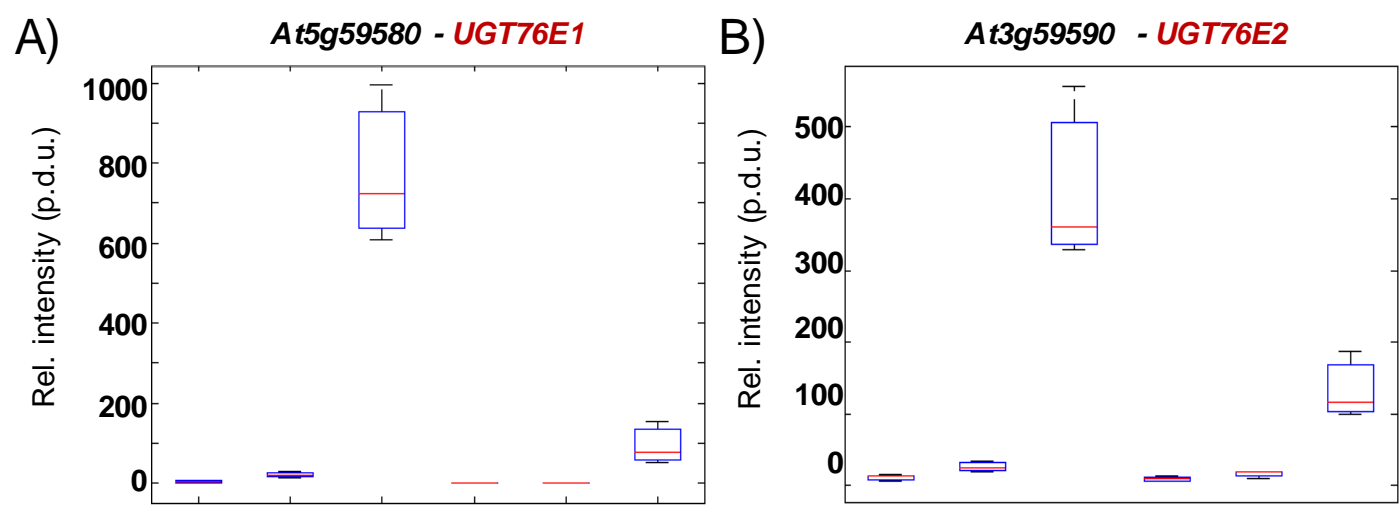

C)

At3g46670 - UGT76E11

D)

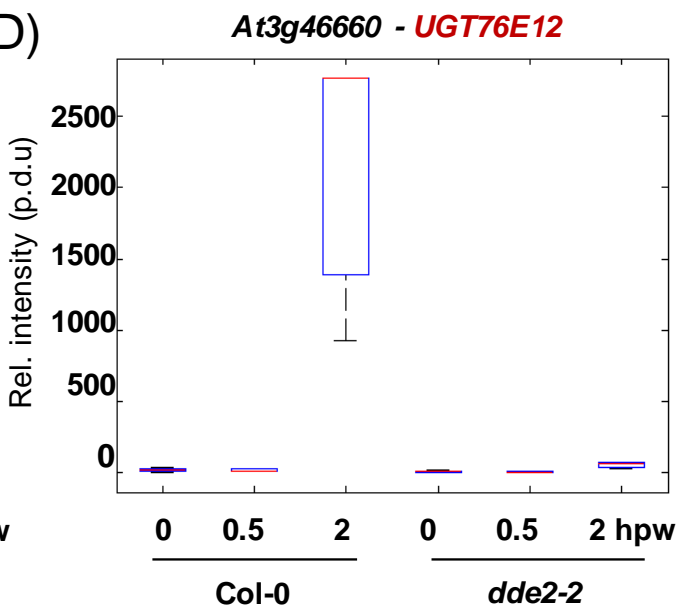

Supplemental figure 3: Expression of UGT76E1, UGT76E2, UGT76E11, and UGT76E12 in A. thaliana leaves after wounding

Transcriptomic analysis of Col-0 and a JA-deficient mutant (dde2-2). Plants were grown for six weeks at $22^{\circ} \mathrm{C}$ under short day conditions ( $8 \mathrm{~h}$ light $/ 16 \mathrm{~h}$ dark). Leaves were wounded three times across the mid vein by squeezing with forceps. Damaged rosette leaves were harvested at indicated time points (hours post wounding (hpw)). RNA was isolated and used for micro-array analysis. The transcriptomics data was recorded by a $44 \mathrm{k}$ Affimetrix micro-array analysis and the readout is given in relative intensities (procedure defined units (p.d.u.)). Given are Box-Whisker-plots of the relative intensities of A) At5g59580, UGT76E1, B) At5g59590, UGT76E2, C) At3g46670, UGT76E11, and D) At3g46660, UGT76E12. The data represents medians of three experiments. Data from Dr. A. Mosblech, Dr. K. Feussner and Prof. Dr. I. Heilmann, 2010 unpublished. 
A)

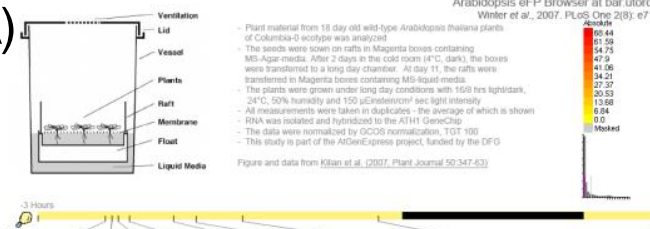

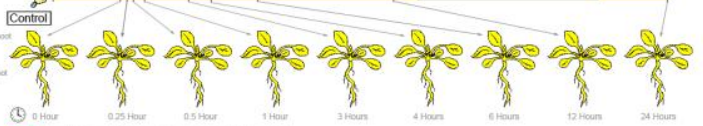

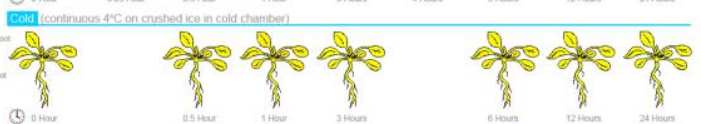

(3) 0 How

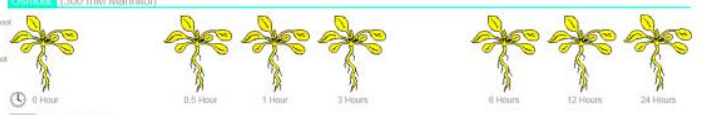

*

0.1

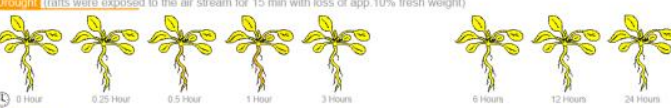
(-).

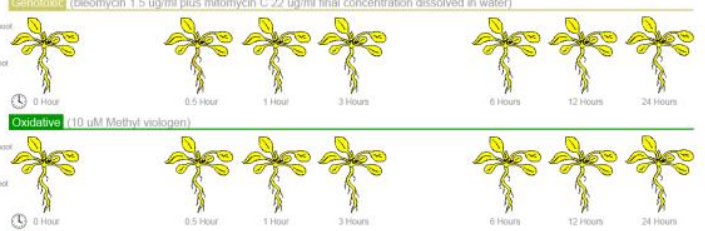

Tำt

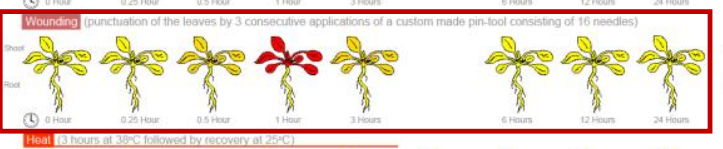

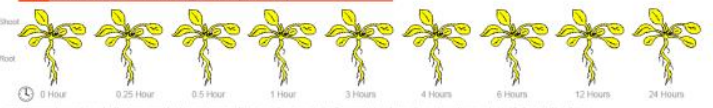
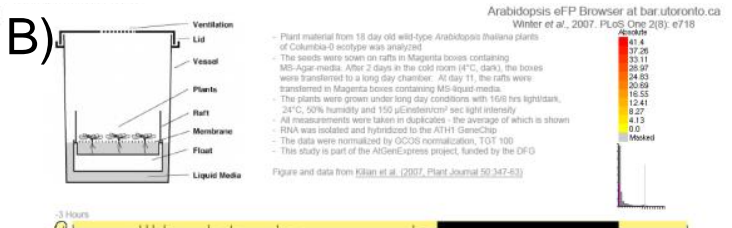

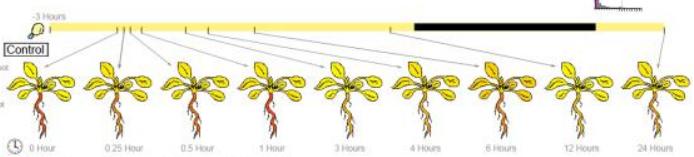

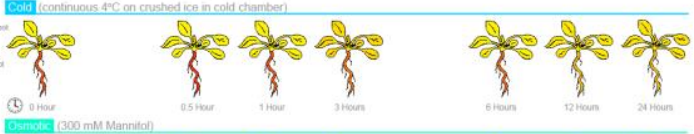

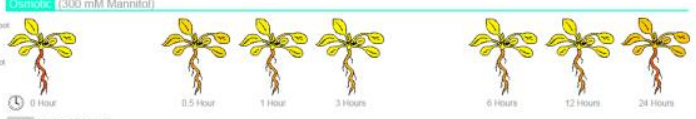

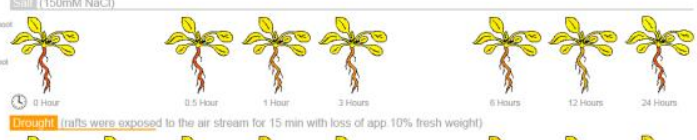

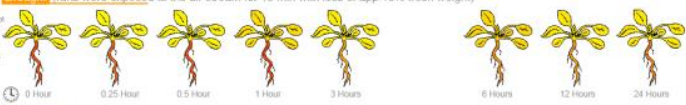

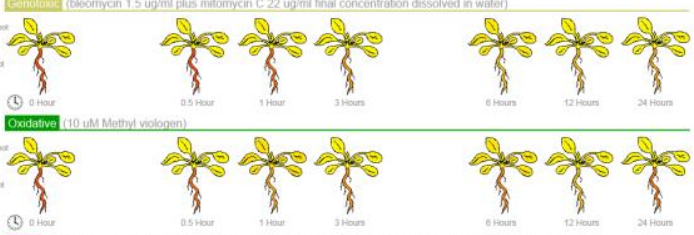

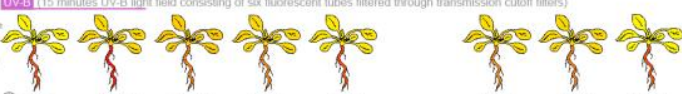

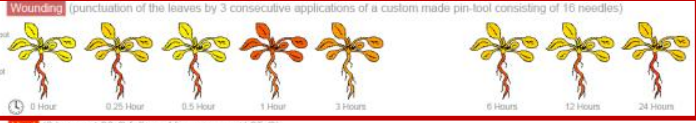

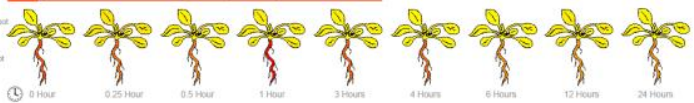

\section{Supplemental figure 4: Expression of $U G T 76 E 1$ and $U G T 7 E 2$ after abiotic stresses}

Expression analysis of indicated genes after abiotic stresses: cold, osmotic, alt, drought, genotoxic, oxidative, UV-B, wounding, and heat. Stress responses are illustrated from $0-24$ hours after stimulus A) Expression pattern of $U G T 76 E 1$. Response after wounding is highlighted (red box). B) Expression pattern of $U G T 76 E 2$. Response after wounding is highlighted (red box). The graphics are taken from Arabidopsis eFP-Browser; original data from Winter et al., 2007. Access 02.05.2018 


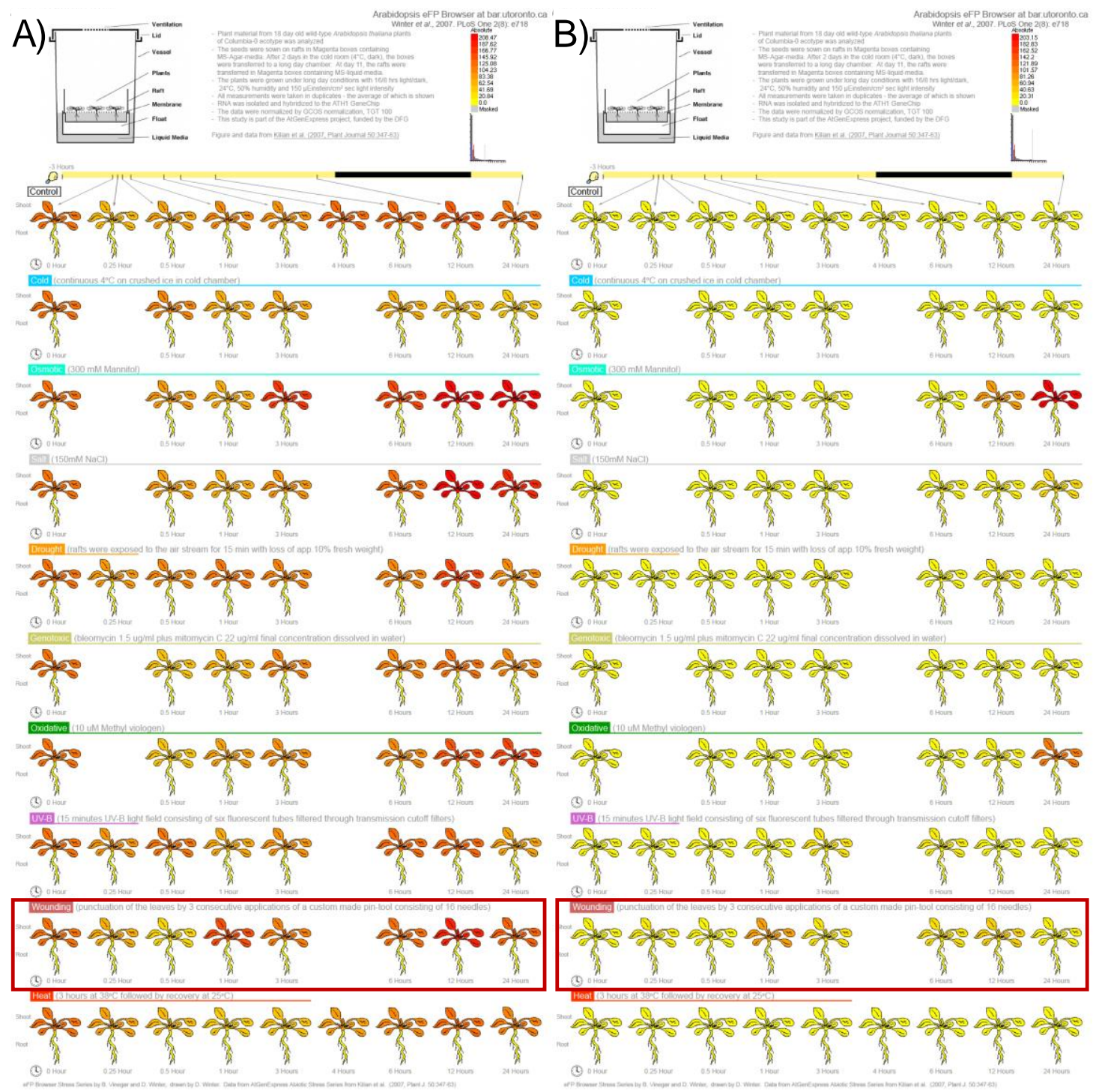

\section{Supplemental figure 5: Expression of UGT76E11 and UGT7E12 after abiotic stresses}

Expression analysis of indicated genes after abiotic stresses: cold, osmotic, alt, drought, genotoxic, oxidative, UV-B, wounding, and heat. Stress responses are illustrated from 0 -24 hours after stimulus A) Expression pattern of UGT76E11. Response after wounding is highlighted (red box). B) Expression pattern of UGT76E12. Response after wounding is highlighted (red box). The graphics are taken from Arabidopsis eFP-Browser; original data from Winter et al., 2007. Access 02.05.2018. 

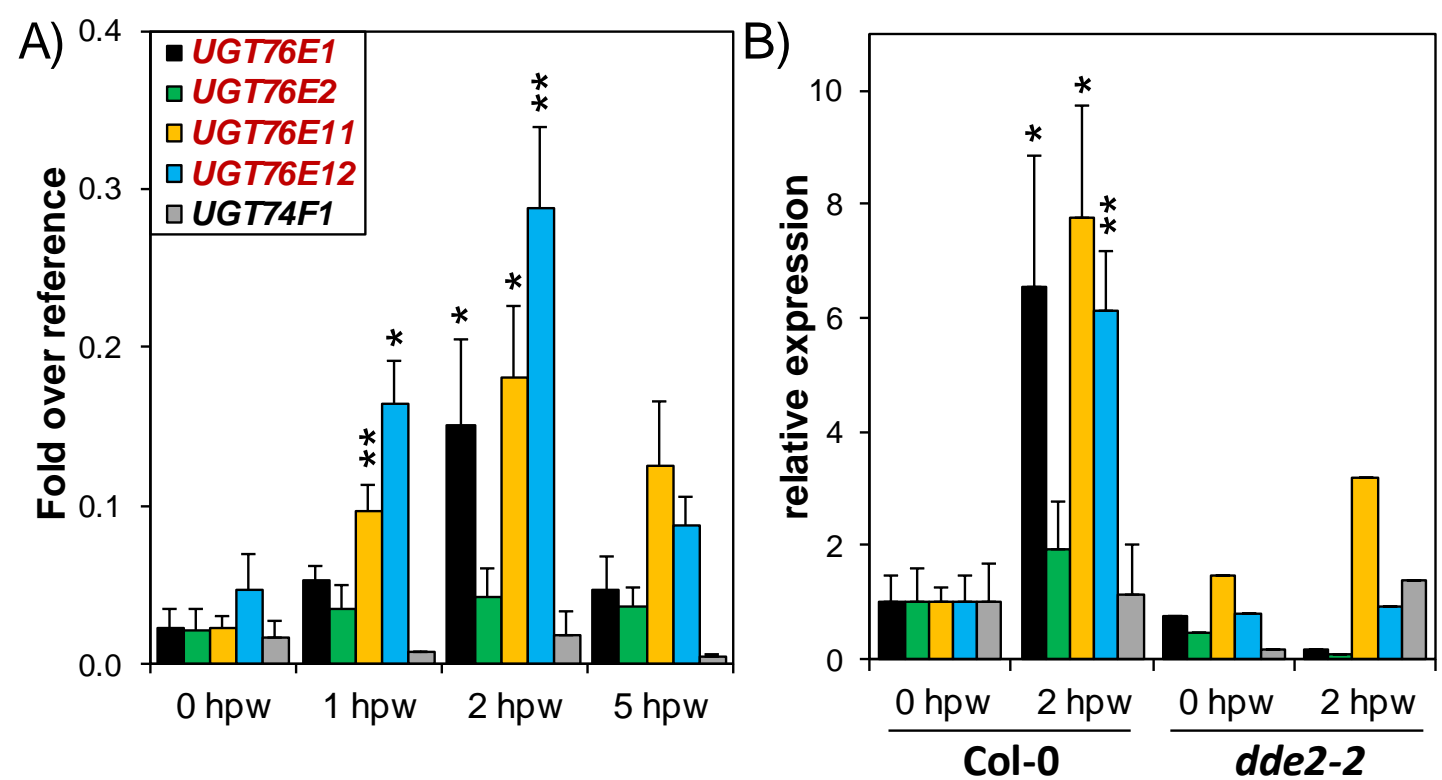

Supplemental figure 6: Expression of UGT76E1, UGT76E2, UGT76E11, UGT76E12, and UGT74F1 in Col-0 and JA-deficient mutant after wounding

Quantitative real-time PCR of UGT76E1 (red), UGT76E2 (green), UGT76E11 (orange), UGT76E12 (blue), and $U G T 74 F 1$ (grey). Col-0 and JA-deficient (dde2-2) plants were grown for six weeks at $22{ }^{\circ} \mathrm{C}$ under short day conditions ( $8 \mathrm{~h}$ light/16 h dark). Leaves were wounded three times across the mid vein by squeezing with forceps. Damaged rosette leaves were harvested at indicated time points (hours post wounding (hpw)), RNA was isolated, and appr. $1 \mu \mathrm{g}$ transcribed complementary DNA were used for PCR. All expression values are normalized to actin 8 as reference. Relative expression of the transcripts normalized to their respective expression levels at $0 \mathrm{hpw}$ in Col-0 and $d d e 2.2$. Col-0-data represents the mean value $+\mathrm{SD}$ of three biological replicates, dde2.2-data presents one experiment. 10 plants per time point were pooled for one replicate. Asterisks indicate significance by one-sided T-Test with ${ }^{*} \mathrm{p}<0.05$, $* * p<.0 .01$. 


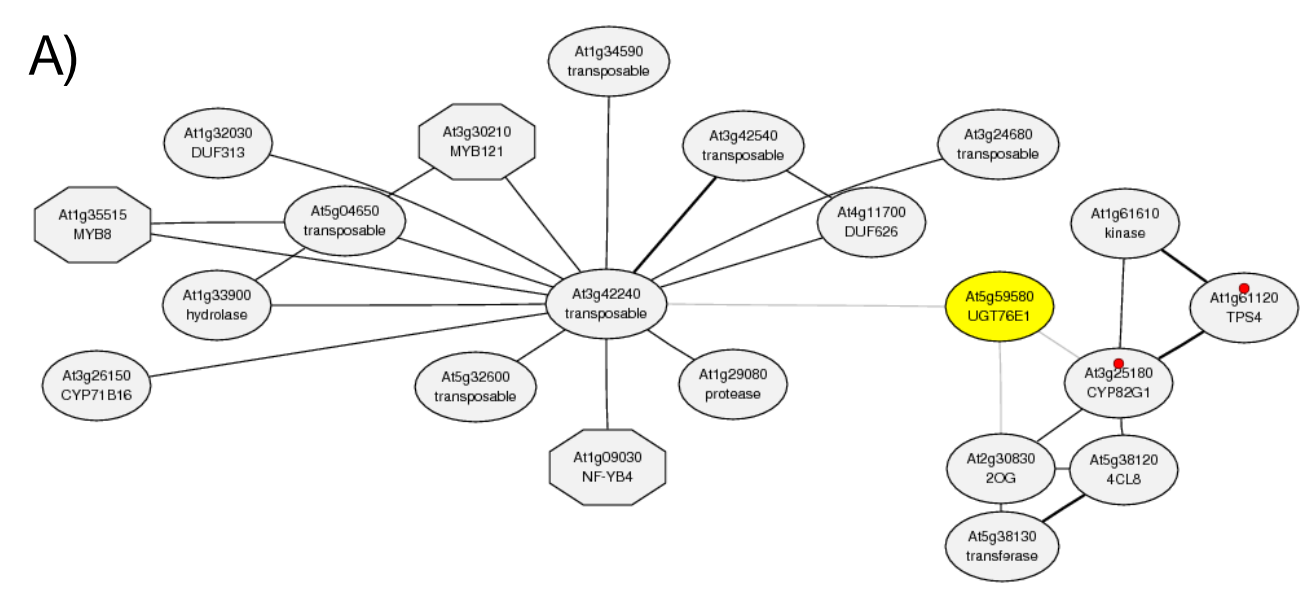

B)

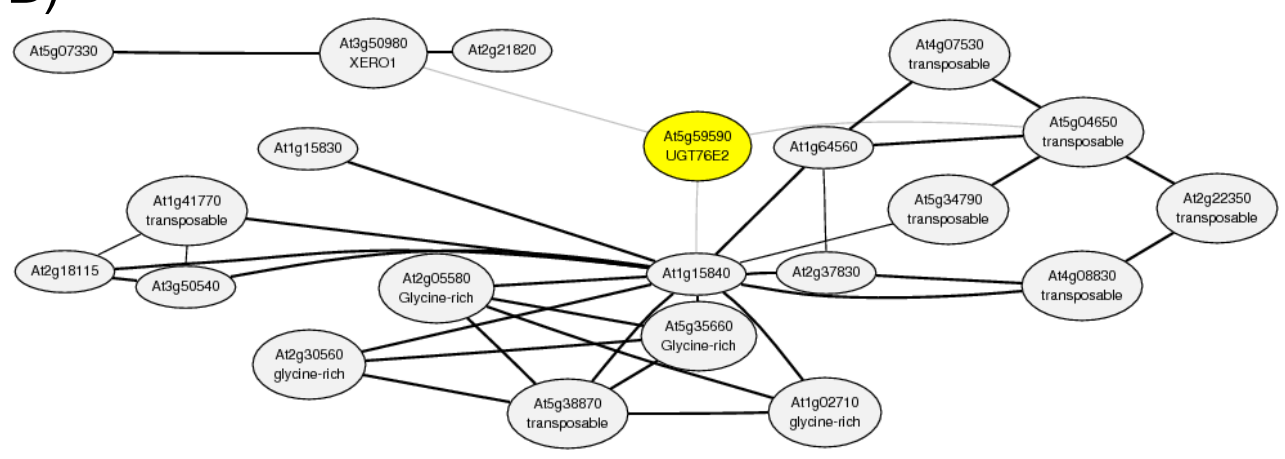

\section{Supplemental figure 7: Co-expression analysis of $U G T 76 E 1$ and $U G T 76 E 2$}

Micro-array co-expression analysis of gene products of indicated genes. A) Co-expression gene network around UGT76E1 (At5g59580, yellow circle). UGT76E1 is co-expressed with genes involved in diterpenoid biosynthesis (red marks). B) Co-expression gene network of UGT76E2 (At5g59590, yellow circle). Octagonal shape indicates transcription factors and circular shape others. Networks are drawn based on Mutual Ranks (MR) giving an average correlation of two genes indicating stronger correlation by smaller values ( $M R=1-5$ : bold lines, $M R=5-30$ : normal lines, $M R>30$ : weak lines). Marks indicate a common KEGG pathway. Access 02.05.2018; the page was prepared on Dec. 14. 2017 for ATTED-II version 9.1 


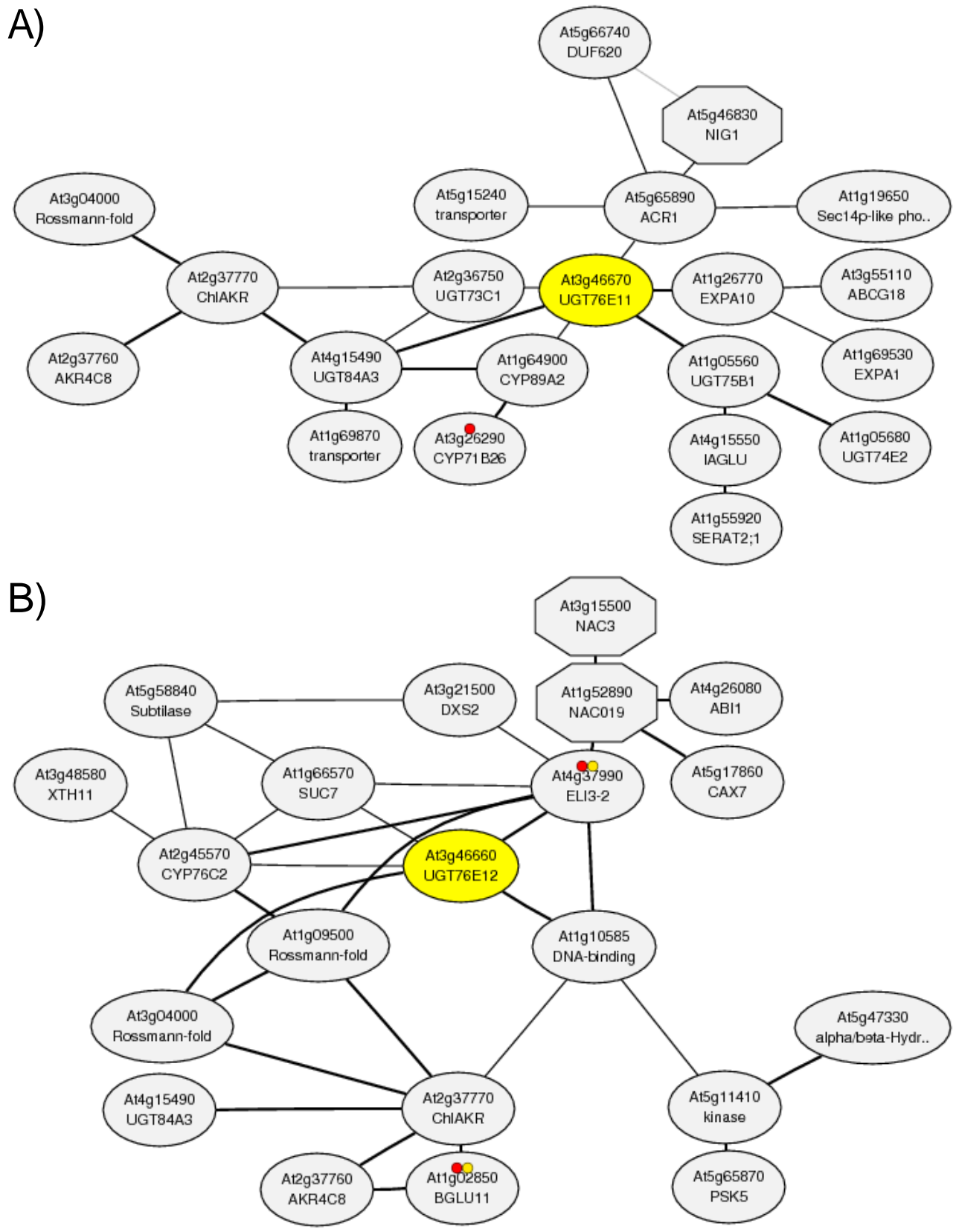

Supplemental figure 8: Co-expression analysis of UGT76E11 and UGT76E12

Micro-array co-expression analysis of gene products of indicated genes. A) Co-expression gene network around UGT76E11 (At3g46670, yellow circle). UGT76E11 is co-expressed with genes involved in biosynthesis of secondary metabolites (red marks). B) Co-expression gene network of UGT76E12 (At3g46660, yellow circle). UGT76E12 is co-expressed with genes involved in biosynthesis of secondary metabolites (red marks) and genes of the phenylpropanoid biosynthesis (yellow marks). Octagonal shape indicates transcription factors and circular shape others. Networks are drawn based on Mutual Ranks (MR) giving an average correlation of two genes indicating stronger correlation by smaller values $(\mathrm{MR}=$ 1-5: bold lines, $\mathrm{MR}=$ 5-30: normal lines, $\mathrm{MR}>$ 30: weak lines). Marks indicate a common KEGG pathway. Access 02.05.2018; the page was prepared on Dec. 14. 2017 for ATTED-II version 9.1 
Supplemental table 1: Calculated protein parameters and predicted localizations for UGT76E1, UGT76E2, UGT76E11, UGT76E12 and UGT74F1

Information about the proteins were calculated from their primary amino acid sequences with different online-tools. The table depicts the molecular weight (MW), the specific extinction coefficient at $280 \mathrm{~nm}$ $(\varepsilon)$, the theoretical isoelectric point (pI), the presents of a signal peptide (SignalP), target peptides (TargetP), and the number of transmembrane domains (TMHMM) for UGT76E1, UGT76E2, UGT76E11, UGT76E12, and UGT74F1. The protein parameters were calculated with ProtParam and localization predictions were done with SignalP, TargetP (plant settings), and TMHMM online tools. Access was 04.05.2018.

\begin{tabular}{c|ccccc}
\hline Parameter & UGT76E1 & UGT76E2 & UGT76E11 & UGT76E12 & UGT74F1 \\
\hline $\mathrm{MW}(\mathrm{kDa})$ & 50.8 & 50.1 & 50.6 & 51.7 & 50.3 \\
$\varepsilon\left(\mathrm{kM}^{-1} \mathrm{~cm}^{-1}\right)$ & 66.4 & 63.5 & 58.0 & 55.5 & 62.4 \\
pl & 6.6 & 5.4 & 5.9 & 6.0 & 5.5 \\
SignalP & $/$ & $/$ & $/$ & $/$ & $/$ \\
TargetP & mitochondrial & $/$ & $/$ & $/$ & $/$ \\
TMHMM & 0 & 0 & 0 & 0 & 0 \\
(no.) & & & & & \\
\hline
\end{tabular}

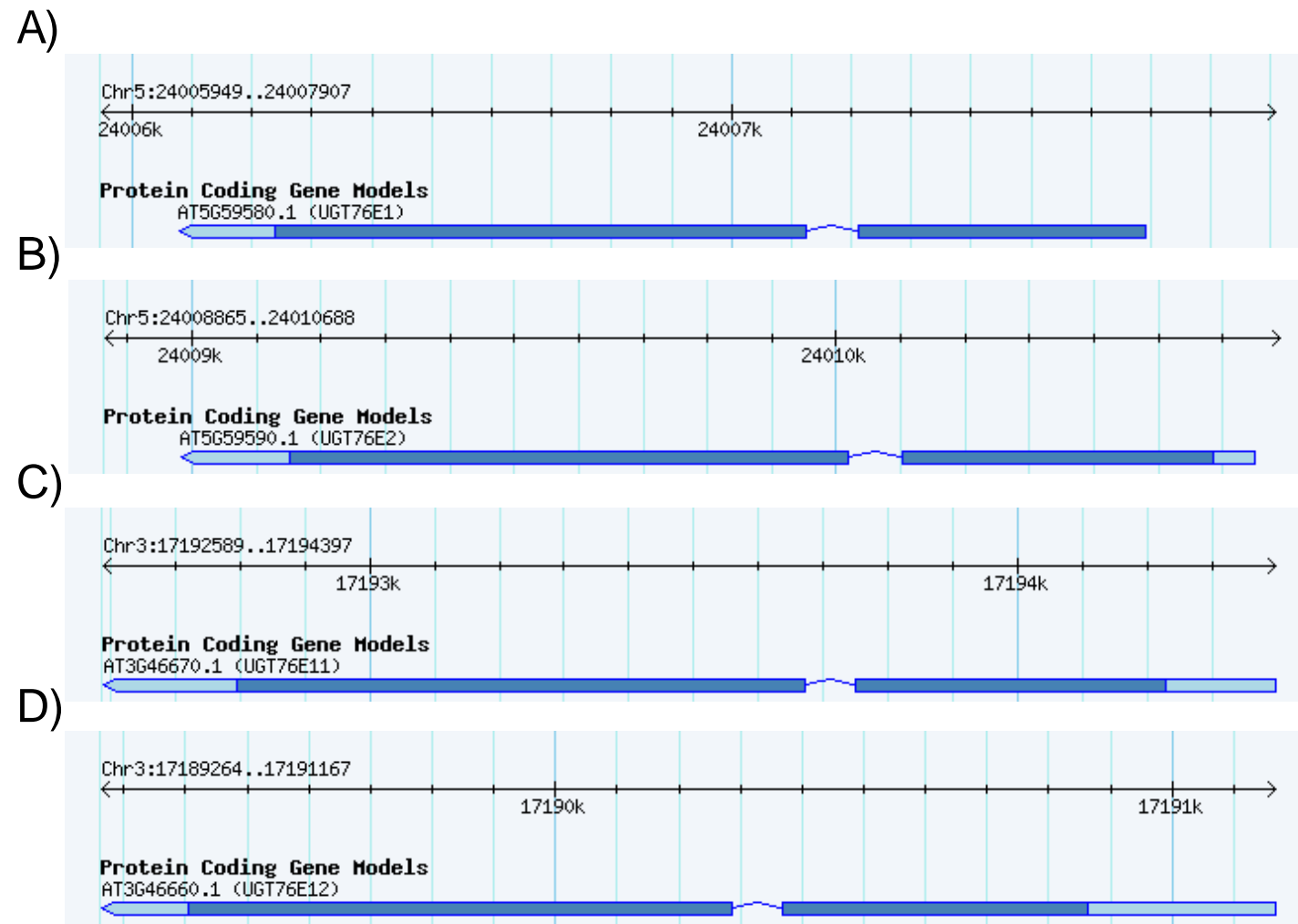

Supplemental figure 9: Gene structures of UGT76E1, UGT76E2, UGT76E11, and UGT76E12 in A. thaliana genome

Gene maps of the indicated UGTs in the A. thaliana genome. Nucleotide bar depicts the location in the chromosome (black bar). Gene structure is shows in coding region (dark blue), untranslated region (light blue), and introns (arrow up lines). Shown are gene maps of A) UGT76E1, B) UGT76E2, C) UGT76E11, and D) $U G T 76 E 12$. Graphics taken from The Arabidopsis Information Resource (TAIR), access 02.05.2018. 

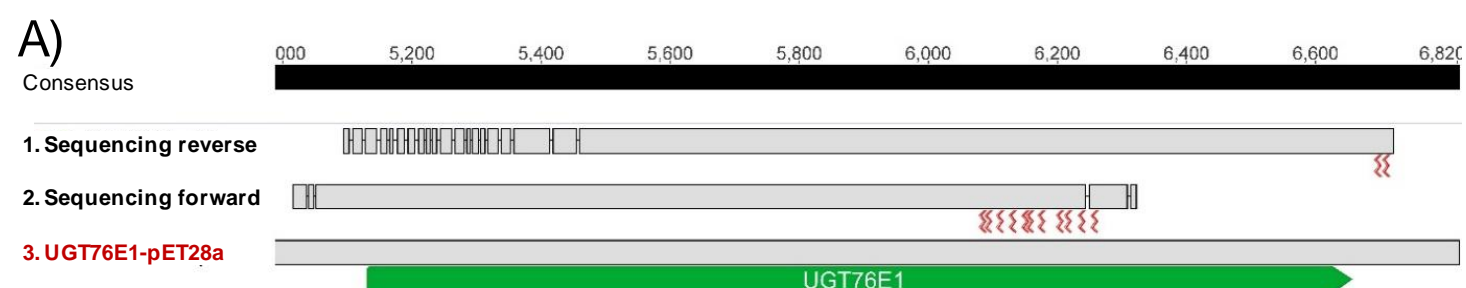

B) Consensus
1.Sequencing forward 2. Sequencing reverse 3. UGT76E2-pET28a

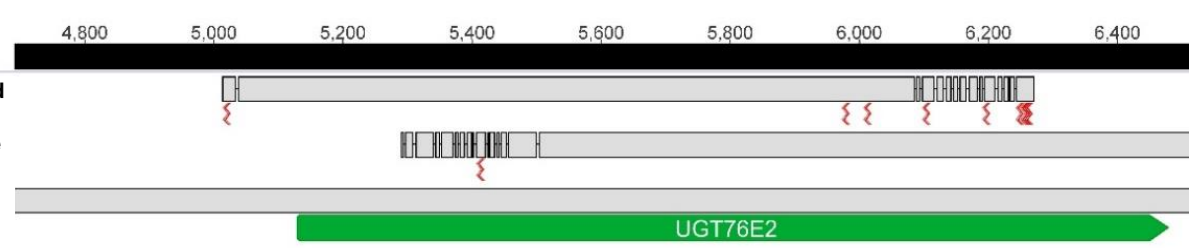

C)

Consensus

1. Sequencing forward 2. Sequencing reverse 3. UGT76E11-pET28a

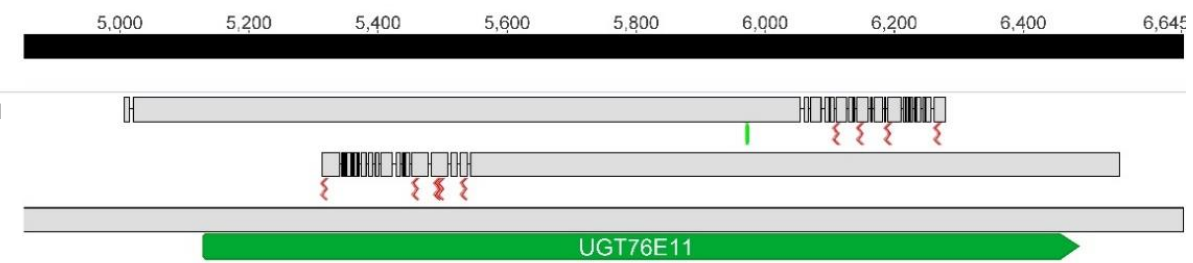

D) Consensus 2. Sequencing forward 3. UGT76E12-pET28a

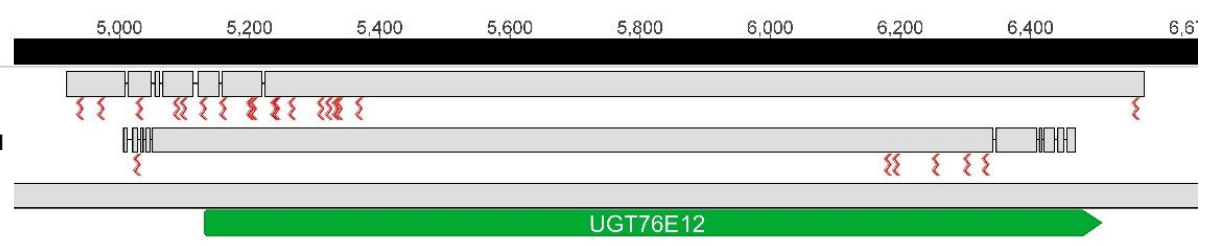

E) Consensus 1. Sequencing reverse 2. Sequencing forward 3. UGT76E12-pET28a

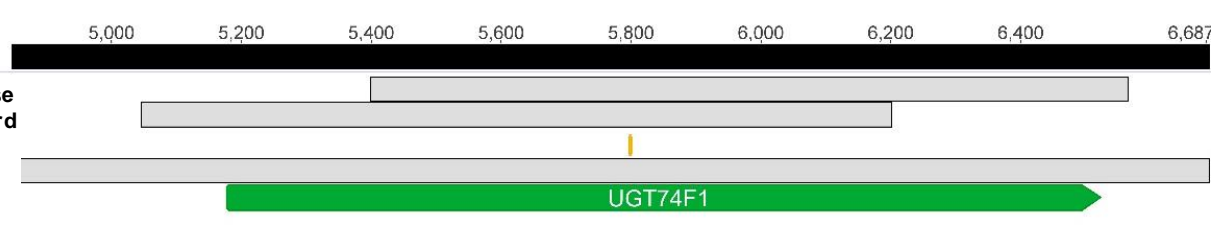

Supplemental figure 10: Sequencing results of UGT76E1, UGT76E2, UGT76E11, UGT76E12, and UGT74F1 integrated into the expression vector

The coding sequences of indicated genes were amplified from complementary DNA (cDNA) 2 hours post wounding. The sequence of UGT74F1 was amplified from root cDNA. Indicated UGT-sequences (green annotations) were fused to an N-terminal His-tags of pET28a vectors for heterologous protein expression in E. coli. Correct cloning was checked by sequencing from two directions (forwards and reverse). Poor sequencing results are marked with red tildes, manual insertion is given as green line, and differing sequence is marked with yellow line. Complete and correct sequencing results are shown for A) UGT76E1, B) UGT76E2, C) UGT76E11, and D) UGT76E12. E) The sequence of UGT74F1 illustrates a splice variant of the template. All alignments were done with the Geneious algorithm as global alignment with free end gaps and $65 \%$ cost matrix in Geneious version 8.1 (Biomatters). Available from http://www.geneious.com 
A)

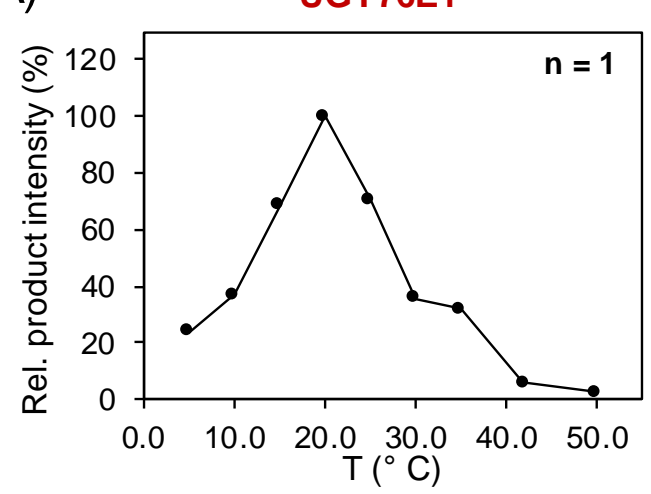

C)

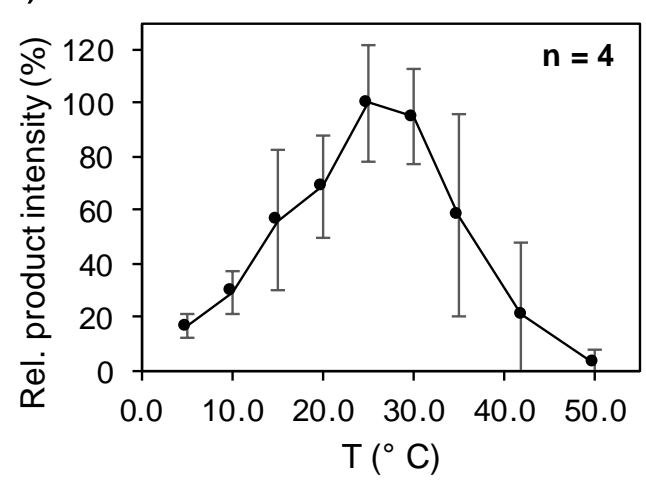

B)

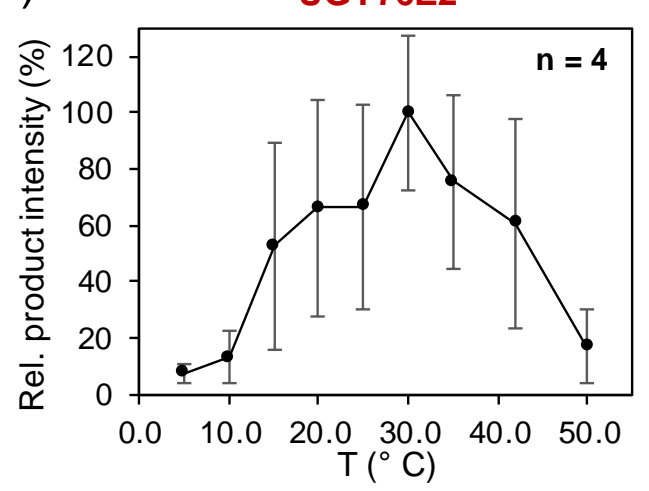

D)

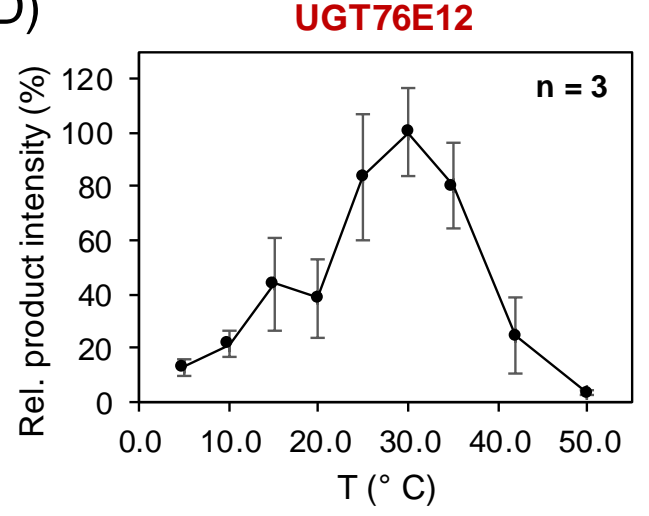

Supplemental figure 11: Optimum temperature of UGT7E1, UGT76E2, UGT76E11, and UGT7E12

Temperature optima for the indicated UGTs were determined in the range of 4 to $50^{\circ} \mathrm{C} .0 .1 \mathrm{mM} \omega$ hydroxy-hexadecanoic acid and $0.5 \mathrm{mM}$ UDP-Glc in the gel filtration buffer of the receptive enzyme were equilibrated at the indicated temperatures for $1 \mathrm{~h}$. The product formation was detected by LC-MS and shown as relative signal intensities (\%) over temperature for A) UGT76E1, B) UGT76E2, C)

UGT76E11, and D) UGT76E12. Data represents one experiment for UGT76E1. All other measurements are means with $\pm \mathrm{SD}$ of and at least three independent experiments 


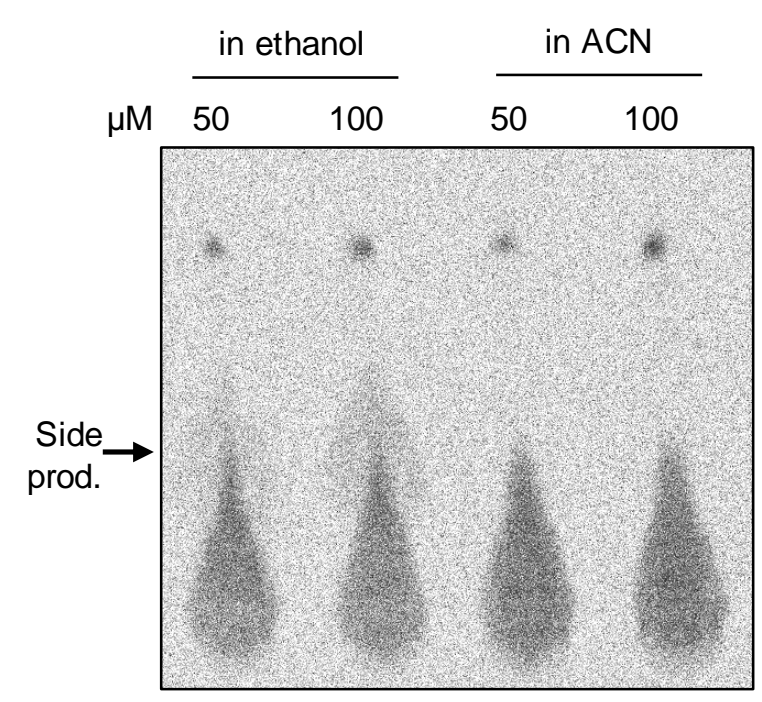

\section{Supplemental figure 12: UGT76E12 shows side activity towards ethanol}

The substrate specificities of UGT76E12 was analyzed by an activity assay with uniformly labeled UDP${ }^{14} \mathrm{C}(\mathrm{U})$-Glc towards $\omega$-hydroxy-hexadecanoic acid. The assay was performed with $5 \mu \mathrm{g}$ homogeneous UGT76E12, $50 \mu \mathrm{M}$ or $100 \mu \mathrm{M} \omega$-hydroxy-hexadecanoic acid dissolved in ethanol or acetonitrile (ACN) and $30 \mu \mathrm{M}$ UDP-Glc, $0.02 \mu \mathrm{Ci}$ UDP- ${ }^{14} \mathrm{C}(\mathrm{U})-\mathrm{Glc}$ in the buffer $(20 \mathrm{mM}$ tris/ $\mathrm{HCl}, \mathrm{pH} 7.5,50 \mathrm{mM} \mathrm{NaCl})$. The reaction took place for $1 \mathrm{~h}$ at $25^{\circ} \mathrm{C}$. After metabolite extraction and thin layer chromatography (TLC), the TLC-plates were incubated on phosphoscreens for 5 days and the radioactive signals detected. The arrow indicates a side product (side-prod.) with ethanol. The data represents one experiment.

\section{Supplemental table 2: Chemical structures of the UGT-substances}

Chemical structures of hexadecanoic acid (16:0), $\omega$-hydroxy-16:0 ( $\omega$-OH-16:0), 2-hydroxy-16:0 (2-OH16:0), 3-hydroxy-16:0 (3-OH-16:0), hexadecanol (OH-C16), benzoic acid (BA), salicylic acid (SA), pipecolic acid (Pip), indole-3-caboxylic acid (ICA), abscisic acid (ABA), zeatin, gibberellic acid (GA), 12- 12-oxo-phytodienoic acid (12-OPDA), JA, 12-hydroxy-JA (12-OH-JA), 11-hydroxy-JA (11-OH-JA), 12-hydroxy-JA-methyl ester (12-OH-JA-ME), quercetin, dihydro-myricetin (dh-myricetin), and dihydrokaempferol (dh-kaempferol).

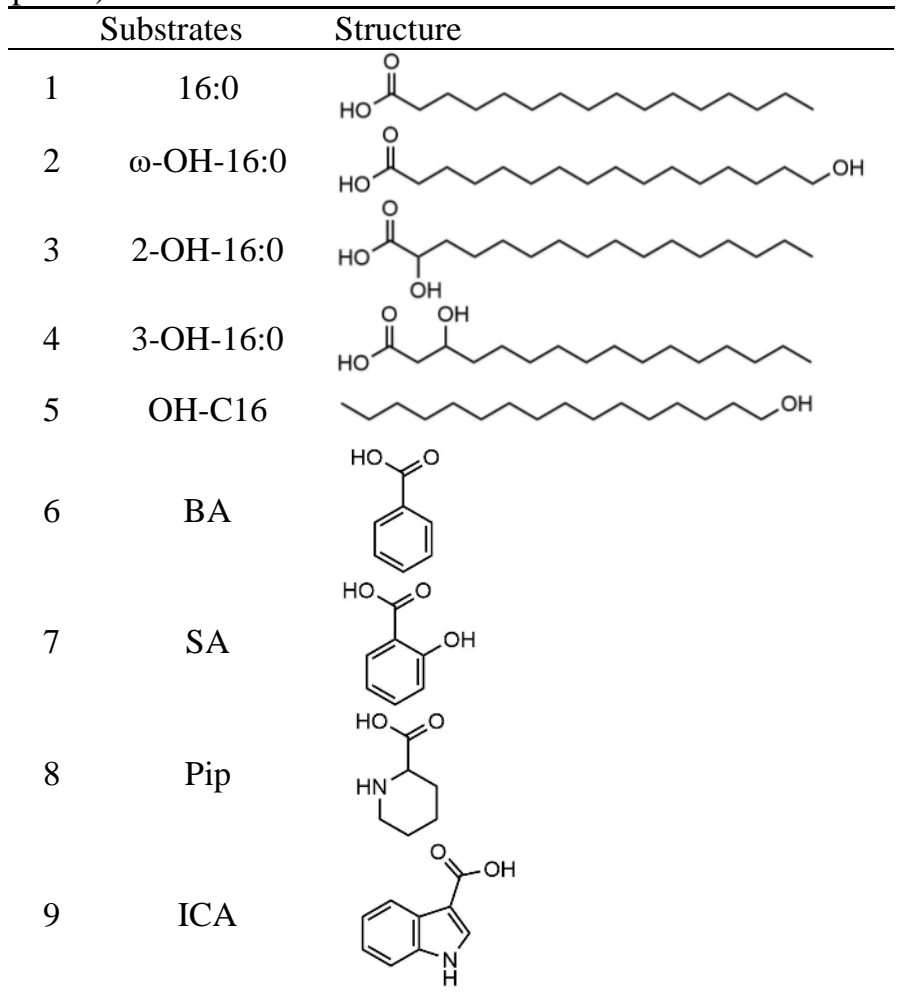




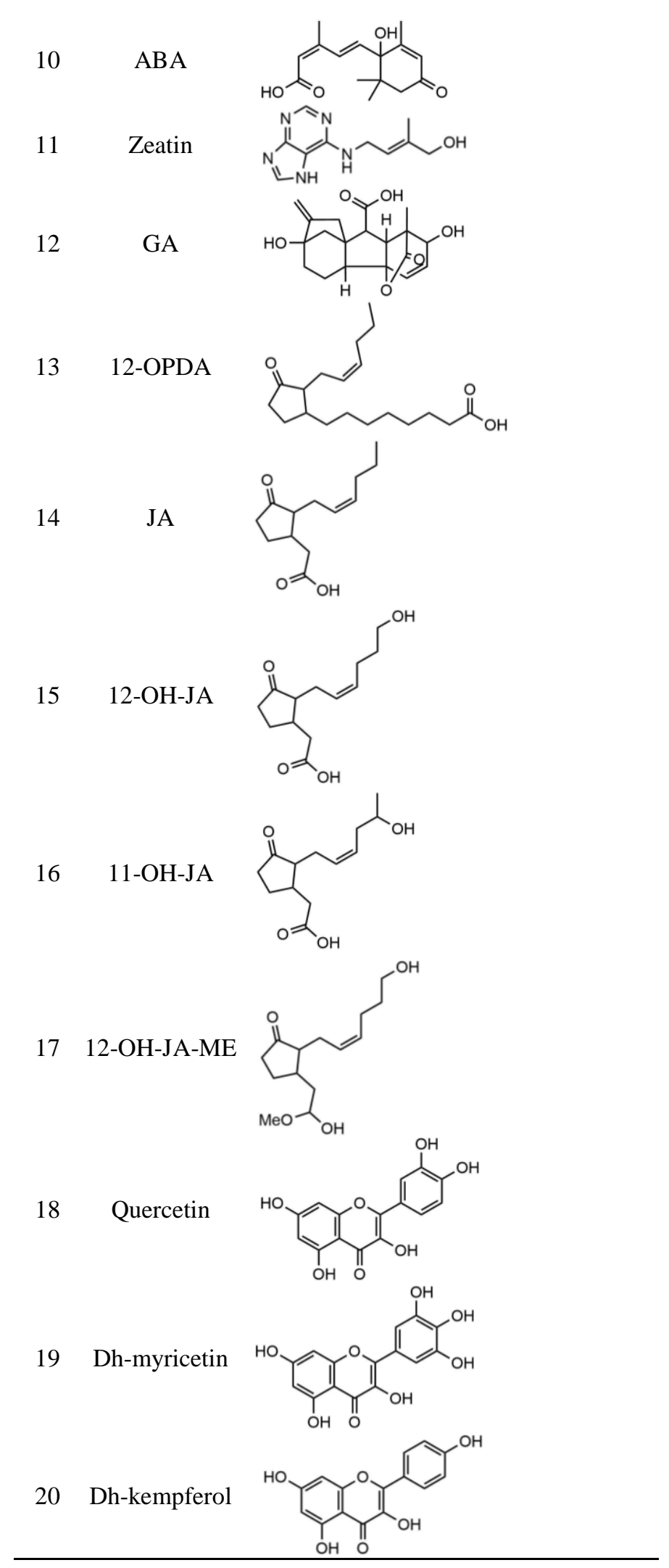



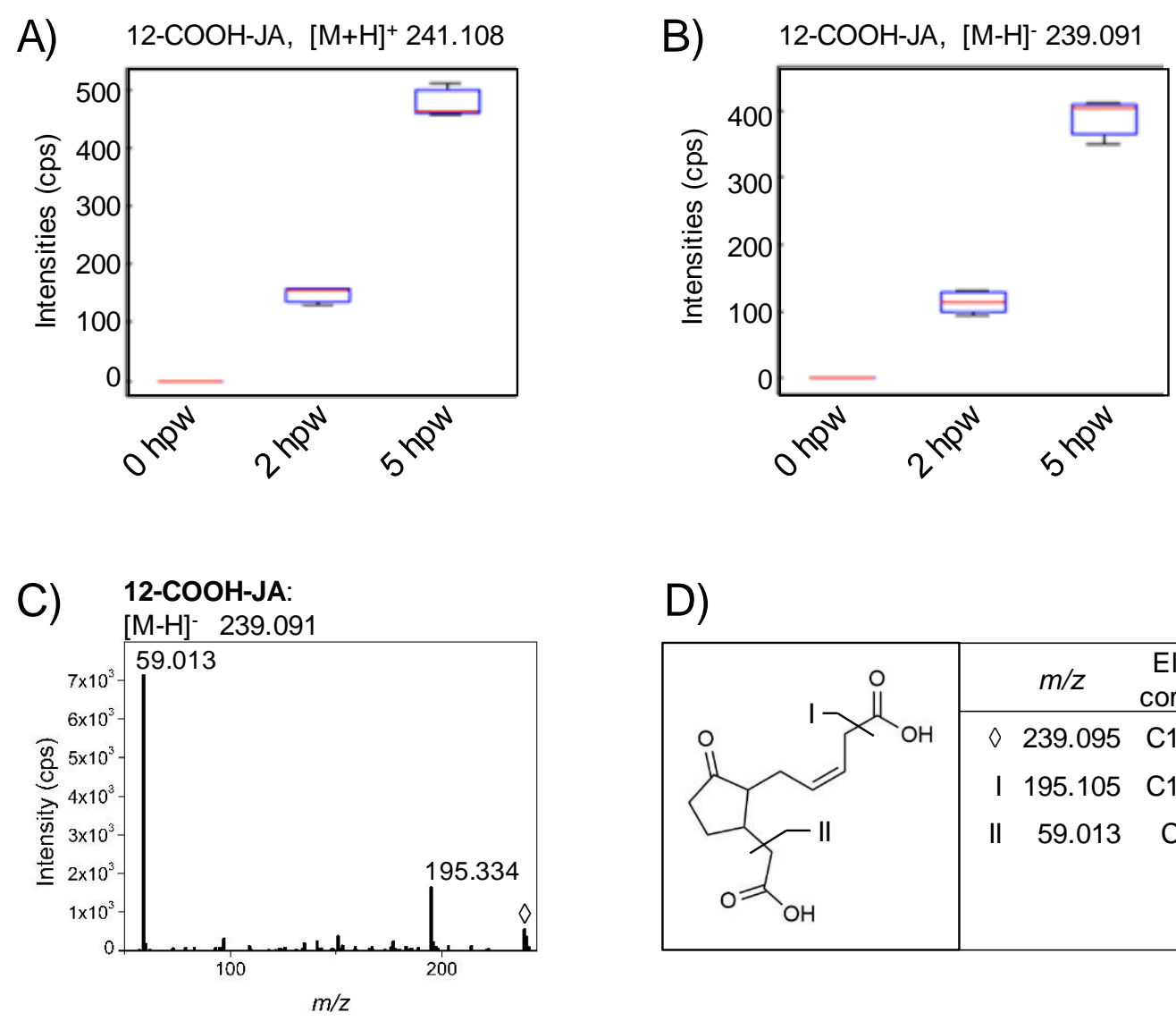

D)

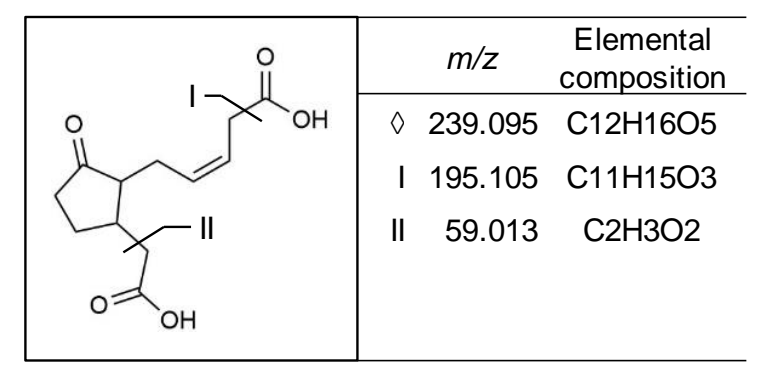

Supplemental figure 13: 12-carboxy-JA was identified in leaves after wounding

The metabolite 12-carboxy-JA (12-COOH-JA) was identified in the ex vivo metabolite fingerprinting analysis. Therefore, plants were grown for six weeks at $22^{\circ} \mathrm{C}$ under short day conditions $(8 \mathrm{~h} \mathrm{light} / 16 \mathrm{~h}$ dark). Leaves were wounded three times across the mid vein by squeezing with forceps. Damaged rosette leaves were harvested at 0,2 , and 5 hours post wounding (hpw), extracted, and used as substrate mix for the ex vivo activity assay. The extracts were resolved in $50 \mathrm{mM}$ Tris $\mathrm{pH} 8,100 \mathrm{mM} \mathrm{NaCl}$ buffer and $20 \mu \mathrm{l}$ acetonitrile. This samples were analyzed by mass spectrometry with a method, which was developed for non-targeted fingerprinting. Out of 1996 metabolite features with a $\mathrm{pVal}<10^{-6}$ the particular features of 12- $\mathrm{COOH}-\mathrm{JA}$ were found in the $\mathbf{A}$ ) positive ionization mode and $\mathbf{B}$ ) in the negative ionization mode shown as Box-Whisker-plots. The data represents three measurements of one experiments. 10 plants were pooled for each time point of one replicate. Data was analyzed with the MarVis tool (Kaever, 2014). C) The compound with the mass-to-charge ratio $(\mathrm{m} / \mathrm{z})$ 239.091( () was fragmented with a collision energy of $12 \mathrm{eV}$. D) Structure analyses of the main fragments can be assigned to 12-COOH-JA. Analysis was performed by Dr. Kirstin Feussner. Intensities given as counts per second (cps) 


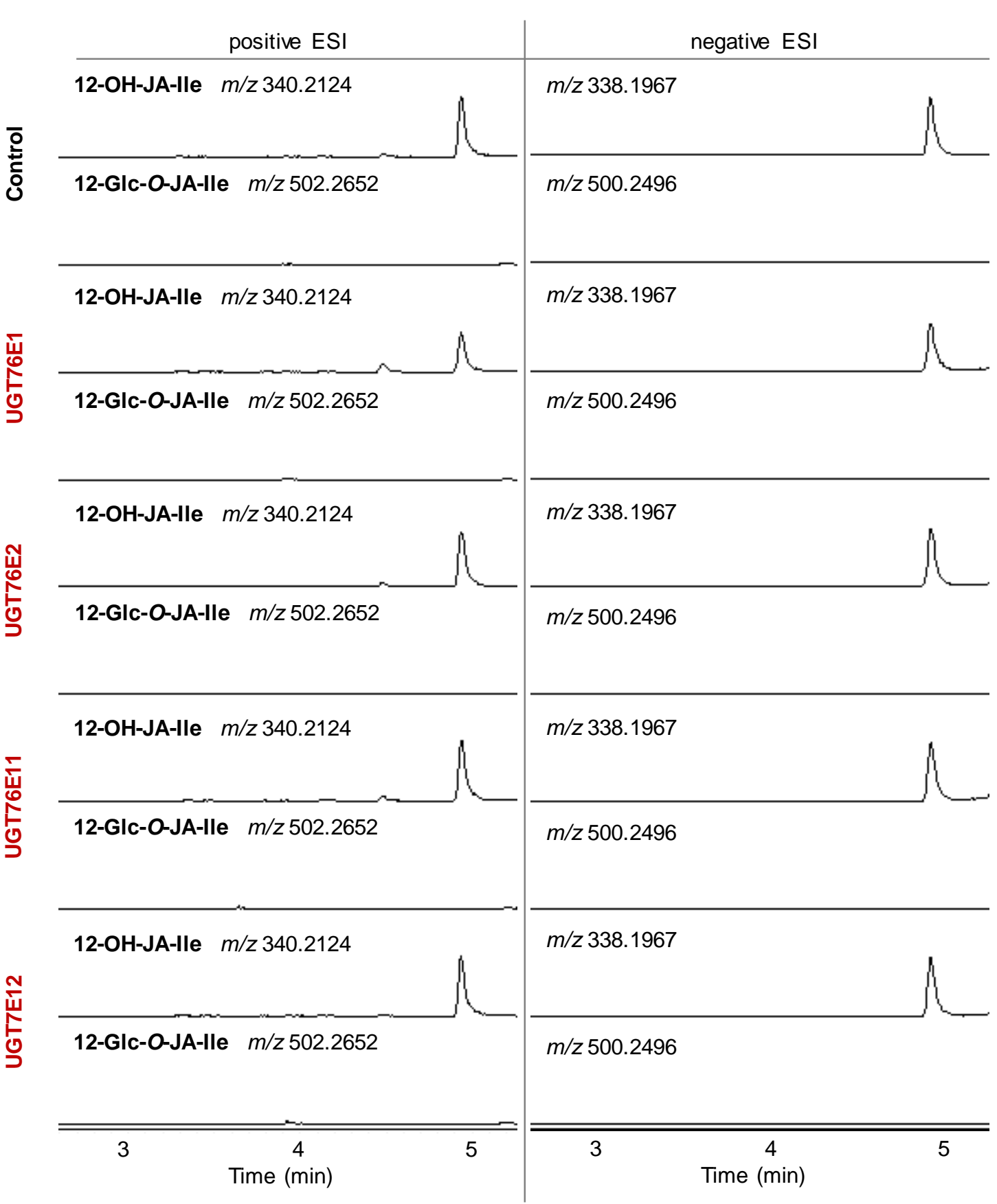

Supplemental figure 14: 12-O -glucosyl-jasmonoyl-isoleucine is not detected in plants

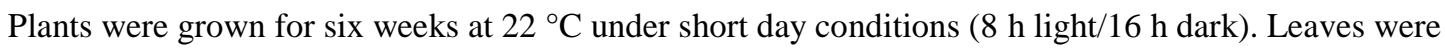
wounded three times across the mid vein by squeezing with forceps. Damaged rosette leaves were harvested at 5 hours post wounding (pool of 10 plants per sample), extracted, and used as substrate mix for the ex vivo activity assay. The extracts were resolved in $50 \mathrm{mM}$ Tris $\mathrm{pH} 8,100 \mathrm{mM} \mathrm{NaCl}$ buffer and the assay was performed with $0.1 \mathrm{mM}$ UDP-Glc and $100 \mu \mathrm{g}$ of the indicated active UGT-enzymes or inactive enzyme (control) for $1 \mathrm{~h}$ at $25{ }^{\circ} \mathrm{C}$. The reactions were stopped by adding acetonitrile and analyzed by mass spectrometry with a method, which was developed for non-targeted fingerprinting $\left(1996\right.$ features with $\left.\mathrm{pVal}<10^{-6}\right)$. Given are the extracted ion chromatograms of 12-hydroxy-jasmonoylisoleucine (12-OH-JA-Ile) in the negative ionization mode (mass-to-charge ration $(\mathrm{m} / \mathrm{z}) 338.1967)$ and in the positive ionization mode $(\mathrm{m} / \mathrm{z}, 340.2124)$ as well as for $12-O$-glucosyl-jasmonoyl-isoleucine $(12-O$ Glc-JA-Ile) in the positive ionization mode $(\mathrm{m} / \mathrm{z} 502.2652)$, and the negative ionization $(\mathrm{m} / \mathrm{z} 500.2496)$. All chromatograms show relative signal intensities fixed to $10^{4}$ counts per second over the time. The data is representative for three measurements of one experiments. Samples were measured by Dr. Kirstin Feussner. Data was analyzed with the MarVis tool (Kaever, 2014). 


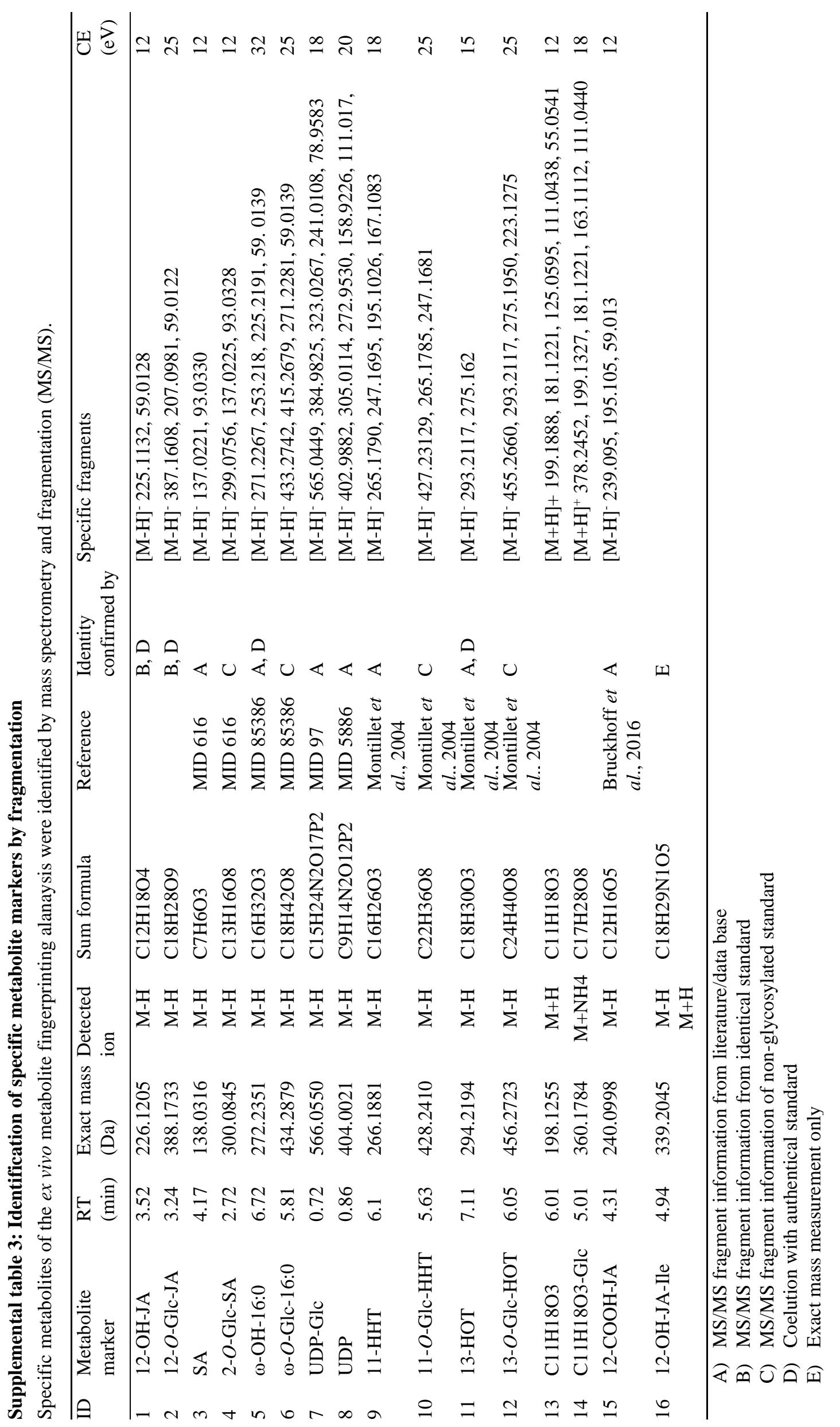




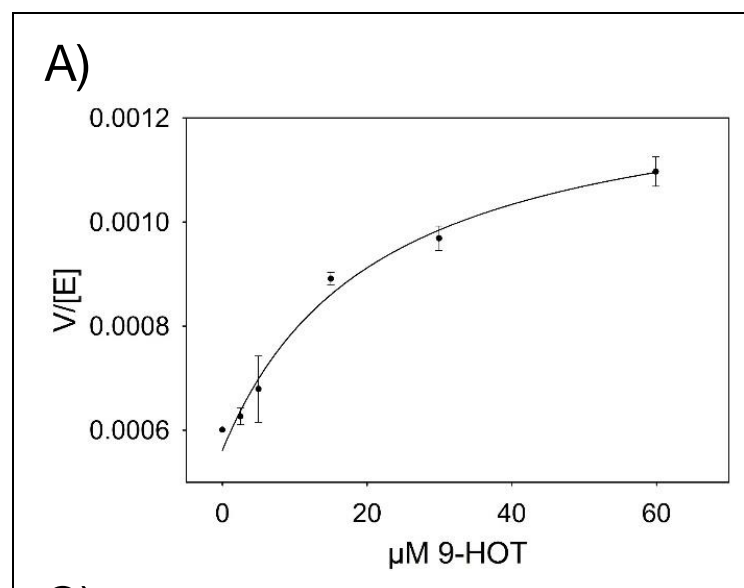

C)

B)
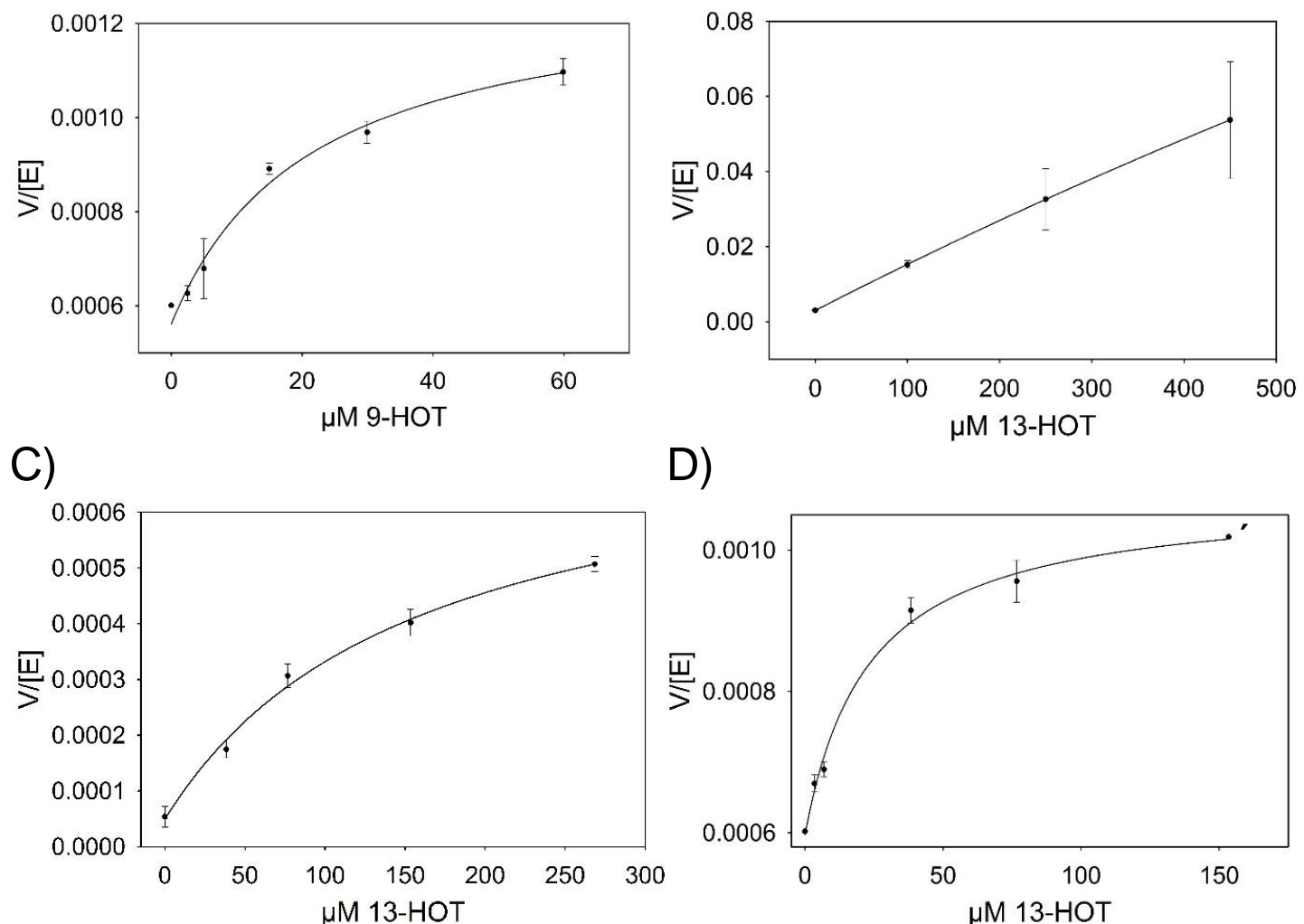

D)

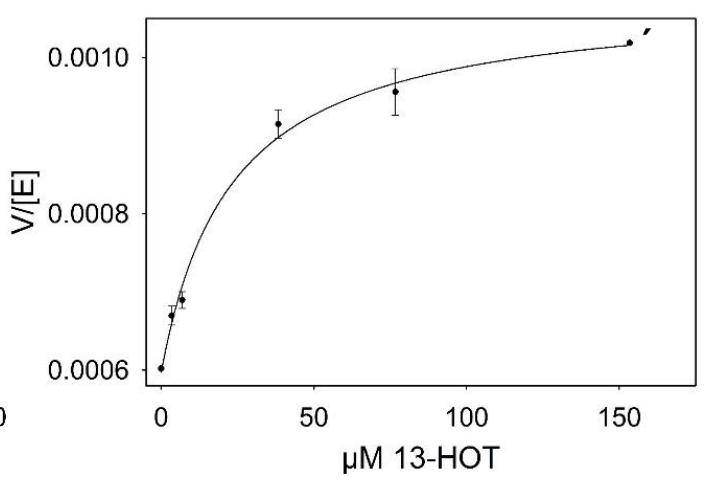

E)

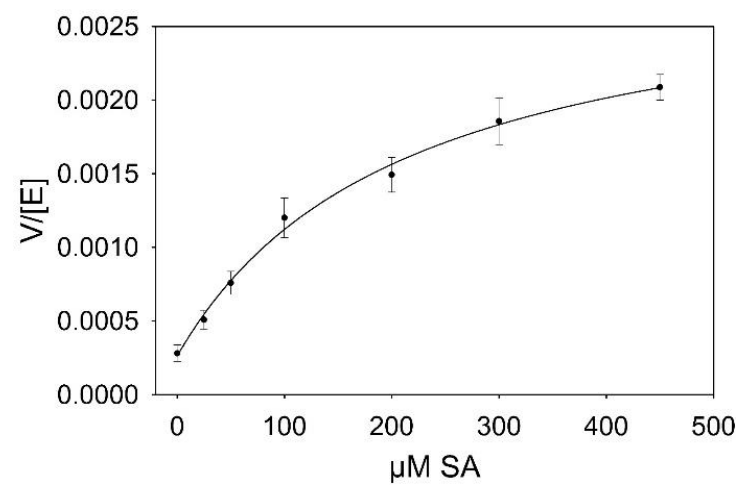

\section{Supplemental figure 15: Enzyme kinetics with 9- and 13-hydroxy-octadecatrienoic acid}

The enzymatic kinetics of UGT76E2, UGT76E11, UGT76E12, and UGT74F1 were determined by spectrophotometric measurements. The UGT-reactions were coupled via the co-product UDP to the pyruvate kinase (PK) and the lactate dehydrogenase (LDH) in $1: 1: 1$ stoichiometry. The PK phosphorylates UDP to UTP while metabolizing phosphoenolpyruvate to pyruvate. LDH catalyzes the reduction of pyruvate to lactate by oxidizing NADH to NAD+, which shows no absorption maximum at $340 \mathrm{~nm}$. The assay was adapted from Brown et al., 2012. The reactions were performed with $5 \mu \mathrm{g}$ UGT76E2 in gel filtration buffer of the respective enzyme, $500 \mu \mathrm{M}$ UDP-Glc, and indicated substrates concentration at $25^{\circ} \mathrm{C}$. The reactions were coupled to $50 \mu \mathrm{g}$ pyruvate kinase, $50 \mu \mathrm{g}$ lactate dehydrogenase, $0.8 \mathrm{mM}$ phosphoenolpyruvate, and $0.15 \mathrm{mM}$ NADH and monitored at $340 \mathrm{~nm}$ for $600 \mathrm{~s}$. A) Kinetics of UGT76E12 with 9-hydroxy-octadecatrienoic acid (9-HOT) and Michaelis-Menten fit of the measurements. B) Kinetics of UGT76E2 with 13-hydroxy-octadecatrienoic acid (13-HOT) and Michaelis-Menten fit of the measurements. C) Kinetics of UGT76E11 with 13-HOT and MichaelisMenten fit of the measurements. D) Kinetics of UGT76E12 with 13-HOT and Michaelis-Menten fit of the measurements. Due to limitations in 13-HOT availability, the value for $150 \mu \mathrm{M}$ was measured only once. E) Kinetics of UGT74F1 with salicylic acid (SA) and Michaelis-Menten fit of the measurements. The data are mean values with standard deviation of three biological replicates. 

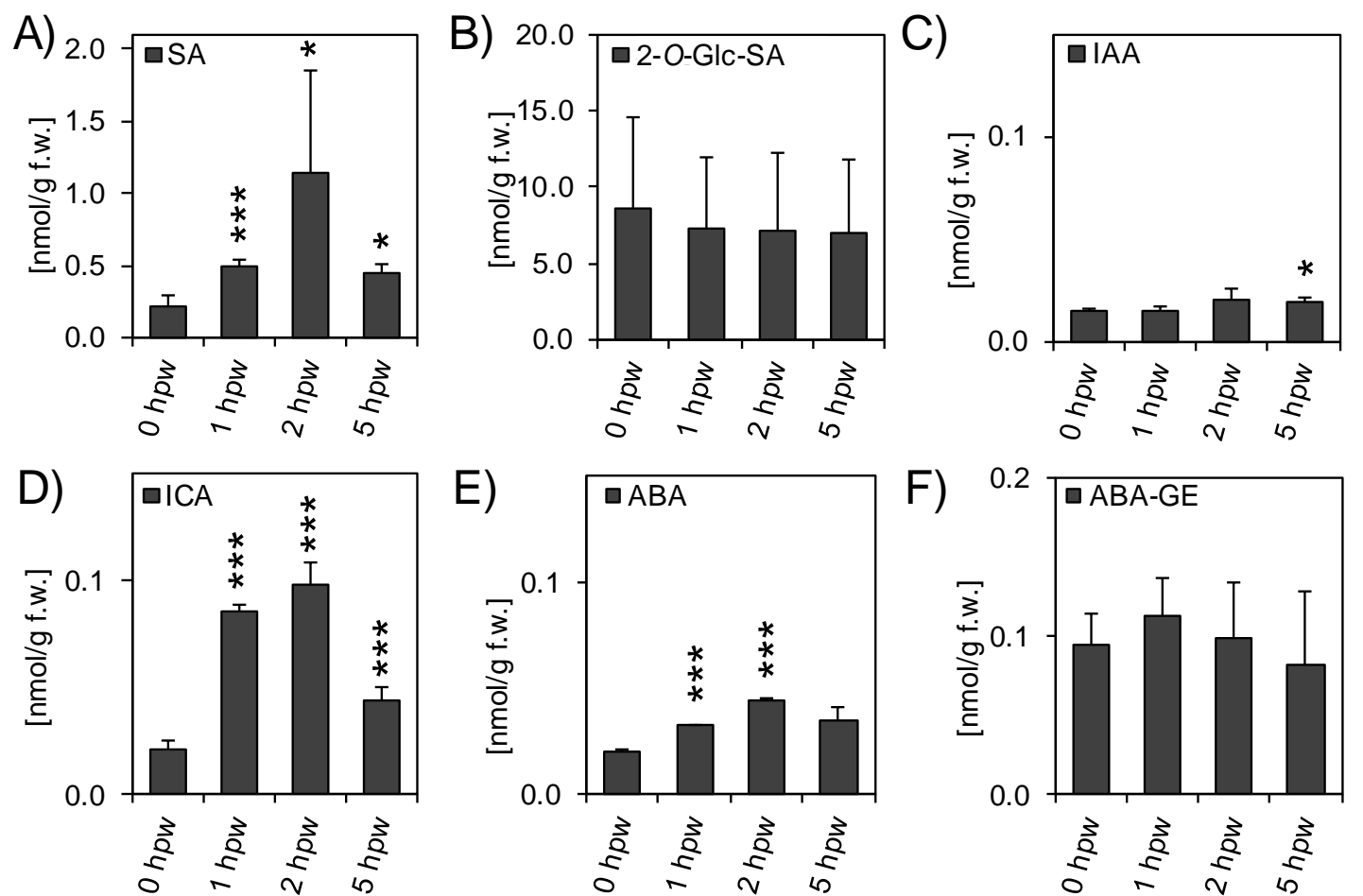

G)

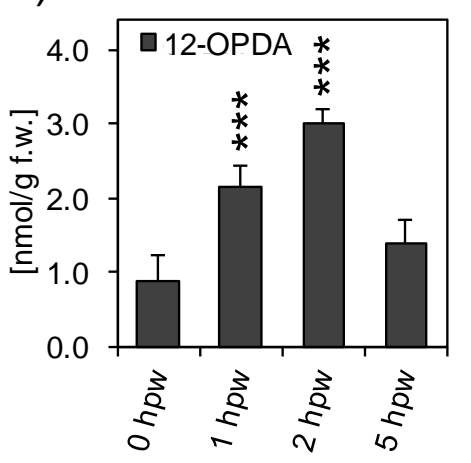

Supplemental figure 16: Phytohormone profiles of A. thaliana after wounding

For the wounding experiment, plants were grown for six weeks at $22{ }^{\circ} \mathrm{C}$ under short day conditions ( $8 \mathrm{~h}$ light/16 h dark). Leaves were wounded three times across the mid vein by squeezing with forceps. Damaged rosette leaves were harvested at indicated time points (hours post wounding (hpw)), extracted, and analyzed by LC-MS/MS. Quantitative data are given in $\mathrm{nmol} / \mathrm{g}$ fresh weight for: A) salicylic acid (SA), B) 2-glucosyl- $O$-SA (2-O-Glc-SA), C) indole-carboxylic acid (ICA), D) abscisic acid (ABA), E) ABA-glucosyl ester (ABA-GE), F) indole-acetic acid (IAA), and G) 12-oxophytodienoic acid (12-OPDA). Each data point represents the mean value + SD of three biological replicates from three independent experiments. 10 plants were pooled for each time point of one replicate. Asterisks indicate significance by one-sided T-Test with *p<0.05, ** $<<.0 .01$, *** $\mathrm{p}<0.005$ Samples were measured by Dr. Cornelia Herrfurth. 


\section{CURRICULUM VITAE}

Sven Haroth

\section{PERSONAL INFORMATION}

Date of birth: $\quad 01.06 .1986$

Place of birth: Alfeld, Germany

Nationality: German

\section{EDUCATION}

Since $01 / 2015$

Doctor rerum naturalium

Department of Plant Biochemistry, Albrecht-von-Haller-Institute for Plant Science, University of Goettingen within the doctorial program "Plant Responses To Eliminate Critical Threats" of the Georg-August University School of Science (GAUSS, Member of the excellence cluster "Göttingen Graduate School for Neurosciences, Biophysics, and Molecular Biosciences”) Thesis title: Metabolic fate of jamonates

\section{$10 / 2012-01 / 2015$ \\ Master of Science}

Department of Plant Biochemistry, Albrecht-von-Haller-Institute for Plant Science, University of Goettingen within the master program „Microbiology and biochemistry"

Thesis title: Catabolic fate of jasmonates

$10 / 2009-09 / 2012$ Bachelor of Science Department of Plant Biochemistry, Albrecht-von-Haller-Institute for Plant Science, University of Goettingen

Thesis title: Generation, purification, and characterization of Lipoxygenase 2 and Lipoxygenase 4 mutants from Arabidopsis thaliana

$07 / 2007-03 / 2007$ Studies of Chemistry, University of Goettingen without degree

$08 / 1993-06 / 2006$ Civilian service at the German Red Cross, Alfeld, Germany General qualification for university entrance Gymnasium Alfeld, Germany Orientation level Alfeld, Germany Primary school „Dohnser Schule Alfeld“, Germany

\section{Publications:}

Bruckhoff, V., Haroth, S., Feussner, K., König, S., Brodhun, F. and Feussner, I. (2016). Functional characterization of CYP94-genes and identification of a novel jasmonate catabolite in flowers. PLoS ONE, 11, e0159875. doi: 10.1371/journal.pone.0159875.

Ding, P., Rekhter, D., Ding, Y., Feussner, K., Busta, L., Haroth, S., Xu, S., Li, X., Jetter, R., Feussner, I. and Zhang, Y. (2016). Characterization of a pipecolic acid biosynthesis pathway required for systemic acquired resistance. The Plant Cell, 28, 2603-2615. doi: 10.1105/tpc.16.00486.

Eng, F., Haroth, S., Feussner, K., Meldau, D., Rekhter, D., Ischebeck, T., Brodhun, F. and Feussner, I. (2016). Optimized jasmonic acid production by Lasiodiplodia theobromae reveals formation of valuable plant secondary metabolites. PLOS ONE, 11, e0167627. doi: 10.1371/journal.pone.0167627. 
